\title{
The Stage-Gate Process, Organizational Politics, and Performance of New Products
}

by

\section{Anju Sethi}

A thesis submitted to the

Faculty of Graduate and Postdoctoral Affairs

in partial fulfillment of the requirements for the degree of

\author{
Doctor of Philosophy \\ in
}

Management

\section{Carleton University \\ Ottawa, Ontario}

(C) 2012

Anju Sethi 
Library and Archives

Canada

Published Heritage

Branch

395 Wellington Street

Ottawa ON K1A ON4

Canada
Bibliothèque et

Archives Canada

Direction du

Patrimoine de l'édition

395 , rue Wellington

Ottawa ON K1A ON4

Canada
Your file Votre référence

ISBN: 978-0-494-93692-4

Our file Notre référence

ISBN: $978-0-494-93692-4$
NOTICE:

The author has granted a nonexclusive license allowing Library and Archives Canada to reproduce, publish, archive, preserve, conserve, communicate to the public by telecommunication or on the Internet, loan, distrbute and sell theses worldwide, for commercial or noncommercial purposes, in microform, paper, electronic and/or any other formats.

The author retains copyright ownership and moral rights in this thesis. Neither the thesis nor substantial extracts from it may be printed or otherwise reproduced without the author's permission.
AVIS:

L'auteur a accordé une licence non exclusive permettant à la Bibliothèque et Archives Canada de reproduire, publier, archiver, sauvegarder, conserver, transmettre au public par télécommunication ou par l'Internet, prêter, distribuer et vendre des thèses partout dans le monde, à des fins commerciales ou autres, sur support microforme, papier, électronique et/ou autres formats.

L'auteur conserve la propriété du droit d'auteur et des droits moraux qui protege cette thèse. $\mathrm{Ni}$ la thèse ni des extraits substantiels de celle-ci ne doivent être imprimés ou autrement reproduits sans son autorisation.
In compliance with the Canadian Privacy Act some supporting forms may have been removed from this thesis.

While these forms may be included in the document page count, their removal does not represent any loss of content from the thesis.
Conformément à la loi canadienne sur la protection de la vie privée, quelques formulaires secondaires ont été enlevés de cette thèse.

Bien que ces formulaires aient inclus dans la pagination, il n'y aura aucun contenu manquant. 


\begin{abstract}
The Stage-Gate process has brought discipline into product development and has led to more efficient allocation of firms' resources. However, an important issue that the StageGate literature has overlooked is the effect of organizational dynamics such as politics and power on the product development process. The literature generally assumes that this process is insulated from organizational politics and power and if gate evaluation is conducted using rigorous criteria then projects will be evaluated solely on their merit rather than on any extraneous considerations including political influence. This dissertation challenges the above assumption and explores if organizational politics and power enter the Stage-Gate process and if this, in turn, impacts the innovativeness of the firm's new products and their performance.
\end{abstract}

Hypotheses were tested with survey data obtained from 120 organizations that use the Stage-Gate process or some variant of it. Results suggest that a political organizational climate is positively associated with accumulation of power by senior managers, and this association is strengthened in the presence of centralization and slack. Power accumulation is positively associated with the politicization of gate reviews. This association does not change in the presence of strict gate review criteria, objective criteria, and an entrepreneurial/innovative culture of the firm. Politicization of gate reviews demotivates product development managers, and this link does not change when the gate review criteria are conditional in nature (i.e., projects can move on to the next stage even if certain criteria have not been met, on the condition that these criteria will be met later). The demotivating experience of product development managers is adversely 
associated with the innovativeness of the new products they develop. This association is worsened in the presence of technological turbulence but improves when there is market turbulence. Finally, innovativeness of a firm's new products is positively associated with market performance.

Further, to better understand the relationships modeled in this dissertation, interviews were conducted with 12 product development managers. These interviews provide good support for the model and explanations for the effects that were not confirmed by the quantitative study. 


\section{ACKNOWLEDGEMENTS}

This dissertation would not have been possible without the guidance and encouragement of many individuals. I am extremely thankful to my advisor, Professor Louise Heslop for her guidance and support through the dissertation process and earlier during my coursework. It has been my good fortune to have had the opportunity to work with a scholar like Louise. I am grateful to her for providing me with the intellectual foundation on which I will be building my future academic career. I would also like to thank my other committee members for their insightful feedback on my dissertation.

I am indebted to several people at Clarkson University, School of Business where I am currently a faculty member. I would like to thank Tim Sugrue, Dean of the Business School for his encouragement and support throughout the Ph.D. process. Also, I am thankful to Larry Compeau, my colleague in the Marketing area, for his wholehearted help during the qualitative phase of the research. Another colleague, Fred Miao helped me with some of the quantitative analysis and I am very thankful to him. I have to express my gratitude to my colleagues John Milne and Marshall Issen for putting me in touch with some of their professional contacts whom I interviewed during the qualitative phase of the research.

I am thankful to Zafar Iqbal and Rajesh Sethi for making their data available for use in this research. I really appreciate their help. 
My deepest thanks go to my family members. I am truly grateful to my husband, Rajesh, for all his support and encouragement during the Ph.D. process. My parents instilled in me the value of education and were very happy when I joined the Ph.D. program. I am thankful for their love, encouragement, and support.

I wish to express my profound gratitude to Mr. Parthasarathi Rajagopalachari, my mentor and guide. His enormous wisdom has benefitted me tremendously. It is with his support and blessings that I was able to successfully complete this endeavour. 


\section{TABLE OF CONTENTS}

ABSTRACT

ACKNOWLEDGEMENTS iv

TABLE OF CONTENTS vi

1. INTRODUCTION 1

1.1 Area of Research and Purpose of the Study 4

$\begin{array}{ll}\text { 1.2 Organization of the Document } & 7\end{array}$

2. LITERATURE REVIEW 9

2.1 The Stage-Gate Process 9

2.1.1 Research on New Product Failures \& $\quad 9$

Successes and Need for the Stage-Gate Process

2.1.2 The Stage-Gate Process and Its Evolution 11

2.1.2.1 The Original Stage-Gate Process 11

2.1.2.2 Revisions in the Original Stage-Gate Process $\quad 15$

2.1.3 The Stage-Gate Process and Radical Innovations $\quad 18$

2.1.4 Further Criticism of the Stage-Gate Process 21

2.1.5 Summarizing the Discussion on the Stage-Gate Process 23

$\begin{array}{ll}\text { 2.2 Organizational Politics } & 25\end{array}$

2.2.1 Different Models of Decision Making 25

$\begin{array}{ll}\text { 2.2.2 Conceptualizing Politics } & 28\end{array}$

2.2.3 Political Behaviour in Organizations $\quad 30$

2.2.4 Politics and Innovation 31

2.2.5 Politics and Its Effect on Employees 35 
2.3.1 Various Conceptualizations of Power Entrepreneurial/Innovative Culture 
4.5 Impact of the Politicization of Gate Reviews on 66

Demotivating Gate Experience

4.6 Moderating Effect of Gate Conditionality 68

4.7 Effect of Demotivating Gate Experience 69 on Innovativeness of Firm's Products

4.8 Moderating Effect of Technology and Market Turbulence $\quad 70$

4.9 Effect of Innovativeness on New Product Performance 72

5. QUANTITATIVE RESEARCH METHOD 75

$\begin{array}{ll}\text { 5.1 Data Collection } & 75\end{array}$

$\begin{array}{ll}5.2 \text { Measures } & 77\end{array}$

$\begin{array}{lr}\text { 5.2.1 Political Organizational Climate } & 78\end{array}$

$\begin{array}{ll}5.2 .2 \text { Slack } & 78\end{array}$

$\begin{array}{ll}\text { 5.2.3 Centralization } & 79\end{array}$

$\begin{array}{ll}\text { 5.2.4 Power Accumulation } & 79\end{array}$

$\begin{array}{ll}\text { 5.2.5 Strict Gate Review Criteria } & 79\end{array}$

5.2.6 Objective Gate Review Criteria $\quad 80$

$\begin{array}{ll}\text { 5.2.7 Entrepreneurial/Innovative Culture } & 80\end{array}$

5.2.8 Politicization of Gate Reviews $\quad 80$

$\begin{array}{ll}\text { 5.2.9 Gate Conditionality } & 81\end{array}$

5.2.10 Demotivating Gate Experience $\quad 81$

$\begin{array}{lr}\text { 5.2.11 Technological Turbulence } & 81\end{array}$

$\begin{array}{lr}\text { 5.2.12 Market Turbulence } & 82\end{array}$

5.2.13 New Product Innovativeness $\quad 82$ 
6. QUANTITATIVE DATA ANALYSIS AND RESULTS

6.1 Item-level Analysis: Missing Values, Outliers, and Normality, 83

$\begin{array}{ll}\text { 6.1.1 Missing Values } & 83\end{array}$

$\begin{array}{ll}\text { 6.1.2 Outliers } & 84\end{array}$

$\begin{array}{ll}\text { 6.1.3 Normality } & 84\end{array}$

$\begin{array}{ll}6.2 \text { Factor Analysis } & 85\end{array}$

6.2.1 Exploratory Factor Analysis $\quad 85$

6.2.2 Confirmatory Factor Analysis $\quad 90$

$\begin{array}{ll}6.3 \text { Reliability and Validity } & 93\end{array}$

6.3.1 Assessing Reliabilities 93

$\begin{array}{ll}\text { 6.3.2 Convergent Validity } & 94\end{array}$

$\begin{array}{ll}\text { 6.3.3 Discriminant Validity } & 95\end{array}$

$\begin{array}{ll}\text { 6.3.4 Nomological Validity } & 98\end{array}$

$\begin{array}{ll}\text { 6.4 Check for Biases } & 98\end{array}$

$\begin{array}{ll}\text { 6.4.1 Non-Response Bias } & 98\end{array}$

6.4.2 Differences between Different Modes of Responding 99

6.4.3 Differences between Respondents from Different 99 Backgrounds

$\begin{array}{ll}\text { 6.5 Hypotheses Testing } & 100\end{array}$

$\begin{array}{ll}\text { 6.5.1 Test of Sub-Model } 1 & 101\end{array}$

6.5.1.1 Inflection Point for the Relationship between 

Demotivating Gate Experience and Innovativeness

6.5.5 Test of Sub-Model 5

6.6 Additional Analysis: Mediation

127

6.6.1 Test of the Mediating Effect of Power Accumulation

6.6.2 Test of the Mediating Effect of Politicization of

130

Gate Reviews

6.6.3 Test of the Mediating Effect of Demotivating Gate Experience

6.6.4 Test of the Mediating Effect of Innovativeness

6.7 Summary of Results

\section{QUALITATIVE RESEARCH METHOD, DATA ANALYSIS, \& RESULTS}

7.1 Qualitative Research Method

139

7.2 Findings of Qualitative Research

7.2.1.1 Effect of Political Organizational Climate on Power Accumulation.

7.2.1.2 Moderating Effect of Centralization on the Political Organizational Climate - Power Accumulation Relationship

7.2.1.3 Moderating Effect of Slack on the Political 
7.2.2.1 Effect of Power Accumulation on

150 Politicization of Gate Reviews

7.2.2.2 Moderating Effect of Strict and Objective

152

Criteria on the Power AccumulationPoliticization of Gate Reviews Relationship

7.2.2.3 Moderating Effect of Entrepreneurial/ 155 Innovative Culture on Power AccumulationPoliticization of Gate Reviews Relationship

7.2.3 Findings for Sub-Model 3

158

7.2.3.1 Effect of Politicization of Gate Reviews on 158 Demotivation of Product Development Managers

7.2.3.2 Moderating Effect of Conditionality on the 160 Politicization of Gate Reviews Demotivation Relationship

7.2.4.1 Effect of Demotivation on Innovativeness 163 of New Products

7.2.4.2 Moderating Effect of Technological Turbulence on the Demotivation Innovativeness Relationship

7.2.4.3 Moderating Effect of Market Turbulence on the Demotivation-Innovativeness Relationship

7.2.5 Findings for Sub-Model 5 and Power Accumulation Low 
7.2.6.2 Powerful Senior Managers and Politicization of Gate Reviews

7.2.6.3 Factors that Keep Politicization of Gate

Reviews Low

7.2.6.4 Differences among Senior Managers and Their Motivations for Power Accumulation

7.2.6.5 Different Perceptions of Technical vs. Non-Technical Product Development Managers Regarding Environmental Turbulence

7.2.6.6 Role of the Project Leader in Influencing Demotivation

7.2.6.7 Role of Individual Level Factors

Technological Turbulence

8.2 Limitations

$\begin{array}{ll}\text { 8.4 Managerial Implications } & 196\end{array}$

$\begin{array}{ll}8.5 \text { Conclusions } & 199\end{array}$ 


\section{List of Tables}

Table 1: Sub-Model-Wise EFA Results $\quad 86$

Table 2: Sub-Model-Wise CFA Results 91

Table 3: Descriptive Statistics of Summary Measures 95

Table 4: Pearson Bi-Variate Correlations $\quad 96$

Table 5: Test of Discriminant Validity 97

Table 6: Regression Results (Sub-Model 1) 101

Table 7: Regression Results (Sub-Model 2) 111

Table 8: Regression Results (Sub-Model 3) 115

Table 9: Regression Results (Sub-Model 4) 118

Table 10: Regression Results (Sub-Model 5) 126

Table 11: Test of Mediation (Mediator - Power Accumulation) 129

Table 12: Test of Mediation (Mediator - Politicization of Gate Reviews) $\quad 131$

Table 13: Test of Mediation (Mediator - Demotivating Gate Experience) 133

Table 14: Test of Mediation (Mediator - Innovativeness) 134 


\section{List of Figures}

Figure 1: Research Framework \& Hypotheses

74

Figure 2: Results of Hypothesis Testing

137 


\section{List of Appendices}

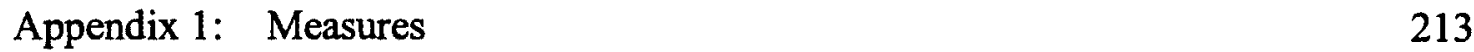

Appendix 2: Item Level Tests for Outliers and Normality 216

Appendix 3: Difference in Responses between Groups 218

Appendix 4: Qualitative Research Interview Protocol 219

Appendix 5a: COMPCO - Interview Summary 234

Appendix 5b: MACHCO - Interview Summary 237

Appendix 5c: FILTCO - Interview Summary $\quad 240$

Appendix 5d: ELECMACO - Interview Summary 243

Appendix 5e: FABRICO - Interview Summary $\quad 246$

Appendix 5f: SOFTCO - Interview Summary 249

Appendix 5g: SEMCO - Interview Summary 252

Appendix 5h: COMAPPCO - Interview Summary 255

Appendix 5i: INDAUTCO - Interview Summary 258

Appendix 5j: ELECTCO - Interview Summary 261

Appendix 5k: APPCO - Interview Summary 264

Appendix 51: HARSOCO - Interview Summary 267

Appendix 6: Summary of Organizations and Respondents in Qualitative 270 Research

Appendix 7: Qualitative Responses for Sub-Model 1: Effect of Political 271 Organizational Climate on Power Accumulation with Moderation by Centralization and Slack

Appendix 8: Qualitative Responses for Sub-Model 2: Effect of Power Accumulation on Politicization of Gate Reviews with Moderation by Strict and Objective Criteria and Entrepreneurial/Innovative Culture 
Appendix 9: Qualitative Responses for Sub-Model 3: Effect of Politicization of Gate Reviews on Demotivation with Moderation by Conditional Criteria

Appendix 10: Qualitative Responses for Sub-Model 4: Effect of Demotivation 274 on Innovativeness with Moderation by Technological and Market Turbulence

Appendix 11: Qualitative Responses for Sub-Model 5: Effect of Innovativeness 275 on Performance 


\section{INTRODUCTION}

New product development is an important activity for firms. Innovative new products can lead to superior performance and sustained competitive advantage (Andrews and Smith 1996; Cooper 2001; Im and Workman 2004). As a result, firms are eager to develop innovative new products. To enhance the possibility of developing innovative new products, companies search for ways to improve the product development process.

One of the systems for improving new product development, which many firms have adopted in the last two decades, is the Stage-Gate process ${ }^{1}$. This process divides the new product development process into a set of stages and activities (e.g. ideation, preliminary investigation, business plan development, product development, testing and validation, full production and market launch stages, Cooper 2001). Each stage is followed by a gate at which projects are evaluated and a decision is made whether the projects should be allowed to proceed to the next stage or not. Senior managers from various departments constitute the Stage-Gate committee which evaluates projects at the gates. Ostensibly, these gates are meant to allow projects that adequately meet evaluation criteria to proceed further and to stop unacceptable projects or allow them to recycle through previous gates (Cooper 2001; Sethi and Iqbal 2008).

While the Stage-Gate process has brought discipline into product development and has led to more efficient allocation of firms' resources, some studies have raised concerns about the process. For instance, it has been argued that the Stage-Gate process may not

\footnotetext{
${ }^{1}$ Stage-Gate is a registered trademark of Product Development Institute Inc.
} 
be suitable for radically new products because radical innovations follow a very different product development process (Veryzer 1998). Some other researchers have found that rigorous implementation of gate review criteria restricts learning by product development teams and this in turn harms the development of novel new products (Sethi and Iqbal 2008). However, an important issue that the Stage-Gate literature has so far ignored is the effect of organizational dynamics or context on the product development process and its outcomes. The Stage-Gate literature generally assumes that this process is insulated from organizational dynamics and the problems these dynamics can create for new products.

However, the literature on organizational politics shows that power and politics play an important role in the innovation process (Frost and Egri 1990a, 1991). According to the organizational politics literature, most organizations are political in nature and political behavior plays a significant role in organizational life. Politics involves efforts by a party to promote its self interest over another (Ferris et al. 1989). An organization's climate will be considered highly political if politics is deeply ingrained in it and political behavior is the main way to get things done (Fairholm 1993). In such a political climate, senior managers can try to acquire power by various means e.g. by gaining control of organizational resources which are a source of power. Power concerns the capacity of individuals to exert their will over others, to create intended effects in line with one's perceived interests (Pettigrew and McNulty 1995), and to overcome resistance offered by other social actors in order to achieve desired objectives (Astley and Sachdeva 1984). 
Political dynamics become particularly salient when new product development is involved. Senior managers want to control new product development because successful new products make the managers controlling these products more powerful in the organization (Frost and Egri 1991). New products may bring additional revenue for the company and thus make the senior manager controlling them more powerful (Pfeffer and Salancik 1978). Also, new products may bring new market and/or operational infrastructure under the control of senior managers who initiated the products, thereby making them more powerful (Hardy and Clegg 1996). At the same time, senior managers don't want others to control successful new products because when others become more powerful, senior managers' own power base is threatened (Frost and Egri 1990a, 1990b). Thus, new product development is an arena where power and politics generally play themselves out.

If politics plays such a significant role in new product development in general, is it really correct to assume that it plays little or no role in the Stage-Gate process? Can the design of the Stage-Gate process insulate the process from organizational politics? For example, the literature seems to suggest that if the Stage-Gate process is well designed and gate evaluation is conducted using rigorous criteria, then projects will be evaluated solely on their merit rather than on any other consideration including political influence. To what extent is the assumption about the ability of criteria rigour to reduce political influence correct? Then, if politics does enter the Stage-Gate process, does it have any effect on the innovativeness of the firm's products and ultimately their performance? These issues 
remain largely unexamined in the Stage-Gate literature. This dissertation addresses this gap.

Related to the above, if politics does enter the Stage-Gate process and influences the outcome of new product development activity, it is important to understand what factors mitigate or enhance the effect of politics. Thus, this is another issue that this dissertation addresses.

\subsection{Area of Research and Purpose of the Study}

The purpose of this dissertation is to examine the existence and effects of organizational politics and power accumulation on the Stage-Gate process and outcomes such as the motivation of product development managers and the innovativeness and performance of new products. It also identifies the factors that mitigate or enhance these effects of politics and power and assesses their influence. The research conceptualizes politics in terms of political climate in the organization.

Specifically, based on the organizational politics and power research (Fairholm 1993; Buchanan and Badham 2003), this dissertation examines if a political climate makes senior managers in the organization strive for more power accumulation. However, a political climate's effect on power accumulation may vary depending on organizational conditions (Bourgeois 1981; Cyert and March 1963; Herold, Jayaraman, and Narayanaswamy 2006; Pfeffer 1981). Therefore, this dissertation also studies if the 
relationship between a political climate and power accumulation is moderated by factors related to organizational structure.

Next, still drawing on power and politics literature, the research examines if powerful senior managers use their power to influence the Stage-Gate process thereby making it political. However, the literature seems to argue that the design of the Stage-Gate process is expected to insulate the process from extraneous influences (Cooper 2001). Research suggests that control in the Stage-Gate process is exercised through the gate review criteria (Cooper 2001; Sethi and Iqbal 2008). Therefore, this dissertation also studies if rigorous gate review criteria can mitigate the effect of power accumulation by senior managers on the politicization of the gate reviews.

The literature also argues that in the case of new and innovative projects, power dynamics are more palpable (Buchanan and Badham 2003; Frost and Egri 1991). Thus, if the organization's culture is more entrepreneurial or innovative, which often produces innovative products (Deshpande, Farley and Webster 1993), it may evoke the use of power by senior managers. Alternatively, such a culture may make it difficult for senior managers to use power and politics to further their own interests (Kanter 1983). Therefore, this research determines if the entrepreneurial/innovative culture of the firm moderates the relationship between power accumulation and the politicization of the gate reviews. 
Further, to understand how politicization of the gate reviews, if it exists, influences the outcome of the product development process, the dissertation draws on the literature that examines the link between politics and employees' experiences and motivation (Drory 1993; Kacmar et al. 1999; Miller, Rutherford and Kolodinsky 2008; Valle and Perrewe 2000). Therefore, it examines if the politicization of the gate reviews leads to a demotivating gate experience for managers who develop new products. However, subsequently the literature has suggested that if the gate criteria are not so rigid, product development managers' experiences may be different (Cooper 2001). Thus, some aspects of the gate criteria that are believed to reduce rigidity in evaluation are examined to determine if they actually do moderate the effect of power accumulation on the motivation of product development managers.

Then, drawing on creativity literature (Amabile 1983), this dissertation studies how the de-motivating experience of product development managers affects the innovativeness of a firm's new products, which is the outcome of interest here. Developing innovative products is challenging and it becomes even more challenging if the firm's environment is turbulent (Brown and Eisenhardt, 1998; Zhou, Yim, and Tse 2005). Therefore, this research examines if turbulence in the firm's environment moderates the effect of managers' experience at gate reviews on the innovativeness of their new products. Finally, this dissertation assesses the relationship between the innovativeness and performance of new products under the conditions of interest to the study. 
This dissertation will make important research and managerial contributions. In terms of research, it challenges the assumption in the Stage-Gate literature that this process is not affected by organizational dynamics such as politics and power, and that the design of the gate reviews can insulate product development from power and politics. Then, by examining how power and politics affect the Stage-Gate process and its outcomes, this dissertation will draw implications about the role of organizational structural factors (e.g., centralization and slack), gate review characteristics (e.g., rigour of the review criteria and their conditionality), and the organizational environmental conditions (e.g., market and technological turbulence) in minimizing or enhancing the influence of power and politics on the development of innovative new products. In terms of practical implications, this dissertation hopes to suggest to top management some ways to achieve more desirable outcomes from the Stage-Gate process (e.g., innovative new products). Similarly, it is expected to inform top management about the conditions in which they need to be extra careful about the harmful effects of politics and power.

\subsection{Organization of the Document}

The remainder of this document is organized as follows. The next chapter reviews three streams of literature that this dissertation draws upon, namely research on the Stage-Gate process, organizational politics, and power. In addition, it focuses on research in related areas including the role of organizational structural and cultural factors and environmental turbulence in influencing power and politics and products developed using the Stage-Gate process. Based on the gaps in the literature, the document then identifies 
and discusses the objectives of this dissertation. Next, the hypotheses concerning the key variables of interest and their relationships that emerge from the literature are presented. The method for quantitative research is discussed after this, and quantitative data analysis and results follow. Then, the research method, data analysis and results are presented for qualitative research. Finally, the findings of the research, its limitations, and research and managerial implications are discussed. 


\section{LITERATURE REVIEW}

\subsection{The Stage-Gate Process}

Currently, most businesses develop new products using some form of the Stage-Gate process (Griffin 1997; Mankin 2004; Schmidt, Sarangee and Montoya-Weiss 2009). A Product Development Management Association's (PDMA) 1995 study reported that nearly 60 percent of firms in the U.S. use some form of the Stage-Gate process (Griffin 1997). In a more recent article, Mankin (2004) stated that "the Stage-Gate process has become the sole method large companies use to bring new products to market" (p.4). Similarly, in an article on new product development project review practices, Schmidt, Sarangee, and Montoya-Weiss (2009) reported that, "the majority of organizations use some variant of a Stage-Gate NPD process" (p.4). The discussion on this process begins in the next section by focusing on the need for the Stage-Gate process.

\subsubsection{Research on New Product Failures and Successes and Need for the Stage-Gate Process}

As customers tastes and needs change, as product life cycles become shorter and existing products quickly become obsolete, and as markets become more global and new competitors enter domestic markets, firms need to constantly innovate and develop new products in order to survive (Cooper 2001; Gorchels 1996; Kahn 2001). New product development (NPD) is also a source of competitive advantage and a means by which 
firms diversify, adapt, and even reinvent themselves to match evolving market and technological conditions (Brown and Eisenhardt 1995).

However, while successful new products are coveted by firms, the development of such products is a challenge. Research has consistently found that failure rates for new products are very high. Cooper (2001) mentioned that most new products never make it to the market. Those that do, face a failure rate in the range of 25 to 45 percent. There are varying estimates of failure rates depending upon the source. The Product Development and Management Association (PDMA) claimed that failure rates were around 41 percent (Barczak et al. 2009). As Crawford (1987) and Cooper (2001) stated, some sources considered the failure rate to be as high as 90 percent, but these claims were generally not supported with data and might be overstated. According to Crawford who undertook a review of these figures, the failure rate seemed to be about 35 percent (Cooper 2001).

These high failure rates have been cause for concern because even with a $35 \%$ failure rate, many companies may not be making the best use of their resources. Prompted by such concerns, several studies were undertaken to understand the factors that lead to new product success and failure. The hope has been that the lessons from these studies could be used to improve the product development process and minimize the failure rate. These studies have a long history, and some of the significant contributors to this stream of research are Crawford (1977), the Conference Board (1980), Cooper $(1979,1980)$, and Cooper and Kleinschmidt (1987) who conducted NewProd studies, and the Product 
Development and Management Association (PDMA) which has conducted a series of best practice studies (2009).

The findings of many of the above studies convinced Cooper that the problems leading to new product failure were detectable and that by controlling or reducing these problems new product success rates could be improved (Cooper 1986). If certain key activities were undertaken and executed well, it would lead to greater new product success. Thus, there was a need for a systematic process to improve new product development, bring discipline, and better allocate or use scarce company resources for new product development. Based on these critical success and failure factors, Cooper felt that firms need to use a formal process for product development and project evaluation. According to Cooper $(1986,2001)$, what was needed was a product development process in which various product development stages are separated by review and evaluation gates leading to the development of the Stage-Gate process.

\subsubsection{The Stage Gate Process and Its Evolution}

\subsubsection{The Original Stage-Gate Process. This process divides the new product} development or innovation process into four or five discrete stages (Cooper 1986). Each stage consists of a set of prescribed, cross-functional and parallel activities and is designed to gather information needed to move the project to the next stage. The entrance to each stage is a Gate. These Gates control the process and serve as the quality control and Go/Kill checkpoints. A typical Stage-Gate process looks as follows: 
Ideation $\rightarrow$ Gate $1 \rightarrow$ Preliminary Investigation $\rightarrow$ Gate $2 \rightarrow$ Build Business Case $\rightarrow$ Gate $3 \rightarrow$ Product Development $\rightarrow$ Gate $4 \rightarrow$ Testing \& Validation $\rightarrow$ Gate $5 \rightarrow$ Full Production and Market Launch $\rightarrow$ Post-launch review.

At the Ideation stage, new product ideas are generated. At Gate 1, these ideas are screened and a Go/Kill decision on the project is made. The project "must meet" and "should meet" certain criteria at this gate. If a Go decision is made, the project moves to Stage 1, which is called Preliminary Investigation.

At this stage, a preliminary market and technical assessment is made. Contact with key users, a concept test and focus groups are done to determine market size, market potential and market acceptance. Also, technical and manufacturing feasibility, possible schedules and costs are explored. Both technical and market information is gathered at low costs and in a short time as input to Gate 2.

At Gate 2, the project undergoes a second and more rigorous screen. It is reevaluated in the light of new information gathered in the Preliminary Investigation stage. At this gate, the project is again subjected to must-meet and should-meet criteria. If the decision is Go at this point, the project moves into a heavier spending phase.

The Business Case stage is the critical homework stage where the detailed business case is built. Market research studies are done to understand customers' needs and wants. A 
detailed competitive, technical, business and financial analysis is done. A product definition is agreed on and a detailed project plan is developed.

An approval at Gate 3 leads to the actual design and development of the new product. If the designed product is approved at Gate 4, the product then goes to the test marketing stage. This is followed by Gate 5. The next stage is the final launch of the product, and thereafter a post launch review is conducted.

The Stage-Gate process is cross-functional in nature (not merely engineering focused) and many functional areas in the company are involved at each stage of the process and at the gates. There is focus on up-front homework or market development work, i.e. market research, competitive analysis, concept tests, manufacturing assessment and business/financial analysis. There is a strong emphasis on market orientation, and so customer needs are determined and market research is done upfront. Also, there is a focus on sharp product definition prior to the beginning of development. Sharp decision points with clear Go/Kill criteria are another feature of the system. Cooper (1994) advocated tough gates with rigorous criteria and metrics - criteria that focus on quantitative, financial measures, as well as qualitative issues such as product advantage and market attractiveness.

Although the original Stage-Gate process had suggested tough gates, Cooper, Edgett and Kleinschmidt (2002b) found that in many companies, gates were quite weak and companies had trouble killing projects. So, they once again suggested implementing 
tough gates, i.e., gates should not just be project review points but tough decision meetings where critical Go/Kill and prioritization decisions are made on projects. Gates must have clear and visible criteria which are effective, i.e. easy to use, realistic, and at the same time discriminating between good and mediocre projects. Gates should have must-meet and should-meet criteria. The review committee should consist of senior managers from functional areas such as marketing, sales, technical, production, and finance representatives. These individuals make the Go/Kill decisions on projects and provide resources needed for the next stage of development (Cooper, Edgett, and Kleinschmidt 2002b).

The important relevant characteristics of gate criteria that have been suggested in the Stage-Gate literature are how strictly criteria are applied and how objective they are (Cooper 1998, 2001). These characteristics are discussed below.

It is suggested that gate review criteria should be formally laid down and strictly adhered to so that there is no confusion and reviewers can do an effective evaluation of projects (Cooper 1994; 2001; Sethi and Iqbal 2008). Also, it is suggested that gates should be tough in order to improve the performance of the development process (Cooper, Edgett, and Kleinschmidt 2002a; 2002b). In addition, criteria should be objective in nature so that they cannot be interpreted differently by different managers and should be consistently applied across all new products (Cooper 1998). Such objectivity prevents gate reviewers from interpreting the criteria in their own way to get their preferred projects through the gates. 
2.1.2.2 Revisions in the Original Stage-Gate Process. Over the years some problems with the original Stage-Gate process were identified. While the Stage-Gate process has introduced more control and order into the product development process of firms, there has also been concern that the process is quite bureaucratic, hard to implement, and time consuming (e.g., Mankin 2004). Cooper (1994) himself recognized some of these problems. For example, projects had to wait at each gate until all tasks had been completed, overlapping of stages was not possible, projects had to go through all gates and all stages, some new product processes were spelled out in far too much detail, and the system did not lead to project prioritization and focus (Cooper 1994).

In order to address these problems, Cooper added some features to the original StageGate process (Cooper 1994, 2001). These features included six F's: Flexibility, Fuzzy or Conditional Gates, Fluidity, Focus, Facilitation, and Forever Green.

Flexibility: The Stage-Gate process can be adapted to the individual project's needs. Some stages can be omitted, and some gates combined. Since Stage-Gate is a risk management process, the risk level, uncertainty, and need for information dictate which stages must be done and which ones can be left out. Typically, lower-risk projects omit some stages and gates (Cooper 2001).

Fuzzy or Conditional gates: This is the primary change that Cooper made to the original Stage-Gate process. Gate decisions no longer have to be either Go or Kill. Instead a Go decision can be made conditional on some future event occurring. Projects move ahead 
and are not held up waiting for one piece of information. If a new product project has not fulfilled all the criteria that had to be met at a particular gate, it does not mean that the project cannot go on to the next stage. It can move on, conditional on the fact that the criteria be fulfilled later and that the results are positive (Cooper 2001).

Fluidity: A project can start the next stage before the previous stage is complete. Long lead time activities can be brought forward from one stage to a previous one, and so stages can be overlapping (Cooper 2001).

Focus: Firms typically try to do too many projects and don't have enough resources to do them well. As a result, there is pipeline gridlock, resources are thinly spread, and good projects are starved for resources. Cooper suggested that the process should be focused and poor projects thrown out at each stage with resources being reallocated to the best projects.

Facilitation: A process manager or facilitator is needed for successful installation of the Stage-Gate process (Cooper 2001).

Forever Green: Stage-Gate processes are constantly being renewed and improved as companies gain experience with them (Cooper 2001).

No doubt, some of the potential problems and shortcomings in Cooper's recommendations in the original Stage-Gate process have been addressed in the revised 
process. For example, the earlier Stage Gate process versions were quite rigid. To rectify this important drawback, Cooper introduced conditional or fuzzy gates. If a project cannot meet certain criteria at a gate, it is not killed or held up; instead it is allowed to move on to the next stage, conditional on the criteria being met at a later stage. This relaxes the rigidity of the gates. Relatedly, the changes in the product development process in the form of the other five F's are also to a large extent an attempt to introduce flexibility into the Stage-Gate process and bring it closer to the reality on the ground in terms of capturing the latest changes in the new product development procedure, such as more simultaneous performance of development activities.

The front end of new product development is known for its fuzziness or lack of clarity. The Ideation stage at the front end is particularly not so well and formally laid out. Cooper makes an attempt to control some of this fuzzy front end by introducing a Discovery stage, instead of the Ideation stage, and a gate that is designed specifically for the Discovery stage. The Discovery stage helps companies proactively capture new product ideas and enhance the flow of good new product ideas into the process. These ideas can come from customers and by understanding their problems, ethnographic research, working with lead customers, or from people within the company (Cooper, Edgett \& Kleinschmidt 2002a).

Cooper (2008) has also discussed different versions of the Stage-Gate process which are suitable for different types of products. He suggests a full Stage-Gate process with five stages and five gates for more risky projects; a Stage-Gate Xpress process with three 
stages and two gates for projects of moderate risk, such as improvements, modifications and line extensions; and a Stage-Gate Lite process with two stages and one gate for very small projects, such as products developed in response to simple customer requests. The higher the risk, the more one follows the full five stage process. Cooper (2008) has also suggested a spiral development process so that project teams can rapidly finalize product design through a number of "build-test-feedback-and revise" iterations (Cooper 2008).

\subsubsection{The Stage-Gate Process and Radical Innovations}

A criticism of the Stage-Gate process has been that while it works for incremental new products, it is not suitable for radical or discontinuous innovations. Radically new products involve "dramatic leaps in terms of customer familiarity and use. ... Frequently these types of products involve the development or application of significant new technologies" (Veryzer1998, p.305). Veryzer has suggested that radical innovations follow a very different product development process. In an in-depth study of eight Fortune 500 firms that consistently produce radical innovations, Veryzer (1998) has shown how these firms use a process that is more exploratory and less customer- driven than the typical incremental new product development project.

Radical or discontinuous projects are generally technology driven, and it may take a long time (up to 10 to 20 years) to develop these products. The process begins because of the convergence of developing technologies, various contextual and environmental factors (such as government regulations), and a product champion or visionary. The 
discontinuous product development process focuses on formulating a product application for the emerging technology. These firms develop prototypes at an earlier stage than the typical incremental project. Prototype production precedes assessment of market attractiveness, market research, and financial analysis activities. Cooper (2001), on the other hand, suggests that detailed market research should be done upfront and only if results are positive should the process proceed further. Prototype development comes much later according to Cooper (2001). Veryzer (1998) suggests that because of the inherent differences in the product development process for radical and incremental innovations, radical innovations should not be assessed using the Stage-Gate process.

A similar criticism of the Stage-Gate process is that it can discourage radical innovation or "really new" products (e.g., Leifer et al. 2000). It has been suggested that really new products require a greater focus on learning rather than on control. Further, even if controls need to be applied on the development of really new products, they cannot be boilerplate controls applied uniformly across all projects. However, the Stage-Gate process does not allow for varying controls for different types of projects (Leifer et al. 2000; McDermott and O'Connor 2002; Veryzer 1998).

A common complaint among researchers and practitioners is that very few radical innovations are launched into the market. Schmidt, Sarangee and Montoya-Weiss's (2009) study substantiates this assertion. Organizations do kill off more radical innovations than incremental ones, and the kill rate increases for radical projects more than incremental projects as the project progresses through the development process. The 
authors found that the reason for this is that organizations use more review points to control radical NPD projects compared to incremental ones, and the number of individuals on the review team is higher for radical innovations. As the number of gates and the number of reviewers increase, it makes the review process more rigorous and demanding, leading to more rejections.

Another study by Ettlie and Elsenbach (2007) explored the nature of the Stage-Gate process used by firms producing radical and incremental innovations. In a study of automotive firms they hypothesized that firms adopting a modified Stage-Gate process are more likely to introduce radical innovations (i.e. high technology products that are new to the world or industry) and that incremental new products would be produced using the standard Stage-Gate process. However, their results did not support this hypothesis. The authors found that firms that produce radical innovations are not more likely to use the modified Stage-Gate process. They did mention that modifications of the process used by respondents most often involve allowing backtracking, but did not give much more detail of what modifications to the Stage-Gate process entail. One reason given for modifying the Stage-Gate process by their respondents was that programme or project management dictates, including continuous improvement guidelines, often overrule Stage-Gate. Ettlie et al. (2007) did find that firms that use the Stage-Gate or modified Stage-Gate process tend to introduce new products more aggressively. They also found that Stage-Gate usage leads to new product success above $50 \%$ levels. Companies adopting a modified Stage-Gate process tend to adopt software that enables teams to work together virtually, and they tend to work in "more formalized 
NPD strategy environments" (Ettlie \& Elsenbach 2007, p.31). They also concluded that with the use of the modified Stage-Gate process, firms can achieve substantial reduction in new product development time without sacrificing product quality.

\subsubsection{Further Criticism of the Stage-Gate Process}

Sethi and Iqbal (2008) highlighted that control of new product development in the StageGate process takes place at gate reviews. Specifically, review criteria were identified as the means to control new product development projects. These authors explored the effect of rigorous gate review criteria (i.e. strict and objective gate review criteria which are frequently applied) on the performance of novel new products (not radical innovations). They defined novel new products on the basis of "the degree to which a product is unique or distinct from competing alternatives or preceding product generations." Taking the position that the Stage-Gate process is not suitable for radical innovations, these authors focused only on non-radical innovations.

Sethi and Iqbal (2008) found that both strict and objective gate review criteria make projects inflexible. Inflexibility refers to the degree to which project parameters become rigid and unchangeable once the project is approved after review at initial gates. These authors also examined if gate conditionality, claimed to be one of the key improvements in the revised Stage-Gate process (Cooper 2001), minimizes the adverse effect of rigorous gate review criteria on project flexibility. However, these authors found that conditionality does not mitigate the effect of rigorous gate criteria on project inflexibility. 
Further, project inflexibility leads to learning failure - i.e. product development teams tend not to acquire new information, incorporate new information into projects, or make changes in plans based on such information (Sethi and Iqbal 2008). This is because when project parameters are fixed, new information cannot be easily incorporated into the project and hence the product development team loses the motivation to learn about the latest developments in the market or technology. Such learning failure, in turn, has an adverse effect on the market performance of novel products. The authors suggested that firms would be better off designing separate gate controls for novel products and even the gate review committee for novel products should be different because these products require a different mind-set during evaluation than incremental products.

Mankin (2004) said that the Stage-Gate process, which is a one size fits all framework, may be reducing firms' new product pipeline. Using examples of several products, he showed how these successful new products that had been launched would never have made it to the market had the Stage-Gate process been used. Instead these products used an iterative process where the product was launched into the market, feedback was garnered, and the product was launched again. The Stage-Gate process would not have allowed this. Mankin (2004) suggested that when the market and the product remain undefined, it is hard to build a compelling justification or a business case for such a product. Thus such products would never get past Gate 2 (or the Business Case stage) and would get killed using the Stage-Gate process. He also indicated that the Stage-Gate process may be more suitable for products targeted at specific, well-defined markets. Instead, he suggested that companies faced with evolving markets or technologies would 
be better served by using an iterative process. In some situations, firms may discover that they lack the best technology to serve their existing or emerging markets. Such technologies may be readily available for purchase at much lower cost than is entailed in internal development.

Chesbrough (2003) introduced the concept of open innovations where new product ideas and technologies can be obtained from anywhere - they don't have to be developed within the firm. The Stage-Gate process with its fast paced orientation to speed up development is not designed to encourage external search. However, an iterative process will lead to such search. In response to this criticism, Cooper (2008) recommended a spiral process that is iterative in nature. He also suggested ways to incorporate open innovation into the Stage-Gate process. Similarly, Gronlund, Sjodin, and Frishammar (2010) have suggested adding the open innovation evaluation criteria to the gate review process.

\subsubsection{Summarizing the Discussion on the Stage-Gate Process}

The Stage-Gate process seems to have brought discipline to new product development, led to more efficient allocation of resources to various projects, reduced costs and cycle

time, and improved new product performance. The design of the Stage-Gate process has been evolving to capture the changes in new product development practice, e.g. the increased usage of cross functional teams, overlapping product development stages, etc. Yet, there is criticism of the Stage-Gate process and its implementation. Some authors 
have suggested that the Stage-Gate process may not be suitable for radical innovations or, at the very least, firms do not fully understand how to use it for radically new products. The process is considered too bureaucratic and rigid.

Control on new product development is exercised at gate reviews through review criteria. The Stage-Gate literature suggests that gate evaluation or gate criteria should be rigorous (i.e., strict and objective) to ensure that new product projects are evaluated on their merit and no other extraneous consideration enters the review process. However, rigorous implementation of the gate evaluation criteria can constrain flexibility and lead to learning failure.

The recent version of the Stage-Gate process, among other things, has tried to relax the rigid nature of the process by making the criteria conditional. The hope is that by allowing conditional approval of projects, team members will not feel constrained and frustrated.

A significant absence from the Stage-Gate literature is the consideration of how organizational issues impact new product development. Particularly, one of the important factors that can seriously impact new product development is organizational politics. The literature overlooks completely how such politics may impact the StageGate process and ultimately the innovativeness and performance of the firm's new products. 


\subsection{Organizational Politics}

Political behaviour plays an important role in organizations. As noted earlier, politics involves efforts by a party to promote its self-interest over another (Fairholm 1993; Ferris et al. 1989). While individuals generally believe that the operations of their organizations involve order, rationality, collaboration and trust, the reality can be different (Buchanan and Badham 2003). While people cooperate, there is also competition; "informal backstaging" takes place along with public action (Buchanan and Badham 2003). Many decisions are taken to preserve and extend the power bases and influence of individuals and groups (Buchanan and Badham 2003).

Major decisions and significant changes are particularly likely to increase political activity. However, decisions are often not defended in terms of political motives and behaviors; instead, reason and logic must be seen as the driver (Buchanan and Badham 2003).

\subsubsection{Different Models of Decision Making}

The political scientist, Graham Allison (1971) made an important contribution to the organizational and government decision making literature by highlighting that three different models can be applied to analyzing important decisions. These are the rational model, the organizational process model, and the political model. He used the example of the Cuban Missile Crisis during the time of the Kennedy administration, to explain 
these models and show how the organizational process model and political model explain reality better than the rational model.

For a long time, organizational decision making has been seen as a rational process in the literature. This model suggests that given the organizational goals and objectives, firms will make a value maximizing, rational choice among alternative courses of action. Allison argued that research draws heavily on rational expectations theories that have their origin in the field of economics. Under such a perspective, actions are analyzed by assuming that nations or organizations consider all possible options and act rationally to select the best option. However, as Allison indicated, it is always possible to come up with an account of value-maximizing choice for any action.

Allison suggested that "rational" analysts need to ignore several facts in order to make their analysis fit their models. In the Cuban missile crisis, for example, rationality would suggest that the threat of mutual destruction due to both the U.S. and the Soviet Union having nuclear weapons would ensure that nuclear war would never occur. However, he showed that while nuclear war did not occur in this instance, it was not because of rational reasons. Nuclear war was quite possible, and contrary to what the rational model indicates, nations could certainly destroy themselves. Allison argued that the rational model did not predict events correctly. Dissatisfaction with the rational model led him to explore the two alternative models of decision making. 
Another way to analyze decisions is by using the "organizational process model." This model, proposed by Allison, is based on March and Simon's (1958) work. Organizations pursue set procedures and existing plans when taking action and generally do not search for new solutions. In the face of time and resource constraints, leaders resort to 'satisficing', i.e., they select the first proposal that sufficiently seems to resolve the problem instead of evaluating all possible courses of action (Simon 1957). Leaders generally prefer solutions that reduce uncertainty in the short term. Allison felt that the process model was an improvement over the rational model, but it still did not capture reality fully.

Therefore, he proposed the political model which according to him was a better way to analyze important decisions. Leaders do not want to acknowledge that politics play a role in their decisions and actions, particularly in critical situations, but in practice politics does influence decisions. According to the political model, decision making and actions are influenced by the process of conflict, power struggles, and consensus building. Self interests of individuals and groups form the bases of the political process (Drory 1993). Leaders' actions are based on politics and negotiations. Leaders differ in terms of how to achieve their goals because they have varying personal interests and backgrounds. They have to gain consensus among their advisors; otherwise their orders can be misunderstood or ignored. This perspective is becoming more widely accepted (e.g. Buchanan 2008; Fairholm 1993; Frost and Hayes 1979). 
The political model suggested by Allison, clearly applies to organizational decision making. Several organization researchers have suggested the political model as an alternative to the traditional rational model, to explain organizational decision making (e.g. Frost and Hayes 1979; Narayanan and Fahey 1982). These researchers suggest that the political model captures organizational reality much better and that organizational decisions reflect a process of power struggle among conflicting individuals and groups, each attempting to further their own self-interest. Contemporary researchers believe that there are limitations to the rationality principle in organizations. These are due to the individual need for power, competition for limited resources, conflicting personal and group interests, etc. The political model takes these limitations into account much better than the other two models discussed by Allison (1971).

\subsubsection{Conceptualizing Politics}

Various definitions of the term "politics" have been offered by different authors. Allen et al. (1979) defined politics as "acts of influence to enhance or protect the self-interest of individuals or groups" (p.77). Mintzberg $(1983,1985)$ defined politics as "individual or group behavior that is informal, ostensibly parochial, typically divisive, and above all, in the technical sense, illegitimate - sanctioned neither by formal authority, accepted ideology, nor certified expertise" (p.172).

Conceptually, some researchers link politics with power and define power "as potential, as the capacity to get others to do things they might otherwise not want to do and/or resist 
others' efforts to get them to do what others want them to do" (Frost and Egri 1990a, p.17). Politics is defined as power in action or "enacted power, as goal directed action that is first of all self-interested and that would be resisted if detected by others with different self-interests" (Frost and Egri 1990a, p.17).

Another conceptualization of politics has been offered by researchers such as Ferris et al. (1989) and Valle and Perrewe (2000). Ferris et al. (1989) defined organizational politics as "a social influence process in which behaviour is strategically designed to maximize short-term or long-term self-interest, which is either consistent with or at the expense of others' interests" (p.145). These authors argued, consistent with earlier theorists (e.g. Gandz and Murray 1980), that it is not actual politics or political behavior that is important but rather the subjective perception of workplace politics, whether actual or not, that generally results in adverse reactions and behaviors. Consistent with Ferris et al.'s definition, organizational politics is generally understood as involving behaviour that is directed toward furthering self or group interest at the expense of others' well-being (Darr and Johns 2004; Kacmar and Baron 1999). Given that politics may not be an objective reality but a subjectively constructed one, most research has measured organizational politics in terms of perceived political activity. This stream of research generally views politics as a part of the organizational climate (Witt et al. 2002).

Politics can operate on an individual level, organizational level, or societal level. Relevant here is the study of politics at the organizational level, given its self- serving nature, in which actions and outcomes concerning a single individual or group have 
consequences for others. Resource interdependence within departments encourages individuals or groups to put their interests ahead of the unit's (Darr and Johns 2004), increasing others' tendencies to engage in such actions or to form perceptions about the presence of such actions in the workplace (Darr and Johns 2004).

Consistent with the work of Ferris et al. (1989) and Witt et al. (2002), in this dissertation politics at the organizational level is viewed as a part of organizational climate. An organization's climate will be considered highly political if politics is deeply ingrained in it and political behaviour is the main way to get things done (Fairholm 1993). Political behaviour refers to efforts by a party to promote its self interest over another (Ferris et al. 1989; Kacmar and Baron 1999).

\subsubsection{Political Behaviour in Organizations}

Studies of perceptions of politics suggest that political behaviour is quite pervasive in organizations. Gandz and Murray (1980) surveyed 400 American managers and found that $90 \%$ of them consider politics to be commonplace, and $89 \%$ said that executives have to be skilled politicians. Drory (1993) argued that political behavior is an entrenched organizational feature. Buchanan (2008) in a study of British managers found that "the experience of politics seems to be frequent, visible and sometimes painful." Only $12 \%$ of the managers surveyed stated that their organization was relatively free of

politics, and only $17 \%$ said that they don't see much political behavior because it happens behind closed doors. 
Furthermore, Buchanan (2008) found that political behavior is not a senior management preserve. Most of the managers surveyed agreed that politics occurs at all organizational levels. As a result, most managers (84\%) agreed that they were ready to play politics when necessary. A vast majority concluded that politics is a natural part of the management job, and that most managers, if they want to succeed, have to play politics at least part of the time (Buchanan 2008).

Markus (1983) identified the triggers of political activity in organizations. These include competition for resources and power bases, and disputes about values, objectives, the nature of the problem and the best solution. Considering these triggers in the context of the conditions in most organizations, political activity would seem the norm, not the exception. The competition of ideas and the combination of personal interest and genuine conviction underlying that competition seems to be quite common. The range of stakeholders in most organizations is quite diverse and most disputes cannot be solved by logic and rational arguments alone. Perceived interests, values and preferences come into play. Decisions are the result of a combination of rational argument supported by lobbying, trading, influencing, coalition building, and other factors (Buchanan and Badham 2003).

\subsubsection{Politics and Innovation}

Frost and Egri (1990a, 1990b) discussed the critical role of politics and power in determining the success or failure of an innovation. Frost and Egri (1991) explained that: 
"Politics is often the inevitable consequence of self-interested contests between and among actors which are engendered by the inherent ambiguity of issues, ideas and things. In that innovation at its core is about ambiguity and is replete with disputes caused by the differences in perspectives among those touched by an innovation and the changes it engenders, we believe that innovation often becomes a political process" ( $p .231)$.

In a similar vein, Kimberly (1980) stated that in modern organizations, "any particular innovation intrudes on a previously negotiated set of agreements about how these interests and priorities are to be accommodated" (p.93).

With the help of several case studies, Frost and Egri (1991) showed that innovations fail or succeed not because they are intrinsically worse or better than existing products but because of politics played by various parties involved. Interests opposed to an innovation use a wide range of tactics to preserve the status quo. In the case of product failures, the innovators turn out to be "less skilful political game players than their opponents." They rely mainly on reason and rational logic to see their innovations through. Opponents of these innovations, on the other hand, use an array of political strategies, leaving nothing to chance. Innovations can be opposed in order to preserve the existing power distribution in the organization or when the interests of powerful individuals are threatened.

Successful innovations involve strategies which are political in nature, e.g., gaining the support of senior management and building networks and coalitions of supporters within 
the organization. Sometimes, in the case of new products, product development work can be done in hiding with bootlegged resources, and later the innovator can ask for forgiveness. However, these managerial actions, also referred to as micro-political strategies, are only effective in the initial stages of product innovation and are not effective once the product leaves the laboratory (Frost and Egri 1991).

Then, if the culture of the organization is innovative, more creative outcomes are likely to emerge in such a set up (Deshpande, Farley, and Webster 1993). Such creative outcomes can evoke political reactions from individuals in a position of power (Frost and Egri 1991). However, it can be suggested that an entrepreneurial/innovative culture may make managers develop more innovative outcomes instead of mainly enhancing their personal and political interests (Kanter 1983).

According to Kelley (1976), politics and innovation are intertwined. Organizations are "arenas where coalitions representing opposing organizational interests meet in conflict." The organizational decision making process reflects this conflict of interests. "Coalitions are usually unstable with each actor getting only part of what he or she wants. If the political risks and costs are greater than the anticipated benefit, the actor will pull out and cut his or her losses. The innovator must constantly guard against withdrawals from a coalition backing the innovation programme; withdrawals can lead to stampedes and the collapse of the project" (Kelley 1976, p.67). When one coalition loses a round in the organizational arena, it does not withdraw from the organization. It bides its time, grows in strength, and fights again. The organizational elites watch clashes between coalitions 
over policy and programmes, and they usually support the winner. They control support which they can withdraw at any time. Thus, the elites are fickle and the innovator "must woo and seduce these fickle elites at critical junctures in the innovation process, to insure the success of the project" (Kelley, p.67).

Smith (2007) explored why innovations are resisted in organizations and how this resistance can be overcome. Innovations lead to change and the novelty associated with innovations often challenges accepted ways of doing things. Work practices can be affected. Moreover changes in technology that are generally part of innovation, often lead to changes in social organization that threaten established hierarchies. Thus innovations can have political consequences and can bring about resistance. This resistance can threaten the project and even lead it to be abandoned. Reluctance to take risks is another factor that can lead managers to favour incremental innovations over radical ones. Further, Smith mentioned how systems and procedures adopted by firms for rational evaluation of new projects can lead to resistance to innovation. These formal screening procedures become obstacles for innovation projects because "they require future benefits to be quantified, but for new products and services this is difficult. Hence these systems can have a bias against innovation, because it can rarely provide the certainty they require" (Smith 2007, p.95). Thus, the innovation process becomes political.

Niccolo Machiavelli, the author of The Prince, has also discussed how difficult it is to get innovations to succeed and why innovations and politics are always interrelated. "And it 
is worth noting that nothing is harder to manage, more risky in the undertaking, or more doubtful of success than to set up as the introducer of a new order. Such an innovator has as enemies all the people who were doing well under the old order and only halfhearted defenders in those who hope to profit from the new. This half-heartedness derives partly from fear of opponents who have the law on their side, and partly from human skepticism, since men don't really believe in anything new till they have solid experience of it" (Machiavelli, 1977, p.17).

\subsubsection{Politics and its Effect on Employees}

A question that arises is: Does politics have an adverse effect on employees and their morale? If indeed the literature suggests that there are consequences of politics for employees, such knowledge can help in examining how politics affects the individuals involved in new product development and thereby the new products they develop.

A great deal of research has been conducted on organizational political climate and its implications for employees' job commitment, job satisfaction, etc. Drory (1993) showed that if employees perceive the organization to be political in nature, their job commitment tends to go down, and so does their satisfaction with supervisors and co-workers.

Studies have typically found an inverse relationship between perceptions of politics and job satisfaction (e.g. Kacmar et al. 1999; Valle and Perrewe 2000). Miller, Rutherford and Kolodinsky (2008) conducted a meta-analysis to examine the relationship between 
employees' perceptions of organizational politics and outcomes like job satisfaction, job stress, turnover intentions, organizational commitment and job performance (also Nye and Witt 1993). Their meta-analysis also found an inverse relationship between perceptions of politics and job satisfaction. Further, they found a significant, positive relationship between perceptions of politics and job stress as well as turnover intentions. Perceptions of politics have also been found to have a negative effect on workers' commitment to the organization.

Another issue of interest for researchers has been the relationship between perceptions of politics and job performance. Researchers suggest a negative relationship between perceptions of organizational politics and job performance (Ferris et al. 2002). Results have shown an inverse relationship (e.g. Vigoda 2000) and a non-significant relationship (e.g. Randall et al. 1999). Miller et al. (2008) in their meta-analysis were also not able to find a clear relationship between perceptions of politics and job performance.

If the organizational climate is highly political, the members of the organization are likely to engage in self-promotional activities (Witt et al. 2002). In such organizations, various interest groups may strive to gain influence and power over others (Galang and Ferris 1997). Generally, when an organization is political, conflict and power struggle are expected to be common (Pfeffer 1981, Pfeffer and Moore 1980).

However, this stream of literature does not say much about the effect of politics on the creativity of employees and therefore, leaves a significant issue unresolved. Yet, as 
discussed earlier, this literature suggests that politics can frustrate and adversely affect job commitment. In turn, according to the creativity literature, such frustration and lack of commitment (i.e. lack of motivation) are known to play an important role in hurting creativity (Amabile 1988). In effect, if not directly, politics seems to have the potential of adversely influencing creativity through employees' motivation.

\subsubsection{Summarizing the Discussion on Politics}

Increasingly, it is being realized that a political perspective to study organizational phenomena may capture reality better than the rational perspective that pervades most of the work on organizations. Researchers have viewed organizational politics as a part of organizational climate.

Politics can play an important role in the innovation process. Innovation at its core is about ambiguity, and this ambiguity can lead to politics. Innovations may succeed or fail not just because they are intrinsically better or worse than existing products, but because politics can play a role. Innovation sets into motion political behaviours in organizations because managers may want to control innovation themselves and may not want others to control it. Thus, if the culture of the organization is more entrepreneurial or innovative and many innovations are developed in different parts of the organization, there is a greater likelihood of politics being set into motion. At the same time, it can be argued that an innovative culture is likely to minimize power and political dynamics (Kanter 1983). 
Politics can have an adverse effect on employees' job satisfaction and organizational commitment, and can increase stress and turnover intentions. Such a climate can also create a struggle for power and influence.

However, the literature on politics and innovation has looked at innovation in general. In present times, a great deal of product development or innovation takes place through the use of mechanisms such as the Stage-Gate process. How a political climate in the organization affects the Stage-Gate process and thereby influences the performance of a firm's new products remains to be studied. It is a particularly important issue to explore because the Stage-Gate literature seems to assume that the design of the process can insulate it from the influence of organizational dynamics like politics (Cooper 2001).

If the organizational climate is political, various interest groups or senior managers may strive to gain influence and power over others (Galang and Ferris 1997). One of the research objectives of this dissertation is to examine if a political climate in the organization spurs the accumulation of power by senior managers and if that is the way through which politics influences the Stage-Gate process. Therefore, it is important to examine how the literature on power can help in the development of the research framework. 


\subsection{Power}

"Power involves the ability of an actor to produce outcomes consonant with his perceived interests" (Pettigrew 1973). According to Hardy and Clegg (1996), power has typically been seen as "the ability to get others to do what you want them to, if necessary against their will, or to get them to do something they otherwise would not." Perrow (1986) defines power as "zero-sum, relational (over someone), exercised both inside and outside the organization, and concerns an output of organized activity that is valued and an output that is produced only at some cost" (p.259).

The sociologist Lukes suggests (1974) "[we] use the vocabulary of power in the context of social relationships to speak of human agents, separately or together, in groups or organizations, through action or inaction, significantly affecting the thoughts or actions of others (specifically in a manner contrary to their interests)" (p.54). Buchanan and Badham (2003) define power as, "the capacity of individuals to exert their will over others" (p.11).

As discussed earlier, politics on the other hand, "is the practical domain of power in action, worked out through the use of techniques of influence and other (more or less extreme) tactics. Thus power is a latent capacity, a resource, or a possession, while politics is power in action" (Buchanan and Badham 2003). 


\subsubsection{Various Conceptualizations of Power}

In one of the earlier conceptualizations, French and Raven (1959) treated power as a property of the relationship between a leader and followers or between an individual and others. Their work provides the foundation for the development of some recent concepts of power that will be used in this dissertation. They identified five different bases of power. These are reward power, coercive power, referent power, legitimate power, and expert power. Reward power is based on the belief that the leader has access to valued rewards which will be dispensed if one complies with instructions. Coercive power is based on the belief that the leader can administer penalties or sanctions that are considered undesirable. Referent power is based on the belief or perception that the leader has charisma, desirable abilities and personality traits that can and should be copied. Legitimate power exists when the leader has the formal authority to give instructions, based on his or her formal position or rank within the organization. Expert power is based on the perception that the leader has superior knowledge relevant to the situation and the task at hand.

These bases of power have two important features. First, they depend on beliefs of others. A leader may be able to dispense rewards and penalties, have superior knowledge, etc., but if others do not believe that the leader has these qualities, then they may be unwilling to comply with requests. On the other hand, leaders may be able to convince others that they have these qualities when in reality they do not. Compliance 
may then be forthcoming. Second, these power bases are interrelated. For example, a leader who resorts to coercive power may lose referent power.

French and Raven's (1959) framework has been extended by Benfari et al. (1986), who identified eight bases of power. To the bases identified by French and Raven, Benfari et al. (1986) added three more. These are information, affiliation, and group power. Information power comes from having access to information that is not public knowledge, because of position or connections. This power can exist at all levels in the organization, not just at the top. Sometimes secretaries of senior executives can have information power and can act as gatekeepers. Affiliation power comes from an authority source with which one is associated. For example, secretaries can act as surrogates for superiors. Finally, group power refers to collective problem solving, conflict resolution, and creative brainstorming. The group can contribute more than an individual.

\subsubsection{A More Recent View of Power}

While the above conceptualizations of power have been quite popular in the literature, many researchers felt that they did not capture the full range of the types of power that are used (Lukes 1974, Hardy 1996). These researchers classified power into three different categories namely resource, process, and meaning power. 
2.3.2.1 Resource Power. Researchers have generally treated power as flowing from the control of scarce resources used to influence behavior in the face of resistance and opposition (e.g. Pettigrew 1972; 1973; Pfeffer and Salancik 1974). According to this conceptualization, "power is exercised by actors to influence decision outcomes and bring about the desired behavior through the use of key resources on which others depend, such as information, expertise, political access, credibility, stature and prestige, access to higher echelon members, the control of money, rewards and sanctions" (Hardy 1996). This form of power works through behaviour modification. As a result, it has limited impact since it is task oriented and the constant use of either rewards or punishment may be necessary to ensure that the desired behavior is sustained. Too much use of coercion may also lead to a reaction in the people over whom power is exercised. As one can see, many of the power bases mentioned by French and Raven and by Benfari et al. are treated as resource power.

Lukes (1974) linked this resource power to early studies of community power. Previously, researchers had studied important decisions that illustrated the power relations existing in a community. The purpose was to determine who made these decisions, i.e., who had the power. If the same groups made the decisions, the community was said to be ruled by an elite. However, the researchers found that different groups prevailed in decision making and they called such communities "pluralist". The assumption behind this work was that power was only used in important decisions where conflict was observable. People were aware of their grievances and tried to redress them by participating in decision making and using power to influence 
outcomes. Given that the decision making arena was open to anyone, if people did not participate in decision-making it meant that they agreed with decisions (Hardy 1996).

However, with time, other researchers began to question and criticize the assumptions underlying the work of the pluralists. This led to the development of the concept of process power.

2.3.2.2 Process Power. Bachrach and Baratz (1962) did not agree that decision making processes were available to everyone, and questioned if non-participation really reflected satisfaction. They said that interests and grievances might not be expressed, and might remain outside the decision- making arena. Conflict could exist even though it was not observable. Hence, full and equal participation could be constrained (Hardy 1996).

Bachrach and Baratz (1962) discuss processes using which "issues are excluded from decision making and the agenda is confined to safe questions" (Hardy 1996). This type of power comes from organizational decision making processes which include a variety of procedures and political routines. These procedures and routines "can be invoked by dominant groups to influence outcomes by preventing subordinates from participating fully in decision making. It has been termed non-decision making, because it allows the more powerful actors to determine outcomes from behind the scenes through the use of procedures and political routines. It highlights the fact that power is not exercised solely in the making of key decisions and that the most visible decision makers are not necessarily the most powerful" (Hardy 1996). Non-decision making is typically used to 
protect the status quo because it tends to support and reinforce existing biases in decision making processes (Hardy 1996).

Bachrach and Baratz (1962) assumed that some conflict was necessary to lead to the use of non-decision making or process power. Lukes (1974), however, felt that conflict did not have to exist for power to be used; instead, he felt that power might be used to prevent conflict from occurring at all. This led to the emergence of the concept of meaning power.

\subsubsection{Meaning Power. Lukes (1974) developed a third aspect of power called meaning} power. He argued that power is often used to shape people's "perceptions, cognitions, and preferences in such a way that they accept their role in the existing order of things, either because they can see or imagine no alternative to it, or because they view it as natural and unchangeable, or because they value it as divinely ordained and beneficial" (p. 24). Thus, dominant groups ensure that threats to their position never arise by invoking the power of meaning whereby individuals accept the status quo because they cannot imagine any alternative. According to this view, the study of power cannot be limited to observable conflict, to the outcomes of decisions or even to suppressed issues. "It must also consider the question of political quiescence" (Hardy and Clegg 1996).

The following discussion focuses on how different aspects of power help or hinder innovation in organizations. 


\section{.2.4 Different Aspects of Power and Innovation}

Dougherty and Hardy (1996) studied sustained product innovation in large, mature organizations. In a study of product innovations in some very large firms, they found that while firms are able to produce a successful new product occasionally, many large organizations have trouble producing sustained product innovation or multiple new products over time. When individual innovation projects were successful in these large organizations, it was mainly because of the efforts of individual managers who used their organizational position and their networks of relationships established over time to gather resources, win senior management support, and promote and protect innovation efforts.

In such organizations, innovation projects typically had trouble getting resources, and innovations were often bumped from agendas and budgets as a matter of course because organizational processes of decision making and budgeting are oriented to routine work,

rather than innovation. Also, innovations had no permanent or inherent strategic meaning in organizations. They found that sustained innovation in large, mature organizations is difficult because "availability of resources, processes, and meaning was piecemeal in most organizations" (p.1146). Dougherty and Hardy (1996) stated that power is needed to facilitate innovation. This power should not be individual but organizational. Product innovation needs to have an explicit, organization-wide power basis; otherwise, there is no energy for developing new products continuously. 
In another study, Hardy and Dougherty (1997) contended that power can both inhibit and encourage innovation. "When organizational power is aligned with innovation, innovators can draw on the organization's competencies to develop viable new products." Hurley and Hult (1998) also suggest that power can help innovation. On the other hand, when "power is aligned against innovation, innovators cannot access the organization's competencies" (Hardy and Dougherty 1997, p.16) and have to protect their ideas from the anti-innovation context. Innovation requires collective action which does not just happen; power is needed to facilitate it.

Product innovation requires "sustained access to organizational resources such as market intelligence, technological expertise," funding, etc. (Hardy and Dougherty 1997, p.17). "However, if the organization's resource power supports routine business, it can work against innovation" (Hardy and Dougherty 1997, p.17). For instance, if resources are controlled by organizational elites who are opposed to new business that does not fit with their skills, innovation may not receive enough funding or other resources. On the other hand, when resource power is aligned with innovation rather than against it, it can foster innovation. For example, if the control of resources such as budgets, information, expertise, political access, etc. is widely distributed in the organization, it is more probable that innovators will get the resources they need. "Ready access to information about customers' needs and trends, operating costs and development expenses" can help product developers (Hardy and Dougherty 1997, p.17). Expertise from other departments can be readily made available to help resolve problems, if innovation is part of people's 
jobs. Managers should be given resources for "pilot production lines, technical problem solving and training" (Hardy and Dougherty 1997, p.17).

Innovation also needs processes to support it. "If the power of processes supports routine business, it can work against innovation" (Hardy and Dougherty 1997, p.18). Senior managers or organizational elites can reserve "the right to decide on all new products behind the scenes, which excludes people who may be more familiar with specific technologies or business opportunities. Decision processes can rely on standards that fit only routine operations (e.g. payback, market share), so innovation proposals never 'measure up' or are 'sent back' for more, perhaps unobtainable, data. Allocation systems such as annual budgeting can be fixed by date rather than opportunity, so new ideas are always put on the back burner" (Hardy and Dougherty 1997, p.18). However, process power can be aligned with innovation if decision criteria and procedures are designed specifically to move innovations through development and to accommodate a regular stream of new products (Hardy and Dougherty 1997).

\subsection{Organizational Structure and Power}

The literature suggests that organizational structure can influence power and political dynamics. This literature is particularly relevant here because it helps in examining one of the research objectives: how organization structure moderates the effect of politics on the Stage-Gate process. 
Among the structural variables that have been more commonly studied by researchers in the areas of power and politics are organizational slack and the centralization of the structure. Slack refers to excess resources that cushion the organization from environmental changes and represent an opportunity for discretionary allocations (Herold, Jayaraman, and Narayanaswamy 2006). One perspective indicates that when there is lack of slack or under conditions of scarcity, there is greater reliance on power to gather resources for one's functional unit (Tjosvold and Poon 1998). Similarly, Gresove and Carroll (1993) argued that absence of slack will trigger attempts by functional units to find ways to influence other units in the organization. In other words, functional units will look for more power - the ability to influence others. On the other hand, it has been argued that when slack is high managers may have a greater tendency to gather resources (Eisenhardt and Bourgeois 1988). In general, slack resources are considered an important requirement for innovation (Herold, Jayaraman, and Narayanaswamy 2006).

Some researchers have suggested that power and political dynamics become more salient when there is a high degree of decentralization (Hage 1980; Pfeffer 1981). However, Eisenhardt and Bourgeois (1988) showed that power and political dynamics are more prevalent when there is centralized decision making. In the case of centralization, these researchers found that there was more competition among executives, which triggers more search for power and the use of politics. In general, according to the innovation literature, centralization hinders innovation (Zaltman, Duncan, and Holbek 1973). 


\subsection{Summarizing the Discussion on Power}

The existing organizational literature views power in terms of resource power, process power, and meaning power. Power can both help and hinder innovation depending on how it is deployed within the firm. Organizational elites or senior managers can acquire resource and process power and use them to prevent innovation that does not enhance their goals and power.

However, whether meaning power is so relevant to the context that is being studied here is questionable. It is a subtle basis of power that is developed over a long period of time. Such power gets gradually institutionalized in the community. For example, the caste system in the Indian society has been used by the members of the upper castes as a subtle means of holding power over the lower castes. It evolved over a long period of time. No one individual can really develop such power in the short or medium term.

In the organizational context, the concept of meaning power looks like a dimension of organizational culture rather than something which a manager can create and accumulate. In fact, it may be quite difficult to show discriminant validity between meaning power and organization culture or mind set. So far, meaning power has mainly been studied in qualitative studies - where researchers can personally probe and identify the presence or absence of such power. Therefore, meaning power does not appear to be a relevant concept for the research framework being developed here. 
Furthermore, Hardy and Clegg's (1996) conceptualization of resource and process power has only been studied in qualitative case studies. Although, on the face of it, these two aspects of power seem distinct, the issue is whether they are really distinct in practice. For example, senior managers who try to accumulate resource power are likely to be equally interested in gaining process power. Thus, in an empirical study, there is always a possibility that these two aspects may not appear as distinct dimensions. As a result, while this dissertation recognizes that power can be gained by controlling both resources and processes, it will not refer to them as two distinct dimensions of power.

Organizational structure influences power dynamics in the organization. Regarding slack, there are two perspectives on its role. One perspective is that an absence of slack can lead to the accumulation of and the exercise of power by various functional units in the organization. The other perspective suggests that high slack may lead functional managers to accumulate more power. Similarly, there are two perspectives on the role of centralization in influencing power accumulation. One perspective is that when the structure is centralized, there will be less accumulation of power. The other view is that if there is centralization, there may be more accumulation of power.

Having looked at the relevant literature on the Stage-Gate process, organizational politics, and power, in the next section, some gaps in these streams of research are identified. 


\subsection{Gaps in the Research}

An important gap in the research that emerges from the above review is how a political organizational climate affects the Stage-Gate process and the performance of new products that are developed using the process.

The literature indicates that a political climate in the organization can lead interest groups to strive for influence or power over others. Does this mean that the effect of organizational politics on the Stage-Gate process is through power accumulation by senior managers in various departments? Therefore, it needs to be understood if organizational politics makes some senior managers acquire more power.

It also emerges from the above review that organizational structural factors such as slack and centralization play a role in influencing power dynamics. However, do slack and centralization influence the extent to which a political climate leads to the accumulation of power in the organization? These issues remain to be examined.

Next, the key issue that is not well understood is, how accumulation of power by senior managers affects new product development through the Stage-Gate process. Do these powerful managers influence and interfere in the Stage-Gate process? As the literature suggests, product development in the Stage-Gate process can be influenced during the reviews of the projects at the gates. Thus, the issue that needs to be studied is, do powerful managers make the gate review process political. 
The above review also indicates that if control is exercised during project reviews through-rigorous criteria, new product projects will be evaluated and given permission for development based solely on their merit without any extraneous influence. The literature suggests that rigorous criteria refer to strict and objective gate review criteria. Strict and objective gate review criteria can prevent or minimize the influence of power. Does this mean that the effect of the accumulation of resource and process power on politicization of the gate reviews is likely to be mitigated by the rigour of the review criteria?

The above review also suggests that an entrepreneurial/innovative culture has a role in influencing the degree to which politics is set into motion. Does this mean that an entrepreneurial/innovative culture is likely to moderate the relationship between power accumulation and the politicization of gate reviews? This moderating effect of entrepreneurial/innovative culture remains to be explored.

Further, what is the effect of politicization of gate reviews? The literature review suggests that politics can have a frustrating or demotivating effect on individuals in the organization. Does this finding also apply to the effect of the politicization of gate reviews? Therefore, it is important to examine the effect of the perception of politics during gate reviews on the degree of demotivation of product development managers who negotiate their products through the Stage-Gate process.

What is the consequence of the demotivating experience of product development managers? Will product development managers' demotivation affect their ability to 
develop superior or innovative new products? As such, the effect of demotivating experience on the innovativeness of new products needs to be studied.

It is normally challenging to develop innovative new products (Cooper 1996). Will the challenge be greater if the firm is operating in turbulent environments? The literature has focused on two components of environmental volatility, market and environmental turbulence (Jaworski and Kohli 1993). Thus, it is important to examine if these two types of environments moderate the effect of demotivating experience on the innovativeness of new products.

Finally, there is this old research question, does innovativeness matter? In other words, does it have any impact on performance? As such, the effect of the innovativeness of new products on their performance needs to be studied.

This dissertation aims to address these gaps in the literature. In the next chapter, research objective are listed. 


\section{RESEARCH OBJECTIVES}

\subsection{Specific Research Objectives}

The objectives of the research are to determine:

1. the extent to which a political organizational climate leads to the accumulation of power by senior managers affected by new product development.

2. the degree to which organizational structural factors (slack and centralization) moderate the effect of a political organizational climate on power accumulation by senior managers affected by new product development.

3. to what extent power accumulation by senior managers leads to the politicization of gate reviews.

4. the degree to which rigorous gate review criteria (i.e., strict and objective criteria) moderate the effect of power accumulation on politicization of gate reviews.

5. the extent to which the entrepreneurial/innovative culture of the organization moderates the effect of power accumulation on politicization of gate reviews. 
6. the degree to which politicization has a demotivating effect on the product development managers who negotiate their products through the Stage-Gate process.

7. the extent to which the conditional nature of the review criteria moderates the relationship between politicization of gate reviews and demotivating experience of product development managers.

8. the degree to which product development managers' demotivation has an adverse effect on the innovativeness of new products.

9. the extent to which environmental factors (i.e., market and technological turbulence) moderate the relationship between demotivated managers and the innovativeness of new products.

10. the degree to which the innovativeness of new products affects the performance of these products. 


\section{HYPOTHESES DEVELOPMENT}

\subsection{Political Organizational Climate and Power Accumulation}

A political organizational climate is one in which politics is deeply ingrained in the organization and the main way to get things done is through political behavior (Fairholm 1993). Senior managers in such organizations understand that they should be in a position to do political maneuvering in order to get things done (Fairholm 1993; Buchanan and Badham 2003). For this, they need power or the ability to influence. As Pfeffer (1981) states "to get things done, you need power- more power than those whose opposition you must overcome" (p.46). Thus, when an organization's climate is political, senior managers try to acquire power or influence for their own effectiveness (Buchanan and Badham 2003; Pfeffer 1992).

Furthermore, highly political climates are infused with conflict (Darr and Johns 2004). In such climates, strong interdepartmental conflicts often surface. Conflict increases the use of influence strategies (Welsh and Slusher 1986). When there is conflict in organizations, there is a tendency to engage in activities that increase power (Jehn 1997). In effect, when the organizational climate is political and there is conflict, senior managers try to increase their power.

Controlling resources and processes in the organization is the way through which senior managers can accumulate power. Senior managers who control key resources become 
powerful (Hardy 1994; Hardy and Clegg 1996). When a senior manager possesses resources that are critical but relatively scarce, others who need and value such resources become dependent on the senior manager. The manager may share these resources with others but in return expects favours and support for his/her agenda. Others know that if they don't support the senior manager's agenda, critical resources can be withheld from them.

Similarly, power resides in organizational decision making processes which incorporate a variety of procedures and routines that can be invoked by senior managers to influence outcomes (Bachrach and Baratz 1970; Hardy 1994; Hardy and Clegg 1996). Senior managers who have some control over decision making processes can set these up in a way that suits them and helps them attain their objectives, while denying others the opportunity to have their way (Bachrach and Baratz 1970; Hardy 1994; Hardy and Clegg 1996). These managers can also control the agenda for meetings and discussions, so that only the issues or projects they favour come up for discussion and the ones they do not favour have a reduced possibility of being discussed - at least in the short or medium run. Others in the organization may want to support these powerful senior managers' preferred projects so that when the time comes, their own projects receive a favourable nod from these managers for consideration and approval (Bachrach and Baratz 1970, Hardy and Clegg 1996).

In sum, in political organizational climates, senior managers tend to accumulate power by controlling resources and processes. Therefore, 
H1: The more political an organization's climate, the more its managers accumulate power.

However, the tendency to accumulate power may not be the same across different types of organizations (Cyert and March 1963; Moch and Pondy 1977). In other words, in a political organizational climate, power accumulation by senior managers may differ depending upon certain organizational conditions. For instance, the literature suggests that two types of organizational factors can influence the power dynamics in organizations. The first factor is slack, which is defined as a surplus of resources available to the firm after resources have been allocated to meet immediate business requirements, fund ongoing projects, or meet explicit objectives (Cyert and March 1963). In other words, slack represents excess resources that cushion the organization from environmental changes and make discretionary allocations possible, such as for innovation activities (Bourgeois 1981; Herold, Jayaraman and Narayanaswamy 2006).

The other factor is the centralization of the organization structure (Cyert and March 1963; Pfeffer 1981; Eisenhardt and Bourgeois 1988). A highly centralized organization is one in which there is more concentration of decision making authority in the hands of a few top managers (Zaltman, Duncan, and Holbek 1973; Jaworski and Kohli 1993; Menon et al. 1999). However, the question is, do slack and centralization of the organization structure moderate the relationship between political organizational climate and power accumulation? 


\subsection{The Moderating Effect of Slack and Centralization}

4.2.1 Organizational Slack. One perspective on the role of slack suggests that in a political organizational climate, when slack is high, managers will have a greater tendency to accumulate resources (Eisenhardt and Bourgeois 1988). Since a political organizational climate is rife with conflict, senior managers have a strong tendency to acquire influence. Thus, when resources are available and are "up for grabs," it is an opportunity for senior managers to accumulate them and consolidate their influence (Eisenhardt and Bourgeois 1988). However, under conditions of organizational conflict, senior managers who have accumulated slack resources cannot let others control the decision making processes and agenda and see their influence diminished. Thus, in a political organizational climate, managers who have accumulated slack resources will also try to acquire process power to wrest as much control as possible.

The other perspective suggests the opposite role of slack (Cyert and March 1963; Moch and Pondy 1977). In a political organizational climate, even though there may be conflict, in the presence of a lot of slack resources, senior managers' tendency to accumulate resources as a way to acquire power may not be high. In a plentiful slack environment, since resources are widely available to everyone, controlling some resources may not give these senior managers any real influence (Cyert and March 1963; Tjosvold and Poon 1998). Even if this contention is true, organizational conflict, along with a desire for control will make senior managers search for some other source of influence. Thus, these managers may shift their focus to acquiring process power. In 
effect, in the presence of high slack, even if resources do not bring any real power, senior managers will tend to control processes - the only remaining source of influence. Therefore,

H2: The higher the slack in an organization, the stronger the positive effect of a political organizational climate on senior managers' power accumulation.

4.2.2 Centralization. In a political organizational climate, if the organizational structure is centralized, some authors argue that there will be less accumulation of power (Pfeffer 1981). Since in a centralized organization, decision making authority is in the hands of the top management team, these scholars expect conflict to be subdued. However, this dissertation focuses on power accumulation at the level of senior managers who are usually a level below the top management team. Generally, such senior managers have to get things done in the organization and for that reason they need influence and power in a political climate.

Some other researchers argue that when decision making is concentrated at the very top in the organization (say with the CEO), the desire to control and influence on the part of senior managers is enhanced (Eisenhardt and Bourgeois 1988). According to these researchers, considering the political climate in the organization, senior managers have a desire for control and to play a role in organizational decision making and feel frustrated by their autocratic chief executives. Thus, they try to find ways to accumulate power or the ability to influence. In other words, in a political climate, whenever there is 
centralization of decision making, senior managers will have a greater tendency to gain influence (Eisenhardt and Bourgeois 1988). Therefore,

H3: If the organizational structure is centralized, the effect of a political organizational climate on power accumulation will be enhanced.

\subsection{Power Accumulation and Its Influence on Gate Reviews}

Innovations can be game changers. They can make the managers controlling these innovations powerful. Thus, senior managers use innovation to preserve and enhance their power (Buchanan and Badham 2003; Frost and Egri 1991). Powerful managers would, therefore, like to influence innovation.

At the same time, innovations or new products can threaten those who are currently powerful in the organization. As Machiavelli said, "The innovator makes enemies of all those who prospered under the old order..." $(1977$, p. 17). Senior managers who were powerful and dominant under the "old order" and who stand to lose their power if an innovation is implemented would like to obstruct or thwart the innovation. Such powerful senior managers will use all their power to frustrate new ideas or innovations, unless these are under their own control. As Frost and Egri (1991) also argue, if a proposed innovation threatens existing power interests, it evokes the full array of opposing political forces. 
In the context of Stage-Gates, it is the gate review committee which decides the fate of a new product development project (Cooper 2001). The committee can approve a project or reject it or ask for more work on the project before the project can proceed further to the next stage of development. Thus, if new products or innovations are to be influenced, the way to influence them is through the gate review committee. Powerful senior managers would like to influence action in the gate review committee to maintain their hold on power.

The gate review committee is usually made up of representatives of different functional areas within the organization (Cooper 2001). Powerful senior managers exert influence either themselves, if they serve on the committee, or through their representatives on the committee. They may also attempt to influence heads of other functional areas and through them their representatives on the gate review committee. In return for support, senior managers with resources under their control can offer to share resources and those who control the process and agenda can promise favorable decision outcomes. These promises do not have to be explicit; heads of other functional areas understand that by supporting these powerful managers they are earning credits that they can encash in the future. Jointly, senior managers' own representatives on the gate review committee and other representatives who have been brought on board will support senior managers' favourite projects and oppose the ones they don't favour. However, the other functional heads and their supporters whose projects are being opposed will not give in so easily. They will try to keep the projects alive in an attempt to retain or enhance their own power. Thus, gate reviews are likely to become an arena for turf battles between 
functional areas. As a result of the above maneuverings, the managers who shepherd their new products through the Stage-Gate process will find the gate reviews highly politicized. Thus,

H4: The more power senior managers accumulate in an organization, the higher the politicization of gate reviews.

Can the effect of power accumulation on politicization of gate reviews somehow be influenced? One set of factors that can affect the above relationship is related to the design of gate reviews. It is argued in the literature that gate reviews and evaluation criteria are set up in such a way that they do not allow any extraneous influences to enter into the evaluation process. By implication, the evaluation criteria are not likely to allow even organizational power and political dynamics to seep into the review process. The Stage-Gate literature suggests that the criteria need to be rigorous, i.e. they should be strict and objective (Cooper 2001; Sethi and Iqbal 2008). Strict criteria mean that criteria are formally laid out and strictly followed. Objective criteria refer to the fact that the criteria are not open to interpretation or negotiation and are uniformly applied to all projects (Cooper 2001; Sethi and Iqbal 2008). Objective criteria do not vary based on who is doing the reviewing and so they do not vary from project to project. It is not known, however, if strict and objective criteria can mitigate the effect of power accumulation by senior managers on the politicization of gate reviews. 
The other factor that can affect the relationship between power accumulation and the politicization of gate reviews is the entrepreneuria/innovative culture of the firm. An entrepreneurial/innovative culture is one which emphasizes creativity, entrepreneurship, and risk taking (Deshpande, Farley, and Webster 1993). However, it has not been studied if such a culture can moderate the effect of power accumulation on politicization of the Gate review process.

\subsection{Moderating Effect of Strict and Objective Gate Review Criteria and Entrepreneurial/Innovative Culture}

4.4.1 Strict Criteria. Although power accumulation by senior managers and its use in the gate reviews may politicize the reviews, if the gate evaluation criteria are strictly implemented, it may help reduce the politicization of gate reviews. If the criteria are formally laid out and strictly applied across all projects, managers responsible for product development clearly understand in advance the criteria against which their new product will be evaluated. These managers are likely to ensure that the new product development project meets all the criteria before it is brought before the review committee. If a project meets all the criteria, it may become difficult to create obstacles to its approval (Cooper 2001). Therefore, even if powerful senior managers do not favour a project for political reasons, they may find it harder to get it rejected by the review committee. In effect, strictness of the criteria reduces the senior managers' degrees of freedom to politically manipulate the review process. Therefore, 
H5: The stricter the gate review criteria, the weaker the effect of power accumulation by senior managers on the politicization of gate reviews.

4.4.2 Objective Criteria. Even though the gate review criteria are strict, still there is the possibility that the review committee may interpret the criteria leniently to suit their favourite projects and harshly to create obstacles for the projects they don't favour. However, gate evaluation criteria that are objective, are not open to subjective interpretation or negotiation and do not vary with the reviewer (Cooper 2001). For example, at the end of a concept test, when the project is brought to the gate, the review criteria can state that a high level of customer acceptance is necessary for the project to be approved. Such a criterion is open to interpretation. On the other hand, if the criterion states that at least sixty percent of customers must respond that they are likely or highly likely to buy the product, for the project to be approved, such a criterion is more objective. Objective criteria are likely to restrict committee members' freedom to manipulate the interpretation of criteria to favour certain projects over others. Thus, even though some powerful managers would like to politically influence the review process, their ability to do so is reduced due to the criteria's objectivity. Therefore,

H6: The more objective the gate review criteria, the weaker the effect of power accumulation by senior managers on the politicization of gate reviews.

4.4.3 Entrepreneurial/Innovative Culture: An entrepreneurial/innovative culture can keep power and political dynamics low in the organization. Such a culture may drive 
managers to do what will enable the organization to produce more innovative outcomes instead of mainly promoting their personal and political interests (Kanter 1983). For example, as the literature suggests, in firms such as $3 \mathrm{M}$ and Intel, an innovative culture tends to keep everyone including powerful senior managers focused on the development of innovative new products (O'Reilly 1989) and these managers tend to rely less on other considerations in supporting new products.

However, it is possible to argue that increased innovation is likely to spur more political reactions (Frost and Egri 1990, 1991). Yet, given the argument that an entrepreneurial/innovative culture is expected to foster attitudes, behavioral expectations, and practices that promote innovation and discourage actions that inhibit innovation (Bartel and Garud 2009), this dissertation argues that powerful senior managers are less likely to politically interfere when the organization is more focused on innovation. Therefore,

H7: The more entrepreneurial/innovative the organizational culture, the weaker the effect of power accumulation by senior managers on the politicization of gate reviews.

\subsection{Impact of the Politicization of Gate Reviews on Demotivating Gate Experience}

If managers who take their new products through the gate reviews believe that the gate reviews have become highly politicized, their motivation is likely to be adversely affected. Research has shown that when employees see politics operating in the 
organization, their job satisfaction goes down (Miller, Rutherford and Kolodinsky 2008) and so does their job commitment (Drory 1993). Politics also leads to disillusionment, frustration, and stress (Ferris et al. 1994; Kacmar et al 1999; Valle and Perrewe 2000; Miller et al 2008). Similarly, when gate reviews becomes politicized, product development managers who take their products through the gate review process are likely to feel demotivated. There is generally an expectation of fairness and rationality in the review process. Most managers expect that if they worked hard and came up with a superior product that is good for the organization, it should be supported and encouraged by senior managers. However, when they see that their superior product receives little support at gate reviews and it is not the quality of the project, but politics that determines which projects are supported, they are likely to find it unfair and feel frustrated and demotivated. Therefore,

H8: Politicization of gate reviews leads to a demotivating experience for product development managers.

Will politicization of gate reviews have the same adverse effect on new product development managers under all circumstances? There has been an attempt to make gate reviews less rigid by making gates conditional. Conditionality means that if a project cannot meet certain criteria at a gate, it is not killed or held up; instead it is allowed to move on to the next stage, on the condition that the criteria are met at a later stage (Cooper 2001; Sethi and Iqbal 2008). Is it possible that gate conditionality may 
help alter the effect of the politicization of gate reviews on product development managers' motivation?

\subsection{Moderating Effect of Gate Conditionality}

If gate reviews get politicized, the review committee can argue that a project they do not favour does not adequately meet the gate criteria, or the committee can contend that there is not sufficient proof that the project can achieve the desired level of market, technical or financial performance. Such a project would likely be delayed or killed. However, if the gates are conditional, it is possible that these criteria are likely to be allowed to be met at a later stage (Cooper 1994, 2001). Similarly, if gates are conditional, product development managers can request that they be allowed to provide the required proof of market, technical or financial feasibility at a later gate. Thus, by cleverly using conditionality, product development managers can blunt or neutralize the adverse effect of such politically motivated objections. Since politics is not immediately hurting their project, product development managers may feel less frustrated or demotivated. Therefore,

H9: Gate conditionality will reduce the adverse effect of the politicization of gate reviews on the demotivating experience of product development managers. 


\subsection{Effect of Demotivating Gate Experience on Innovativeness of Firm's Products}

Motivation plays a critical role in the generation of innovative outcomes (Andrews and Smith 1996; Kanter 1983, 1988). The social psychology literature considers motivation the primary ingredient for innovativeness (Amabile 1983, 1988). In the case of new products, innovativeness requires discovering novel linkages among customer needs and technology and the company's expertise. Such discovery involves experimentation and trial and error. This process of discovery can lead to many dead-ends and may require repeatedly exploring new avenues, which can be a frustrating experience (Hogarth 1980; Osborn 1963). Therefore, it is essential that product development managers should have a high degree of motivation. Without such motivation there can be a tendency to give up and not persevere until success in developing an innovative product is achieved.

Furthermore, this discovery process is risky because the possibility of failing to achieve an innovative outcome is high (Hogarth 1980; Osborn 1963). With concern for possible failure comes the fear of social ridicule and the loss of prestige. In such circumstances, motivation plays a key role in producing a creative outcome. Lack of high motivation can keep one from pursuing the potentially risky path of discovery. When managers feel demotivated because of the experience they have at the gates, it will lead them to produce less innovative new products. As a result, the innovativeness of the firm's new products will suffer. Thus, 
H10: A demotivating experience at the gates will harm the innovativeness of a firm's new products.

Developing new products is particularly challenging in turbulent environments.

Environmental turbulence refers to the rate and uncertainty of changes in the organization's environment (Dess and Beard 1984, Miller and Friesen 1983). The literature has suggested two types of environmental turbulence - technological and market (Kohli and Jaworski 1990). Technological turbulence refers to rapid changes in technology (Jaworski and Kohli 1993). Market turbulence refers to rapid changes in the tastes and needs of customers and the speed at which competitors enter and leave the market (Jaworski and Kohli 1993). However, it remains to be examined if technological and market turbulence moderate the adverse effect of a demotivating gate experience on the innovativeness of firm's products.

\subsection{Moderating Effect of Technological and Market Turbulence}

In a turbulent environment, developing innovative new products requires far more motivation on the part of product development managers. When markets are turbulent, customer needs change rapidly and present a moving target, and competitors move in and out of markets and frequently change their strategies. Similarly, when there is turbulence in the technological environment, technologies evolve rapidly and often in unexpected ways. In such turbulent environments, developing innovative products is particularly challenging (Danneels and Sethi 2011). For example, what might be 
considered innovative for the existing customer needs, competitive offerings, and state of technology, might not remain so when these sectors of the environment undergo a major change due to turbulence. Product development managers may start development with some assumptions about the market and technologies, but may have to make major changes or even totally abandon their initial approach as the environment changes drastically (Danneels and Sethi 2011).

In other words, there is a possibility that a lot of effort put into developing the innovative product may go to waste. Similarly, due to turbulence, it may be difficult to predict the market and technology scenario that will prevail in the future, because several different scenarios may seem equally plausible. Thus, product development mangers may have to simultaneously work on several alternative products for different potential scenarios (Danneels and Sethi 2011). In the end, it is possible that only one among several such products may be considered relevant and innovative and the rest may have to be abandoned. Such conditions can be very challenging and frustrating for product development managers. To effectively deal with such circumstances, product development managers need to have a very high degree of motivation. If managers lack strong motivation, they may find it particularly difficult to develop innovative new products under conditions of technological and market turbulence. Thus,

H11: Market turbulence will worsen the adverse effect of a demotivating gate experience on the innovativeness of a firm's new products. 
H12: Technological turbulence will worsen the adverse effect of a demotivating gate experience on the innovativeness of a firm's new products.

\subsection{Effect of Innovativeness on New Product Performance}

Innovativeness is known to play a crucial role in the success of new products. In one of the earlier attempts in this area, Crawford (1977) reviewed a number of studies and concluded that a lack of innovativeness is the single most important reason for new product failure. Subsequently, many other studies have argued for the positive effect of innovativeness on performance (Cooper 2001; Sethi, Smith and Park 2001). New products that satisfy customer needs in an innovative manner and thus win over a loyal customer following, generally lead to higher sales and market share (Cooper 2001;

Crawford 1977). Sometimes such products also offer an opportunity to charge a higher price and thereby enjoy a higher profit margin. Furthermore, innovativeness generally gives a long-term competitive edge to new products. Thus, the sales, market share, and profitability gains from such products can last a long time. Therefore,

H13: The higher the innovativeness, the better the performance of new products.

The hypothesized relationships are included in a research framework in Figure 1. The hypotheses concerning the nature of the relationships among the research framework concepts have been developed only for those relationships that are of interest in this research and are supported by the research cited. Indeed, some other relationships of a 
direct or indirect nature and possibly feedback relationships may exist among the concepts in the model. However, the research proposed here is an initial investigation in the area of organizational politics and the role of the exercise of power in the Stage-Gate NPD review processes. Therefore, it was necessary to propose a reasonably coherent and limited set and form of relationships to study. Not all possible relationships could be studied without losing focus and clarity in the research. The relationships posited in the framework are believed to be important driving factors and supportable by research. Therefore, they are the focus of this dissertation. 
Figure 1

Research Framework and Hypotheses

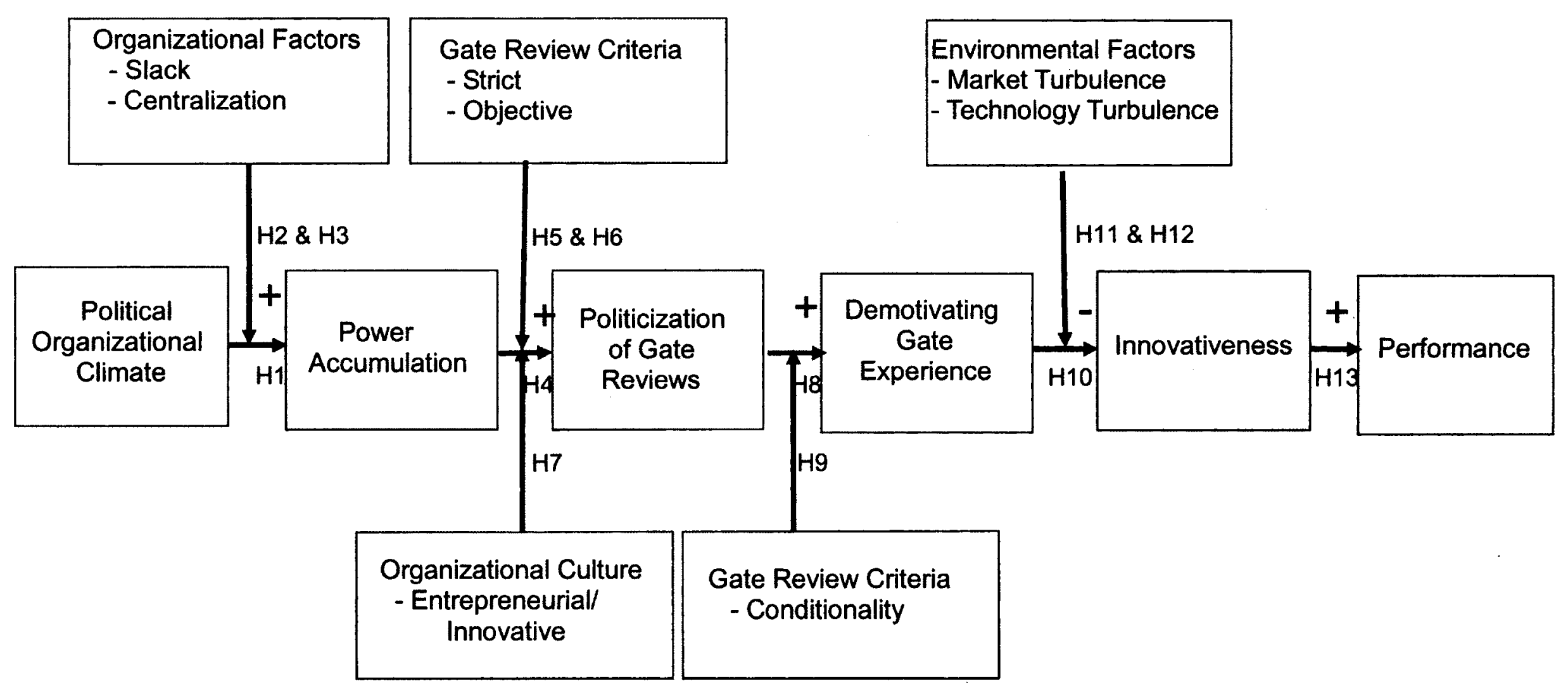




\section{QUANTITATIVE RESEARCH METHOD}

The research method used to test the research framework has two components - one quantitative and the other qualitative. First, the quantitative method used for testing the hypotheses is presented in this chapter and the analysis is discussed in the following chapter.

\subsection{Data Collection}

Hypotheses were tested using an existing data set based on a survey of new product development managers that deals with the issues included in this dissertation ${ }^{2}$. The data includes information on organizational issues such as organizational power and politics, organizational environment, organization structure, the firm's Stage-Gate process, managers' experience through the Stage-Gate process, and the performance of the firm's new products. Since the dissertation focuses on organizational level issues, this data was drawn on to test the hypothesized model.

The survey data was collected from key informants. In the current context, this person would be the manager who has been responsible for new product development projects and thus would be senior enough to be adequately knowledgeable about the organization and its processes for new product development.

\footnotetext{
${ }^{2}$ Professors Zafar Iqbal (DePaul University) and Rajesh Sethi (Clarkson University) provided this data. They have used part of the data to study project level issues about gate reviews in the Stage-Gate process. The unused organizational level data was used for this dissertation. This dissertation is an independent inquiry and was not a part of the research plan of the data collectors.
} 
The criteria for participation in the survey were that: (1) the organization to which the responding manager belonged should use the Stage-Gate process for new product development, (2) the responding manager should have had the experience of taking projects through the gate reviews (3) the manager surveyed should have completed a new product development project within two years preceding the survey as a project manager so as to be familiar with the details of the gate review process (Sethi and Iqbal 2008). To identify key informants, secondary sources of information, such as a variety of tradepublications were searched to create a list of firms that had developed and introduced new products within two years prior to the survey. The firms that satisfied the above criterion were contacted to identify project managers who met the criteria for participation. These managers were approached and requested to participate in the study. The managers who agreed were e-mailed a questionnaire to complete. Managers had the choice of returning their completed surveys either through fax or mail (Sethi and Iqbal 2008).

Questionnaires were e-mailed to 200 project managers. These managers worked in medium to large enterprises. In some cases, their organizations were newly formed divisions or business units in medium to large enterprises. A reminder with another copy of the questionnaire was e-mailed to managers who had not responded after two weeks. Responses were received during a 2-month period following the original e-mail. Usable responses were received from 120 managers, which is a $60 \%$ response rate for completion of the questionnaire among those to whom it was sent. This is a high response rate for such studies, suggesting the relevance of the topic to these managers 
and the benefit of prior contact with respondents (Erdogan and Baker 2002; Harzing 2000; Stafford 1966; Van Kenhove, Wijnen, and De Wulf 2002). The industries in the sample mainly consisted of telecommunications, light engineering products, chemical products, consumer software, food, and home appliances.

The average business unit in the sample had 11,090 employees $($ median $=9050$, range $=$ 55 to 100,000 ), sales of $\$ 4.1$ billion (median $=4.0$, range $=.02$ to 45 ), and had been in existence for 29 years (median $=21$, range $=3$ to 125 ). Business units in the sample were typically medium to large firms and some were newly formed units of medium and large enterprises. On average, the Stage-Gate process had been in place for 5 years. The executives that took the survey had an average new product development experience of 7 years. The respondents reported that they were quite knowledgeable about various aspects of the company and the Stage-Gate process. The average knowledge on a sevenpoint scale was 5.8 ( 7 being highly knowledgeable). The respondents came from diverse functional areas: marketing (38\%), product development and R\&D (31\%), general management (15\%), and manufacturing (16\%).

\subsection{Measures}

The unit of analysis for this dissertation is an organization. Therefore, all the concepts are assessed at the organization level. For example, organizational politics and power accumulation by various senior managers are organizational level concepts.

Politicization of the Stage-Gate process is again across projects and thus is more akin to 
an organizational level concept. Similarly, demotivating experience during the StageGate process is being conceptualized across projects. Gate review criteria are also organizational level concepts.

To the extent possible, existing measures of various concepts are being used. Appendix 1 contains the measurement items for each concept of interest.

5.2.1 Political Organizational Climate. This new measure assesses the extent to which politics is ingrained in the organization and if work gets done mainly through political behavior (Fairholm 1993). The approach to assessing this concept is consistent with the politics literature (Fairholm 1993; Ferris, Russ, and Fand 1989; Witt, Kacmar, Carlson, and Zivnuska 2002). The seven items for political organizational climate were measured using a seven-point strongly agree - strongly disagree response format. An example of an item for this measure is, "Engaging in political behavior is the way to get things done in our business unit."

5.2.2 Slack. This measure captures the availability of slack in the firm, including human and financial resources. An existing measure, developed by Danneels and Sethi (2011) has been used for slack ( $\alpha=.71$ for the original measure). The measure for slack has four items and uses a seven-point strongly agree - strongly disagree response format. One of the items for this measure is, "Our business unit has a reasonable amount of resources in reserve." 
5.2.3 Centralization. This measure captures the concentration of decision making authority in the hands of a few top managers (Zaltman, Duncan, and Holbek 1973; Jaworski and Kohli 1993; Menon et al. 1999). An existing measure, developed by Jaworski and Kohli (1993), was used to assess the level of centralization in the organization ( $\alpha=.88$ for the original measure). Centralization was assessed with a set of eight items, using a seven-point strongly agree - strongly disagree response format. An example of an item is, "There can be little action taken here until a senior manager approves a decision."

5.2.4 Power Accumulation. This new measure captures accumulation of both resource and process power. The items in this measure are consistent with the literature on power (Hardy 1994; Hardy and Clegg 1996). The measure for power accumulation has seven items and uses a seven-point strongly agree - strongly disagree response format. One of the items for this measure is, "Decision criteria for important issues are controlled by only a few senior managers."

5.2.5 Strict Gate Review Criteria. The measure for this concept evaluates the degree to which the gate review criteria are formally laid down and used and strictly enforced. This is an existing measure developed by Sethi and Iqbal (2008) $(\alpha=.89$ for the original measure). The measure for strict criteria has three items and uses a seven-point strongly agree - strongly disagree response format. An example of an item for this measure is, "The criteria for gate reviews are strictly adhered to." 
5.2.6 Objective Gate Review Criteria. The measure for Objective Gate Review Criteria refers to the extent to which projects are evaluated using criteria that cannot be subjectively interpreted or negotiated for each project. This is also an existing measure (Sethi and Iqbal 2008) ( $\alpha=.74$ for the original measure). One of the items for this measure is, "The criteria for gate reviews are open to negotiations (reverse-coded)." The measure for objective criteria has seven items and uses a seven-point strongly agreestrongly disagree response format.

5.2.7 Entrepreneurial/Innovative Culture. This measure assesses whether the culture of the organization encourages creativity or innovativeness, risk taking, and entrepreneurship. To determine whether the organization has an entrepreneurial or innovative culture, an existing measure developed by Deshpande, Farley and Webster (1993) was used ( $\alpha=.66$ for the original measure). This measure has three items and uses a seven-point strongly agree - strongly disagree response format. An example of an item for this measure is, "The glue that holds my business unit together is a commitment to innovation and development. There is an emphasis on being first."

5.2.8 Politicization of Gate Reviews. This new measure captures whether or not the gates are politicized and are grounds for turf battles. The measure is consistent with the politics literature (Buchanan and Badham 2003; Fairholm 1993). This measure has four items and uses a seven-point strongly agree - strongly disagree response format. One of the items for this measure is, "Gate reviews are grounds for turf battles for functional managers." 
5.2.9 Gate Conditionality. This measure assesses the extent to which projects are allowed to move on to the next stage even if they have not met all the criteria at a gate, subject to the condition that the criteria are met at future gates. This is an existing measure (Sethi and Iqbal 2008) ( $\alpha=.67$ for the original measure). This measure has three items and uses a seven-point strongly agree - strongly disagree response format to determine the extent of conditionality in the gate reviews. One of the items for this measure is, "Gate reviews of projects allow a project to be approved even if all criteria are not met, conditional on those criteria being met in the future."

5.2.10 Demotivating Gate Experience. This measure captures the extent to which the gate experience was dissatisfying and motivation reducing. The items in this new measure are consistent with the literature on politics and motivation (Kacmar et al. 1999; Valle and Perrewe 2000). This measure has three items and uses a seven-point strongly agree - strongly disagree response format. An example of an item for this measure is, "The experience of gate reviews is satisfying" (reverse-coded).

5.2.11 Technological Turbulence. This measure assesses the degree to which rapid changes in technology and breakthroughs occurred in the environment creating opportunities for new product development. It is based on Jaworski and Kohli's (1993) measure of technological turbulence ( $\alpha=.88$ for the original measure). This measure has four items and uses a seven-point strongly agree - strongly disagree response format. One of the items for this measure is, "The technology in our industry is changing rapidly." 
5.2.12 Market Turbulence. The measure for market turbulence captures the degree of volatility in customer needs and competitors' actions. This is an existing measure (Jaworski and Kohli 1993) ( $\alpha=.68$ for the original measure). This measure has four items and uses a seven-point strongly agree - strongly disagree response format. An example of an item for this measure is, "It is difficult to predict how customers' needs and requirements will evolve in our markets."

5.2.13 New Product Innovativeness. This new measure assesses the extent to which the firm introduced innovative and breakthrough products. It is consistent with an existing innovativeness measure (Im and Workman 2004; Sarin and McDermott 2003). The New Product Innovativeness measure has four items and uses a seven-point strongly agree strongly disagree response format. One of the items for this measure is, "Introduce breakthrough products that have taken our business unit in newer directions."

\subsubsection{New Products' Performance. The new products' performance measure is} consistent with an existing measure (Sethi 2000) ( $\alpha=.92$ for the original measure). The measure for performance has three items and uses a seven-point not at all - a great deal response format. The key informants were asked to rate the new products' performance in terms of sales, market share, and profits. 


\section{QUANTITATIVE DATA ANALYSIS AND RESULTS}

Data analysis was started by screening the data for missing values, outliers, and normality at the item level. Then, factor analysis was done to assess the measures. Both exploratory and confirmatory factor analyses were conducted. Details of these analyses are given below.

\subsection{Item-level Analysis: Missing Values, Outliers, and Normality}

6.1.1 Missing Values. Only $0.5 \%$ of the values in this sample were missing. Out of total 54 items, there were 34 items (63\%) with no missing values, 12 items (22\%) with one missing value, six items (11\%) with two missing values, and two items (4\%) with three missing values. None of the cases had more than 4 missing values. Out of 120 cases, 100 cases ( $83 \%)$ had no missing values, 14 cases $(12 \%)$ had only one missing value, two cases (2\%) had two missing values, three cases (3\%) had three missing values, and only one had four missing values.

When the proportion of missing values is small, the literature suggests that mean substitution is as effective as other techniques of dealing with missing values (Cheung 2007; Rubin et al. 2007). Since in the current sample the proportion of missing values was very small, mean substitution was used for replacing missing values, i.e. the mean of all other individuals' responses on a particular item was used to replace missing values for that item. 
6.1.2 Outliers. In order to identify outliers in the data, the method suggested by Hair et al. (2006) was used. These authors recommend that observations for each variable should first be converted to standard scores or $\mathrm{z}$ scores. For samples with more than 80 observations, cases with standard scores of 4 or greater should be treated as outliers (Hair et al. 2006). This method was followed and $\mathrm{z}$ scores for all observations were obtained. The $\mathrm{z}$ values for the data ranged from -3.29 to 3.35 with one exception. For the variable power accumulation 4 a minimum $\mathrm{z}$ value of -4.27 was obtained, indicating one outlier (see Appendix 2). However, since the $\mathrm{z}$ value for this outlier was close to the cutoff, it was decided to first examine if this variable passed the test of normality. The item power accumulation4 reads, "Certain senior managers in my business unit have significant control on strategic and operational decisions."

6.1.3 Normality. The data was next examined for normality. According to the literature, univariate skewness and kurtosis above 2 for an item are considered high (Muthen and Kaplan 1985; Gao, Mokhtarian and Johnston 2008). In the data, skewness and kurtosis range from -1.26 to 1.5 , with the exception of the item power accumulation 4 which has a kurtosis value of 2.84 (see Appendix 2). This suggests that the item power accumulation 4 cannot be considered normal. Given that this item was not normal and had an outlier in it, it was decided to drop this item from further analysis.

Having screened the data for missing values and replacing them, checking for outliers, and ensuring normality, the data was ready for further analysis. Before the items could be aggregated to obtain concept-level scores, it was important to examine if various items 
loaded on their respective concepts. Therefore, exploratory and confirmatory factor analyses were conducted as discussed below.

\subsection{Factor Analysis}

6.2.1 Exploratory Factor Analysis. First, measures were refined using exploratory factor analysis (EFA) with Principal Axis Factoring (PAF) and Oblique rotation was used.

Oblique rotation was preferred because it accommodates correlated factors. A minimum cut off of eigenvalue 1 was used for extracting factors (Hair et al. 2006). A Scree Plot was obtained to determine how many factors should be retained. Items with loadings of +0.40 on a factor are minimally acceptable (Hair et al. 2006).

Four different EFAs were run, one for each sub-model in the framework, with all the concepts in the sub-model included. The performance concept was excluded from this analysis because it is a formative measure (Diamantopoulos and Winklhofer 2001). Thus, EFA was not run on the fifth sub-model that captures the innovativenessperformance relationship.

First, EFA was conducted on all the concepts, including the moderators, used to examine the effect of a political organizational climate on power accumulation (i.e. political organizational climate, slack, centralization and power accumulation). This is referred to as sub-model 1. Six factors with eigenvalues above 1 emerged. However, the scree-plot indicated the existence of four factors. Thus, four factors were retained for this sub- 
model. All factor loadings were above 0.4 except one (see Table 1). The one exception was PowAccu2 with a loading of 0.20 and so this item was dropped from further analysis. All other items loaded on their respective concepts and cross-loadings were minimal (below 0.4) and so all these items were retained.

Table 1

Sub-Model-wise EFA Results (Principal Axis Factoring with Oblique Rotation)

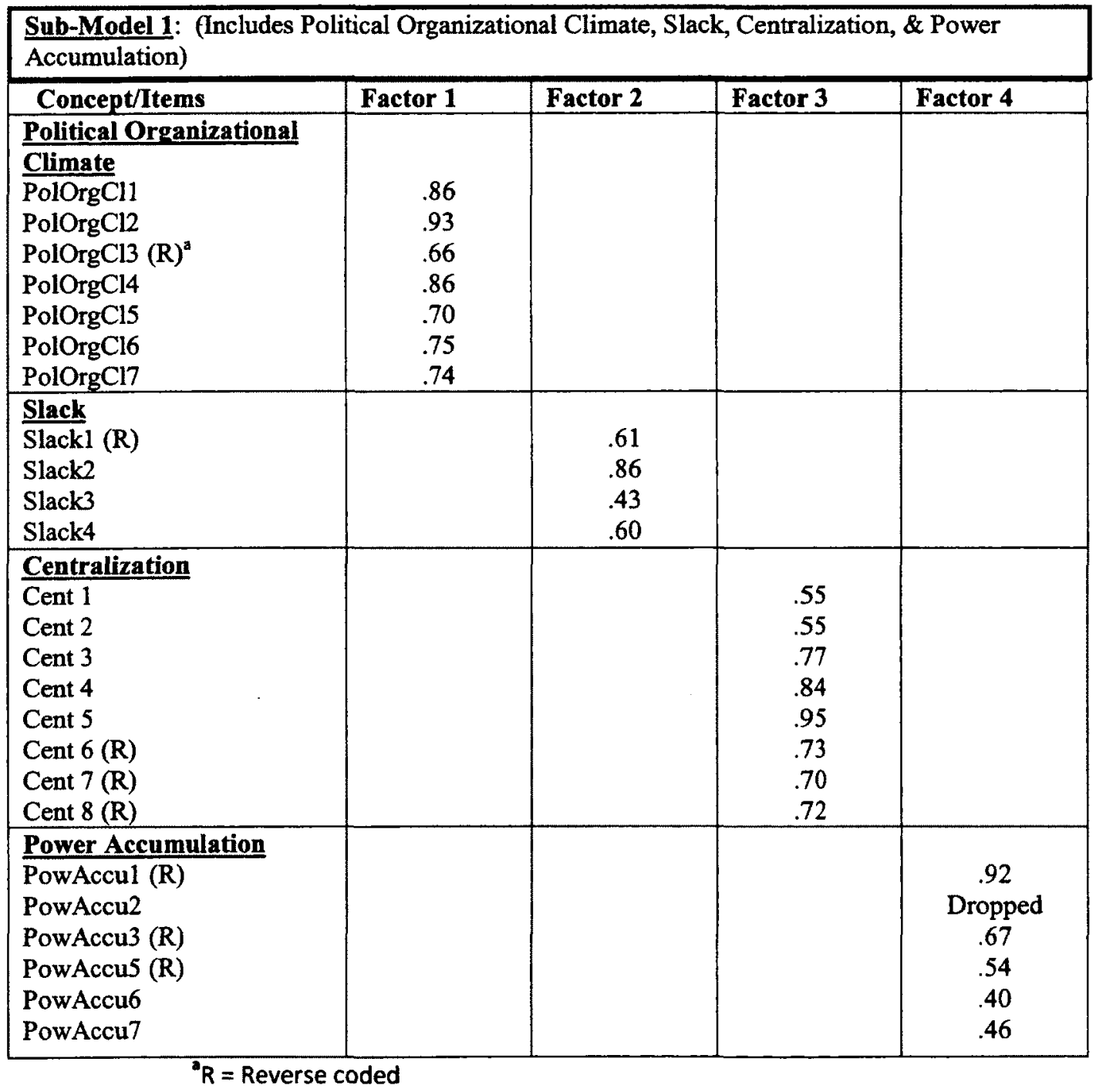




\begin{tabular}{|c|c|c|c|c|c|}
\hline \multicolumn{6}{|c|}{$\begin{array}{l}\text { Sub-Model 2: (Includes Power Accumulation, Strict Criteria, Objective Criteria, } \\
\text { Entrepreneurial/Innovative Culture, \& Politicization of Gate Reviews) }\end{array}$} \\
\hline & Factor 1 & Factor 2 & Factor 3 & Factor 4 & Factor 5 \\
\hline $\begin{array}{l}\text { Power Accumulation } \\
\text { PowAccu1 (R) } \\
\text { PowAccu3 (R) } \\
\text { PowAccu5 (R) } \\
\text { PowAccu6 } \\
\text { PowAccu7 }\end{array}$ & $\begin{array}{l}.90 \\
.72 \\
.51 \\
.51 \\
.52 \\
\end{array}$ & & & & \\
\hline $\begin{array}{l}\text { Strict Criteria } \\
\text { StrictCr1 } \\
\text { StrictCr2 } \\
\text { StrictCr3 }\end{array}$ & & $\begin{array}{l}.90 \\
.95 \\
.68 \\
\end{array}$ & & & \\
\hline $\begin{array}{l}\text { Obiective Criteria } \\
\text { ObjCr1 (R) } \\
\text { ObjCr2 (R) } \\
\text { ObjCr3 (R) }\end{array}$ & & & $\begin{array}{l}.81 \\
.56 \\
.61\end{array}$ & & \\
\hline $\begin{array}{l}\text { Entrepreneurial } \\
\text { Innovative Culture } \\
\text { InnovCul1 } \\
\text { InnovCul2 } \\
\text { InnovCul3 }\end{array}$ & & & & $\begin{array}{l}.67 \\
.68 \\
.74 \\
\end{array}$ & \\
\hline $\begin{array}{l}\text { Politicization of Gate } \\
\text { Reviews } \\
\text { PolGateRev1 } \\
\text { PolGateRev2 } \\
\text { PolGateRev3 (R) } \\
\text { PolGateRev4 } \\
\end{array}$ & & & & & $\begin{array}{c}.78 \\
.68 \\
.54 \\
\text { Deleted } \\
\end{array}$ \\
\hline \multicolumn{6}{|c|}{$\begin{array}{l}\text { Sub-Model 3: (Includes Politicization of Gate Reviews, Gate Conditionality, Demotivating Gate } \\
\text { Experience }\end{array}$} \\
\hline Concept/Items & Fac & & Factor 2 & & ctor 3 \\
\hline $\begin{array}{l}\text { Politicization of Gate } \\
\text { Reviews } \\
\text { PolGateRev1 } \\
\text { PolGateRev2 } \\
\text { PolGateRev3 (R) }\end{array}$ & & & & & \\
\hline $\begin{array}{l}\text { Gate Conditionality } \\
\text { CondCr1 } \\
\text { CondCr } 2 \\
\text { CondCr } 3\end{array}$ & & & $\begin{array}{l}.49 \\
.89 \\
.57\end{array}$ & & \\
\hline $\begin{array}{l}\text { Demotivating Gate } \\
\text { Experience } \\
\text { Demotexp1 } \\
\text { Demotexp2 (R) } \\
\text { Demotexp3 (R) }\end{array}$ & & & & & $\begin{array}{l}59 \\
.75 \\
69\end{array}$ \\
\hline
\end{tabular}




\begin{tabular}{|l|c|c|c|c|}
\hline \multicolumn{5}{|l|}{ Sub-Model 4: (Includes Demotivating Gate Experience, Market Turbulence, Technological Turbulence, \& } \\
\hline Innovativeness) & Factor 1 & Factor 2 & Factor 3 & Factor 4 \\
\hline Demotivating Gate & & & & \\
\hline Experience & .54 & & & \\
Demotexp1 & .80 & & & \\
Demotexp2 (R) & .66 & & & \\
Demotexp3 (R) & & .80 & & \\
\hline Market Turbulence & & .77 & & \\
MktTurb1 & & .84 & & \\
MktTurb2 & & .50 & & \\
MktTurb3 & & & .78 & \\
MktTurb4 & & & .75 & \\
\hline Technological & & & .78 & \\
Turbulence & & & .76 \\
TechTurb1 & & & & \\
TechTurb2 & & & & \\
TechTurb3 & & & & \\
TechTurb4 (R) & & & & \\
\hline Innovativeness & & & & \\
Innov1 & & & & \\
Innov2 & & & & \\
Innov3 & & & & \\
Innov4 & & & & \\
\hline
\end{tabular}

Then, an EFA was run on sub-model 2. This sub-model consisted of all the concepts, including the moderators, used to examine the effect of power accumulation on politicization of the gate reviews (i.e. power accumulation, strict gate review criteria, objective criteria, entrepreneurial/innovative culture, and politicization of the gate reviews). Since the item PowAccu2 was dropped after the EFA for sub-model 1, this item was not included in the EFA for sub-model 2. Five factors with eigenvalues above 1 emerged. The scree-plot also confirmed the existence of five factors. All factor loadings except one were above 0.4 (see Table 1). The one exception was PolGateRev4 with a loading of 0.22 and so this item was dropped from further analysis. All other items 
loaded on their respective concepts and cross-loadings were minimal (below 0.4) and so all these items were retained.

Next, an EFA was run on sub-model 3 consisting of all the concepts, including moderators, in the sub-model which examines the effect of politicization of gate reviews on demotivating gate experience. The specific concepts included in this sub-model were politicization of gate reviews, gate conditionality and demotivating gate experience. Since the item PolGateRev4 was dropped after the EFA for sub-model 2, this item was not included in the EFA for sub-model 3. Three factors with eigen-values above 1 emerged. The scree-plot also confirmed the existence of three factors. All items loaded on their respective concepts and cross-loadings were minimal (below 0.4) and thus all items were retained.

The last EFA was done on all the concepts, including moderators, in sub-model 4 which examines the effect of a demotivating gate experience on innovativeness of new products. This included the concepts demotivating gate experience, market turbulence, technological turbulence and innovativeness of new products. Four factors with eigenvalues above 1 emerged. The scree-plot also confirmed the existence of four factors. All items loaded on their respective concepts and cross-loadings were minimal (below 0.3). Since all factor loadings were above 0.5 (see Table 1), all the items were retained. 
6.2.2 Confirmatory Factor Analysis. Measures were further refined using confirmatory factor analysis (CFA) with EQS 6.1. The CFA model is considered a good fit if indices (such as CFI and NNFI) are above 0.90 , normed chi-square is below 5, and RMSEA is 0.10 or below (Hair et al. 2006). If the fit is not acceptable as per the above criteria, items need to be dropped until a good fit is obtained. To identify the items to be dropped to improve the fit, one needs to look for the items in the CFA output that have low factor loadings. In a model, items need to be dropped one by one until an acceptable fit is obtained.

As in the case of EFAs, four different CFAs were run, one for each sub-model in the framework, with all the concepts in the sub-model included ${ }^{3}$. Once again, the performance concept was excluded from this analysis because it is a formative measure (Diamantopoulos and Winklhofer 2001). Thus, CFA was not run on the fifth sub-model that captures the effect of innovativeness on performance. Each CFA was run as a congeneric measurement model consisting of a number of unidimensional concepts with each item/measure loading on only one concept and all concepts allowed to covary.

First, a four-factor confirmatory model consisting of all the concepts, including the moderators, used in sub-model 1 to examine the effect of a political organizational climate on power accumulation (i.e. political organizational climate, slack, centralization and power accumulation) was assessed. Since the item PowAccu2 was dropped after the EFA for sub-model 1 , this item was not included in the CFA for sub-model 1 . Several

\footnotetext{
${ }^{3}$ Considering the number of concepts and items for each concept in this dissertation, the sample size of 120 is not adequate for conducting a single CFA (Netemeyer, Bearden, and Sharma 2003).
} 
items such as slack3, centralization2, and centralization 5 were deleted to obtain a better

fit. The results (Table 2$)$ indicate good fit of the confirmatory measurement model $\left(\chi^{2}{ }_{183}\right.$ $=278.7, \mathrm{p}=.000$, normed $\left.\chi^{2}=1.52, \mathrm{CFI}=0.92, \mathrm{NNFI}=0.91, \mathrm{RMSEA}=0.07\right)$.

\section{Table 2}

\section{Sub-Model-Wise CFA Results}

Sub-Model 1: (Includes Political Organizational Climate, Slack, Centralization, \& Power Accumulation)

\begin{tabular}{|l|c|l|c|}
\hline Concept/Items & Item Loadings & Concept/Items & Item Loadings \\
\hline Pol.Org. Climate & .87 & Centralization & Cent 1 \\
\hline PolOrgCl1 & .87 & Cent 2 & .54 \\
PolOrgCl2 & .71 & Cent 3 & Deleted \\
PolOrgCl3 (R) & .83 & Cent 4 & .80 \\
PolOrgC14 & .62 & Cent 5 & .82 \\
PolOrgCl5 & .83 & Cent 6 & Deleted \\
PolOrgCl6 & .81 & Cent 7 & .77 \\
PolOrgCl7 & & Cent 8 & .69 \\
& .73 & Power Accumulation & .73 \\
\hline Slack & .72 & PowAccu1 (R) & .81 \\
Slack1 (R) & PowAccu3 (R) & .70 \\
Slack2 & .61 & PowAccu5 (R) & .67 \\
Slack3 & & PowAccu6 & .56 \\
Slack4 & PowAccu7 & .49 \\
\hline
\end{tabular}

Sub-Model 2: (Includes Power Accumulation, Strict Criteria, Objective Criteria, Entrepreneurial/Innovative Culture, \& Politicization of Gate Reviews)

\begin{tabular}{|l|l|l|l|}
\hline Power Accumulation & .87 & Entrepreneurial & \\
\hline PowAccu1 (R) & .69 & Innovative Culture & .51 \\
PowAccu3 (R) & .65 & InnovCul2 & .59 \\
PowAccu5 (R) & .50 & InnovCul3 & .96 \\
PowAccu6 & .46 & & \\
PowAccu7 & .86 & Politicization of Gate & \\
\hline Strict Criteria & .97 & Reviews & .62 \\
StrictCr1 & .77 & PolGateRev1 & .88 \\
StrictCr2 & & PolGateRev2 & .50 \\
StrictCr3 & .73 & & \\
\hline Objective Criteria & .68 & & \\
ObjCr1 (R) & .70 & & \\
ObjCr2 (R) & & & \\
ObjCr3 (R) & & \\
\hline
\end{tabular}




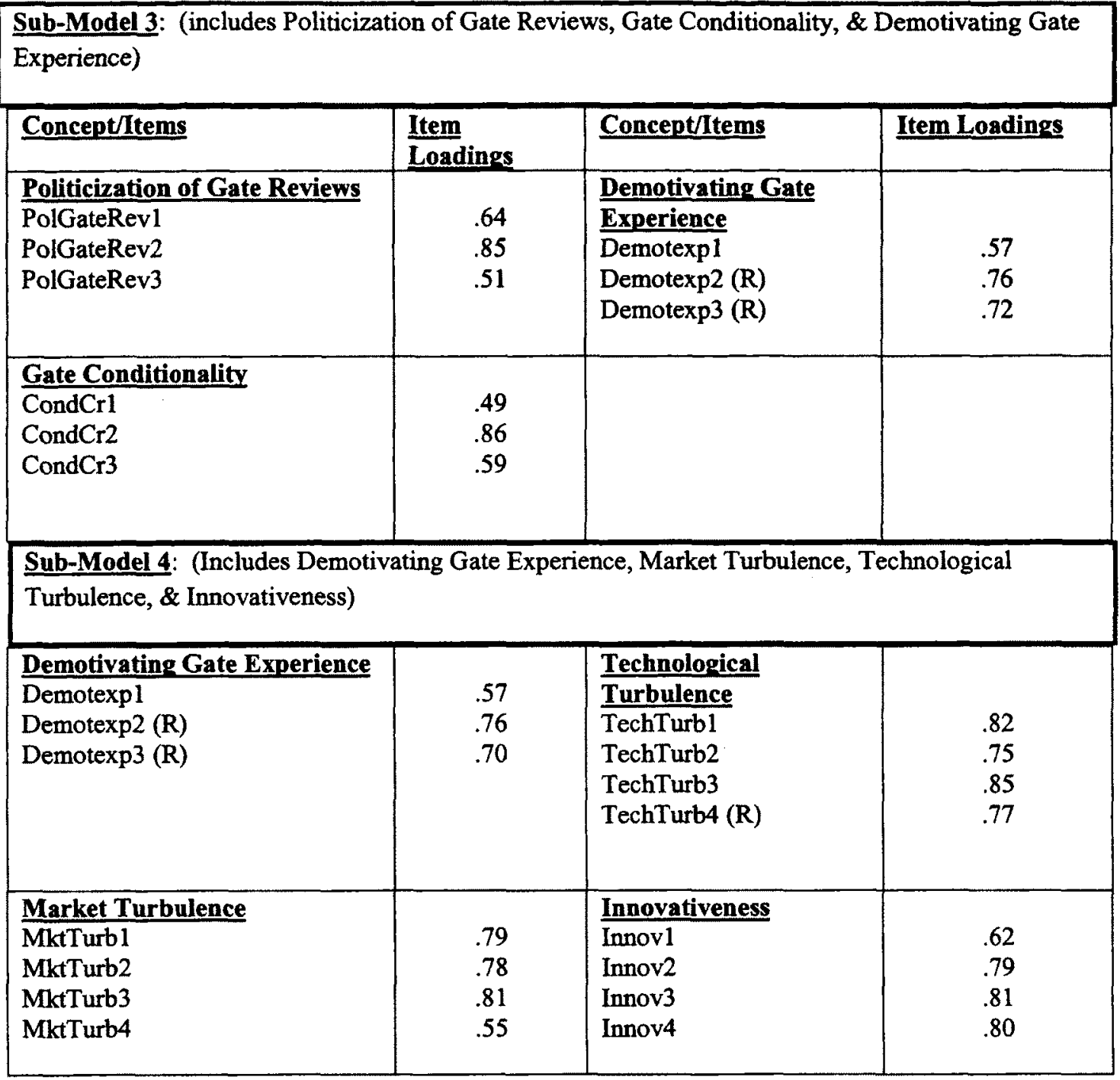

Then, confirmatory factor analysis was run on sub-model 2. This sub-model consisted of all the concepts, including the moderators, used to examine the effect of power accumulation on politicization of gate reviews (i.e. power accumulation, strict gate review criteria, objective criteria, entrepreneurial/innovative culture, and politicization of gate reviews). Since the item PolGateRev4 was dropped after the EFA for sub-model 2, this item was not included in the CFA for sub-model 2. The resulting confirmatory measurement model (See Table 2$)$ fitted well $\left(\chi^{2}{ }_{109}=161.3, p=.001\right.$, normed $\chi^{2}=1.48$, $\mathrm{CFI}=0.93, \mathrm{NNFI}=0.91, \mathrm{RMSEA}=0.06$ ) 
Then, a CFA was run on sub-model 3 consisting of all the concepts, including moderators, in the sub-model which examines the effect of politicization of the gate reviews on demotivating gate experience. The specific concepts included in this submodel were politicization of gate reviews, gate conditionality and demotivating gate experience. Results, shown in Table 2, indicate good fit of the confirmatory measurement model $\left(\chi^{2}{ }_{25}=45.67, p=.007\right.$, normed $\chi^{2}=1.83, \mathrm{CFI}=0.95, \mathrm{NNFI}=0.92$, RMSEA $=0.08$.

The last CFA was done on all the concepts, including moderators, in sub-model 4 which examines the effect of a demotivating gate experience on innovativeness of new products.

This included the concepts demotivating gate experience, market turbulence, technological turbulence and innovativeness of new products. The results, shown in Table 2, indicate good fit of the confirmatory measurement model with all the items being retained $\left(\chi^{2} 84=138.8, p=.000\right.$, normed $\chi^{2}=1.65, \mathrm{CFI}=0.93, \mathrm{NNFI}=0.91$, RMSEA $=0.07$ ).

Having conducted the factor analysis, the next step was to assess reliabilities and validities.

\subsection{Reliability and Validity}

6.3.1 Assessing Reliabilities. First, measure reliabilities (Cronbach's alpha) were computed. As the literature suggests, measures with alpha of 0.70 or above are usually 
considered reliable. However, coefficients as low as 0.50 may be accepted for a few concepts and for exploratory research (Nunnally 1978). All reliabilities were above 0.7 except for the measure of gate conditionality which has a reliability of 0.66 (Table 3 ). Thus, reliabilities are in the acceptable range.

\subsubsection{Convergent Validity. For convergent validity, all factor loadings should be} significant and this was the case with the loadings of the final measures. The loadings exceeded the minimum value of 0.5 suggested by Hair et al. (2005), except for power accumulation 7 and gate conditionalityl with loading estimates of 0.49 for each. These two items were retained in the model as they were close to the cut-off value and did not seem to harm the fit statistics for the CFA. In addition, reliabilities were high varying from .66 to .92 (Table 3). At the same time, the average variance extracted for all the measures was near or above 0.5 (see Table 5). Put together, the evidence supports the convergent validity of the measurement model.

To assess discriminant and nomological validity, correlations between summary measures of various concepts are needed. Thus, summary measures were created by averaging the item level scores for each measure and descriptive statistics were computed (see Table 3). Also, Pearson bi-variate correlations were computed among all the summary measures in this research (Table 4). The existence of correlations between the main effect variables (e.g., political organizational climate, power accumulation, politicization of the gate reviews, demotivating gate experience, innovativeness, and performance) in the expected direction provides initial support for the model. 
Table 3: Descriptive Statistics of Summary Measures

\begin{tabular}{|l|c|c|c|c|}
\hline Measure & \# of items & Mean & Std. Dev. & $\begin{array}{c}\text { Cronbach's } \\
\boldsymbol{a}\end{array}$ \\
\hline Political Organizational Climate & 7 & 4.40 & 1.31 & .92 \\
\hline Slack & 3 & 2.91 & 1.17 & .73 \\
\hline Centralization & 6 & 4.01 & 1.12 & .87 \\
\hline Power Accumulation & 5 & 4.29 & 1.00 & .77 \\
\hline Strict Review Criteria & 3 & 4.31 & 1.58 & .89 \\
\hline Objective Criteria & 3 & 3.53 & 1.26 & .74 \\
\hline Entrepreneurial/Innovative Culture & 3 & 3.70 & 1.28 & .74 \\
\hline Politicization of Gate Reviews & 3 & 3.88 & 1.27 & .71 \\
\hline Gate Conditionality & 3 & 4.74 & 1.07 & .66 \\
\hline Demotivating Gate Experience & 3 & 3.46 & 1.01 & .71 \\
\hline Market Turbulence & 4 & 4.20 & 1.16 & .82 \\
\hline Technological Turbulence & 4 & 4.64 & 1.36 & .87 \\
\hline Innovativeness & 4 & 3.83 & 1.26 & .84 \\
\hline Performance & 3 & 3.72 & 1.06 & $-\cdots$ \\
\hline
\end{tabular}

6.3.3 Discriminant Validity. To assess discriminant validity, the procedure suggested by Fornell and Larcker (1981) was used which is considered to be a strict test of discriminant validity (Grewal et al. 2004). Specifically, the average variance extracted for a measure needs to exceed the square of the correlation of the measure with other measures. All the measures passed this test, thereby establishing discriminant validity (Table 5 shows AVE and squared inter-measure correlations). 
Table 4: Pearson Bi-Variate Correlations

\begin{tabular}{|c|c|c|c|c|c|c|c|c|c|c|c|c|c|c|}
\hline Concept & 1 & 2 & 3 & 4 & 5 & 6 & 7 & 8 & 9 & 10 & 11 & 12 & 13 & 14 \\
\hline $\begin{array}{l}\text { 1. Political Organizational } \\
\text { Climate. }\end{array}$ & 1 & & & & & & & & & & & & & \\
\hline 2. Slack & $-.22^{*}$ & 1 & & & & & & & & & & & & \\
\hline 3. Centralization & $.42^{* *}$ & $-.25^{* *}$ & 1 & & & & & & & & & & & \\
\hline 4. Power Accumulation & $.48^{* *}$ & $-.31^{* *}$ & $.46^{* *}$ & 1 & & & & & & & & & & \\
\hline 5. Strict Criteria & -.01 & .07 & .04 & -.12 & 1 & & & & & & & & & \\
\hline 6. Objective Criteria & -.11 & $.20^{*}$ & -.00 & $-.21^{*}$ & $.53^{* *}$ & 1 & & & & & & & & \\
\hline $\begin{array}{l}\text { 7. Entrepreneurial/ } \\
\text { Innovative Culture }\end{array}$ & $-.46^{* \prime}$ & $.37^{* *}$ & $-.47^{* *}$ & $-.41^{* *}$ & -.06 & .03 & 1 & & & & & & & \\
\hline $\begin{array}{l}\text { 8. Politicization of } \\
\text { Gate Reviews }\end{array}$ & $.48^{* *}$ & -.11 & $.23^{* *}$ & $.36^{* *}$ & -.17 & $-.24^{* *}$ & $-.20^{*}$ & 1 & & & & & & \\
\hline 9. Gate Conditionality & -.05 & -.12 & .10 & -.06 & $-.20^{*}$ & $-.40^{* *}$ & .08 & -.05 & 1 & & & & & \\
\hline $\begin{array}{l}\text { 10. Demotivating Gate } \\
\text { Experience }\end{array}$ & $.32^{* *}$ & $-.27^{* *}$ & $.29^{* *}$ & $.31^{* *}$ & -.11 & -.01 & $-.42^{* *}$ & $.38^{* *}$ & -.05 & 1 & & & & \\
\hline 11. Market Turbulence & .13 & -.10 & .17 & -.03 & .16 & .06 & -.15 & .06 & -.08 & .15 & 1 & & & \\
\hline $\begin{array}{l}\text { 12. Technological. } \\
\text { Turbulence }\end{array}$ & -.06 & .04 & .09 & -.07 & $.18^{*}$ & .04 & .14 & .05 & -.12 & .10 & $.27^{* *}$ & 1 & & \\
\hline 13. Innovativeness & $-.22^{*}$ & $.27^{* *}$ & -.15 & $-.26^{* *}$ & $.23^{\circ}$ & $.22^{*}$ & $.45^{* *}$ & -.11 & -.06 & $-.24^{* *}$ & .10 & $.34^{* *}$ & 1 & \\
\hline 14. Performance & $-.23^{*}$ & $.36^{* *}$ & $-.22^{*}$ & $-.29^{* *}$ & .11 & $.26^{* *}$ & $.43^{* *}$ & -.13 & .07 & $-.20^{*}$ & $-.19^{*}$ & -.03 & $.43^{* *}$ & 1 \\
\hline
\end{tabular}

Significant at $p<0.05, \quad$ Significant at $p<0.01 \quad$ (two tailed) 
Table 5: Test of Discriminant Validity

\begin{tabular}{|c|c|c|c|c|c|c|c|c|c|c|c|c|c|c|}
\hline Concept & 1 & 2 & 3 & 4 & 5 & 6 & 7 & 8 & 9 & 10 & 11 & 12 & 13 & 14 \\
\hline 1. Political Organizational Climate & .63 & & & & & & & & & & & & & \\
\hline 2. Slack & .05 & .48 & & & & & & & & & & & & \\
\hline 3. Centralization & .18 & .06 & .53 & & & & & & & & & & & \\
\hline 4. Power Accumulation & .23 & .10 & .21 & .43 & & & & & & & & & & \\
\hline 5. Strict Criteria & .00 & .01 & .00 & .01 & .76 & & & & & & & & & \\
\hline 6. Objective Criteria & .01 & .04 & .00 & .04 & .28 & .49 & & & & & & & & \\
\hline \begin{tabular}{|l|} 
7. Entrepreneurial/Innovative \\
Culture \\
\end{tabular} & .21 & .14 & .22 & .17 & .00 & .00 & .50 & & & & & & & \\
\hline 8. Politicization of Gate Reviews & .23 & .01 & .05 & .13 & .03 & .06 & .04 & .47 & & & & & & \\
\hline 9. Gate Conditionality & .00 & .01 & .01 & .00 & .04 & .16 & .06 & .03 & .44 & & & & & \\
\hline 10. Demotivating Gate Experience & .10 & .07 & .08 & .10 & .01 & .00 & .18 & .14 & .03 & .47 & & & & \\
\hline 11. Market Turbulence & .02 & .01 & .03 & .00 & .03 & .00 & .02 & .00 & .01 & .02 & .54 & & & \\
\hline 12. Technological Turbulence & .00 & .00 & .01 & .01 & .03 & .00 & .02 & .00 & .01 & .01 & .07 & .65 & & \\
\hline 13. Innovativeness & .05 & .07 & .02 & .07 & .05 & .05 & .20 & .01 & .00 & .06 & .01 & .12 & .58 & \\
\hline 14. Performance & .05 & .13 & .05 & .08 & .01 & .07 & .19 & .02 & .00 & .04 & .04 & .00 & .19 & - \\
\hline
\end{tabular}

Numbers in off-diagonal cells are squared correlations and diagonal numbers are average variance extracted, except for performance, which is a formative measure. 
6.3. 4 Nomological Validity. A concept has nomological validity if it relates to other concepts in a way that is consistent with a priori expectations (Peter 1981). The correlations among concepts suggest that the concepts in the model are related to one another in the manner predicted by theory (Table 4). For example, as expected there is a positive correlation between political organizational climate and power accumulation, power accumulation and the politicization of gate reviews, the politicization of gate reviews and demotivating gate experience, and innovativeness and performance. Similarly, as predicted, there is a negative correlation between demotivating gate experience and innovativeness. Finally, the concepts that were not expected to be correlated have zero correlation between them. For example, as expected, political organizational climate is not correlated with strict and objective criteria. Thus, the evidence supports nomological validity of various concepts.

Having assessed reliability and validity, checks were conducted for biases due to nonresponse, different modes of responding, and different backgrounds of the respondents.

\subsection{Check for Biases}

6.4.1 Non-Response Bias. To assess the degree of non-response bias, responses were divided into two groups - responses received before reminders and those received after reminders. Late responders are often believed to be more similar to non-responders than are early responders. Therefore, these calculations were made under the assumption that those who responded late were similar to non-respondents (Armstrong and Overton 
1977). T-tests were conducted to examine the possible difference between groups using mean responses to each of the concepts included in the research. There were no significant differences between groups (Appendix 3 at the end of the document), suggesting, by extrapolation that no or few significant differences would exist between non-responders and responders.

\subsubsection{Differences between Different Modes of Responding. Responses were received} either through mail or fax. To test for possible differences on the means of the concept scores between those who responded via mail and those who faxed their responses, t-tests were conducted. No differences were found between the groups (Appendix 3).

\subsubsection{Differences between Respondents from Different Backgrounds. The project} managers who completed the survey came from four different backgrounds: marketing, product development and R\&D, general management, and manufacturing. To test for differences in mean responses on the concepts in this study between managers from different backgrounds, one-way ANOVA was done. None of the results were significant. Thus it can be concluded that there were no differences in responses between managers from different backgrounds and hence the responses could be pooled for analysis purposes (Appendix 3).

With these checks for biases, all the work on measure development was complete and all the requirements (e.g., concept unidimensionality through EFA and CFA, reliability checks, validity checks, and response bias checks) had been met. Thus, the measures 
were ready for hypotheses testing. Thereafter, hypotheses were tested using moderated regression analysis. Also, tests of mediation were done.

\subsection{Hypotheses Testing}

Hypotheses 1 to 12 were tested using moderated regression analysis. Each of the submodels of the conceptual framework was tested separately. Hypothesis 13 was tested using simple regression. A serial approach to testing for moderation was adopted in the case of each sub-model, as suggested by Sharma, Durand, and Gur-Arie (1981). This approach involves comparing three regression equations, one with only the main effect of the predictor $\mathrm{X}$ (equation 1 below), another with the main effects of the predictor $\mathrm{X}$ and the moderator $\mathrm{Z}$ (equation 2), and the third with the main effects of the predictor $\mathrm{X}$, moderator $\mathrm{Z}$, and the interaction $\mathrm{XZ}$ (equation 3). In these equations, $\mathrm{Y}$ is the dependent variable.

$\mathrm{Y}=\mathrm{a}+\mathrm{b}_{1} \mathrm{X}$

$\mathrm{Y}=\mathrm{a}+\mathrm{b}_{1} \mathrm{X}+\mathrm{b}_{2} \mathrm{Z}$

$\mathrm{Y}=\mathrm{a}+\mathrm{b}_{1} \mathrm{X}+\mathrm{b}_{2} \mathrm{Z}+\mathrm{b}_{3} \mathrm{XZ}$

If equations 2 and 3 are not significantly different (i.e. $b_{3}=0 ; b_{2} \neq 0$ ), $Z$ is not a moderator variable, but only an independent predictor variable. If equations 1 and 2 are not different but are different from equation 3 , then $\mathrm{Z}$ is a pure moderator (i.e. $b_{2}=0 ; b_{3} \neq$ 0 ). For $\mathrm{Z}$ to be called a quasi-moderator, equations 1, 2, and 3 should be different from 
each other (i.e. $b_{2} \neq b_{3} \neq 0$ ). The significance test for the difference between two models is based on the "extra sums of squares" principle or an F-test (Sharma et al. 1981). The F statistic is computed using the formula (Jaccard, Turrisi, and Wan 1990):

$\mathrm{F}_{(\mathrm{K} 2-\mathrm{K} 1 ; \mathrm{N}-\mathrm{K} 2-1)}=\left(\left(\mathrm{R}_{22}-\mathrm{R}_{12}\right) /\left(\mathrm{K}_{2}-\mathrm{K}_{1}\right)\right) /\left(\left(1-\mathrm{R}_{22}\right) /\left(\mathrm{N}-\mathrm{K}_{2}-1\right)\right)$

$R_{12}$ and $R_{22}$ are the $R$-squares for the first and second models. $K_{1}$ and $K_{2}$ are the number of predictors in the first and the second model, and $\mathrm{N}$ is the number of observations.

The independent and moderator variables were mean-centered prior to the formation of interaction terms to minimize multicollinearity among interaction terms and their constituent terms in the regression model (Aiken and West 1991; Jaccard, Turrisi, and Wan 1990).

\subsubsection{Test of Sub-Model 1}

Sub-model 1 examines the effect of a political organizational climate (PolOrgCl) on power accumulation (Power Accum), and the moderating effect of slack and centralization. The three regression equations being compared are:

Power Accum $=a+b_{1}$ PolOrgCl

Power Accum $=a+b_{1}$ PolOrgCl $+b_{2}$ Slack $+b_{3}$ Centralization

Power Accum $=a+b_{1}$ PolOrgCl $+b_{2}$ Slack $+b_{3}$ Centralization $+b_{4}$ PolOrgCl X Slack + $\mathrm{b}_{5}$ PolOrgCl X Centralization 
First power accumulation was regressed on political organizational climate (equation 4). This explained $22 \%$ of the variance in power accumulation $(\mathrm{F}=34.9, \mathrm{p}=.00$, Adjusted $\mathrm{R}^{2}=.22$ ). As the regression results indicate (Table 6, column 1), political organizational climate is a significant predictor of power accumulation $\left(b=.31, \beta_{1}=.48, p=.00\right)$.

Thus, the more political the organizational climate, the more power accumulation occurs.

Table 6

Regression Results (Sub-Model 1)

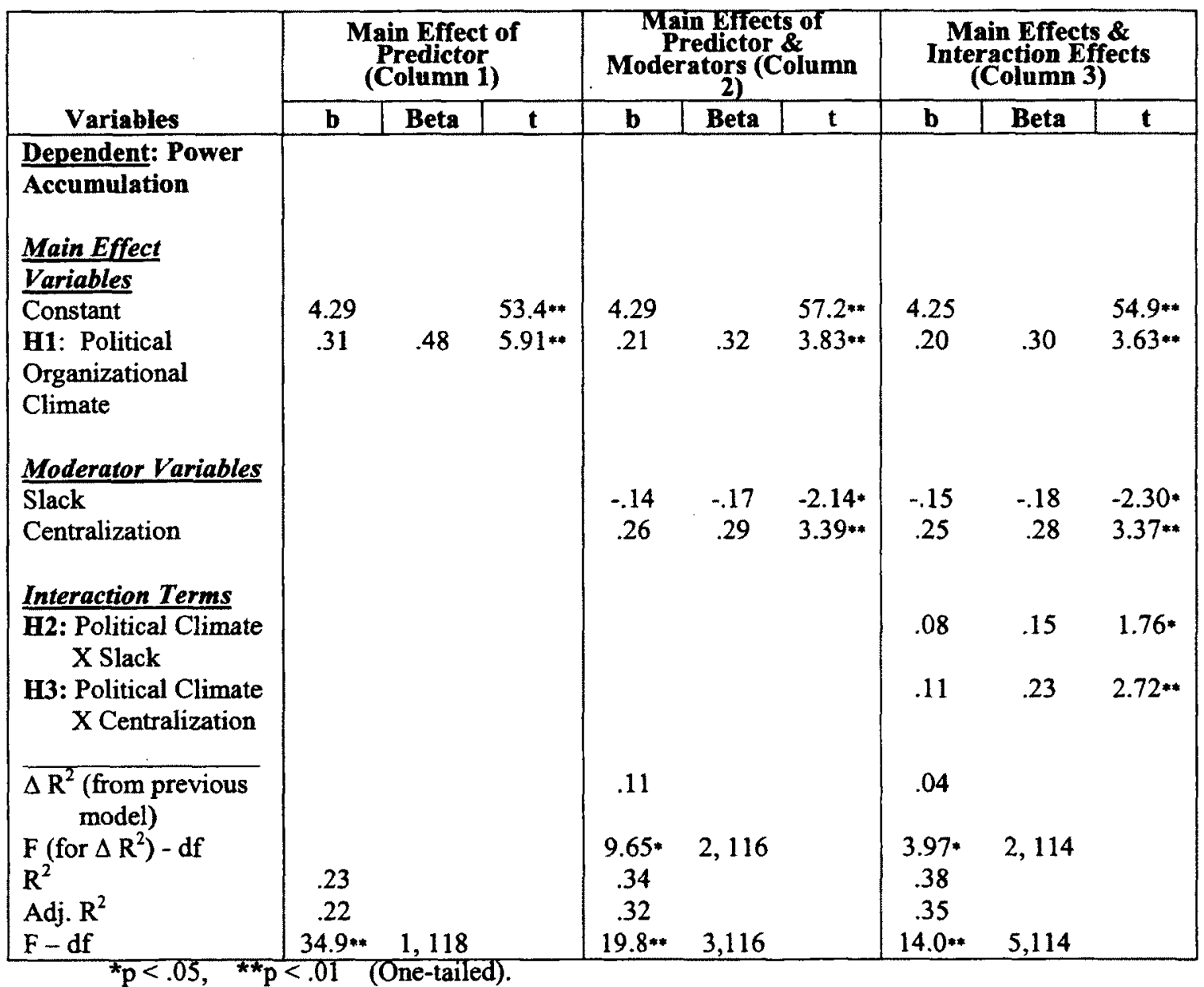

In the next regression, power accumulation was regressed on political organizational climate, slack and centralization (equation 5). This regression was significant and 
explained $32 \%$ of the variance in power accumulation $\left(F=19.8, p=.00\right.$, Adjusted $R^{2}=$ .32). The change in $R^{2}$ between equations 4 and 5 is .11 and the F-test for the difference between the two models is significant $(\mathrm{F}=9.65, \mathrm{df}=2,116 ; \mathrm{p}<.05)$. As the regression results indicate, slack $\left(b_{2}=-.14, \beta_{2}=-.17, p=.02\right)$ and centralization $\left(b_{3}=.26, \beta_{3}=.29\right.$, $\mathrm{p}=.00$ ) are making a contribution to the model over and above the contribution made by political organizational climate in predicting power accumulation (Table 6, column 2). Thus, slack and centralization are positively associated with power accumulation.

In the third regression, power accumulation was regressed on political organizational climate, slack, centralization and the interaction between political organizational climate and slack, as well as the interaction of political organizational climate with centralization (equation 6). This regression was significant and explained $35 \%$ of the variance in power accumulation $\left(F=14.0, p=.00\right.$, Adjusted $\left.R^{2}=.35\right)$. Variance inflation factors for independent variables in the model were also obtained and all were well below 10 , the value above which there should be concern for multicollinearity (Myers 1986). The difference in $R^{2}$ between equations 5 and 6 is .04, and the F-test for the difference between the two is significant $(\mathrm{F}=3.97, \mathrm{df}=2,114 ; \mathrm{p}<.05)$. As the regression results indicate, the two interaction effects between political organizational climate and the moderators slack $\left(b_{4}=.08, \beta_{4}=.15, p=.04\right)$ and centralization $\left(b_{5}=.11, \beta_{5}=.23, p=\right.$ .00 ) are making a significant contribution to the model beyond the contribution made by political organizational climate, slack, and centralization in predicting power accumulation (Table 6, column 3). The main effects of political organizational climate, slack, and centralization are still significant in the model. 
In other words, a political organizational climate is associated with managers' accumulation of more power $\left(b_{1}=.20, \beta_{1}=.30, p=.00\right)$. Thus Hypothesis 1 is supported. Also, the interaction effects between political organizational climate and slack $\left(b_{4}=.08\right.$, $\left.\beta_{4}=.15, p=.04\right)$ and between political organizational climate and centralization $\left(b_{5}=\right.$ $.11, \beta_{5}=.23, p=.00$ ) are significant and positive, providing support for Hypothesis 2 and 3 respectively (Table 6, column 3). This indicates that in the presence of both slack and centralization, there is increased association of a political organizational climate and power accumulation.

While the interaction between slack and a political organizational climate is positively associated with power accumulation, the direct association of slack with power accumulation is negative. This suggests that slack by itself discourages senior managers from acquiring power, but when the climate is political, senior managers use available slack resources to acquire power.

The effect size for the regression (equation 6) was 0.61 which is considered large. This indicates that a strong relationship exists between these predictors as modeled and power accumulation, the outcome variable (Cohen 1988).

Further, since the two interaction terms are significant and both the moderators slack and centralization have a significant main effect on the dependent variable power accumulation, it can be concluded that slack and centralization are quasi-moderators (Sharma, Durand and Gur-Arie 1981). 


\subsubsection{Inflection Point for the Relationship between Power Accumulation and Political}

Organizational Climate. To understand any changes in the nature of the association between political organizational climate (independent variable) and power accumulation (dependent variable) over the range of the moderator variables (slack or centralization), the method suggested by Schoonhoven (1981) and used by Harris (2001) is employed. As per this method, the aim is to obtain the inflection point at which the relationship between political organizational climate and power accumulation changes with change in the moderator variable. For this purpose, a partial derivative of equation 6 above is obtained with respect to political organizational climate. This yields:

$\mathrm{d}$ Power Accumulation $/ \mathrm{d}$ Pol. Org. Climate $=\mathrm{b}_{1}+\mathrm{b}_{4}$ Slack $+\mathrm{b}_{5}$ Centralization ..... (7)

To obtain the inflection point, this equation is set equal to zero. Thus,

$d$ Power Accumulation $/ d$ Pol. Org. Climate $=b_{1}+b_{4}$ Slack $+b_{5}$ Centralization $=0 \ldots$

Inflection Point for the Moderator Centralization: Equation 8 is first interpreted for the moderator centralization. To do so, the other moderator, slack, is held at its mid-point because the variables in the regression have been mean centered. When variables are mean- centered, the interpretation of coefficients changes. In a non-mean-centered model, the effects are interpreted as constant effects. For example, in a model $y=a_{0}+a_{1}$ $X_{1}+a_{2} X_{2}$, the coefficient of $X_{1}$ represents the effect of the variable $X_{1}$ on $Y$, holding all other variables in the model constant. Instead, the coefficient of $X_{1}$ in a mean-centered 
model is interpreted as the regression of $\mathrm{Y}$ on $\mathrm{X}_{1}$ when the variable $\mathrm{X}_{2}$ is at its mean value (Aiken and West 1991; Jaccard, Turrisi, and Wan 1990).

Since the data is mean-centered, the mean of slack is zero. Equation 8 becomes:

$\mathrm{d}$ power accumulation $/ \mathrm{d}$ Pol. Org. Climate $=\mathrm{b}_{1}+\mathrm{b}_{5}$ Centralization $=0$

Or, Centralization $=-b_{1} / b_{5}$

As suggested by Schoonhoven (1981) and Harris (2001), for obtaining the inflection point unstandardized regression coefficients are used. Substituting values of $b_{1}$ (the coefficient of political organizational climate) and $b_{5}$ (coefficient of the interaction term between political organizational climate and centralization) in equation 10 , from the regression output (Table 6, column 3),

Centralization $=-0.20 / 0.11=-1.82$

This value of centralization represents the inflection point for the relationship between political organizational climate and power accumulation (Harris 2001; Schoonhoven 1981). In other words, when the value of mean-centered centralization is above -1.82 or non-mean-centered average value is above 2.19 (obtained by adding the mean of centralization 4.01 to the mean-centered value -1.82 ), the relationship between political organizational climate and power accumulation is positive and below this value the relationship is negative (when slack is at its mid-point). 
The above conclusion is based on equation 11 below, which in turn is based on equation 9 without equating it to zero. When the values of the coefficients are included:

$\mathrm{d}$ power accumulation $/ \mathrm{d}$ Pol. Org. Climate $=.20+.11$ Centralization

Two values of centralization were inserted in equation 11, one at a time: +2.24 (which is +2 standard deviations from the mean) and -2.24 (which is -2 standard deviations).

Centralization values of $+/-2$ standard deviations were chosen to generate two solutions to equation 11, one that is above the inflection point and the other below it. With centralization equal to +2.24 (i.e. above the inflection point of -1.82 ) the value of equation 11 is +.45 and with centralization equal to -2.24 (i.e. below the inflection point of -1.82 ) the value of the equation is -.05 . Thus, the relationship between political organizational climate and power accumulation is indeed positive above the inflection point and negative below it.

As centralization increases, the positive association between political organizational climate and power accumulation becomes more positive. Before the inflection point, the relationship between political organizational climate and power accumulation is negative, but that negative effect decreases with increase in centralization. Beyond the inflection point, the relationship turns positive. About $95 \%$ organizations lie to the right of the inflection point, i.e. the range in which dPower Accum/dPol Org Climate is positive ${ }^{4}$.

\footnotetext{
${ }^{4}$ The inflection point of -1.82 is $-1.63 \mathrm{Std}$. Dev. from the mid-point of Centralization (where Std. Dev is 1.12). Given that centralization data is near normal (as shown by the test of normality conducted earlier), from the normal table, about $95 \%$ of the responding organizations lie to the right of the inflection point.
} 
Inflection Point for the Moderator Slack. Next, equation 8 is interpreted for the moderator slack. To do so the other moderator centralization is held at its mid point. Again, since the data is mean centered, the mean of centralization is zero. Therefore, equation 8 becomes:

d power accumulation $/ d$ Pol. Org. Climate $=b_{1}+b_{4}$ Slack $=0 \ldots \ldots$ (12)

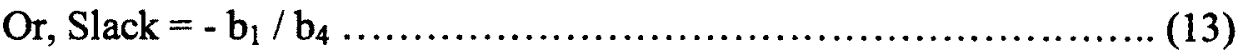

Substituting values of $b_{1}$ (coefficient of political organizational climate) and $b_{4}$ (coefficient of the interaction term between political organizational climate and slack) in equation 13, from the regression output (Table 6, column 3),

Slack $=-0.20 / 0.08=-2.5$

Again, this value of slack represents the inflection point for the relationship between political organizational climate and power accumulation. When the value of meancentered slack is above -2.5 or non-mean-centered average value is above 0.41 (obtained by adding the mean of slack 2.91 to the mean-centered value -2.5 ), the relationship between political organizational climate and power accumulation is positive and below this value the relationship is negative (when centralization is at its mid-point).

This conclusion is based on equation 14 below which in turn is based on equation 12 without equating it to zero. When the values of the coefficients are included: 
Two values of slack were inserted in equation 14 , one at a time: +3.51 (which is +3 standard deviations from the mean) and -3.51 (which is -3 standard deviations). Slack values of $+/-3$ standard deviations were chosen to generate two solutions to equation 14 , one that is above the inflection point and the other below it. With slack equal to +3.51 (i.e. above the inflection point of -2.5 ) the value of equation 14 is +.48 and with slack equal to -3.51 (i.e. below the inflection point of -2.5) the value of the equation is .08. Thus the relationship between political organizational climate and power accumulation is indeed positive above the inflection point and negative below it.

As slack goes up, the positive association between political organizational climate and power accumulation increases. Before the inflection point, the relationship between political organizational climate and power accumulation is negative, but that negative effect decreases with an increase in slack. Beyond the inflection point, this relationship turns positive. Among the responding organizations, about $98 \%$ lie to the right of the inflection point, i.e. the range in which dPowerAccum/dPol Org Climate is positive ${ }^{5}$.

Overall, the analysis for Sub-model 1 shows that the more political the organizational climate, the more power accumulation occurs. As centralization and slack go up, the association between political organizational climate and power accumulation increases.

\footnotetext{
${ }^{5}$ The inflection point of -2.5 is -2.14 Std. Dev. from the mid-point of Slack (where Std. Dev is 1.17). Given that slack data is near normal (as shown by the test of normality conducted earlier), from the normal table, $98.4 \%$ of the responding organizations lie to the right of the inflection point.
} 
The positive moderating effects of centralization and slack are confirmed by the inflection point analyses. The inflection point analysis also suggests that a majority of responding organizations (95\%) lie in the range where an increase in centralization is associated with a positive relationship between a political organizational climate and power accumulation. Similarly, $98 \%$ of responding organizations lie in the range where an increase in slack is associated with a positive relationship between a political organizational climate and power accumulation.

\subsubsection{Test of Sub-Model 2}

Sub-model 2 examines the effect of power accumulation (Power Accum) on the politicization of the gate reviews (PolGateRev), and the moderating effects of strict review criteria (Strict $\mathrm{Cr}$ ), objective review criteria (Objective $\mathrm{Cr}$ ), and an entrepreneurial/innovative culture ( $\mathrm{Inn} \mathrm{Cul})$. The regression equations are:

PolGate Rev $=a+b_{1}$ Power Accum

PolGate Rev $=a+b_{1}$ Power Accum $+b_{2}$ Strict $C r+b_{3}$ Objective $\mathrm{Cr}+b_{4} \operatorname{Inn} \mathrm{Cul}$

Pol Gate Rev $=a+b_{1}$ Power Accum $+b_{2}$ Strict $C r+b_{3}$ Objective $C r+b_{4}$ Inn Cul + $b_{5}$ PowerAccum X StrictCr $+b_{6}$ Power Accum X Objective Cr. + $b_{7}$ PowerAccum X Inn Cul

To test this model, first politicization of reviews was regressed on power accumulation (Equation 15). This explained $12 \%$ of the variance in politicization of gate reviews $(\mathrm{F}=$ 
17.2, $\mathrm{p}=.00$, Adjusted $\mathrm{R}^{2}=.12$ ). As shown in Table 7 column 1 , power accumulation is significantly related with the politicization of gate reviews $\left(b_{1}=.45, \beta_{1}=.36, p=.00\right)$.

Thus, the more the power accumulation, the more is the politicization of gate reviews.

Table 7

Regression Results (Sub-Model 2)

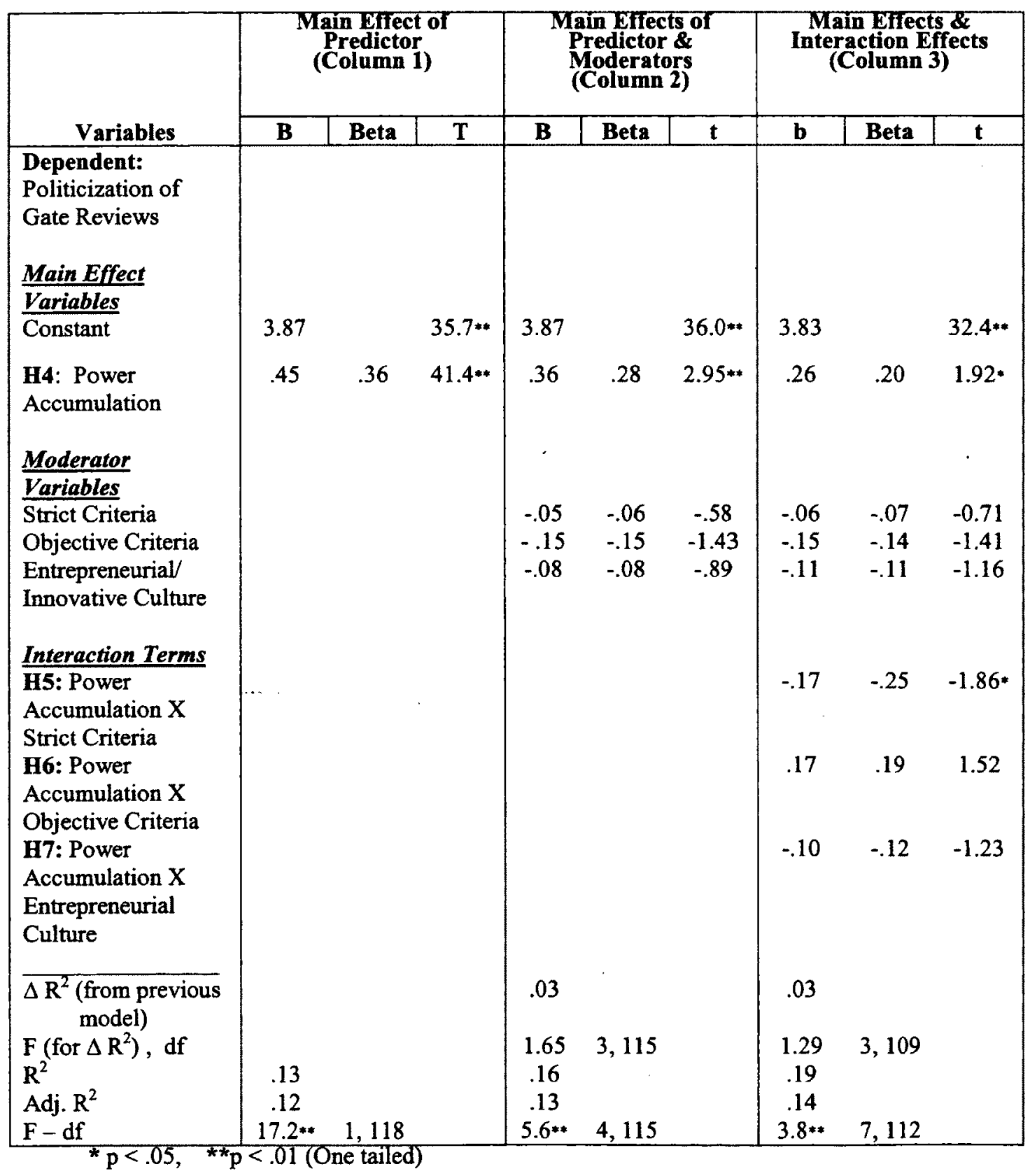


Next, politicization of gate reviews was regressed on power accumulation, strict gate review criteria, objective gate review criteria, and an entrepreneurial/innovative culture (equation 16). This regression was also significant and explained $13.4 \%$ of the variance in politicization of gate reviews $\left(F=5.6, p=.00\right.$, Adjusted $\left.R^{2}=13.4\right)$. The change in $R^{2}$ between the first and the second equations is .03 . The F-test for the difference between the two models (equations 15 and 16 ) is not significant $(F=1.65, \mathrm{df}=3,115 ; \mathrm{p}>.05$ ). Thus, strict review criteria, objective review criteria and an entrepreneurial/innovative culture are not making a contribution to the model over and above the contribution made by power accumulation in predicting politicization of gate reviews (Table 7 , column 2 ).

In the third regression, politicization of gate reviews was regressed on power accumulation, strict review criteria, objective review criteria, entrepreneurial/innovative culture, the interaction of power accumulation and strict review criteria, interaction of power accumulation with objective review criteria, and the interaction of power accumulation with entrepreneurial/innovative culture (equation 17). This regression was significant (Table 7, column 3) and explained $14 \%$ of the variance in politicization of gate reviews $\left(F=3.8, p=.00\right.$, Adjusted $\left.R^{2}=14.1\right)$. Variance inflation factors for independent variables in the model were also obtained and all were well below 10 , the value above which there should be concern for multi-collinearity (Myers 1986). However, the F-test for the difference between the two regressions (equations 16 and 17) is not significant $(F=1.29, \mathrm{df}=3,109 ; \mathrm{p}>.05)$. Thus the interaction terms are not making a significant contribution to the model. 
Since the F-tests for the difference between regression equations 15 and 16 and between 16 and 17 were both not significant, it can be concluded that the moderators (strict review criteria, objective review criteria, and entrepreneurial/innovative culture) neither lead to politicization of gate reviews nor their interaction with power accumulation has any effect on politicization of gate reviews. Only power accumulation leads to an increase in politicization of gate reviews $\left(b_{1}=.26, \beta_{1}=.20, p=.03\right)$. Thus, the association between power accumulation and politicization of gate reviews is not affected by the strictness of review criteria, the objectivity of the review criteria, or the entrepreneurial/innovative culture of the organization. Hence, there is support for hypothesis 4, but hypotheses 5,6 , and 7 are not supported.

Yet, the effect size for the regression (equation 15) was 0.15 which is considered medium suggesting that a moderate relationship exists between the predictor and politicization of gate reviews - the outcome variable (Cohen 1988).

Further, since the interaction terms are not significant and the moderators do not have a significant main effect on the dependent variable, it can be concluded that strict criteria, objective criteria, and entrepreneurial/innovative culture are neither moderators nor predictors (Sharma, Durand, and Gur-Arie 1981).

Overall, the more the power accumulation, the greater is the politicization of gate reviews. However, the moderators strict criteria, objective criteria, and an 
entrepreneurial/innovative culture have neither an association with politicization nor any bearing on the association between power accumulation and politicization.

\subsubsection{Test of Sub-Model 3}

Sub-model 3 examines the effect of politicization of gate reviews (Pol Gate Rev) on demotivating gate experience (Demot Gate Exp) for managers taking their new products through the gate review process and the moderating effect of gate conditionality (Gate Cond). The three regression equations being compared are:

DemotGateExp $=a+b_{1}$ Pol Gate Rev

DemotGateExp $=a+b_{1}$ Pol Gate Rev $+b_{2}$ Gate Cond

DemotGateExp $=a+b_{1}$ Pol Gate Rev $+b_{2}$ Gate Cond $+b_{3}$ Pol Gate RevX Gate Cond..(20)

First, demotivating gate experience was regressed on politicization of gate reviews (equation 18). This regression was significant and explained $13 \%$ of the variance in demotivating gate experience $\left(F=19.3, p=.00\right.$, Adjusted $\mathrm{R}^{2}=.13$ ). As Table 8 (column 1) shows, politicization of gate reviews had a significant relationship with demotivating gate experience $\left(b_{1}=.30, \beta_{1}=.38, p=.00\right)$.

In the next regression, demotivating gate experience was regressed on politicization of gate reviews and gate conditionality (equation 19). This regression was significant and explained $13 \%$ of the variance in demotivating gate experience $(F=9.6, p=.00$, Adjusted 
$\mathrm{R}^{2}=.13$ - Table 8 , column 2). The change in $\mathrm{R}^{2}$ between equations 18 and 19 is .001 .

The F-test for the difference between the two models is not significant $(F=0.14, \mathrm{df}=1$, $117 ; \mathrm{p}>.05)$. Thus, gate conditionality does not make a contribution to the model over and above the contribution made by politicization of gate reviews in predicting demotivating gate experience.

Table 8

Regression Results (Sub-Model 3)

\begin{tabular}{|c|c|c|c|c|c|c|c|c|c|}
\hline \multirow[b]{2}{*}{ Variables } & \multicolumn{3}{|c|}{$\begin{array}{l}\text { Main Effect of } \\
\text { Predictor (Column 1) }\end{array}$} & \multicolumn{3}{|c|}{$\begin{array}{l}\text { Main Effects of } \\
\text { Predictor \& } \\
\text { Moderators } \\
\text { (Column 2) }\end{array}$} & \multicolumn{3}{|c|}{$\begin{array}{c}\text { Main Effects \& } \\
\text { interaction Effects } \\
\text { (Column 3) }\end{array}$} \\
\hline & b & Beta & $\mathbf{T}$ & B & Beta & $t$ & b & Beta & $t$ \\
\hline $\begin{array}{l}\text { Dependent: } \\
\text { Demotivating } \\
\text { Gate Experience } \\
\text { Main Effect } \\
\text { Variables }\end{array}$ & & & & & & & & & \\
\hline$\overline{\text { Constant }}$ & 3.46 & & $40.2 * *$ & 3.46 & & $40.1 * *$ & 3.46 & & $39.9 * *$ \\
\hline $\begin{array}{l}\text { H8: Politicization } \\
\text { of Gate Reviews } \\
\frac{\text { Moderator }}{\text { Variables }}\end{array}$ & .30 & .38 & $4.39 * *$ & .30 & .37 & $4.36 * *$ & .30 & .38 & $4.37 * *$ \\
\hline $\begin{array}{l}\text { Gate } \\
\text { Conditionality }\end{array}$ & & & & -.03 & -.03 & -.32 & -.03 & -.03 & -.39 \\
\hline $\begin{array}{l}\text { Interaction Terms } \\
\text { H9: Politicized } \\
\text { Gate Reviews X } \\
\text { Conditionality }\end{array}$ & & & & & & & -.03 & -.04 & -.43 \\
\hline $\begin{array}{l}\overline{\Delta R^{2} \text { (from }} \\
\text { previous model) }\end{array}$ & & & & .001 & & & .00 & & \\
\hline $\begin{array}{l}F\left(\text { for } \Delta R^{2}\right)-d f \\
R^{2}\end{array}$ & .14 & & & $\begin{array}{l}.14 \\
.14\end{array}$ & 1,117 & & $\begin{array}{l}.14 \\
.14\end{array}$ & 1,116 & \\
\hline Adj. $R^{2}$ & .13 & & & .13 & & & .12 & & \\
\hline$F-d f$ & $19.3 * *$ & 1,118 & & $9.6 * *$ & 2,117 & & $6.5 * *$ & 3,116 & \\
\hline
\end{tabular}


In the third regression, demotivating gate experience was regressed on politicization of gate reviews, gate conditionality, and the interaction between politicization of gate reviews and gate conditionality (equation 20). This regression was significant and explained $12 \%$ of the variance in demotivating gate experience $(F=6.5, p=.00$, Adjusted $\mathrm{R}^{2}=.12$ - Table 8, column 3). Variance inflation factors for independent variables in the model were also obtained and all were well below 10 , the value above which there should be concern for multicollinearity (Myers 1986). The F-test for the difference between the two models (equations 19 and 20 ) is not significant $(F=.14, \mathrm{df}=1,116 ; \mathrm{p}>.05$ ). Thus, gate conditionality and its interaction with politicization of gate reviews are not making a significant contribution to the model over and above the contribution made by politicization of gate reviews in predicting demotivating gate experience.

As the above regression suggests, politicization of gate reviews leads to a demotivating gate experience for product development managers taking their products through gate reviews $\left(b_{1}=.30, \beta_{1}=.38, p=.00\right)$. Thus hypothesis 8 is supported. However, hypothesis 9 which predicted that gate conditionality would reduce the adverse effect of politicization of gate reviews on the demotivating experience of product development managers is not supported $\left(b_{3}=-.03, \beta_{3}=-.04, p=.33-\right.$ Table 8 , column 3$)$.

Yet, the effect size for this regression (equation 18) was 0.16 which is considered medium. This indicates that a moderate relationship exists between the predictor and demotivating gate experience - the outcome variable (Cohen 1988). 
Further, since the interaction term between politicized gate reviews and gate conditionality is not significant and gate conditionality does not have a significant main effect on demotivating gate experience, it can be concluded that gate conditionality is neither a moderator nor a predictor (Sharma, Durand and Gur-Arie 1981).

Overall, the greater the politicization of the gate reviews, the higher is the demotivation. However, the moderator, gate conditionality, has neither an association with demotivation nor any bearing on the association between politicization and demotivation.

\subsubsection{Test of Sub-Model 4}

Sub-model 4 examines the effect of a demotivating gate experience (Demot Gate Exp) on the innovativeness of firms' new products, and the moderating effect of market turbulence (Market Turb) and technological turbulence (Tech Turb). The three regression equations being compared are:

\footnotetext{
Innovativeness $=a+b_{1}$ Demot Gate Exp$$
\text { Innovativeness }=a+b_{1} \text { Demot Gate Exp }+b_{2} \text { Market Turb }+b_{3} \text { Tech Turb }
$$$$
\text { Innovativeness }=a+b_{1} \text { Demot Gate Exp }+b_{2} \text { Market Turb }+b_{3} \text { Tech Turb }
$$$$
+b_{4} \text { Demot Gate Exp X Market Turb }
$$$$
+b_{5} \text { Demot Gate Exp X Tech Turb }
$$ 
First innovativeness was regressed on demotivating gate experience (equation 21). This explained $5 \%$ of the variance in innovativeness $\left(F=7.5, p=.01\right.$, Adjusted $\left.R^{2}=.05\right)$. As Table 9 (column 1) shows, a demotivating gate experience had a significant negative relationship with innovativeness of firms' new products $\left(b_{1}=-.30, \beta_{1}=-.24, p=.00\right)$.

Table 9

Regression Results (Sub-Model 4)

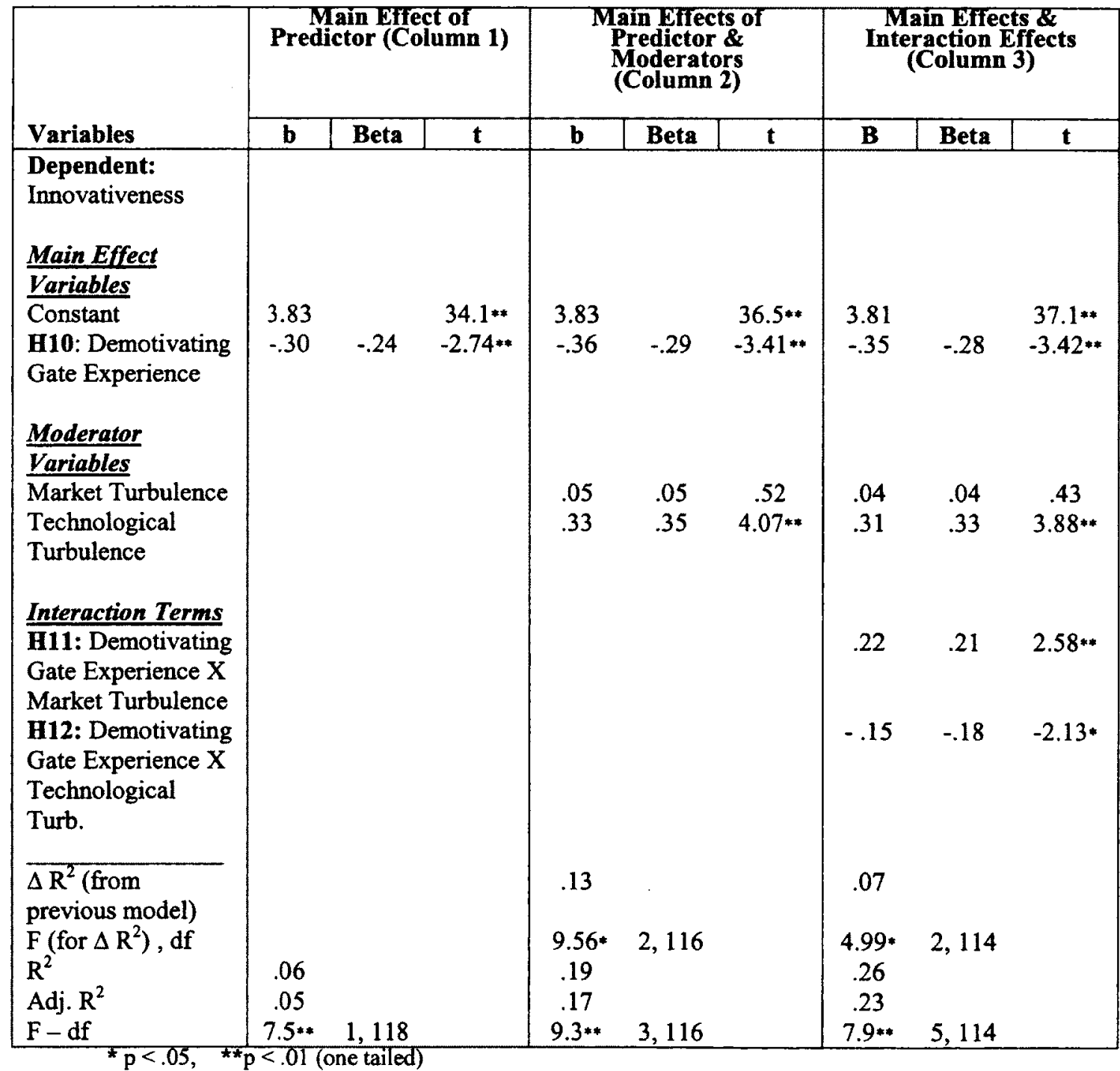


In the next regression, innovativeness was regressed on demotivating gate experience, market turbulence and technological turbulence (equation 22). This regression was significant and explained $17 \%$ of the variance in innovativeness $(F=9.3, p=.00$, Adjusted $\mathrm{R}^{2}=.17$ - Table 9, column 2). The change in $\mathrm{R}^{2}$ between equations 21 and 22 is .13. The F-test for the difference between the two models is significant $(F=9.56, \mathrm{df}=$ 2,$116 ; p<.05$ ), which indicates that the moderator variables are making a contribution to the model beyond the contribution made by demotivating gate experience in predicting innovativeness. However, market turbulence is not significantly related to innovativeness $\left(\mathrm{b}_{2}=.05, \beta_{2}=.05, \mathrm{p}=.30\right)$

In the third regression, innovativeness was regressed on demotivating gate experience, market turbulence, technological turbulence and the interaction between demotivating gate experience and market turbulence, as well as the interaction of demotivating gate experience with technological turbulence (equation 23). This regression was significant and explained $23 \%$ of the variance in innovativeness $\left(F=7.9, p=.00\right.$, Adjusted $R^{2}=.23$ Table 9, column 3). Variance inflation factors for independent variables in the model were also obtained and all were well below 10 , the value above which there should be concern for multicollinearity (Myers 1986). The F-test for the difference between the two models (equations 22 and 23 ) is significant $(F=4.99, \mathrm{df}=2,114 ; \mathrm{p}<.05)$. Thus the two interaction effects between demotivating gate experience and the moderators technological and market turbulence are making a significant contribution to the model over and above the contribution made by demotivating gate experience and technological turbulence in predicting innovativeness. 
As the above regression shows (Table 9, column 3), a demotivating gate experience reduces the innovativeness of firms' new products $\left(b_{1}=-.35, \beta_{1}=-.28, p=.00\right)$. Thus Hypothesis 10 is supported. Also, the interaction effects between demotivating gate experience and market turbulence $\left(b_{4}=.22, \beta_{4}=.21, p=.01\right)$ and between demotivating gate experience and technological turbulence $\left(b_{5}=-.15, \beta_{5}=-.18, p=.02\right)$ are significant, indicating they moderate the relationship between a demotivating gate experience and innovativeness. However, since $b_{4}$ has a positive sign, it implies that market turbulence tends to reduce the adverse effect of demotivating gate experience on innovativeness. This finding is contrary to what was hypothesized in $\mathrm{H} 11$ that market turbulence would worsen the main effect. Consistent with expectation, the moderating effect of technological turbulence $\left(b_{5}\right)$ has a negative sign implying that it worsens the negative effect of demotivating gate experience on innovativeness. Hypothesis 12 is thereby supported.

The effect size for this regression was 0.35 which is considered large. This suggests that a strong relationship exists between the predictors and the outcome variable innovativeness (Cohen 1988).

Further, since market turbulence does not have a significant main effect on innovativeness and the interaction between demotivating gate experience and market turbulence is significant (Table 9, column 3), it can be concluded that market turbulence is a pure moderator. Technological turbulence, on the other hand, is a quasi-moderator 
because it does have a significant main effect on innovativeness and its interaction with a demotivating gate experience is also significant (Sharma, Durand, and Gur-Arie 1981).

\subsubsection{Inflection Point for the Relationship between Demotivating Gate Experience and}

Innovativeness. To understand any changes in the nature of the association between demotivating gate experience (independent variable) and innovativeness (dependent variable) over the range of the moderator variables (market turbulence and technological turbulence), once again, the method suggested by Schoonhoven (1981) and used by Harris (2001) was employed. The inflection point at which the relationship between demotivating gate experience and innovativeness changes with change in the moderator variable needs to be identified. For this purpose, a partial derivative of equation 23 above was obtained with respect to demotivating gate experience and was set equal to zero. This yields:

$d$ Innovativeness / $d$ DemotGateExp $=b_{1}+b_{4}$ Market Turb $+b_{5}$ Tech Turb $=0$

Inflection Point for the Moderator Market Turbulence. This equation is first interpreted for the moderator market turbulence. To do so, the other moderator, technological turbulence is held at its mid- point because the variables in the regression have been mean centered (Aiken and West 1991; Jaccard, Turrisi, and Wan 1990). Since the data is mean centered, the mean of technological turbulence is zero. Therefore, equation 24 becomes: 
$\mathrm{d}$ Innovativeness / $\mathrm{d}$ Demot Gate Exp $=\mathrm{b}_{1}+\mathrm{b}_{4}$ Market Turb $=0$

Or, Market Turb $=-b_{1} / b_{4}$

Also, as suggested by Schoonhoven (1981) and Harris (2001) unstandardized coefficients were used in the equation. Substituting values of $b_{1}$ (coefficient of demotivating gate experience) and $b_{4}$ (coefficient of the interaction term between demotivating gate experience and market turbulence) in equation 26 , from the regression output (Table 9, column 3),

Market Turb $=-(-0.35) / 0.22=1.59$

This value of market turbulence represents the inflection point for the relationship between demotivating gate experience and innovativeness (Harris 2001; Schoonhoven 1981). In other words, when the value of mean-centered market turbulence is above 1.59 or non-mean-centered average values are above 5.78 (obtained by adding the mean of market turbulence 4.19 to the mean-centered value 1.59 ), the relationship between demotivating gate experience and innovativeness is positive and below this value the relationship is negative (when technological turbulence is at its mid-point).

Similar to the approach used in sub-model 1 , the above conclusion is based on equation 27 which in turn is based on equation 25 without equating it to zero. The values of the coefficients are included in equation 27. 
Two values of market turbulence were inserted in equation 27 , one at a time: +2.32 (which is +2 standard deviations from the mean) and -2.32 (which is -2 standard deviations). Market turbulence values of $+1-2$ standard deviations were chosen to generate two solutions to equation 27 , one that is above the inflection point and the other below it. With market turbulence equal to +2.32 (i.e. above the inflection point of 1.59) the value of equation 27 is +.16 and with market turbulence equal to -2.32 (i.e. below the inflection point of 1.59) the value of the equation is -.86 . Thus the relationship between demotivating gate experience and innovativeness is indeed positive above the inflection point and negative below it.

As market turbulence increases, the negative association between demotivation and innovativeness reduces. Before the inflection point, the relationship between demotivating gate experience and innovativeness is negative but steadily reduces with an increase in market turbulence. Beyond the inflection point, this relationship turns positive. Among the responding organizations, about $8.5 \%$ lie to the right of the inflection point, i.e., the range in which $d$ Innov/d Demot is positive ${ }^{6}$.

Inflection Point for the Moderator Technological Turbulence. Next, equation 24 is interpreted for the moderator technological turbulence, holding the other moderator

\footnotetext{
${ }^{6}$ The inflection point of +1.59 is +1.37 Std. Dev. from the mid-point of Market Turb (where Std. Dev is 1.16). Given that Market Turb data is near normal (as shown by the test of normality conducted earlier), from the normal table, $8.5 \%$ of the responding organizations lie to the right of the inflection point.
} 
market turbulence at its midpoint. Again, since the data is mean centered, the mean of market turbulence is zero. Therefore, equation 24 becomes:

$d$ Innovativeness / $d$ Demot Gate Exp $=b_{1}+b_{5}$ Tech Turb $=0$

Or, Tech Turb $=-b_{1} / b_{5}$

Substituting values of $b_{1}$ (coefficient of demotivating gate experience) and $b_{5}$ (coefficient of the interaction term between demotivating gate experience and technological turbulence) in equation 29 , from the regression output (Table 9, column 3),

Tech Turb $=-(-0.35 /(-0.15)=-2.33$

This value of Tech Turb represents the inflection point for the relationship between demotivating gate experience and innovativeness. When the value of mean-centered technological turbulence is above -2.33 or non-mean centered average value is above 2.31 (obtained by adding the mean of technological turbulence 4.64 to the mean-centered value -2.33 ), the relationship between demotivating gate experience and innovativeness is negative and below this value the relationship is positive (when market turbulence is at its mid-point). This conclusion is based on equation 30 below which in turn is based on equation 28 without equating it to zero. The values of the coefficients have been included in equation 30 .

d Innovativeness / d Demot Gate Exp $=-0.35+(-0.15)$ Tech Turb 
Two values of technological turbulence were inserted in this equation one at a time: +2.72 (which is +2 standard deviations from the mean) and -2.72 (which is -2 standard deviations). Technological turbulence values of $+/-2$ standard deviations were chosen to generate two solutions to equation 30 , one that is above the inflection point and the other below it. With technological turbulence equal to +2.72 (i.e. above the inflection point of -2.33) the value of equation 30 is -.76 and with technological turbulence equal to -2.72 (i.e. below the inflection point of -2.33 ) the value of the equation is +.06 . Thus the relationship between demotivating gate experience and innovativeness is indeed negative above the inflection point and positive below it.

As technological turbulence increases beyond the inflection point, the relationship between demotivating gate experience and innovativeness worsens. Among the responding organizations, about $95.6 \%$ lie to the right of the inflection point, i.e., the range in which $\mathrm{d}$ Innov/d Demot is in the negative range?

Overall, the analysis for Sub-model 4 shows that the higher the demotivation, the lower the innovativeness of new products. As market turbulence increases, the negative association between demotivation and innovativeness reduces. On the other hand, as technological turbulence increases, the negative association between demotivation and innovativeness worsens. The positive moderating effect of market turbulence and the negative moderating effect of technological turbulence are confirmed by the inflection point analyses. The inflection point analysis also suggests that a small fraction of

\footnotetext{
${ }^{7}$ The inflection point of -2.33 is -1.71 Std. Dev. from the mid-point of Tech Turb (where Std. Dev is 1.36). Given that Tech Turb data is near normal (as shown by the test of normality conducted earlier), from the normal table, $95.6 \%$ of the responding organizations lie to the right of the inflection point.
} 
responding organizations (about $8.5 \%$ ) lie in the range where an increase in market turbulence is associated with a positive relationship between demotivation and innovativeness. On the other hand, $95.6 \%$ of responding organizations lie in the range where an increase in technological turbulence is associated with a negative relationship between demotivation and innovativeness.

\subsubsection{Test of Sub-Model 5}

Sub-model 5 examines the effect of innovativeness on performance of new products. Thus, performance was regressed on innovativeness. Results show (Table 10) that the regression is significant and explained $18 \%$ of the variance in performance $(\mathrm{F}=26.3, \mathrm{p}$ $=.00$, Adjusted $\mathrm{R}^{2}=.18$ ). Innovativeness has a positive association with the performance of new products $\left(b_{1}=.36, \beta_{1}=.43, p=.00\right)$. Hypothesis 13 is supported.

The effect size was 0.22 (considered medium). This indicates a moderate relationship between the predictor and the outcome variable, performance (Cohen 1988).

Table 10

Regression Results (Sub-Model 5)

\begin{tabular}{|l|c|c|c|}
\hline \multirow{2}{*}{\multicolumn{1}{|c|}{ Variables }} & \multicolumn{3}{|c|}{ Main Effect of Predictor } \\
\cline { 2 - 4 } & B & Beta & T \\
\hline Dependent: Performance & & & \\
Main Effect Variables & & & \\
\hline Constant & 3.72 & & $42.2^{* *}$ \\
H13: Innovativeness & .36 & .43 & $5.13 * *$ \\
$\mathrm{R}^{2}$ & .18 & & \\
Adj. $\mathrm{R}^{2}$ & .18 & & \\
F-df & $26.3^{* *}$ & 1,118 \\
\hline \multicolumn{2}{|c|}{$\mathrm{p}<.05, \quad{ }^{* \star} \mathrm{p}<.01$ (one tailed) } \\
\hline
\end{tabular}




\subsection{Additional Analysis: Mediation}

The hypothesized framework of relationships has several concepts in a sequence, and many relationships appear to be mediated. Therefore, as additional analysis, tests of mediation were conducted.

Following Baron and Kenny (1986), the test of mediation includes the following steps. Let us say $\mathrm{X}$ is a predictor variable and $\mathrm{M}$ is the mediating variable through which $\mathrm{X}$ is believed to affect the outcome $\mathrm{Y}$. First, $\mathrm{Y}$ is regressed on the predictor $\mathrm{X}$ and the total effect is obtained (Step a). Second, M is regressed on X (Step b). Third, Y is regressed on $\mathrm{M}$ and $\mathrm{X}$ (Step $\mathrm{c}$ ). If the coefficient of $\mathrm{X}$ in step $\mathrm{a}$ is reduced in step $\mathrm{c}$ when $\mathrm{M}$ is in the model, then $\mathrm{M}$ partially mediates the $\mathrm{X}-\mathrm{Y}$ relationship. On the other hand, if the coefficient of $\mathrm{X}$ in step $\mathrm{c}$ is reduced to zero, then $\mathrm{M}$ completely mediates the $\mathrm{X}-\mathrm{Y}$ relationship.

$$
\begin{aligned}
& \text { Step } a-\cdots=b_{0}+b_{1} X \\
& \text { Step } b \text { - } M=b_{2}+b_{3} X \\
& \text { Step c - - } Y=b_{4}+b_{5} M+b_{6} X
\end{aligned}
$$

However, subsequently, researchers have modified the above steps and step a is no longer considered necessary (Kenny, Kashy, and Bolger 1998; MacKinnon, Fairchild, and Fritz 2007). In effect, even if there is no relationship between $X$ and $Y$ to begin with, if $M$ is related to $\mathrm{X}$ and $\mathrm{Y}$ (steps $\mathrm{b}$ and $\mathrm{c}$ ), $\mathrm{M}$ is considered to fully mediate the $\mathrm{X}-\mathrm{Y}$ relationship. 
Subsequent to this to establish mediation, it is advised that a test of indirect effect should be conducted to assess the significance of the indirect path ( $X$ to $M$ to $Y$ ), i.e., it should be checked that this path is not equal to zero (Preacher and Hayes 2004). This test is done using a bootstrapping approach developed by Preacher and Hayes ${ }^{8}(2004)$. In this test, a large number of samples of size $\mathrm{n}$ (where $\mathrm{n}$ is the original sample size) are taken from the data, sampling with replacement is done, and the indirect effect is computed in each sample. The average of the indirect effect is computed over the large number of bootstrapped samples, and a $95 \%$ confidence interval is constructed. If zero is not in the confidence interval, it can be concluded that the indirect effect is significantly different from zero, suggesting that the indirect path ( $\mathrm{X}$ to $\mathrm{M}$ to $\mathrm{Y}$ ) or mediation exists.

6.6.1 Test of the Mediating Effect of Power Accumulation. Following the above steps, first, it was examined whether a political organizational climate has a direct effect on the politicization of gate reviews or if it influences the politicization of gate reviews through power accumulation. In step a, politicization of gate reviews $(\mathrm{Y})$ was regressed on political organizational climate $(\mathrm{X})$. The results show (Table 11) that political organizational climate has a significant positive effect on politicization of gate reviews (b $=.39, \mathrm{p}=.00$ ). Then, from the regression results it can be seen that political organizational climate (X) has a significant and positive relationship with power accumulation (M) (b $=.31, \mathrm{p}=.00)($ step $\mathrm{b})$. In step $\mathrm{c}$, politicization of gate reviews $(\mathrm{Y})$ was regressed on power accumulation (M) and political organizational climate (X). Both $\mathrm{X}$ and $\mathrm{M}$ had a significant positive effect on $\mathrm{Y}$. With the inclusion of power accumulation in the model

\footnotetext{
${ }^{8}$ The script for conducting the test with SPSS was obtained from Preacher and Hayes' website (www. Comm.ohio-state.edu/ahayes/SPSS\%20programs/indirect.htm).
} 
in step $\mathrm{c}$, the coefficient for political organizational climate was slightly reduced $(b=.33$, $\mathrm{p}=.00)$ compared with its coefficient in step $\mathrm{a}(\mathrm{b}=.39, \mathrm{p}=.00)$. Thus, the effect of political organizational climate on politicization of gate reviews is partially mediated by power accumulation.

\section{Table 11}

\section{Test of Mediation (Mediator - Power Accumulation)}

\begin{tabular}{|c|c|c|c|c|c|c|c|c|c|}
\hline \multirow[b]{2}{*}{ Variables } & \multicolumn{3}{|c|}{$\begin{array}{c}\text { Efrect of Political } \\
\text { Organizational Climate } \\
\text { (X) on Power } \\
\text { Accumulation (M) } \\
\text { (Step b) }\end{array}$} & \multicolumn{3}{|c|}{$\begin{array}{l}\text { Efrect of Political } \\
\text { Organizational Climate } \\
\text { (X) On Politicization of } \\
\text { Gate Reviews (Y) } \\
\text { (Step a) }\end{array}$} & \multicolumn{3}{|c|}{$\begin{array}{l}\text { Effect of Political } \\
\text { Organizational Climate } \\
\text { (X) \& Power } \\
\text { Accumulation (M) on } \\
\text { Politicization of Gate } \\
\text { Reviews (Y) (Step c) }\end{array}$} \\
\hline & b & Beta & $t$ & b & Beta & $t$ & b & Beta & $t$ \\
\hline Constant & .00 & & & -.01 & & & -.01 & & \\
\hline $\begin{array}{l}\text { Political } \\
\text { Organizational } \\
\text { Climate }(\mathrm{X})\end{array}$ & .31 & .48 & $5.91^{* *}$ & .39 & .48 & $5.87 * *$ & .33 & .40 & $4.33^{* *}$ \\
\hline $\begin{array}{l}\text { Power } \\
\text { Accumulation } \\
(\mathrm{M})\end{array}$ & & & & & & & .21 & .17 & $1.83 *$ \\
\hline $\begin{array}{l}\mathrm{R}^{2} \\
\text { Adj. } \mathrm{R}^{2} \\
\mathrm{~F}-\mathrm{df}\end{array}$ & $\begin{array}{l}.23 \\
.22 \\
34.9 * *\end{array}$ & 1,118 & & $\begin{array}{l}.23 \\
.22 \\
34.5 * *\end{array}$ & 1,118 & & $\begin{array}{l}.25 \\
.24 \\
19.3 * *\end{array}$ & 2,117 & \\
\hline
\end{tabular}

Analyses for steps ' $a$ ' and ' $c$ ' have been shown next to each other to facilitate comparison

Further, to test if the indirect effect (Political Organizational Climate to Power

Accumulation to Politicization of Gate Reviews) is significantly different from zero, the test of indirect effects using the bootstrapping approach was conducted. Bootstrapping was done with 2000 samples. The $95 \%$ confidence interval so obtained ranged from 0.0035 to 0.1529 . Since zero does not appear in this range, it can be concluded that the 
indirect effect is significantly different from zero and mediation through Power Accumulation exists (Preacher and Hayes 2004).

6.6.2 Test of the Mediating Effect of Politicization of Gate Reviews. It was examined whether power accumulation $(\mathrm{X})$ has a direct effect on demotivating gate experience $(\mathrm{Y})$ or if its influence works through politicization of gate reviews (M). In step a, demotivating gate experience was regressed on power accumulation. The results showed (Table 12) that power accumulation has a positive effect on demotivating gate experience $(\mathrm{b}=.32, \mathrm{p}=.00)$. In other words, as power accumulation increases, product development managers are more demotivated.

In step $b$, politicization of gate reviews (M) was regressed on power accumulation (X). This regression was significant (Table 12) and power accumulation had a significant positive effect on the politicization of gate reviews $(b=.45, p=.00)$. In step $c$, demotivating gate experience $(\mathrm{Y})$ was regressed on both politicization of gate reviews (M) and power accumulation (X). This regression too was significant and both $\mathrm{X}$ and $\mathrm{M}$ had a significant positive effect on $\mathrm{Y}$. However, the coefficient for power accumulation was smaller in this step $(b=.21, p=.01)$ than in step $a(b=.32, p=.00)$, thereby indicating partial mediation. 
Table 12

Test of Mediation (Mediator - Politicization of Gate Reviews)

\begin{tabular}{|c|c|c|c|c|c|c|c|c|c|}
\hline \multirow[b]{2}{*}{ Variables } & \multicolumn{3}{|c|}{$\begin{array}{l}\text { Efrect of Power } \\
\text { Accumulation (X) on } \\
\text { Politiciciation of Gate } \\
\text { Reviews (M) (Step b) }\end{array}$} & \multicolumn{3}{|c|}{$\begin{array}{c}\text { Efrect of Power } \\
\text { Accumulation (X) On } \\
\text { Demotivating Gate } \\
\text { Experience (Y) } \\
\text { (Step a) }\end{array}$} & \multicolumn{3}{|c|}{$\begin{array}{c}\text { Effect of Power } \\
\text { Accumulation (X) \& } \\
\text { Politicization of Gate } \\
\text { Reviews (M) on } \\
\text { Demotivating Gate } \\
\text { Experience (Y) } \\
\text { (Step c) }\end{array}$} \\
\hline & $\mathbf{b}$ & Beta & $t$ & b & Beta & $t$ & b & Beta & $t$ \\
\hline Constant & -.01 & & & -.00 & & & .00 & & \\
\hline $\begin{array}{l}\text { Power } \\
\text { Accumulation } \\
\text { (X) }\end{array}$ & .45 & .36 & $4.14 * *$ & .32 & .31 & $3.55^{* *}$ & .21 & .20 & $2.26 *$ \\
\hline $\begin{array}{l}\text { Politicization of } \\
\text { Gate Reviews } \\
\text { (M) }\end{array}$ & & & & & & & .24 & .30 & $3.38^{* *}$ \\
\hline $\begin{array}{l}R^{2} \\
\text { Adj. } R^{2} \\
F-d f\end{array}$ & $\begin{array}{c}.13 \\
.12 \\
17.2^{* *} \\
\end{array}$ & 1,118 & & $\begin{array}{c}.10 \\
.09 \\
12.6^{* *} \\
\end{array}$ & 1,118 & & $\begin{array}{l}.18 \\
.16 \\
12.6 * *\end{array}$ & 2,117 & \\
\hline
\end{tabular}

Further to test if the indirect effect (power accumulation to politicization of gate reviews to demotivating gate experience) is significantly different from zero, the test of indirect effects using the bootstrapping approach was conducted. Bootstrapping was done with 2000 samples. The $95 \%$ confidence interval that was obtained ranged from 0.0399 to 0.2231. Since zero does not appear in this range, it can be concluded that the indirect effect is significantly different from zero and mediation through politicization of gate reviews exists (Preacher and Hayes 2004).

6.6.3 Test of the Mediating Effect of Demotivating Gate Experience. Furthermore, it was examined if politicization of gate reviews has a direct effect on the innovativeness of new products or if its influence on innovativeness works through demotivating gate 
experience. In step a, innovativeness $(\mathrm{Y})$ was regressed on politicization of gate reviews $(\mathrm{X})$. The regression model is not significant suggesting that the variable politicization of gate reviews has no direct effect on innovativeness (Table 13). In step b, demotivating gate experience $(M)$ was regressed on politicization of gate reviews $(X)$. This regression was significant and politicization of gate reviews was seen to lead to an increase in demotivating gate experience $(b=.30, p=.00)$. In step $\mathrm{c}$, innovativeness $(\mathrm{Y})$ was regressed on both demotivating gate experience $(\mathrm{M})$ and politicization of gate reviews (X). This regression was significant and demotivating gate experience (M) had a significant negative effect on innovativeness $(\mathrm{Y})$.

Even though in step a, politicization of gate reviews $(\mathrm{X})$ was found to have no direct effect on innovativeness $(Y)$, following the revised criteria for mediation suggested by Kenny, Kashy and Bolger (1998), it can be said that the politicization of gate reviews affects innovativeness only through demotivating gate experience, because other requirements (steps $b$ and c) for mediation have been established (i.e. politicization of gate reviews is related to demotivating gate experience, which in turn is related to innovativeness even with politicization of gate reviews in the model).

Further to test if the indirect effect (politicization of gate reviews to demotivating gate experience to innovativeness) is significantly different from zero, the test of indirect effects using bootstrapping was conducted. Bootstrapping was done with 2000 samples. The $95 \%$ confidence interval obtained ranged from -0.2139 to -0.0212 . Since zero does 
not appear in this range, it can be concluded that the indirect effect is significantly different from zero and mediation through demotivating gate experience exists.

Table 13

Test of Mediation (Mediator - Demotivating Gate Experience)

\begin{tabular}{|c|c|c|c|c|c|c|c|c|c|}
\hline \multirow[b]{2}{*}{ Variables } & \multicolumn{3}{|c|}{$\begin{array}{l}\text { Erfect of Politicization } \\
\text { of Gate Reviews (X) } \\
\text { on Demotivating Gate } \\
\text { Experience (M) (Step } \\
\text { b) }\end{array}$} & \multicolumn{3}{|c|}{$\begin{array}{l}\text { Effect of } \\
\text { Politicization of Gate } \\
\text { Reviews (X) on } \\
\text { Innovativeness (Y) } \\
\text { (Step a) }\end{array}$} & \multicolumn{3}{|c|}{$\begin{array}{c}\text { Effect of Politicization } \\
\text { of Gate Reviews }(X) \& \\
\text { Demotivating Gate } \\
\text { Experience (M) on } \\
\text { Innovativeness (Y) } \\
\text { (Step c) }\end{array}$} \\
\hline & b & Beta & $t$ & b & Beta & $t$ & b & Beta & $t$ \\
\hline Constant & .00 & & & -.00 & & & -.00 & & \\
\hline $\begin{array}{l}\text { Politicization of } \\
\text { Gate Reviews } \\
\text { (X) }\end{array}$ & .30 & .38 & $4.40 * *$ & -.11 & -.11 & -1.22 & -.02 & -.02 & -.24 \\
\hline $\begin{array}{l}\text { Demotivating } \\
\text { Gate Experience } \\
\text { (M) }\end{array}$ & & & & & & & -.29 & -.24 & $-2.44 * *$ \\
\hline $\begin{array}{l}R^{2} \\
\text { Adj. } R^{2} \\
F-d f\end{array}$ & $\begin{array}{l}.14 \\
.13 \\
19.3 * *\end{array}$ & 1,118 & & $\begin{array}{l}.01 \\
.00 \\
1.5\end{array}$ & 1,118 & & $\begin{array}{r}.06 \\
.04 \\
3.7 *\end{array}$ & 2,117 & \\
\hline
\end{tabular}

6.6.4 Test of the Mediating Effect of Innovativeness. Finally, it was examined if a demotivating gate experience has a direct effect on performance or if its effect works through innovativeness. In step a, performance $(\mathrm{Y})$ was regressed on demotivating gate experience (X). The regression is significant (Table 14) and demotivating gate experience has a negative effect on performance $(b=-.21, p=.01)$. In step $b$, innovativeness $(M)$ was regressed on demotivating gate experience $(X)$. This regression was also significant and demotivating gate experience has a negative effect on innovativeness $(b=-.30, p=.00)$. In step $c$, performance $(Y)$ was regressed on both innovativeness $(\mathrm{M})$ and demotivating gate experience $(\mathrm{X})$. This regression was 
significant but while innovativeness had a positive effect on performance $(b=.34, p$ $=.00$ ), the effect of demotivating gate experience on performance was not significant (b $=-11, \mathrm{p}=.12$ ). So with the introduction of the mediator innovativeness in the third regression, the effect of demotivating gate experience $(\mathrm{X})$ vanished, thereby indicating that innovativeness completely mediates the relationship between demotivating gate experience and performance.

Table 14

Test of Mediation (Mediator - Innovativeness)

\begin{tabular}{|c|c|c|c|c|c|c|c|c|c|}
\hline \multirow[b]{2}{*}{ Variables } & \multicolumn{3}{|c|}{$\begin{array}{l}\text { Effect of } \\
\text { Demotivating Gate } \\
\text { Experience (X) on } \\
\text { Innovativenss (M) } \\
\text { (Step b) }\end{array}$} & \multicolumn{3}{|c|}{$\begin{array}{l}\text { Efrect of } \\
\text { Demotivating Gate } \\
\text { Experience }(X) \text { on } \\
\text { Performance }(Y) \\
\text { (Step a) }\end{array}$} & \multicolumn{3}{|c|}{$\begin{array}{c}\text { Effect of Demotivating } \\
\text { Gate Experience (X) } \\
\text { \& Innovativeness (M) } \\
\text { on Performance (Y) } \\
\text { (Step c) }\end{array}$} \\
\hline & b & Beta & $t$ & b & Beta & $\bar{t}$ & b & Beta & $\bar{t}$ \\
\hline Constant & -.00 & & & .00 & & & .00 & & \\
\hline $\begin{array}{l}\text { Demotivating } \\
\text { Gate Experience } \\
\text { (X) }\end{array}$ & -.30 & -.24 & $-2.74 * *$ & -.21 & -.20 & -2.22 & -.11 & -.10 & -1.20 \\
\hline $\begin{array}{l}\text { Innovativeness } \\
\text { (M) }\end{array}$ & & & & & & & .34 & .40 & $4.69 * *$ \\
\hline $\begin{array}{l}R^{2} \\
\text { Adj. } R^{2} \\
F-d f\end{array}$ & $\begin{array}{l}.06 \\
.05 \\
7.5 * * \\
\end{array}$ & 1,118 & & $\begin{array}{r}.04 \\
.03 \\
5.0 * \\
\end{array}$ & 1,118 & & $\begin{array}{l}.19 \\
.18 \\
13.9 * * \\
\end{array}$ & 2,117 & \\
\hline
\end{tabular}

Further to test if the indirect effect (demotivating gate experience to innovativeness to performance) is significantly different from zero, the test of indirect effects using bootstrapping was conducted. Bootstrapping was done with 2000 samples. The $95 \%$ confidence interval that was obtained ranged from -0.2206 to -0.0198 . Since zero does not appear in this range, it can be concluded that the indirect effect is significantly different from zero and mediation through innovativeness exists. 


\subsection{Summary of Results}

The results of the data analysis support most of the relationships proposed in the hypotheses. The relationships found suggest that a political organizational climate leads senior managers affected by new product development to accumulate more power, and this tendency is enhanced when the organization has more slack and a high degree of centralization. Also, power accumulation by senior managers makes the gate reviews more politicized. Strict gate review criteria, objective criteria and an entrepreneurial/innovative culture do not affect the extent to which power accumulation influences politicization of gate reviews. Furthermore, when gate reviews become politicized, they create a demotivating gate experience for product development managers who take their products through gate reviews. Even if gate review criteria are conditional in nature, it does not change the extent to which the politicization of gate reviews makes the gate experience demotivating. Additionally, results indicate that when the experience of gate reviews is demotivating, the innovativeness of the firm's new products is reduced. The adverse effect of demotivating gate experience on innovativeness is reduced by market turbulence. On the other hand, the adverse influence of demotivating gate experience on innovativeness is worsened by technological turbulence. Finally, innovativeness and performance are positively related. In other words, when innovativeness is low because of the influence of power and politics, the performance of new products is adversely affected. 
In terms of mediation, power accumulation partially mediates the effect of political organizational climate on politicization of gate reviews. Similarly, politicization of gate reviews partially mediates the influence of power accumulation on demotivating gate experience. On the other hand, demotivating gate experience fully mediates the effect of politicization of gate reviews on innovativeness. Similarly, innovativeness fully mediates the effect of demotivating gate experience on performance. In effect, all the mediating effects for the variables in the research framework exist either partially or fully. 
Figure 2

Results of Hypothesis Testing

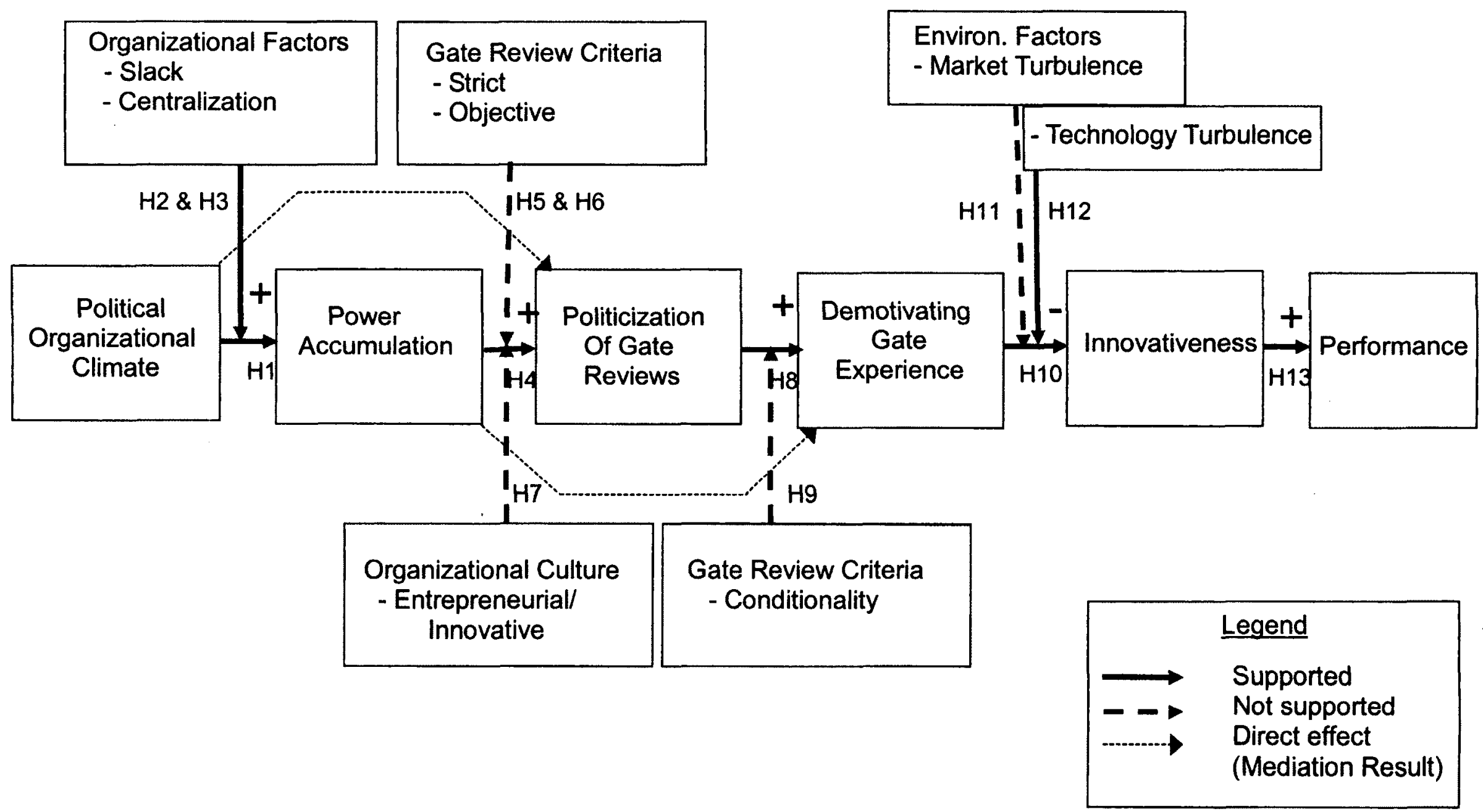




\section{QUALITATIVE RESEARCH METHOD, DATA ANALYSIS, \& RESULTS}

The purpose of qualitative research was to understand if the organizational reality is consistent with the conceptualization developed here based on the literature and is consistent with the quantitative study results. Therefore, qualitative research adopted a post-positivistic approach to qualitative research framed by quantitative research, using depth interviews with managers in the field (Opsina 2004). The approach was designed to ground interpretation of the quantitative findings in the personal experiences and perspectives of the managers. The open-ended nature of the interview process allows managers, like those who responded to the quantitative study, to elaborate more fully on their experiences and their interpretations of these experiences in a more holistic fashion. The interview protocol structure framed the elements of this interpretation that were supportive of further elaboration on the key concepts and relationships studied. The more open-ended nature of the questions allowed for answers which were less predictable and unexpected and at the same time, more culturally rich and salient in meaning to the respondents in their lived experience and their interpretation of these experiences (Opsina 2004).

Specifically, the qualitative research has three objectives:

1. to generate a better understanding of how the relationships modeled in this research actually occur in organizations.

2. to understand why some of the hypothesized relationships have not been confirmed in the quantitative data analysis phase. 
3. to generate some new insights regarding the broad phenomena being studied in this dissertation. These new insights may provide directions for future exploration.

\subsection{Qualitative Research Method}

Based on the findings of the quantitative survey, a uniform protocol containing semistructured questions was developed and used for the interviews (Appendix 4). The questions were formulated around each of the hypothesized paths of the model to confirm the findings of supported hypotheses. Where hypothesized relationships were not supported, questions were formulated to explore the original hypothesis and to further explore the nature and basis of the reasons for not finding support for the hypotheses if the managers agreed that the proposed relationships were not supportable. The semistructured format left scope for new insights to emerge during the interviews, which were probed further (McCracken 1988; Myers 2009).

Having an interview protocol ensured that all the terrain was covered in the same order for each respondent, while still maintaining the conversational context of each interview (McCracken 1988). Further, prompts were carefully created and placed in the protocol, since in the demanding circumstances of the interview it is usually difficult to formulate or recall such prompts. The protocol also established the direction and scope of the interview. Further, the protocol allowed attention to be focused on what the respondent was saying without being distracted by what to ask next (McCracken 1988). 
The semi-structured nature of the protocol and questions provided the opportunity to explore further and seek unstructured responses. Any such opportunity that presented itself in an interview was to be pursued to increase understanding of issues and reveal relationships or insights that had not been considered earlier (McCracken 1988). Questions in the protocol allowed the respondents to talk on their own about their experiences with gate reviews (McCracken 1988).

The protocol was revised several times in consultation with the dissertation supervisor. Prompts and skip patterns were developed to ensure that the interview process would flow smoothly without missing appropriate questions or overburdening respondents with unnecessary questions unsuited to their earlier responses. The protocol was then pretested with three product development managers to ensure that the questions were clear and there was no ambiguity. The feedback from the pre-test helped further refine the protocol and suggested additional probes that would lead to better understanding of the issues involved.

Once the protocol was finalized, interviews were conducted with product development managers from a variety of organizations. Based on earlier published qualitative studies on new product development, it was felt that data from around 12 organizations would be sufficient to generate meaningful insights (Danneels 2002; Dougherty and Hardy 1996; Dougherty and Heller 2004). Furthermore, it was planned that data collection (or interviews) would stop when redundancy with regard to information was achieved. 
The following criteria were kept in view while selecting organizations and the responding managers for qualitative data collection purposes. The organizations should be from a diverse range of industries. In terms of the size of the organization, there should be a mix of medium and large enterprises that normally use the Stage-Gate process. The organizations should be actively involved in new product development and should have the Stage-Gate process or some variant of it in place. The responding managers should have had the experience of navigating their products through the Stage-Gate or similar process. Finally, the responding managers should be at least at the middle-level or higher in the organizational hierarchy so that they would be aware of political pulls and pushes in their organizations.

Interviews were conducted with representatives from 12 organizations. A review of the interview responses indicated that the number of organizations used was appropriate as the information from later interviews supported earlier findings and entirely new information did not emerge (i.e., redundancy of information was setting in). Hence, it was decided to stop further interviews.

The 12 organizations represent a diverse range of industries such as industrial automation, electronics hardware, sports apparel, components for the automotive industry, and water filtration systems. Eight of them are large enterprises and the other four are medium. All are actively involved in new product development and use the 
Stage-Gate process or some variant of it ${ }^{9}$. The organizations vary in terms of their innovation focus (from 3 to 6 on a 1 to 7 scale where $1=$ not at all and $7=$ a great deal). This assessment of innovation focus is similar in spirit to the measurement of innovative culture in the quantitative study. The number of review gates in their Stage-Gate processes vary from 3 to 6 (details in Appendix 6).

As with the survey participants, all the managers participating in the qualitative interviews have worked on new product development projects and have the experience of navigating their products through the Stage-Gate process. They are at least mid-level or higher managers.

The managers were assured that their identity and that of their organization would be kept completely anonymous. Hence, instead of the actual names of the organizations, pseudonyms are being used below in the reports of the results.

To begin with, the respondents were provided a brief overview of the area of research (Appendix 4). Then, they were asked questions that had been developed based on the relationships being captured in different sub-models of the conceptual model. Specifically, they were requested to explain whether or not the relationships posited in the hypotheses in the quantitative part of the study exist in their organizations. Next, they were asked to comment on the hypothesized effects that were contradicted by the results of quantitative analysis. In addition, if their own experiences were not consistent with the

\footnotetext{
${ }^{9}$ These managers were selected from organizations that have contact/relationship with Clarkson University School of Business, Potsdam, New York and from members of the Western New York Chapter of the Product Development Management Association (PDMA).
} 
quantitative results or the hypothesized effects, they were asked to explain the inconsistencies. If they provided an observation or an explanation that was different from the assumptions underlying the conceptual model, they were asked to explain the observation in detail. Such explanations provided new insights.

Interviews lasted from one hour to one hour forty five minutes. All the issues were carefully discussed to develop a clear understanding of respondents' experiences. After the managers explained their experiences, their responses on the key issues were repeated back to them to ensure that their experiences were accurately understood. Detailed notes were taken and a summary prepared for each interview (See Appendices 5a to 51). The summaries are presented in the appendices, arranged in order from the organization with the most political climate (Appendix 5a) to the least (Appendix 51). Six organizations have a highly political climate (Appendices $5 \mathrm{a}$ to $5 \mathrm{f}$ ) and two of them have a moderately political climate (Appendices $5 \mathrm{~g}$ and $5 \mathrm{~h}$ ). In the remaining four, the level of politics is low (Appendices 5i to 51).

\subsection{Findings of Qualitative Research}

The interview notes were compared and contrasted to look for common and unique themes related to the relationships modeled in this dissertation, the explanations for the unconfirmed effects, and the new insights related to the broad phenomena under study. 
Overall, the interviews provided good support for the conceptual model except for one of the moderating effects (i.e., of technological turbulence) which was moderately supported. How the interviews offered support for the model and explanations for the unconfirmed effects is discussed below in the context of each of the sub-models of the study. Thereafter, new insights generated across all the interviews and sub-models are presented.

\subsubsection{Findings for Sub-Model 1}

\subsubsection{Effect of Political Organizational Climate on Power Accumulation. Interview data} from all 12 organizations suggest that a political organizational climate is related to power accumulation in the hypothesized direction, which is consistent with the quantitative results (See Appendix 7).

In the case of companies where the organizational climate was highly political, power accumulation was also high (Appendices 5a to $5 \mathrm{f}$ ). As the respondent from FABRICO (Appendix 5e) remarked, "The climate in our organization is quite political and senior managers try to become powerful by controlling more physical and human resources, whenever they get the opportunity."

On the other hand, in organizations where politics was low, as expected, power accumulation was also low (Appendices $5 \mathrm{i}$ to 51 ). In the remaining moderately political organizations (Appendices $5 \mathrm{~g}$ and $5 \mathrm{~h}$ ), as expected, power accumulation was moderate. 
The organizations that have low levels of politics and power accumulation have varied reasons for being in that state. The respondent from HARSOCO (Appendix 51) said "We have been under severe threat, sales have fallen, and it seemed at one point that we might have to close down. Thousands of employees have been laid off and for those who have remained it is a question of survival. Everyone knows that this is not the time to play politics; instead, we need to pull together and do our best so that the firm can come out of the problems it has been facing and become a strong player in the industry again." In effect, this threat to survival reduces the extent of politics and through it, power accumulation.

The respondent from INDAUTCO (Appendix 5i) explained, "We are a medium-size company that is privately owned by two engineers, and both of them are closely involved in the operations of the company including product development. As a result, senior managers focus on what is best for the organization and not on anyone's personal or political interests."

APPCO (Appendix 5k) is a medium-size (150 employees) close-knit organization which only designs and markets products and fully outsources its manufacturing. The organization is highly dedicated to excelling in the market. There is a high degree of connectedness across departments and across management levels, which leaves little scope for politics and power acquisition to surface. 
ELECTCO's (Appendix 5j) R\&D division has historically had low politics and power acquisition by senior managers. This is attributed to the present and past division chiefs who, through their leadership style and close involvement in the operations of the division, ensured that politics and power plays are kept to a minimum.

In sum, a majority of the organizations in the sample have a moderate to highly political climate and thus moderate to high power accumulation by senior managers. The interesting fact is that in the remaining four organizations, managerial actions minimize the level of politics. For example, politics is low because one of the organizations is highly connected and market focused; in another, the owners/top managers are involved in new product development and reviews; and in yet another, the current and previous R\&D chiefs have been directly involved in the innovation process. In the fourth organization, politics is low because it happens to be in an environment where it faces a threat to its survival and its top managers seem to have leveraged that threat to motivate employees to focus intensely on innovation and forsake their personal interests.

\subsubsection{Moderating Effect of Centralization on the Political Organizational Climate -}

Power Accumulation Relationship. A majority of the responses (11 out of 12) suggest that centralization influences the relationship between political organizational climate and power accumulation in the hypothesized direction, which is consistent with the quantitative findings (Appendix 7). 
In the case of organizations in which the climate is more political (COMPCO - 5a, MACHCO - 5b, FILTCO - 5c, ELECMACO - 5d, FABRICO - 5e, SOFTCO - 5f), a high or moderate level of centralization leads to an increase in power accumulation. The respondent from FABRICO (Appendix 5e) explained this effect by stating that when centralization is high in a political organization, power accumulation is senior managers' way of gaining influence. The respondent from ELECMACO (Appendix 5d) offered a nuanced view of how centralization works in his organization. He said that there is a range of seniority among senior managers in the organization. Some of them are really top-level managers, while others are more toward the middle-level of seniority. While there is more power accumulation in a political climate when the organization is more centralized, it is the managers in the middle-level who try to acquire more power because they are driven to a large extent by their sense of job insecurity. In effect, it is not the very senior managers, who are generally quite secure in their positions, but the middlelevel managers under them who try more to gather power, in the case of centralization.

On the other hand, in organizations where the climate is less political (ELECTCO - 5j, APPCO - 5k, HARSOCO - 51), as expected, low levels of centralization do not lead to any increase in power accumulation. Similarly, in organizations with a moderate level of politics (SEMCO - 5g and COMAPPCO - 5h), low levels of centralization do not enhance power accumulation.

The only exception among the twelve organizations is INDAUTCO (Appendix 5i) where centralization is high, but it does not influence power accumulation because the level of 
politics and power accumulation is low to begin with. As mentioned earlier, INDAUTCO is a medium-size, privately held company in which the owners are actively involved in the operations and new product development - which likely further prevents centralization from increasing power accumulation.

In sum, when the organization is political, centralization makes senior managers acquire more power because this is their way of gaining influence. On the other hand, when the climate is less political, this urge to gain influence is low; thus, whether centralization is high or low, senior managers are not driven to acquire power.

\subsubsection{Moderating Effect of Slack on the Political Organizational Climate - Power} Accumulation Relationship. Data from two-thirds of the organizations (8 out of 12) suggests that slack influences the relationship between political organizational climate and power accumulation in the hypothesized direction, which is consistent with the results of quantitative analysis (Appendix 7).

In the case of organizations in which the climate is more political, a high level of slack (MACHCO - 5b, SOFTCO - 5f) increases power accumulation. The one exception where politics is high but high slack does not influence power accumulation is ELECMACO (Appendix 5d). As discussed earlier, in this organization, power is acquired mainly by managers who are not very senior, but belong to the middle-level of seniority. The respondent from ELECMACO explained, "When the organization is doing well and is profitable and there are surplus resources available, the middle-level 
managers feel less insecure about their jobs and thus try less to acquire power." In other words, high slack does not influence the political climate-power accumulation relationship in this organization in the manner suggested by the conceptual model. In addition, in three organizations in which the climate is highly political (COMPCO - 5a, FILTCO $-5 c$, FABRICO - 5e), a low level of slack, as expected, does not lead to increased power accumulation.

Then, in the organization where the climate is less political and slack is low (HARSOCO $-51)$, consistent with the expectation, there is no increase in power accumulation. The three exceptions in this category (of low politics), where slack is high but does not play a role in influencing power accumulation are INDAUTCO (Appendix 5i), ELECTCO (Appendix 5j), and APPCO (Appendix 5k). This happens because in these three organizations, politics and power accumulation are low. The respondent from APPCO (Appendix 5k) explained this point by stating that since in her organization the level of politics is low, the basic drive to acquire surplus resources and become powerful is low on the part of senior managers.

In the case of organizations with moderate levels of politics (SEMCO $-5 \mathrm{~g}$ and COMAPPCO - 5h), low levels of slack, as expected, did not lead to increase in power accumulation. When asked to explain why, in their organizations, slack and centralization do not play any role in influencing power accumulation in a moderately political climate, the respondent from SEMCO (Appendix 5g) said, "The tendency to accumulate power comes from seeing politics everywhere in the firm and slack and 
centralization shouldn't make any difference." Similarly, the respondent from COMAPPCO (Appendix 5h) explained, "Slack and centralization do not make much difference because my organization is not so political and the desire to gather power is not so high."

In sum, in many of the organizations high slack leads to an increase in power accumulation, when politics is high. Similar to the effect of centralization, when the climate is less political, high slack does not lead managers to acquire more power because the basic drive to acquire surplus resources is low on the part of senior managers.

\subsubsection{Findings for Sub-Model 2}

\subsubsection{Effect of Power Accumulation on Politicization of Gate Reviews. Data from a} majority of organizations (11 out of 12) suggests that power accumulation is related to politicization of gate reviews in the hypothesized direction, which is consistent with the quantitative findings (Appendix 8).

In organizations where the climate is highly political, as discussed earlier, power accumulation is also high (COMPCO - 5a, MACHCO - 5b, FILTCO - 5c, ELECMACO $-5 \mathrm{~d}$, FABRICO $-5 \mathrm{e}$, and SOFTCO $-5 \mathrm{f}$ ). This high power accumulation in turn leads to increased politicization of gate reviews. The respondent from COMPCO (Appendix 5a) explained this point by stating, "Senior managers see gate reviews as an opportunity to make their case and achieve their political ends. They may support a new product only if 
it is put under their control and made in their manufacturing facility," i.e. if it enhances their power.

On the other hand, in organizations where the climate is less political, as discussed earlier, power accumulation is also low. As expected, this low level of power does not increase politicization of gate reviews (INDAUTCO - 5i, ELECTCO - 5j, APPCO - 5k, and HARSOCO - 51). While explaining why there are no serious attempts by senior managers to influence gate reviews, the respondent from INDAUTCO (Appendix 5i) said that since senior managers do not try to acquire power, they don't attempt to influence gate reviews. As mentioned earlier, one of the owners (in this privately held company) is involved in gate reviews, which ensures that projects are primarily reviewed on their merit.

The respondent from ELECTCO (Appendix 5j) explained, "The division develops products in response to customer requirements. So the product specifications and the development schedule are determined as per customer needs. This leaves little scope for senior managers to ask for changes in the project and reduces the opportunity to play politics."

In the organization where the climate is moderately political, as discussed earlier, power accumulation is also moderate (SEMCO $-5 \mathrm{~g}$ ). This moderate level of power accumulation, as expected, leads to moderate politicization of gate reviews. 
The only exception among these 12 organizations, where the politicization of gate reviews is low despite a moderate level of power accumulation is COMAPPCO (Appendix 5h). In this organization, a majority of projects involve only product upgrades and modifications, and there are very few new products. Senior managers don't see control over such projects bringing them any political benefit. So they tend not to influence project reviews in their favour.

Generally, it appears that powerful managers see gate reviews as an opportunity to achieve their political ends and in the process politicize them. However, there can be some exceptions, for example, when projects are only simple upgrades and modifications to existing products and senior managers do not see any political benefit in controlling them. Similarly, when project specifications are dictated by customer needs, it leaves little scope for senior managers to ask for changes and interfere for political reasons. On the other hand, as expected, when power accumulation is low (mostly driven by low organizational politics), the urge to politicize gate reviews is low on the part of senior managers.

\subsubsection{Moderating Effect of Strict and Objective Criteria on the Power Accumulation-} Politicization of Gate Reviews Relationship. Based on the Stage-Gate literature it was hypothesized that strict and objective criteria would reduce the adverse effect of power accumulation on the politicization of gate reviews. However, the results of quantitative analysis do not support the hypotheses. Interview data from all 12 organizations is 
consistent with the results of quantitative analysis, and about half of them ( 7 out of 12) could be interpreted as supporting the hypotheses ${ }^{10}$ (Appendix 8 ).

In organizations with high power accumulation in which criteria strictness and objectivity is moderate or high (COMPCO $-5 \mathrm{a}$, and SOFTCO $-5 \mathrm{f}$ ), consistent with the quantitative results but not with the hypotheses, strictness and objectivity do not reduce the adverse effect of power accumulation on politicization of reviews. When asked to explain why strictness and objectivity do not reduce politicization of gate reviews despite moderate or high power accumulation, the respondents generally said that if senior managers want to create problems for a project, they can always find a way to do so. Also, if they want to get a project through, they will, despite strict and objective criteria. For example, the respondent from SOFTCO (Appendix - 5f) explained, "If senior managers are really against a project, they somehow find a way to create problems for it and vice versa," i.e., if they are in favour, they get the project through - though he felt they don't do so very often. A respondent from MACHCO (Appendix $5 b$ ) felt that even if his organization were to have more strict and objective review criteria, it would reduce politicization of gate reviews only to a limited extent. According to him, "Powerful managers are influential and their interference finds its way into gate reviews despite strict and objective criteria."

When the organizations with high power accumulation have low criteria strictness and objectivity (MACHCO - 5b, FILTCO - 5c, ELECMACO - 5d, FABRICO - 5e), such

\footnotetext{
${ }^{10}$ Since the ratings on strictness and objectivity of criteria are the same for each organization and their effects are also the same, the discussion on their moderating effects has been combined and reported together in Table 3.
} 
lax, subjective criteria do not reduce the adverse effect of power accumulation, which is consistent with the results of quantitative analysis and with the hypothesized moderating effects.

In organizations with low power accumulation, the strictness and objectivity of criteria, whether high or moderate, have little effect on politicization of reviews because both power accumulation and politicization are low (INDAUTCO $-5 \mathrm{i}$, ELECTCO $-5 \mathrm{j})$. This is consistent with the quantitative findings but contrary to the hypotheses. Also, when organizations with low power accumulation have low criteria strictness and objectivity (APPCO - 5k, HARSOCO - 51), any adverse effect of power accumulation is not reduced, which is consistent with the quantitative results and the hypothesized moderating effects.

When asked why senior managers do not attempt to influence gate reviews despite low criteria strictness and objectivity, the respondent from HARSOCO (Appendix 51) explained, "Since senior managers don't attempt to influence gate reviews, they don't feel the need to exploit less rigorous criteria to influence gate review outcomes in their favour." Note that power accumulation is low in HARSOCO.

In the case of the organization with a moderate level of power accumulation and highly strict and objective criteria (COMAPPCO $-5 \mathrm{~h}$ ), consistent with the quantitative results but not with the hypotheses, strictness and objectivity do not reduce the adverse effect of power accumulation. When strictness and objectivity of criteria are low in the 
organization with a moderate level of power accumulation (SEMCO - 5g), such criteria do not reduce the adverse effect on politicization of reviews, which is consistent with quantitative results and with the moderating hypotheses. The respondent from SEMCO (Appendix 5g) explained, "If senior managers want to find fault with a project in order to scuttle it, they can find ways to do so. They can cast doubt on your ROI figures, that they are unrealistic and cannot be achieved."

In sum, there is strong consistency between the interview responses and the quantitative results. Several respondents explained this by saying that if senior managers want to create problems for a project, they can always find a way to do so, despite strict and objective criteria, and if they favour a project, they can get it through.

\subsubsection{Moderating Effect of Entrepreneurial/Innovative Culture on Power Accumulation}

- Politicization of Gate Reviews Relationship. It was hypothesized that an entrepreneurial/ innovative culture would weaken the adverse effect of power accumulation on the politicization of gate reviews. However, the quantitative results do not support this hypothesis.

Interview data from most organizations (11 out of 12) is consistent with the results of the quantitative analysis, and only about half of them (5 out of 12) could be interpreted as supporting the hypothesized moderating effect (Appendix 8). Since the ratings on entrepreneurial and innovative foci are very similar for each organization (correlation = 
0.97) and their effects are also identical, the discussion on their moderating effects has been combined and reported together in Appendix 8.

In organizations with high power accumulation and in which the culture is highly entrepreneurial/innovative (COMPCO - 5a, ELECMACO - 5d), consistent with the quantitative results but not with the hypothesis, an entrepreneurial/ innovative culture does not reduce the adverse effect of power accumulation on politicization of reviews. Further, in organizations with high power accumulation, when the culture is less entrepreneurial/ innovative (MACHCO - 5b, FILTCO - 5c, FABRICO - 5e) as expected, such a culture does not influence the adverse effect of power accumulation, which is consistent with the results of quantitative analysis and the hypothesized moderating effect.

In organizations with low power accumulation and a culture that is highly entrepreneurial/ innovative (INDAUTCO - 5i, ELECTCO - 5j, APPCO - 5k, HARSOCO - 5l), contrary to the hypothesis but consistent with the quantitative findings, such a culture does not reduce politicization of reviews. Similarly, in the case of the organization with a moderate level of power accumulation and a highly entrepreneurial/innovative culture (SEMCO $-5 \mathrm{~g}$ ), the culture does not reduce the adverse effect of power accumulation. This is consistent with the quantitative results but not with the hypothesized moderating effect. When the organizational culture is less entrepreneurial/innovative, with a moderate level of power accumulation (COMAPPCO - 
5h), the adverse effect on politicization of reviews is not reduced, which is consistent with the quantitative results and with the moderating hypothesis.

When asked to explain why an entrepreneurial/innovative culture does not reduce the politicization of gate reviews that follows from moderate or high power accumulation, the almost consistent response was that the interests of powerful managers are so strong that they are not easily deterred by an entrepreneurial/innovative culture. The respondent from FABRICO (Appendix 5e) summed it all up with, "Senior managers have their own agendas that they want to pursue and strict and objective criteria and an innovative culture is ignored in this pursuit. Senior managers can always find problems with a project and couch their political intentions in objective terms to create problems for the project they do not want to support."

The only exception out of the 12 organizations is SOFTCO (Appendix 5f). The respondent said that their strong innovative culture prevents gate reviews from getting politicized even though some senior managers are quite powerful. When asked to explain the inconsistency of his response with the quantitative findings, the respondent said, "In our case, it is not just our organization's culture but the entire industry is strongly focused on innovation, and that probably makes some difference." In other words, an innovation focus at the industry level may be a stronger force than the organization's own innovative culture in preventing powerful senior managers from politicizing gate reviews. 
On the whole, there is strong consistency between the interview responses and the quantitative findings about the moderating effect of entrepreneurial/innovative culture. Several respondents explained the absence of a moderating effect of an entrepreneurial/innovative culture by stating that senior managers are usually driven to pursue their own agendas and an entrepreneurial/innovative culture is ignored in this pursuit.

\subsubsection{Findings for Sub-Model 3}

\subsubsection{Effect of Politicization of Gate Reviews on Demotivation of Product Development}

Managers. Interview data from all 12 organizations suggests that politicization of gate reviews has a demotivating effect on product development managers, which is consistent with the quantitative findings and the hypothesis (Appendix 9).

The reasons provided in support of this relationship are nuanced and full of richness. For example, the respondent from ELECMACO (Appendix 5d) said, "The extent of demotivation among product development team members varies depending on their background. The engineers and technical experts are usually less demotivated than managers from non-technical backgrounds. If a project runs into problems at gate reviews or even gets cancelled, technical managers view it as a learning experience and feel that they can use the knowledge gained here in another project. It is the nontechnical people who get more demotivated when there is a setback on a project during the reviews." 
The respondent from SEMCO (Appendix 5g) while suggesting that a moderate level of politicization demótivates moderately, provided a nuanced explanation, "For some experienced product development managers though, this demotivation may not last long because they accept political meddling as a part of organizational reality. But, if their projects are repeatedly rejected, even these managers can get demotivated."

Similarly, the respondent from FABRICO (Appendix 5e) added, "If the setback to the project is not severe or fatal, product development teams recover their motivation quickly because they are experienced enough to know that in organizations there will always be politics and setbacks. If there is a more serious obstacle to the project, then demotivation can last longer." The respondent from SOFTCO (Appendix 5f) explained why politicization of gate reviews influences demotivation moderately by stating, "Some of the project leaders are highly entrepreneurial and resourceful and find ways to go around project reviews to meet their requirements. If gate reviewers object to the purchase of some new equipment, the project leader will lease the equipment from a supplier and do away with the need for seeking approval for the purchase." Such actions generally keep teams' morale high.

The respondent from INDAUTCO (Appendix 5i) added a new explanation for demotivation, "At times some managers do feel demotivated, but it is not a result of politics. These are mostly technical managers who don't like the criticism of their technical work during project reviews." 
Likewise, the respondent from ELECTCO (Appendix 5j) explained that although the low level of politicization doesn't lead to demotivated teams, demotivation can come from other sources. He said, "If the project doesn't involve the latest technology as compared to other projects under development, it makes team members less enthusiastic. But, such demotivation does not last long. When the customer appreciates the work done on the project, it usually motivates the members once again."

In summary, there is strong consistency between interview responses and the quantitative findings that politicization increases demotivation. Yet, not all product development managers are equally demotivated. For example, engineers view projects as a learning experience and even if a project gets cancelled they can carry the knowledge gained on it to another project. Similarly, experienced managers may not be so demotivated by political meddling in projects because they accept it as a part of organizational life. However, demotivation doesn't just come from politics; it can come from other sources too.

\subsubsection{Moderating Effect of Conditionality on the Politicization of Gate Reviews -}

Demotivation Relationship. Consistent with the literature, it was hypothesized that conditionality would reduce the effect of politicization of gate reviews on demotivation of product development managers. However, the findings of the quantitative analysis do not support the hypothesis. 
Interview data from all twelve organizations is consistent with the results of the quantitative analysis and only half could be interpreted as supporting the hypothesis (Appendix 9).

In organizations with moderate and high politicization of gate reviews in which criteria conditionality is moderate or high (COMPCO - 5a, MACHCO - 5b, FILTCO - 5c, SEMCO $-5 \mathrm{~g}$ ), consistent with the quantitative results but not with the hypothesis, conditionality does not reduce the adverse effect of politicization on demotivation.

When organizations with moderate and high politicization of reviews have low criteria conditionality (ELECMACO - 5d, FABRICO - 5e, SOFTCO - 5f), it does not reduce the adverse effect of politicization of reviews, which is consistent with the results of quantitative analysis and with the hypothesized moderating effects. In other words, when there is moderate and high politicization, criteria conditionality does not moderate the effect of politicization on demotivation.

When asked if conditionality were high, would it make any difference, the respondent from ELECMACO (Appendix 5d) explained, "Conditional criteria don't affect technical managers because their source of motivation is learning about new technology. For nontechnical managers, conditional criteria do not make much difference because the negative effect of political gate reviews can be quite overwhelming for them." 
Similarly, in organizations with low politicization of reviews, and moderate conditionality, (ELECTCO - 5j, APPCO - 5k), consistent with the quantitative findings but not with the hypothesis, conditionality does not reduce demotivation. When such organizations (with low politicization of reviews) have low conditionality (COMAPPCO - 5h, INDAUTCO - 5i, HARSOCO - 51), it does not reduce the adverse effect of politicization, which is consistent with the results of quantitative findings and can be interpreted as supporting the hypothesized moderating effect.

When asked to explain why conditionality does not reduce the demotivation of product development managers despite moderate or highly politicized reviews, a similar response from many respondents was that politicization of gate reviews so demotivates product development managers that conditional criteria do not really provide any relief.

The respondent from FILTCO (Appendix 5c) provided an opposite view that sometimes high conditionality can worsen demotivation. For example, he said, "In my organization, even if the criteria are not met, there are no serious consequences and projects can still go through. This type of situation doesn't help reduce demotivation; instead sometimes it can have the opposite effect."

On the whole, there is strong consistency between interview responses and the quantitative results that conditionality does not moderate the effect of politicization on demotivation. Several respondents said that conditional criteria offer virtually no relief from the strongly demotivating effect of politicization of gate reviews. Sometimes, 
conditionality can even worsen demotivation if there are no serious consequences for not meeting the criteria at a later stage.

\subsubsection{Findings for Sub-Model 4}

7.2.4.1 Effect of Demotivation on Innovativeness of New Products. Interview data from all twelve organizations shows that demotivation adversely affects innovativeness of new products, which is consistent with the quantitative results and the hypothesis (Appendix 10).

There was general agreement among respondents that motivation is important for the development of innovative products. However, the respondent from SEMCO (Appendix $5 \mathrm{~g})$ explained why the moderate level of demotivation in his organization only somewhat reduces innovativeness by adding that product development managers who take politics in their stride and thus are less demotivated, do not have a negative influence on innovativeness.

\subsubsection{Moderating Effect of Technological Turbulence on the Demotivation -}

Innovativeness Relationship. It was hypothesized that technological turbulence would worsen the adverse effect of demotivation on innovativeness. This is supported by the results of quantitative analysis. However, the interview data presents a nuanced and insightful picture. Interview data from a little less than half the organizations ( 5 out of 
12 ) is consistent with the results of quantitative analysis and with the hypothesis (Appendix 10).

Out of seven organizations with moderate to high levels of demotivation, in only two cases (COMPCO - 5a, SEMCO - 5g) with high technological turbulence, the effect of demotivation on innovativeness is worsened by technological turbulence, which is consistent with the quantitative findings and the hypothesis. The respondent from SEMCO (Appendix 5g) elaborated on his response by saying, "If there is technological volatility, demotivated product development teams are likely to be overwhelmed by the resulting uncertainty and confusion and thus will find it difficult to develop innovative new products." Similarly, the respondent from COMPCO (Appendix 5a) said, "Demotivated teams find it difficult to incorporate the latest technologies in the product and when they do so, they are sometimes late to the market, so it harms innovativeness."

In the other two cases with high levels of technological turbulence (ELECMACO - 5d, SOFTCO - 5f), technological turbulence reduces the adverse effect of demotivation on innovativeness, which is inconsistent with both quantitative results and the hypothesis. The respondents from these organizations were told that quantitative findings contradict their responses. They were asked to explain why they had experienced a positive effect. The respondent from ELECMACO (Appendix 5d) provided a nuanced explanation. Demotivation varies depending on the background of product development managers. As discussed earlier, this respondent explained that engineers and technical experts are less demotivated by technological change than non-technical development team members. 
The technical experts are likely to see technological turbulence as an opportunity and since in this organization product development teams have more technical people, this positive outlook on technological turbulence energizes the team and thus spurs more innovativeness. The other respondent from SOFTCO (Appendix 5f) remarked, "Entrenched or well established organizations can view technological volatility as a problem, but newer set ups in fast moving industries like ours will generally see it as an opportunity to be more creative." This positive view of technological turbulence leads to more innovative outcomes.

Further, the respondent from ELECMACO (Appendix 5d) explained why the results of the quantitative analysis show that technical turbulence worsens the effect of demotivation on innovativeness, "Non-technical managers usually see technology turbulence as more of a problem and threat than technical managers - which can worsen the effect of demotivation on innovativeness. If the project teams in an organization have more non-technical members, the overall effect of technological volatility can be negative."

In the remaining three cases with moderate to high levels of demotivation (MACHCO $5 b$, FILTCO $-5 c$, FABRICO - 5e), technological turbulence was low and did not influence the demotivation-innovativeness relationship. This is consistent with the quantitative findings and the moderating hypothesis. 
When asked what would happen if technological turbulence were high, the respondent from FABRICO (Appendix 5e) said that even though engineers and technical people on teams might look at technological change with excitement which can make them develop innovative new products, the real technical people are few in teams. However, the nontechnical people who are already demotivated, may get further demotivated when they encounter technological turbulence. So the overall effect of technological turbulence will be negative.

The remaining five organizations out of 12 have low demotivation among product development managers and they have moderate to high levels of technological turbulence (COMAPPCO - 5h, INDAUTCO - 5i, ELECTCO - 5j, APPCO - 5k, HARSOCO - 5l). In all these cases, technological turbulence plays a positive role and helps reduce the adverse effect of demotivation on innovativeness, which is inconsistent with both quantitative results and the moderating hypothesis.

When asked to explain this contradiction, the respondents offered several different explanations. For example, the respondent from ELECTCO (Appendix 5j) said, "If changes in technology are rapid, they do frustrate product development team members and thus adversely affect their ability to innovate, but this happens less often. Since in our organization, teams are less demotivated, they are less frustrated by technological volatility." Thus, technological turbulence doesn't have an adverse effect. 
In HARSOCO (Appendix 5l) managers have less demotivation, and they view technological turbulence as an opportunity and thus come up with more innovative products. APPCO (Appendix 5k) is at the cutting edge of technology and introduces the latest products in the market. Their own induced technological turbulence favours them in developing innovative products.

Similarly, in COMAPPCO (Appendix 5h) the level of demotivation is low and product development teams are keen to learn about changes in technology and view the learning process as fun. This increases the team's enthusiasm and gives an impetus to innovation.

The respondent from INDAUTCO (Appendix 5i) offered an interesting explanation, "Technological volatility is a double-edged sword. If technology changes earlier in the development process, it can be seen as an opportunity to develop cutting edge products. But, if technology changes when the project is almost complete, many parts of the product need to be redesigned. These changes can frustrate product developers and can have an adverse effect on innovativeness. But more often than not, technological change is beneficial."

On the whole, in some cases technological turbulence worsens the effect of demotivation on innovativeness, and in others it has an opposite effect. According to several respondents, when the effect is worsened, it happens primarily because rapid technological changes overwhelm demotivated product development managers. On the other hand, regarding the positive effect of technological turbulence, several respondents 
explained that technology turbulence is often viewed positively as an opportunity by their product development managers. Such a positive view energizes and excites the managers, which leads to more innovative outcomes. Elaborating on this positive view of rapid technological changes, respondents offered explanations such as, their technical experts enjoy technology changes, in fast moving industries like theirs, technology change is generally seen as an opportunity, and their firm is the one which introduces technological changes and thus benefits from it.

\subsubsection{Moderating Effect of Market Turbulence on the Demotivation-Innovativeness}

Relationship. It was hypothesized that market turbulence would worsen the adverse effect of demotivation on innovativeness. However, results of quantitative analysis show that market turbulence reduces the adverse effect of demotivation on innovativeness. The explanations offered by the respondents are similar to the ones provided for the moderating effect of technological turbulence. Interview data from three-fourths of the organizations ( 9 out of 12) is consistent with the results of quantitative analysis and a little more than half of the responses ( 7 out of 12) can be interpreted as supporting the moderating hypothesis (Appendix 10).

Out of seven organizations with moderate to high levels of demotivation there are four cases with high levels of market turbulence. Among these, in only one organization (SOFTCO - 5f) market turbulence reduces the adverse effect of demotivation on innovativeness, which is consistent with quantitative results but contradicts the moderating hypothesis. In the remaining three cases (COMPCO $-5 a, M A C H C O-5 b$, 
and SEMCO $-5 \mathrm{~g}$ ), market turbulence worsens the effect of demotivation on innovativeness, which contradicts the empirical findings but is consistent with the hypothesis.

Further, among the organizations with moderate to high demotivation, there are three cases of low market turbulence. In all these cases (FILTCO - 5c, ELECMACO - 5d, and FABRICO - 5e), consistent with both the quantitative results and the hypothesis, low market turbulence does not affect the demotivation-innovativeness relationship.

When asked what would happen if market turbulence were high, the respondent from FABRICO (Appendix 5e) explained, "When market turbulence is high, new competitors are coming into the industry and customers demand newer things in the product, it can be seen as a challenge by all the members of the team. Market turbulence can create an external threat or challenge which has to be overcome. Such a challenge can unify, reenergize, and spur the team to do good work even though they may have been demotivated earlier." Thus, market turbulence can lead to more innovative new products when product development teams are demotivated, which contradicts the hypothesized relationship.

In the remaining five organizations with low demotivation, there are four cases with moderate to high market turbulence (INDAUTCO - 5i, ELECTCO - 5j, APPCO - 5k, and HARSOCO - 51). In these cases market turbulence reduces the adverse effect of demotivation on innovativeness, which is consistent with quantitative findings but 
contradicts the hypothesis. Furthermore, in organizations with low demotivation, there is one case with low market turbulence (COMAPPCO - 5h). In this case, a low level of market turbulence doesn't affect the demotivation-innovativeness relationship, which is consistent with the quantitative results and the moderating hypothesis.

When asked to explain, the respondents offered a variety of explanations for consistency or inconsistency with the quantitative results and hypothesized effects. Regarding consistency with the quantitative findings but inconsistency with the moderating hypothesis of market turbulence, the respondent from SOFTCO (Appendix 5f) said, "If markets and customer requirements change quickly, they create opportunities to come up with creative new products. These opportunities also create excitement in the team to effectively exploit them." The respondent from ELECTCO (Appendix 5j) had a similar response.

Regarding inconsistency with the quantitative findings and consistency with the moderating hypothesis of market turbulence, the respondent from COMPCO (Appendix 5a) said that market volatility basically occurs because customers change their requirements. He explained, "The products in our organization are usually made to specific customer requirements. Customers often say that they prefer products with new technologies. However, when it comes to actual implementation, customers sometimes can be quite risk averse and thus do not easily accept new technologies." This sudden change in customer requirements sometimes leads teams to reuse older technologies. While product development teams can look for an alternative acceptable new technology, 
their demotivation makes it difficult to identify and use such technologies - thus adversely affecting the innovativeness of new products.

Similarly, the respondent from MACHCO (Appendix 5b) said, "When customer requirements change rapidly, demotivated teams often do not change their products in response. Thus, such products are not latest enough in satisfying customers' changed requirements," (i.e., they are not as innovative).

Likewise, the respondent from SEMCO (Appendix 5g) when told that his response about the moderating effect of market turbulence contradicts the quantitative findings, said, "It is possible that those product development managers who are less demotivated, because they take political influence in their stride, might be able to use market volatility as an opportunity and thus have a positive effect on innovativeness." As he had said earlier, for some experienced product development managers, demotivation may not last long because they accept political meddling as a part of organizational reality.

On the whole, interviews suggest that in some cases, market turbulence reduces the adverse effect of demotivation on innovativeness (has a positive influence), and in few others it has an opposite effect. When the effect is positive, it happens primarily because market turbulence is viewed as an opportunity by product development managers. Also, in a turbulent market, competitors come and go, and customers demand newer things, which can be viewed as a threat and a challenge to be overcome. Such a view energizes and spurs the managers to develop more innovative products even though they may have 
been demotivated earlier. On the other hand, some team members, who take political meddling in their stride, may not be as demotivated and thus may be able to use rapid market changes to develop innovative products.

In contrast, the negative effect of market turbulence seems to occur basically because rapid market changes overwhelm demotivated product development managers and thus worsen the innovativeness of the new products they develop. In effect, the underlying reasons for the moderating effect of market turbulence are more nuanced than the ones that have been offered in the hypothesis based on the literature. Perhaps, factors such as the composition of the team (how experienced and mature its members are) and the leadership of the team (which can make the team view rapid market changes as a challenge) influence how market turbulence is viewed and how it affects the product development managers' response. It seems that taking such factors into account will better capture how things happen in organizations.

\subsubsection{Findings for Sub-Model 5}

7.2.5.1 Effect of Innovativeness on Performance. Interview data from all twelve organizations shows that innovativeness leads to improved market performance of new products, which is highly consistent with both the quantitative results and the hypothesis (Appendix 11). 
The respondent from FILTCO (Appendix 5c) explained the moderate effect of low innovativeness on performance by stating, "In our industry, true innovativeness is a rarity and customers do not seem to care about the bells and whistles you can put on a product." The respondent from SOFTCO (Appendix - 5f), while supporting the hypothesis, said that there can be a lag between the introduction of an innovative product and its performance, because such products can take time to show solid profitability. The respondent from SEMCO (Appendix - 5g) qualified his support for the hypothesis by saying that the effect of innovativeness may be limited sometimes, because there are other factors affecting performance.

In sum, while in general innovativeness has a positive effect on performance, the interview responses suggest that in some situations this relationship may not be that strong. For example, this relationship may be weakened if customers are not responsive to innovative products, or when other factors play a more important role (such as delivery time, price, etc.).

\subsubsection{New Insights}

Several new insights were obtained from the interviews. They relate to a variety of issues including the factors that keep organizational power and politics low, different perception of technical versus non-technical product development team members about environmental turbulence, and problems with the Stage-Gate process. These insights are discussed below. 


\subsubsection{Factors that Keep Organizational Politics and Power Accumulation Low.}

A source of problems for new products developed using the Stage-Gate process is the political climate in organizations and its adverse influence on the gate reviews. However, it doesn't seem easy to reduce this adverse effect. Rigorous gate review criteria and an entrepreneurial/innovative culture are ineffective in minimizing the influence of politics. These observations are supported by the quantitative findings of this dissertation. Given the difficulty of containing this adverse effect, the answer seems to lie in bringing down the level of politics in the organization in the first place - if that is feasible.

In several organizations, managerial actions keep the level of politics low. This in turn reduces power acquisition and politicization of reviews. Three interesting insights emerged from the interviews about how managers can keep the level of politics low in organizations.

One such factor is having a close knit organization that is highly connected across departments and vertically across management levels, and is focused on excelling in the market. Such an organization seems to keep the level of politics in the organization low and as a result, power acquisition and politicization are also minimized (APPCO - 5k).

The second factor appears to be the direct involvement of the owners or the top organizational leaders in the product development process and reviews. Such involvement is more likely to occur in a medium-sized, privately held company. It can 
keep the tendency to acquire power and politicize gate reviews under check (INDAUTCO - 5i).

The third factor which can keep the level of politics low is a serious threat to the organization. For example, in one of the organizations there has been a severe threat to the organization's survival, its sales have fallen and there has been fear that the organization might close down. Thousands of employees have been laid off and so the remaining employees know that they have to do their best to pull the organization out of its difficulties. As a result, there is very little politics in the organization or in its StageGate process. Thus, a threat to the organization can be a potent force that can make the organizational climate less political and through it reduce senior managers' tendency to accumulate power and politicize gate reviews. Organizational leaders can't invent such a threat but if one exists naturally, they can play it up to keep politics out (HARSOCO -51).

The above three and other possible drivers of low organizational politics need to be studied in future research on politics and innovation.

\subsubsection{Powerful Senior Managers and Politicization of Gate Reviews. The literature} suggests that if review criteria are strict and objective, extraneous influences are less likely to enter the Stage-Gate process. However, the interviews indicate that if powerful senior managers want to get certain projects approved, they will somehow get them approved, regardless of the rigour of the review criteria (COMPCO - 5a, MACHCO 5b, FILTCO - 5c, ELECMACO - 5d, FABRICO - 5e, SOFTCO - 5f, and SEMCO - 
$5 \mathrm{~g}$ ). They can use several means to achieve their political objectives. For example, it emerged that for the projects they favour, senior managers can have project managers make promises at gates that the criteria will be successfully met later. Subsequently, when those projects don't fulfill promises, these senior managers will intervene and protect project managers from any consequences. For the projects senior managers don't favour, they can cast doubt on their likelihood of succeeding by saying the ROI figures for the project seem unrealistic and unachievable (SEMCO - 5g).

\subsubsection{Factors that Keep Politicization of Gate Reviews Low. As just discussed, factors} that were expected to mitigate powerful managers' influence on the politicization of gate reviews do not appear to work effectively. However, the interviews identify three other factors that may work in influencing the politicization of reviews.

First, if new products are developed based on the customers' requirements (e.g., the customer dictates the product's features, specifications, and technologies to be used), then political dynamics might be different at gate reviews. It is likely to be more difficult to delay, demand major changes in, or kill a project for political reasons if specifications and design are driven by customer requirements. On the other hand, the implicit assumption in the conceptual model is that new products are developed primarily based on the product development teams' view of what customer needs are. Future studies on organizational politics in new product innovation can focus on customized products to generate new insights about the effect of power acquisition and the politicization of reviews (COMPCO - 5a and ELECTCO - 5j). Similarly, it will be interesting to 
understand in future research how power accumulation influences the politicization of gate reviews in organizations which are truly customer oriented.

The second factor that appears to influence the politicization of reviews is whether products are mere upgrades of existing products or new products. Senior managers may be less interested in influencing product upgrades because they don't bring them any major benefit; hence reviews may be less politicized for such products. However, this is a project level issue and future project level studies on politics and innovation may want to take this factor into account (COMAPPCO - 5h).

The third factor is an industry level innovation focus. To remain competitive in an industry where there is a strong innovation emphasis, an organization has to focus all its energies on innovation. Such a focus prevents senior managers from furthering their political interests by influencing project reviews in their favour (SOFTCO $-5 f$ ).

\subsubsection{Differences among Senior Managers and Their Motivations for Power}

Accumulation. One of the interesting insights that emerged is that there is a range of senior managers - some are top level managers and others are more middle-level managers (yet considered senior). Between them, the middle-level managers may have a greater tendency to accumulate power. While some of the senior managers may be driven by the desire to acquire more control and influence, these middle managers may be motivated more by their need for security and advancement in the organization (ELECMACO - 5d). 
This insight has implications for how slack influences the political climate-power accumulation relationship. For example, when the organization is profitable and there are surplus resources available, the middle-level managers feel less insecure about their jobs and thus try less to acquire power. Thus, high slack may not enhance the effect of a political climate on power accumulation where middle managers are involved in product review processes (ELECMACO - 5d).

\subsubsection{Different Perceptions of Technical vs. Non-Technical Product Development}

Managers Regarding Environmental Turbulence. In the product development literature, it is generally assumed that the entire product development team will have more or less the same view of environmental turbulence. However, some of these interviews have revealed that technical and non-technical members of the team can have different perceptions of environmental turbulence. For example, it has emerged that technical members of the team can view technical changes as an opportunity that can help them develop cutting edge products incorporating the latest technology (ELECMACO $-5 \mathrm{~d}$ ).

On the other hand, non-technical members can be overwhelmed by rapid technical changes, particularly when these individuals are demotivated to begin with. Since nontechnical members may outnumber technical experts on teams, overall teams may be negatively impacted by technological turbulence (FABRICO - 5e). This insight is consistent with the quantitative finding and the hypothesis that technological turbulence worsens the impact of demotivation on innovativeness. 
In contrast, market turbulence can be viewed as a threat by the entire team (i.e. both technical and non-technical members) and can thus energize the entire team to develop a superior product. In effect, market turbulence can lead even a demotivated team to produce more innovative outcomes. This insight offers an explanation for the quantitative finding that market turbulence positively moderates the relationship between demotivation and innovativeness (FABRICO - 5e).

\subsubsection{Role of the Project Leader in Influencing Demotivation. It also emerged that} project leaders can play an important role in minimizing the adverse effect of power and politics. For example, an entrepreneurial and resourceful leader can obtain the equipment required for a project through other means and thus go around some of the obstacles created at gate reviews for obtaining that equipment - which helps keep the project team's morale high (SOFTCO $-5 \mathrm{f}$ ). This dissertation is an organizational level study, but future project level research can study the role of the project leader in moderating the effect of power and politics.

7.2.6.7 Role of Individual Level Factors. From the interviews it also emerged that politicization of gate reviews may not demotivate all product development managers equally. For some experienced project managers this demotivation may not last long because they tend to accept political meddling as a part of organizational reality. This insight has implications for how product development managers will respond to environmental turbulence. For example, product development managers who are less demotivated, because they accept political influence as a part of organizational reality, 
may see environmental turbulence in a positive light and may use it to come up with more innovative products $(\mathrm{SEMCO}-5 \mathrm{~g})$.

While this factor cannot be taken into account in this dissertation because of its focus at the organizational level, future studies on politics and innovation at the individual and project levels may want to take it into consideration.

\subsubsection{Other Factors Influencing the Impact of Technological Turbulence. The stage of} the product development process at which technological changes emerge can influence the impact of these changes on the outcome of product development. If new technologies emerge earlier in the development process, they can provide opportunities to product designers to develop cutting edge products with the latest technology. However, if technology changes appear much later in the development process when the project is almost complete, many parts of the product need to be redesigned if the latest developments need to be incorporated into it. Such late changes often frustrate product development managers and can have an adverse effect on innovativeness. In effect, technological changes can act as a double edged sword - they can either help or harm innovativeness of the product depending on when they emerge (INDAUTCO - 5i). This issue needs more attention in future project level studies.

Organizations differ in terms of whether they use the latest technology or not, and this can influence how technological changes affect new product development. For example, it emerged that an organization may be the source of technological volatility in the 
industry because it usually introduces the latest, cutting edge technologies in its products. Its own induced technological turbulence can favour the organization in developing more innovative products than others in the industry. Such an organization is not adversely affected by technological turbulence (APPCO - 5k). Once again, this issue needs to be examined in future studies on innovation and environmental turbulence.

7.2.6.9 Problems with the Stage-Gate Process. The interviews identified two important problems with the Stage-Gate process. First, the Stage-Gate process can hamper the development of innovative new products. The process is intended to minimize risk and thus, mainly those projects that are less risky make it through the gates. Highly innovative products, by their very nature, involve risk and so usually don't make it through (MACHCO - 5b). Future project level studies on Stage-Gate can examine this issue in depth.

Second, the Stage-Gate process, like Six-Sigma and House of Quality, assumes that you can capture customers' requirements at the beginning of the product development process and freeze them. However, customer requirements sometimes evolve over time. It is important that product development teams learn about these evolving requirements and incorporate them in the new product otherwise the product will not satisfy the most current requirements of the customer. The Stage-Gate process in its current form does not leave room for such learning to take place. Thus the process needs to be modified to permit learning to occur. The focus of the reviews needs to change from checking boxes to knowledge creation (COMPCO - 5a). Although some preliminary work on learning in 
the Stage-Gate process has been done (Hutchins and Muller 2012), more research on how gates can be made learning oriented needs to be conducted. 


\section{DISCUSSION, IMPLICATIONS, AND CONCLUSIONS}

\subsection{Discussion}

The purpose of this dissertation is to examine the existence and effects of organizational politics and power accumulation on the Stage-Gate process and outcomes such as the motivation of product development managers and the innovativeness and performance of new products. It also identifies the factors that mitigate or enhance these effects of politics and power and assesses their influence.

One of the objectives of this dissertation is to examine the extent to which a political organizational climate leads to the accumulation of power by senior managers affected by new product development. Research indicates that a political climate in the organization can lead interest groups to strive for power over others (Galang and Ferris 1997). Does this mean that organizational politics increases power acquisition by senior managers in various departments who have the potential to influence the Stage-Gate process? Results from both the quantitative and qualitative studies show that a political organizational climate does lead to power accumulation by senior managers.

Another objective of this dissertation is to determine the degree to which organizational structural factors (slack and centralization) moderate the effect of a political organizational climate on power accumulation by senior managers. This issue deserves attention because although research indicates that organizational factors, such as 
centralization and slack, play a role in organizational power and political dynamics (Eisenhardt and Bourgeois 1988; Tjosvold and Poon 1998), it is not known if these factors influence the extent to which senior managers become more powerful in a political climate. The quantitative results suggest that both centralization and slack lead to increased power accumulation by senior managers in a political organizational climate. The qualitative findings also provide good support for the quantitative results.

Further, this dissertation examines the extent to which power accumulation by senior managers leads to the politicization of gate reviews. Although the literature suggests that gate reviews offer an opportunity to influence new products developed using the StageGate process (Cooper, Edgett, and Kleinschmidt 2002b), it is not well understood if powerful senior managers influence and interfere in gate reviews thereby politicizing them. Results from both the quantitative and qualitative studies show that powerful senior managers influence gate reviews and politicize them. Furthermore, the test of mediation indicates that in addition to an effect through power accumulation, a political organizational climate also directly makes gate reviews political.

Next, this research assesses the degree to which rigorous gate review criteria (i.e., strict and objective criteria) moderate the effect of power accumulation on politicization of gate reviews. There seems to be a belief in the Stage-Gate literature that if gate reviews are conducted using strict and objective criteria, projects will be evaluated solely on their merit rather than on extraneous considerations such as power and politics. This dissertation challenges this belief and examines if the influence of powerful managers on 
the politicization of gate reviews is affected by strictness and objectivity of the review criteria. Contrary to expectation, the findings of both the quantitative and the qualitative studies indicate that strict and objective review criteria are unable to prevent powerful managers from politicizing gate reviews. The qualitative interviews provide an explanation for the ineffectiveness of strict and objective criteria by highlighting that if powerful managers are really against a project, they somehow find a way to create problems for it. For example, they can cast doubt on the ROI figure of a project and call it unrealistic and unachievable. Similarly, if they are in favour of a project, they get it through even if that involves asking the project manager to make promises at the reviews which may not be ultimately fulfilled.

Another objective of this research is to determine the extent to which the entrepreneurial/innovative culture of the organization moderates the effect of power accumulation on politicization of gate reviews. If an organization is strongly innovation oriented, it may influence the extent to which senior managers play politics to influence or control the development of new products. However, similar to the role of review criteria, it is not known if the effect of powerful managers on the politicization of gate reviews is moderated by the entrepreneurial/innovative orientation of the organization. Again, contrary to expectation, the findings of both quantitative and qualitative studies suggest that an entrepreneurial/innovative culture plays no role in altering the influence of powerful managers on the politicization of gate reviews. The qualitative interviews indicate that powerful managers have their own agendas that they want to pursue and an entrepreneurial/innovative culture is ineffective in influencing that pursuit. In other 
words, the interests of powerful managers are so strong that they are not easily deterred by an entrepreneurial/innovative culture.

This research also examines the degree to which politicization has a demotivating effect on the product development managers who negotiate their products through the StageGate process. Research on politics and its effect on employees highlights that politics can have an adverse influence on employee morale (Drory 1993; Kacmar et al. 1999). However, in the context of the Stage-Gate process, it needs to be studied if politicization of gate reviews adversely affects the morale or motivation of product development managers. Both quantitative and qualitative findings show that politicization of gate reviews does demotivate product development managers. Also, the test of mediation highlights that in addition to an effect through politicization of gate reviews, power accumulation also directly influences demotivation.

The dissertation also assesses the extent to which the conditional nature of the review criteria moderates the relationship between politicization of gate reviews and demotivating experience of product development managers. Conditional criteria have been added to the Stage-Gate process in order to introduce flexibility in gate reviews and thereby, among other things, minimize the frustration product development managers experience at these reviews (Cooper 1994). Can such criteria conditionality reduce demotivation among product development managers when gate reviews are politicized? The results of both quantitative and qualitative studies show that conditionality does not affect the relationship between politicization and demotivation. Many qualitative 
interviews highlighted that the politicization of gate reviews so strongly demotivates product development managers that conditional criteria do not provide any effective relief.

The next objective of this research is to determine the degree to which product development managers' demotivation has an adverse effect on the innovativeness of new products. Given that research suggests that motivation plays a key role in creativity (Amabile 1988), does the demotivation of product development managers hurt their ability to develop innovative new products? Again, both quantitative and qualitative findings show that demotivation adversely affects innovativeness of new products. Further, the results of mediation analysis suggest that the politicization of gate reviews affects innovativeness of new products only by demotivating product development managers, which highlights the pivotal role of demotivation in hurting the development of innovative products. It is important to understand how such demotivation can be minimized.

In addition, this research examines the extent to which environmental factors (i.e., market and technological turbulence) moderate the relationship between demotivated managers and the innovativeness of new products. Research indicates that the development of innovative new products is quite challenging. This challenge may be greater if the organization is operating in turbulent environments (e.g. in the presence of market and technological turbulence) and its managers are demotivated due to politicization of gate reviews. However, it is not known what role turbulent environments play in this regard. 
The results of the quantitative study reveal that technological turbulence worsens the adverse effect of demotivation on innovativeness. On the other hand, the qualitative interviews only provide moderate support for this negative effect of technological turbulence. Some interviews suggested that technological turbulence could in fact have a positive effect, i.e. it may reduce the adverse effect of demotivation on innovativeness. For example, it emerged that engineers and technical experts are less demotivated by technical change than non-technical members because technical experts see technological turbulence as an opportunity and get energized by it, thereby spurring innovativeness. However, if technical experts on teams are fewer than the non-technical members, the team on the whole may be overwhelmed by technological turbulence which may adversely affect the innovativeness of new products.

Also, contrary to expectation, the quantitative findings show that market turbulence plays a positive role, i.e. it reduces the adverse effect of demotivation on innovativeness. The qualitative interviews also provide good support for this positive effect of market turbulence. The interviews explain this positive effect by highlighting that when market turbulence is high, i.e., new competitors are coming into the industry and customers demand newer products, it can be seen as a challenge by all members of the team. In effect, market turbulence creates an external threat or challenge which can unify, reenergize, and spur the team to do good work even though it may have been demotivated earlier. 
Finally, another objective of this research is to assess the degree to which innovativeness affects the performance of new products. Both quantitative and qualitative results show that innovativeness does have a positive effect on performance. Further, the test of mediation indicates that demotivation among product development managers adversely affects the performance of new products only by reducing the innovativeness of such products. This once again confirms the critical role of innovativeness in the success of new products that the literature highlights (Amabile 1988; Crawford 1977).

However, the findings of this dissertation need to be interpreted keeping in view its limitations.

\subsection{Limitations}

This dissertation is a first attempt to integrate organizational politics, power and the Stage-Gate literature and to empirically investigate complex relationships between the concepts from these diverse streams of literature. As such, the results of the empirical investigation need to be interpreted with caution.

The research methodology for this dissertation includes both qualitative and quantitative components. Since data for the quantitative study already existed, the qualitative study was not able to inform the design of the survey questionnaire. Therefore, some of the new insights generated by the qualitative study could not be verified in the quantitative 
stage. Yet, these insights have been included in the dissertation as potentially important areas for future exploration.

Furthermore, the sample for this study mainly includes medium and large organizations. Most concepts related to the formal process of new product development, and Stage-Gate process, in particular, have been developed in the context of large and medium organizations (Cooper 2001; Mankin 2004; Millward and Lewis 2005). These concepts may not be as applicable to small companies. From a new product development perspective, small companies have very different set of circumstances than large and medium-sized companies and most of them do not usually follow a formal product development process (Millward and Lewis 2005). Thus, the lessons learned from this dissertation that draws on the concepts developed for medium and large companies and uses a data set that includes only medium and large companies cannot be extended with any surety to small companies.

The data for the various measures used in this dissertation was obtained from the same informant and this can create the problem of common method bias. The issue of common-method bias arising from the quantitative study design was addressed by Sethi and Iqbal (2008) in their use of the data for their research. A similar approach is being used in this dissertation. For example, as Sethi and Iqbal (2008) describe, to assess if this bias exists, the recommendation about examining the items that were used to measure the different concepts for semantic overlap was followed (Podsakoff and Organ 1986). In this dissertation, there is hardly any overlap in the items in different measures. In the 
factor analyses, various items loaded on the factors representing the expected concepts. Further, it is not possible to explain the existence of moderating effects by common method bias (Evans 1985). In addition, the results of the quantitative analysis have been mostly supported by qualitative interviews. Therefore, common method bias does not seem to be a cause for concern.

The sample size in the quantitative study is 120 , which is not large enough to permit testing of the entire framework as one model using structural equations modeling. Similarly, this sample size does not allow checking for industry-based sensitivity of findings. Then, certain industries are not represented in the sample for both quantitative and qualitative studies. For example, pharmaceutical and bio-technology businesses were not a part of the sample. Thus, caution should be used while applying the findings of this study to businesses in such industries. Future research may want to replicate the findings in industries not included in this dissertation. The data for this research was collected from businesses in the US. Therefore, it may be useful to examine in future research how the findings extrapolate to other countries or cultures.

Finally, some other relationships of a direct or indirect nature and possibly feedback relationships may exist among the concepts in the model. However, this study has focused on the relationships that are believed to be important in capturing the influence of power and politics on the Stage-Gate process and are supported by the research literature. Future research may want to study such other relationships. 


\subsection{Research Implications}

This dissertation has several implications for research. To begin with, by integrating research in the areas of organizational power and politics, innovation, and the Stage-Gate system, this dissertation enriches the theoretical foundations of the Stage-Gate process. It has been argued in the literature that this process lacks a strong conceptual base. Future researchers can draw on the theoretical framework developed here to enrich their studies about the Stage-Gate process.

Next, it challenges the belief in the Stage-Gate literature that the process is insulated from external organizational influences including power and politics. Essentially, the StageGate literature seems to assume that if a well-designed system for the control and evaluation of new product development is introduced, it will work independently of the organizational context. More specifically, the Stage-Gate literature seems to assume that the review criteria and processes used at gates for evaluating new product development projects can insulate the development process from external organizational influences. For example, if the gate review criteria are strict and objective in nature, they will ensure that projects are evaluated on their merit and not on any other consideration including power and politics. However, the quantitative analysis in this dissertation finds that organizational politics makes senior managers acquire more influence or power, which they use to influence gate reviews in their favour and thereby politicize them. In effect, the Stage-Gate process is vulnerable to organizational power and politics. Furthermore, the quantitative results show that strict and objective review criteria are ineffective in 
preventing senior managers from influencing the review process for political considerations. Thus, strict and objective review criteria are unable to ensure that merit alone is used for evaluating projects. Hence, future studies on the Stage-Gate process need to actively take into account organizational influences while evaluating the effectiveness of the process. Particularly, the role of the gate review criteria needs to be more carefully examined because their effectiveness has now been called into question.

Furthermore, this dissertation shows that when power and politics creep into gate reviews, they end up hurting the outcome of the product development process. Specifically, the quantitative results suggest that the politicization of gate reviews demotivates product development managers, which in turn adversely affects the innovativeness of the firm's new products. Innovative new products lead to superior market performance. In essence, this dissertation for the first time has identified a specific step-by-step process through which organizational politics enters the Stage-Gate process and hurts the superiority and market performance of new products. Future research needs to identify more nuances related to this process and the other ways and means through which organizational politics influences the effectiveness of the StageGate process.

The literature generally assumes that organizational slack is good for innovation. This dissertation identifies a dark side of organizational slack. In a political organization, slack is used as a tool by senior managers to enhance their power, which in turn adversely affects the outcome of the Stage-Gate process. Although the role of slack in innovation 
has been studied before, it has been done mostly in a non-political context. The quantitative results of this dissertation suggest that there is a need to re-examine how slack operates in the presence of organizational politics.

Further, innovation research has shown that centralization is not good for the development of innovative new products (Zaltman, Duncan, and Holbek 1973). It is generally assumed that centralization takes decision making away from product development managers who are the real source of creativity. Yet, the Stage-Gate literature has often overlooked the role of centralization in influencing the effectiveness of the Stage-Gate process. The quantitative analysis in this dissertation finds that centralization hurts the Stage-Gate process and its outcomes but for a reason that is different from the one highlighted by the general innovation literature. In a political organization, centralization makes managers accumulate more power which, in turn, leads to greater politicization of gate reviews. Consistent with an earlier implication that the Stage-Gate literature cannot ignore organizational influences, future researchers need to take into account how centralization and other structural factors impact the effectiveness of the Stage-Gate process.

The qualitative interviews conducted as a part of this dissertation have generated several new insights. These insights have produced many avenues for future research that have already been discussed (see section 7.2.6). Among those insights, three need special attention and their research implications are being discussed below. 
The difficulty of containing the adverse effect of organizational power and politics on gate reviews has been highlighted by the results of the quantitative study. It emerged from qualitative interviews that the key to minimizing the politicization of gate reviews seems to lie in bringing down the level of politics in the organization. Three examples of how this can be done are: 1) having a highly connected organization that is strongly market focused, 2) direct involvement of top organizational leaders or owners in product development and reviews, and 3) by emphasizing a threat to the organization's survival, if it exists. Given the importance of keeping organizational politics low for successful innovation, future research studies may want to examine the effectiveness of the above three and other possible drivers of low politics.

In a similar vein, qualitative interviews suggested that besides keeping the level of organizational politics low, there are situations in which the politicization of gate reviews is likely to be directly minimized. For example, when products are developed based on customers' requirements, i.e. when the customer dictates the product features, specifications and technologies, it becomes difficult for senior manager to interfere in such projects and demand changes. Yet another factor that can minimize the level of politicization of gate reviews is a strong industry level emphasis on innovation which requires that an organization focus all its energies on innovation. Such a focus can prevent senior managers from furthering their political interests by influencing project reviews in their favour. Again, future studies may examine the effectiveness of these and other possible drivers of low politicization of gate reviews. 
The qualitative interviews also highlighted that the politicization of gate reviews may not demotivate all product development managers equally. For some experienced managers, this demotivation may be lower or not last long because they accept political meddling as a part of organizational reality. This is an important issue that needs attention because while it is difficult to prevent the politicization of gate reviews, if experienced/mature managers can take political meddling in their stride, it may have a positive effect on the outcome of the Stage-Gate process. Similarly, qualitative interviews indicated that technical experts are less demotivated by the politicization of gate reviews and by technological turbulence. In other words, product development teams will benefit if they include several technical experts, who seem to deal with the effects of power and politics and rapid technological change better. Finally, it emerged that resourceful project leaders can play a role in keeping product development managers motivated even in the presence of influences of power and politics on the Stage-Gate process. Future research may study the effectiveness of such factors.

\subsection{Managerial Implications}

This dissertation also has several implications for managers. To begin with, it warns top managers of a firm that they can't operate under the impression that the installation of a Stage-Gate system takes care of external organizational influences such as power and politics on the product development process. The problems that generally beset the traditional product development process are likely to continue even after setting up the 
Stage-Gate process. The problems created by power and politics still need to be taken care of and the Stage-Gate process should not be seen as a panacea.

Similarly, top managers have to realize that although the literature suggests that gate reviews need to be tough with strict and objective criteria so that projects are evaluated primarily on merit, such criteria are unable to achieve their purpose. In other words, the influence of organizational power and politics creeps into the process and politicizes gate reviews regardless of the strictness and objectivity of the criteria. Politicization of gate reviews harms the development of innovative new products which are usually coveted by firms because they lead to superior performance. Thus, managers should not be too eager to set up highly strict and objective criteria. It is important to realize that such criteria come at a cost, as they are known to make projects inflexible and hinder learning, and thus harm new product outcomes (Sethi and Iqbal 2008).

The results of this dissertation also warn top managers that a centralized structure can harm the new products developed using the Stage-Gate process, particularly if the organizational climate is political. Companies will be better off decentralizing their decision making when they install the Stage-Gate process. Thus, top managers have to resist the urge to control decision making in order to improve innovation because such control can have an opposite effect.

Guided by the innovation literature, top managers may believe that slack is necessary for innovation. However, the dissertation findings show that slack is likely to be used as a 
tool for power accumulation by senior managers in a political organizational climate which in turn adversely affects the new products developed through the Stage-Gate process. Yet, some level of slack is necessary for innovation, but excessive slack could become an instrument for power accumulation by senior managers.

A lesson from this dissertation is that it is not easy to prevent organizational power and politics from entering the Stage-Gate process. Therefore, the solution seems to be to keep the level of organizational politics low, if it is possible. Qualitative interviews suggest that in order to accomplish this, top managers may want to have a highly connected organization that is strongly market focused. In addition, their direct involvement in product development and reviews can minimize the level of politics because they can keep everyone focused on the task of innovation and prevent political interference by senior managers. Such involvement by top managers can also minimize the politics played by middle-level senior managers, who as it emerged may be more prone to politicking driven by a sense of insecurity. Finally, if there exists a threat to the organization, top management may be able to highlight it and use it to unify and energize the organization to counter the threat and in the process keep politics low.

Finally, if the organization is operating in a technologically turbulent environment, it is particularly important to have more motivated product development managers because less motivated managers in such an environment are unable to develop innovative new products. Since managers' lack of motivation is driven by organizational power and politics, it is particularly important to minimize such power and politics and prevent their 
influence from creeping into gate reviews. Moreover, if an organization is at the forefront of technology and is the one that introduces technological turbulence, it may benefit from such turbulence instead of being hurt by it. Similarly, if organizations are strongly focused on developing new products in response to customer requirements, they may be able to minimize interference in new product development projects for political reasons.

\subsection{Conclusions}

More and more firms are using the Stage-Gate system in hopes of improving their product development process and its outcomes. Although ignored by the Stage-Gate literature, power and politics are an integral part of organizational life and it is incorrect to assume that the Stage-Gate process can escape their influence. Therefore, any work that improves our understanding of how organizational power and politics influence the Stage-Gate process and how this influence gets enhanced or reduced makes an important contribution to research and practice of new product development. While this dissertation has made a first attempt toward addressing this issue, much more research is needed in this area.

Given the findings of this dissertation that the current design of the Stage-Gate process (e.g. the nature of the criteria and gate reviews) including some of the recent modifications (e.g., conditional criteria) is ineffective in preventing the influence of power and politics from creeping in, perhaps a relook at the design of the Stage-Gate 
process is needed. If such an attempt at redesign fails to even somewhat immunize the Stage-Gate process from power and politics, probably it needs to be examined if product development managers through their attitudes and actions can to some extent save projects from the adverse effect of power and politics. For example, the qualitative interviews suggest that experienced and mature product development managers tend to take political interference in their stride and continue to produce innovative outcomes. Similarly, the interviews highlight that technical experts view every project as a learning experience and thus are not so easily discouraged by political interference. In general, considering the importance of innovation in making companies competitive, research effort needs to continue toward finding ways of developing more innovative products through the Stage-Gate process and improving the effectiveness and the value of the Stage-Gate process to this end. 


\section{REFERENCES}

Aiken, Leona S. and Stephen G. West (1991), Multiple Regression: Testing and Interpreting Interactions. Newbury Park, CA: Sage Publications.

Allen, Robert W., Dan L. Madison, Lyman W. Porter, Patricia A. Renwick, and Bronston T. Mayes (1979), "Organizational Politics: Tactics and Characteristics of its Actors," California Management Review, 22 (Fall), 77-83.

Allison, Graham T. (1971). Essence of Decision: Explaining the Cuban Missile Crisis. Boston, MA: Little Brown Publishers.

Amabile, Teresa M. (1983), The Social Psychology of Creativity. New York: SpringerVerlag.

(1988), "A Model of Creativity and Innovation in Organizations," in Research in Organization Behavior, Vol 10, Barry M. Staw and L. L. Cummings, eds. Greenwich, CT: JAI Press Inc. 123-67.

Andrews, Jonlee and Daniel C. Smith (1996), "In Search of Marketing Imagination: Factors Affecting the Creativity of Marketing Programs for Mature Products," Journal of Marketing Research, 33 (May), 174-87.

Armstrong, J. Scott and Terry S. Overton (1977), "Estimating Nonresponse Bias in Mail Surveys," Journal of Marketing Research, 14 (August), 396-402.

Astley, W. Graham and Paramjit S. Sachdeva (1984), "Structural Sources of Intraorganizational Power: A Theoretical Synthesis," Academy of Management Review, 9 (January), 104-13.

Barczak, Gloria, Abbie Griffin, and Kenneth B. Kahn (2009), "Perspective: Trends and Drivers of Success in NPD Practices: Results of the 2003 PDMA Best Practices Study," Journal of Product Innovation Management, 26 (January), 3-23.

Bachrach, Peter and Morton S. Baratz (1962), "The Two Faces of Power," American Political Science Review, 56 (December), 947-52.

University Press. (1970), Power and Poverty. London: Oxford

Baron, Reuben M. and David A. Kenny (1986), “The Moderator-Mediator Variable Distinction in Social Psychological Research: Conceptual, Strategic, and Statistical Considerations," Journal of Personality and Social Psychology, 51 (December), 1173-82. 
Bartel,Caroline A. and Raghu Garud (2009), "The Role of Narratives in Sustaining Organizational Innovation," Organizational Sceince, 20 (January-February), 107-17.

Benfari, Robert. C., Harry E. Wilkinson, and Charles D. Orth (1986), "The Effective Use of Power," Business Horizons, 29 (May-June), 12-6.

Bourgeois III, L. J. (1981), "On the Measurement of Organizational Slack," Academy of Management Review, 6 (1), 29-39.

Brown, Shona L. and Kathleen M. Eisenhardt (1997), "The Art of Continuous Change: Linking Complexity Theory and Time-Paced Evolution in Relentlessly Shifting Organizations," Administrative Science Quarterly, 42 (March), 1-34.

(1998), Competing on the Edge: Strategy

as Structured Chaos. Boston, MA: Harvard Business School Press.

Buchanan, David A. (2008), "You Stab My Back, I'll Stab Yours: Management Experience and Perceptions of Organization Political Behaviour," British Journal of Management, 19 (March), 49-64.

and Richard Badham (2003), Power, Politics and Organizational

Change: Winning the Turf Game. London: Sage Publications.

Chesbrough, Henry (2003), Open Innovation: The Imperative for Creating and Profiting From Technology. Boston: Harvard Business School Press.

Cheung, Mike W.L. (2007), "Comparison of Methods of Handling Missing TimeInvariant Covariates in Latent Growth Models Under the Assumption of Missing Completely at Random," Organizational Research Methods, 10 (October), 609-34.

Cohen, Jacob (1988). Statistical Power Analysis for the Behavioral Sciences. Hillsdale, NJ: Lawrence Erlbaum Associates.

Cooper, Robert (1979), "The Dimensions of Industrial New Product Success and Failure," Journal of Marketing, 43 (3), 93-103.

(1980), "Project New Prod: Factors in New Product Success," European Journal of Marketing, 14 (5), 277-92.

(1986), Winning at New Products. Reading, MA: Addison -Wesley.

(1994), "Third-Generation New Product Processes," Journal of Product Innovation Management, 11(January), 3-14.

(1998), Product Leadership: Creating and Launching Superior New

Products. Reading, MA: Perseus Books. 
(2001), Winning at New Products: Accelerating the Process from Idea to Launch. Cambridge, MA: Perseus Publishing.

(2008), "Perspective: The Stage-Gate Idea-to-Launch Process - Update, What's New, and NextGen Systems," Journal of Product Innovation Management, 25 (May), 213-32.

and Elko J. Kleinschmidt (1987), "New Products: What Separates Winners From Losers," Journal of Product Innovation Management, 4(3), 169-84.

, Scott J. Edgett, Elko J. Kleinschmidt (2002a), "Optimizing the Stage-Gate Process: What Best-Practice Companies Do - 1" Research Technology Management, 45 (5), 21-27.

(2002b), "Optimizing the Stage-Gate

Process: What Best-Practice Companies Do - II" Research Technology Management, 45 (6), 43-49.

Crawford, Merle C. (1977), "Marketing Research and the New Product Failure Rate," Journal of Marketing, 41(April), 51-61.

(1987), "New Product Failure Rates: A Reprise," Research

Management, 30 (July-August), 20-24.

Cyert, Richard M. and James G. March (1963), A Behavioral Theory of the Firm, New Jersey: Prentice Hall.

Danneels, Erwin (2002), "The Dynamics of Product Innovation and Firm Competences," Strategic Management Journal, 23 (12), 1095-121.

and Rajesh Sethi (2011), "New Product Exploration under Environmental Turbulence," Organization Science, 22 (July-August), 1026-39.

Darr, Wendy and Gary Johns (2004), "Political Decision-Making Climates: Theoretical Processes and Multi-Level Antecedents," Human Relations, 57 (2), 169-200.

Deshpande, Rohit, John U. Farley, and Frederick E. Webster Jr. (1993), "Corporate Culture, Customer Orientation, and Innovativeness," Journal of Marketing, 57(January), 23-37.

Dess, Gregory G. and Donald W. Beard (1984), "Dimensions of Organizational Task Environments," Administrative Science Quarterly, 29 (March), 52-73.

Diamantopoulos, Adamantios and Heidi M. Winklhofer (2001), "Index Construction with Formative Indicators: An Alternative to Scale Development," Journal of Marketing Research, 38 (May), 269-77. 
Dougherty, Deborah and Cynthia Hardy (1996), "Sustained Product Innovation in Large, Mature Organizations: Overcoming Innovation-To-Organization Problems," Academy of Management Journal, 39 (5), 1120-153.

and Trudy Heller (1994), "Illegitimacy of Successful Product

Innovation in Established Firms," Organization Science, 5 (2), 200-18.

Drory, Amos (1993), "Perceived Political Climate and Job Attitudes," Organization Studies, 14 (1), 59-71.

Eisenhardt, Kathleen M. and L. J. Bourgeois III (1988), "Politics of Strategic Decision Making in High Velocity Environments: Toward a Midrange Theory," Academy of Management Journal, 31 (December), 737-90.

Erdogan Zafer B. and Michael J. Baker (2002), "Increasing Mail Survey Response Rates From an Industrial Population," Industrial Marketing Management, 31 (January), 65-73.

Ettlie, John E. and Jorg M. Elsenbach (2007), "Modified Stage-Gate Regimes in New Product Development," Journal of Product Innovation Management, 24 (January), 20-33.

Evans, Martin G. (1985), "A Monte-Carlo Study of the Effects of Correlated Method Variance in Moderated Multiple Regression Analysis," Organizational Behavior and Human Decision Processes, 36 (December), 305-23.

Fairholm, Gilbert W. (1993), Organizational Power Politics. Westport, Connecticut: Praeger Publishers.

Ferris, Gerald R., Gail S. Russ, and Patricia M. Fand (1989), "Politics in Organizations" in Impression Management in the Organization, Robert A. Giacalone and Paul Rosenfeld eds. Newbury Park, CA: Sage, 143-70.

, Dwight D. Frink, David C. Gilmore, and K. Michelle Kacmar (1994), "Understanding as an Antidote for the Dysfunctional Consequences of Organizational Politics as a Stressor," Journal of Applied Social Psychology, 24 (13), 1204-20.

Fornell, Claes and David F. Larcker (1981), "Evaluating Structural Equation Models with Unobservable Variables and Measurement Error," Journal of Marketing Research, 18 (February), 39-50.

French, John R. P. and Bertram Raven (1959), "The Bases of Social Power," in Studies in Social Power, Darwin Cartwright, ed. Ann Arbor, Michigan: Institute for Social Research, University of Michigan, 150-65.

Frost, Peter J. and Carolyn P. Egri (1990a), "Influence of Political Action on Innovation: Part 1," The Leadership and Organization Development Journal, 11 (1), 17-25. 
(1990b), "Influence of Political Action on Innovation:

Part II," The Leadership and Organization Development Journal, 11 (2), 4-12.

(1991), "The Political Process of Innovation," in Research in Organizational Behavior, Vol. 13, Barry M. Staw and Larry L. Cummings, eds. Greenwich, CT: JAI Press Inc. 229-95.

and D. C. Hayes (1979), "An Exploration in Two Cultures of a Model in Political Behavior in Organizations" in Organizational Functioning in a CrossCultural Perspective, George William England, Bernhard Wilpert, Anant R. Negandhi eds. Kent State University: Comparative Administration Research Institute.

Galang, Maria Carmen and Gerald R. Ferris (1997), "Human Resource Department's Power and Influence Through Symbolic Action," Human Relations, 50 (November), 1403-26.

Gandz, Jeffery and Victor V. Murray (1980), "The Experience of Workplace Politics," Academy of Management Journal, 23 (2), 237-51.

Gao, Shengyi, Patricia L. Mokhtarian, and Robert A. Johnston (2008), "Nonnormality of Data in Structural Equation Models," Transportation Research Record: Journal of the Transportation Research Board, 2082, 116-24.

Gorchels, Linda (1996), The Product Manager's Handbook. Chicago, IL: NTC Publishing Group.

Gresov, Christopher and Carroll Stephens (1993), "The Context of Interunit Influence Attempts," Administrative Science Quarterly, 38 (June), 252-76.

Grewal, Rajdeep, Joseph A. Cote, Hans Baumgartner (2004), "Multicollinearity and Measurement Error in Structural Equation Models: Implications for Theory Testing," Marketing Science, 23 (June), 519-29.

Griffin, Abbie (1997), "PDMA Research on New Product Development Practices: Updating Trends and Benchmarking Best Practices," Journal of Product Innovation Management, 14, 429-58.

Gronlund, Johan, David R. Sjodin and Johan Frishammar (2010), "Open Innovation and the Stage-gate Process: A Revised Model for New Product Development," California Management Review, 52 (Spring), 106-31.

Hage, Jerald (1980), Theories of Organization, Form, and Transformation, New York: Wiley.

Hair, Joseph F., William C. Black, Barry J. Babin, Rolph E. Anderson and Ronald L. Tatham (2006), Multivariate Data Analysis. New Jersey: Pearson Prentice Hall. 
Hardy, Cynthia (1994), Managing Strategic Action, Thousand Oaks, California: Sage Publications.

(1996), "Understanding Power: Bringing About Strategic Change," British Journal of Management, 7 (March), 3-16.

and Stewart R. Clegg (1996), "Some Dare Call it Power," in Handbook of Organizational Studies, Stewart R. Clegg, Cynthia Hardy, and Walter R. Nord eds. London: Sage Publications, 622-41.

and Deborah Dougherty (1997), "Powering Product Innovation," European Management Journal, 15 (1), 16-27.

Harris, Lloyd C. (2001), "Market Orientation and Performance: Objective and Subjective Empirical Evidence from UK Companies," Journal of Management Studies, 38 (January), 17-43.

Harzing, Anne-Wit (2000), “Cross-National Industrial Mail Surveys,” Industrial Marketing Management, 29 (May), 243-54.

Herold, David M., Narayanan Jayaraman, and C R Narayanaswamy (2006), "What is the Relationship between Organizational Slack and Innovation?" Journal of Managerial Issues, 18 (Fall), 372-92.

Hogarth, Robin (1980), Judgement and Choice. Chichester, England: John Wiley and Sons.

Hurley, Robert F. and G. Tomas M. Hult (1998), "Innovation, Market Orientation, and Organizational Learning: An Integration and Empirical Examination," Journal of Marketing, 62 (July), 42-54.

Hutchins, Nate and Amy Muller (2012), "Beyond Stage-gate: Restoring Learning and Adaptability to Commercialization," Strategy and Leadership, 40 (3), 30-5.

Im, Subin and John P. Workman, Jr. (2004), "Market Orientation, Creativity, and New Product Performance in High technology Firms," Journal of Marketing, 68 (April), 11432.

Jaccard, James, Robert Turrisi, and Choi K. Wan (1990), Interaction Effects in Multiple Regression, Newbury Park, CA: Sage Publications.

Jaworski, Bernard and Ajay K. Kohli (1993), "Market Orientation: Antecedents and Consequences," Journal of Marketing, 57 (July), 53-70.

Jehn, Karen A. (1997), A Qualitative Analysis of Conflict Types and Dimensions in Organizational Groups," Administrative Science Quarterly, 42 (September), 530-57. 
Kahn, Kenneth (2001), Product Planning Essentials. Thousand Oaks, CA: Sage Publications, Inc.

Kanter, Rosabeth Moss (1983), Change Masters. New York: Simon and Schuster.

(1988), "When a Thousand Flowers Bloom: Structural, Collective, and Social Conditions for Innovation in Organizations," in Research in Organizational Behavior, Vol. 10, Barry M. Staw and L. L. Cummings, eds. Greenwich, CT: JAI Press Inc. 169-211.

Kacmar, K. Michelle and Robert A. Baron (1999), "Organizational Politics: The State of the Field, Links to Related Processes, and an Agenda for future Research," in Research in Personnel and Human Resources Management, Kendrith M. Rowland and Gerald R. Ferris eds. Greenwich: JAI Press Inc. 10, 1-39.

Kelley, George (1976), "Seducing the Elites: The Politics of Decision Making and Innovation in Organizational Networks," Academy of Management Review, 1 (July), 6674.

Kenny, David A., Kashy, Deborah A., and Bolger, N. Niall (1998), Data Analysis in Social Psychology. In: The Handbook of Social Psychology, Vol 2, Daniel T. Gilbert, Susan T. Fiske, \& Gardener Lindzey (eds.). Boston, MA: McGraw-Hill, 233-65.

Kimberly, John R. (1980), "Managerial Innovation," in Handbook of Organizational Design, Paul C. Nystrom and William H. Starbuck eds., Oxford University Press, N.Y. 84-104.

Kohli, Ajay K. and Bernard J. Jaworski (1990), "Market Orientation: The Construct, Research Propositions, and Managerial Implications," Journal of Marketing, 54 (April), $1-18$.

Leifer, Richard, Christopher M. McDermott, Gina Colarelli O'Connor, Lois Peters, Mark Rice, and Robert W. Veryzer (2000), Radical Innovation. Boston, MA: Harvard Business School Press.

Lukes, Steven (1974), Power: A Radical View. London: Macmillan.

Machiavelli, Niccolo (1977), The Prince. Translated and edited by Robert M. Adams, New York: Norton.

MacKinnon, David P., Amanda J. Fairchild, and Matthew S. Fritz (2007), "Mediation Analysis," Annual Review of Psychology, 58 (January), 593-614.

Mankin, Eric (2004), "Is Your Product-Development Process Helping or Hindering Innovation?" Strategy and Innovation, (November-December), 3-5. 
March, James G. and Herbert A. Simon (1958), Organizations. New York, John Wiley and Co. Inc.

Markus, Lynne M. (1983), "Power, Politics and MIS Implementation," Communications of the $A C M, 26(6), 430-44$.

McCracken, Grant (1988), The Long Interview. Newbury Park, CA: Sage Publications.

McDermott, Christopher M., and Gina Colarelli O'Connor (2002), "Managing Radical Innovation: An Overview of Emergent Strategy Issues," Journal of Product Innovation Management, 19 (November), 424-38.

Menon, Anil, Sundar G. Bharadwaj, Phani Tej Adidam, and Steven W. Edison (1999), Antecedents and Consequences of Marketing Strategy Making: A Model and a Test," Journal of Marketing, 63 (April), 18-40.

Miller, Brian K., Matthew A. Rutherford and Robert W. Kolodinsky (2008), "Perceptions of Organizational Politics: A Meta-Analysis of Outcomes," Journal of Business Psychology, 22 (3), 209-22.

Miller Danny and Peter H. Friesen (1983), "Strategy-Making and Environment: The Third Link," Strategic Management Journal, 4 (July-September), 221-35.

Millward, Huw and Alan Lewis (2005), "Barriers to Successful New Product Development within Small Manufacturing Companies," Journal of Small Business and Enterprise Development, 12 (April), 379-94.

Mintzberg, Henry (1983), Power In and Around Organizations. Englewood Cliffs: Prentice-Hall.

Studies, 22 (2), 133-54.

(1985), "The Organization as Political Arena," Journal of Management

Moch, Michael K. and Louis R. Pondy (1977), "The Structure of Chaos: Organized

Anarchy as a Response to Ambiguity," Administrative Science Quarterly, 22 (2), 351-62.

Muthen, Bengt and David Kaplan (1985), "A Comparison of Some Methodologies for the Factor Analysis of Non-Normal Likert Variables," British Journal of Mathematical and Statistical Psychology, 38 (November), 171-89.

Myers, Michael D. (2009), Qualitative Research in Business and Management. Thousand Oaks, CA: Sage Publications.

Myers, Raymond H. (1986), Classical and Modern Regression with Applications. Boston, MA: Duxbury Press. 
Narayanan, V. K. and Liam Fahey (1982), "The Micro-Politics of Strategy Formulation," The Academy of Management Review, 7 (January), 25-34.

Netermeyer, Richard G., William O. Bearden, Subhash Sharma (2003), Scaling

Procedures: Issues and Applications. Thousand Oaks, CA: Sage Publications..

Nunnally, Jum C. (1978), Psychometric Theory. New York: McGraw-Hill.

Nye, Lendell G. and L. A Witt (1993), "Dimensionality and Construct Validity of the Perceptions of Organizational Politics Scale (POPS)", Educational and Psychological Measurement, 53 (3), 821-29.

Opsina, Sonia (2004), “Qualitative Research," in Encyclopedia of Leadership, G. Goethals, G. Sorenson, J. MacGregor, eds. Thounsad Oaks, CA: Sage Publications.

O'Reilly, Charles (1989), "Corporations, Culture, and Commitment: Motivation and Social Control in Organizations," California Management Review, 31 (Summer), 9-25.

Osborn, Alex F. (1963), Applied Imagination. New York: Charles Scribner's Sons.

Perrow, Charles (1986), Complex Organizations. New York: Random House.

Peter, J. Paul (1981), "Construct Validity: A Review of Basic Issues and Marketing Practices," Journal of Marketing Research, 18 (2), 133-45.

Pettigrew, Andrew (1972), "Information Control as a Power Resource," Sociology, 6 (2), 187-204.

Tavistock Publications.

(1973), The Politics of Organizational Decision-Making, London:

and T. McNulty (1995), "Power and Influence in and Around the Boardroom," Human Relations, 48 (8), 845-73.

Pfeffer, Jeffrey (1981), Power in Organizations. Mansfield, MA: Pitman Publishing. (1992), Managing with Power. Harvard Business School Press, Boston: MA. and William L. Moore (1980), "Power in University Budgeting: A Replication and Extension," Administrative Science Quarterly, 25 (December), 637-53. and Gerald R. Salancik (1974), "Organizational Decision Making as a Political Process: The Case of a University Budget," Administrative Science Quarterly, 19 (June), 135-51. 
and

York: Harper and Row, Publishers.

(1978), External Control of Organization. New

Podsakoff, Philip M. and Dennis W. Organ (1986), "Self-Reports in Organizational Research: Problems and Prospects," Journal of Management, 12 (4), 53-44.

Preacher, Kristopher J. and Andrew F. Hayes (2004), "SPSS and SAS Procedures for Estimating Indirect Effects in Simple Mediation Models," Behavior Research Methods, 36 (November), 717-31.

Randall, Margorie L., Russell Cropanzano, Carol A. Bormann, and Andrej Birjulin (1999), "Organizational Politics and Organizational Support as Predictors of Work Attitudes, Job Performance, and Organizational Citizenship Behavior", Journal of Organizational Behavior, 20 (March), 159-74.

Rubin Leah H., Katie Witkiewitz, Justin St. Andre, and Steve Reilly (2007), "Methods for Handling Missing Data in the Behavioral Neurosciences: Don't Throw the Baby Rat out with the Bath Water," The Journal of Undergraduate Neuroscience Education, 5 (Spring), A71-A77.

Sarin, Shikhar and Christopher McDermott (2003), "The Effect of Team Leader Characteristics on Learning, Knowledge Application, and Performance of CrossFunctional New product Development Teams," Decision Sciences, 34 (4), 707-39.

Schmidt, Jeffrey B., Kumar R. Sarangee, and Mitzi M. Montoya-Weiss (2009), "Exploring New Product Development Project Review Practices," Journal of Product Innovation Management, 26 (September), 520-35.

Schoonhoven, Claudia Bird (1981), "Problems with Contingency Theory: Testing assumptions Hidden within the Language of Contingency "Theory"," Administrative Science Quarterly, 26 (September), 349-77.

Sethi, Rajesh (2000), "Superordinate Identity in Cross-Functional Product Development Teams: Its Antecedents and Effect on New Product Performance," Journal of the Academy of Marketing Science, 28 (3), 330-44.

, Daniel C. Smith, and C. Whan Park (2001), "The Effect of CrossFunctional Product Development Teams on the Innovativeness of New Consumer Products," Journal of Marketing Research, 38 (1), 73-85.

and Zafar Iqbal (2008), "Stage-Gate Controls, Learning Failure, and Adverse Effect on Novel New Products," Journal of Marketing, 72 (January), 118-34.

Sharma, Subhash, Richard M. Durand, and Oded Gur-Arie (1981), "Identification and Analysis of Moderator Variables," Journal of Marketing Research, 18 (August), 291-300. 
Simon, Herbert A. (1957), Models of Man: Social and Rational. New York: Wiley.

Smith, D. J. (2007), "The Politics of Innovation: Why Innovations Need a Godfather," Technovation, 27 (March), 95-104.

Stafford, James E. (1966), "Influence of Preliminary Contact on Mail Returns," Journal of Marketing Research, 3 (November), 410-11.

Tjosvold Dean and Margaret Poon (1998), "Dealing with Scarce Resources," Group \& Organization Management, 23 (September), 237-55.

Valle, Matthew. P. and Pamela L. Perrewe (2000), "Do Politics Perceptions Relate to Political Behaviors? Tests of an Implicit Assumption and Expanded Model," Human Relations, 53 (3), 359-86.

Van Kenhove, Patrick, Katrien Wijnen, and Kristof De Wulf (2002), "The Influence of Topic Involvement on Mail-Survey Response Behavior," Psychology and Marketing, 19 (March), 293-301.

Vigoda, Eran (2000), "Internal Politics in Public Administration Systems: An Empirical Examination of its Relationship with Job Congruence, Organizational Citizenship Behavior, and In-Role Performance," Public Personnel Management, 29 (2), 185-210.

Veryzer, Robert W. (1998), "Discontinuous Innovation and the New Product Development Process," Journal of Product Innovation Management, 15 (July), 304-21.

Welsh, Ann M., \& Allen E. Slusher (1986), "Organizational Design as a Context of Political Activity," Administrative Science Quarterly, 31 (September), 389-402

Witt, L. A., Michelle Kacmar, Dawn S. Carlson, and Suzanne Zivnuska (2002), "Interactive Effects of Personality and Organizational Politics on Contextual Performance," Journal of Organizational Behavior, 23 (December), 911-26.

Zaltman, Gerald, Robert Duncan, and Jonny Holbek (1973), Innovations and Organizations. New York: John Wiley.

Zhou, Kevin Zheng, Chi Kin Yim, and David K.Tse (2005), "The Effects of Strategic Orientations on Technology-and Market-Based Breakthrough Innovations," Journal of Marketing, 69 (April), 42-60. 
Appendices 


\section{Appendix 1 \\ Measures}

\begin{tabular}{|c|c|}
\hline $\begin{array}{l}\text { Concept } \\
\text { (Source) }\end{array}$ & Items \\
\hline $\begin{array}{l}\text { Political } \\
\text { Organizational } \\
\text { Climate } \\
\text { (New measure } \\
\text { based on Ferris, } \\
\text { Russ, and Fand } \\
\text { 1989; Fairholm } \\
\text { 1993; Witt, } \\
\text { Kacmar, } \\
\text { Carlson, and } \\
\text { Zivnuska 2002) }\end{array}$ & $\begin{array}{l}\text { In our organization, there are usually strong political undertones in most } \\
\text { major decisions. } \\
\text { Managers in our organization usually see things from a political perspective. } \\
\text { In my organization, there is little politics. (R) } \\
\text { Politics is ingrained deeply in our business unit. } \\
\text { Nothing can be done about the degree of politics that exists in our business } \\
\text { unit. } \\
\text { Politics often overshadows overall business unit interests. } \\
\text { Engaging in political behavior is the way to get things done in our business } \\
\text { unit. }\end{array}$ \\
\hline $\begin{array}{l}\text { Slack }^{\mathbf{a}} \\
\text { (Existing } \\
\text { measure from } \\
\text { Danneels and } \\
\text { Sethi 2011) }\end{array}$ & $\begin{array}{l}\text { In my business unit, most of our resources are locked up in current projects. } \\
\text { (R) } \\
\text { Our business unit has a reasonable amount of resources in reserve. } \\
\text { We have ample discretionary financial resources. } \\
\text { We can always find the manpower to work on new product development } \\
\text { projects. }\end{array}$ \\
\hline $\begin{array}{l}\text { Centralization }^{\mathbf{a}} \\
\text { (Existing } \\
\text { measure from } \\
\text { Jaworski and } \\
\text { Kohli 1993). }\end{array}$ & $\begin{array}{l}\text { There can be little action taken here until a senior manager approves a } \\
\text { decision. } \\
\text { A person who wants to make his/her own decisions would be quickly } \\
\text { discouraged here. } \\
\text { Even small matters have to be referred to someone higher up for a final } \\
\text { answer. } \\
\text { People here have to ask their boss before they do almost anything. } \\
\text { Any decision people make has to have their boss's approval. } \\
\text { People here feel that they are their own boss in certain matters. (R) } \\
\text { People here can make their own decisions without checking with anybody } \\
\text { else. (R) } \\
\text { How things are done around here is left up to the person doing the work. (R) }\end{array}$ \\
\hline
\end{tabular}




\begin{tabular}{|c|c|}
\hline $\begin{array}{l}\text { Power } \\
\text { Accumulation" } \\
\text { (New measure } \\
\text { based on Hardy } \\
\text { 1994; Hardy } \\
\text { and Clegg 1996) }\end{array}$ & $\begin{array}{l}\text { Enough resources are widely distributed to a variety of senior managers in } \\
\text { my business unit }(\mathrm{R}) \text {. } \\
\text { In my business unit, senior managers who control resources generally use } \\
\text { them to influence the actions of others to suit their own interests. } \\
\text { In my business unit, resources are widely distributed to various functional } \\
\text { areas.(R) }\end{array}$ \\
\hline $\begin{array}{l}\text { Power } \\
\text { Accumulation } \\
\text { (continued) }\end{array}$ & $\begin{array}{l}\text { Certain senior managers in my business unit have significant control on } \\
\text { strategic and operational decisions. } \\
\text { The strategic priorities and agenda are set in an open and participative } \\
\text { manner. (R) } \\
\text { Decision criteria for important issues are controlled by only a few senior } \\
\text { managers. } \\
\text { Certain departments have the power to veto virtually any proposal that } \\
\text { they don't agree with. }\end{array}$ \\
\hline $\begin{array}{l}\text { Strict Criteria } \\
\text { (Existing } \\
\text { measure from } \\
\text { Sethi and Iqbal } \\
\text { 2008) }\end{array}$ & $\begin{array}{l}\text { The criteria for gate reviews of new product development projects are } \\
\text { formally established in your firm. } \\
\text { The criteria for gate reviews are formally used in your firm. } \\
\text { The criteria for gate reviews are strictly adhered to. }\end{array}$ \\
\hline $\begin{array}{l}\text { Objective } \\
\text { Criteriaa }^{a} \\
\text { (Existing } \\
\text { measure from } \\
\text { Sethi and Iqbal } \\
\text { 2008) }\end{array}$ & $\begin{array}{l}\text { The criteria for gate reviews are open to interpretation. (R) } \\
\text { The criteria for gate reviews vary depending on who does the reviewing. (R) } \\
\text { The criteria for gate reviews are open to negotiations. (R) }\end{array}$ \\
\hline $\begin{array}{l}\text { Entrepreneuria } \\
\text { V Innovative } \\
\text { Culture } \\
\text { (Adapted from } \\
\text { Deshpande, } \\
\text { Farley, and } \\
\text { Webster 1993) }\end{array}$ & $\begin{array}{l}\text { The glue that holds my business unit together is a commitment to } \\
\text { innovation and development. There is an emphasis on being first. } \\
\text { The head of my business unit is generally considered to be an } \\
\text { entrepreneur, an innovator, or a risk-taker. } \\
\text { My business unit is a very dynamic and entrepreneurial place. People are } \\
\text { willing to stick their necks out and take risks. }\end{array}$ \\
\hline $\begin{array}{l}\text { Politicization } \\
\text { of Gate } \\
\text { Reviews } \\
\text { (New measure } \\
\text { based on } \\
\text { Buchanan and } \\
\text { Badham 2003; } \\
\text { Fairholm 1993) }\end{array}$ & $\begin{array}{l}\text { Gate reviews are highly politicized. } \\
\text { Gate reviews are grounds for turf battles for functional managers. } \\
\text { Gate reviews are conducted with a sense of impartiality. (R) } \\
\text { During gate reviews, it is that mainly committee members' favourite } \\
\text { projects are supported. }\end{array}$ \\
\hline
\end{tabular}




\begin{tabular}{|c|c|}
\hline $\begin{array}{l}\text { Gate } \\
\text { Conditionality } \\
\text { (Existing }^{a} \\
\text { measure from } \\
\text { Sethi and Iqbal } \\
2008 \text { ) }\end{array}$ & $\begin{array}{l}\text { Gate reviews of projects allow a project to be approved even if all criteria are } \\
\text { not met, conditional on those criteria being met in the future. } \\
\text { The review criteria recognize that some projects follow a different } \\
\text { development sequence and allow such projects to proceed further even if they } \\
\text { only meet the criteria partially. } \\
\text { The review criteria recognize that sometimes various development activities } \\
\text { need to be approved out of sequence and allows for that to happen. }\end{array}$ \\
\hline $\begin{array}{l}\text { Demotivating } \\
\text { Gate } \\
\text { Experience } \\
\text { (New measure } \\
\text { based on } \\
\text { Kacmar et al. } \\
\text { 1999) }\end{array}$ & $\begin{array}{l}\text { The experience of gate reviews is demotivating. } \\
\text { Gate reviews are a great experience. (R) } \\
\text { The experience of gate reviews is satisfying. (R) }\end{array}$ \\
\hline $\begin{array}{l}\text { Market } \\
\text { Turbulence } \\
\text { (Existing }^{\text {Exing }} \\
\text { measure from } \\
\text { Jaworski and } \\
\text { Kohli 1993) }\end{array}$ & $\begin{array}{l}\text { It is difficult to predict how customers' needs and requirements will evolve } \\
\text { in our markets. } \\
\text { It is difficult to forecast competitive actions. } \\
\text { Generally, it is difficult to understand how the market will change. } \\
\text { There is a great deal of uncertainty in our markets. }\end{array}$ \\
\hline $\begin{array}{l}\text { Technological } \\
\text { Turbulence } \\
\text { (Existing } \\
\text { measure from } \\
\text { Jaworski and } \\
\text { Kohli 1993) }\end{array}$ & $\begin{array}{l}\text { The technology in our industry is changing rapidly. } \\
\text { Technological changes provide big opportunities in our industry. } \\
\text { A large number of new product ideas have been made possible through } \\
\text { technological breakthroughs in our industry. } \\
\text { Technological developments in our industry are rather minor. (R) }\end{array}$ \\
\hline $\begin{array}{l}\text { Innovativeness } \\
\text { of New } \\
\text { Products } \\
\text { New measure } \\
\text { based on Im and } \\
\text { Workman } 2004 ; \\
\text { Sarin and } \\
\text { McDermott } \\
2003 \text { ) }\end{array}$ & $\begin{array}{l}\text { To what extent did new products in your business unit: } \\
\text { Capture target markets that are new or different from our traditional markets. } \\
\text { Introduce innovative products compared with our traditional products. } \\
\text { Introduce breakthrough products that have taken our business unit in newer } \\
\text { directions. } \\
\text { Introduce products with the latest or cutting edge technologies. }\end{array}$ \\
\hline $\begin{array}{l}\text { Performance of } \\
\text { New Products } \\
\text { (Existing - Sethi } \\
\text { 2000) }\end{array}$ & $\begin{array}{l}\text { 1. Sales } \\
\text { 2. Market Share } \\
\text { 3. Profit } \\
\end{array}$ \\
\hline
\end{tabular}




\section{Appendix 2}

Item Level Tests for Outliers and Normality

\begin{tabular}{|c|c|c|c|c|}
\hline \multirow[t]{2}{*}{ Concept/Items } & \multicolumn{2}{|c|}{ Outliers ( $Z$ value) } & \multicolumn{2}{|c|}{ Normality } \\
\hline & Min. & Max. & Skewness & Kurtosis \\
\hline Pol.Org. Climate & & & & \\
\hline PolOrgCll & -2.46 & 1.36 & -.72 & -.11 \\
\hline PolOrgCl2 & -2.29 & 1.61 & -.50 & -.46 \\
\hline PolOrgCl3 (R) & -2.43 & 1.24 & -.74 & -.52 \\
\hline PolOrgCl4 & -1.96 & 1.68 & -.15 & -.95 \\
\hline PolOrgCl5 & -1.98 & 1.86 & -.02 & -.56 \\
\hline PolOrgC16 & -1.79 & 1.86 & .06 & -.86 \\
\hline PolOrgCl7 & -2.02 & 1.84 & -.16 & -.84 \\
\hline Slack & & & & \\
\hline Slack1 (R) & -1.36 & 2.93 & .42 & .52 \\
\hline Slack2 & -1.24 & 2.69 & .64 & .32 \\
\hline Slack3 & -1.40 & 2.18 & .40 & .66 \\
\hline Slack4 & -1.34 & 2.12 & .39 & .79 \\
\hline Centralization & & & & \\
\hline Cent 1 & -2.69 & 1.43 & -.56 & -.49 \\
\hline Cent 2 & -1.92 & 2.06 & .16 & -.95 \\
\hline Cent 3 & -1.72 & 2.08 & .05 & -.99 \\
\hline Cent 4 & -1.48 & 2.35 & .40 & -.76 \\
\hline Cent 5 & -1.63 & 2.27 & .37 & -.89 \\
\hline Cent 6 & -1.95 & 2.40 & .46 & -.62 \\
\hline Cent 7 & -2.48 & 2.14 & .10 & -.66 \\
\hline Cent 8 & -2.36 & 2.09 & .16 & -.70 \\
\hline Power & & & & \\
\hline Accumulation & & . & & \\
\hline PowAccul (R) & -1.60 & 2.05 & .06 & -1.06 \\
\hline PowAccu2 & -1.98 & 1.89 & -.03 & -.88 \\
\hline PowAccu3 (R) & -2.25 & 2.30 & .24 & -.60 \\
\hline PowAccu4 & -4.27 & 1.54 & -1.26 & 2.84 \\
\hline PowAccu5 (R) & -2.42 & 2.04 & .12 & -.77 \\
\hline PowAccu6 & -2.76 & 1.56 & -.60 & -.01 \\
\hline PowAccu 7 & -2.19 & 1.88 & -.25 & -.36 \\
\hline Strict Criteria & & & & \\
\hline StrictCrl & -2.02 & 1.27 & -.53 & -.87 \\
\hline StrictCr2 & -1.92 & 1.55 & -.21 & -1.02 \\
\hline StrictCr3 & -1.77 & 1.86 & -.19 & -1.04 \\
\hline Objective Criteria & & & & \\
\hline$\overline{\mathrm{ObjCr} 1(\mathrm{R})}$ & -1.69 & 2.33 & .46 & -.83 \\
\hline $\mathrm{ObjCr} 2(\mathrm{R})$ & -1.55 & 2.18 & .53 & -.63 \\
\hline $\mathrm{ObjCr} 3(\mathrm{R})$ & -2.20 & 1.66 & -.50 & -.62 \\
\hline
\end{tabular}




\begin{tabular}{|c|c|c|c|c|}
\hline & $\begin{array}{c}\text { Min. } \\
\text { Z value }\end{array}$ & $\begin{array}{c}\text { Max. } \\
\text { Z value }\end{array}$ & Skewness & Kurtosis \\
\hline $\begin{array}{l}\text { Entrepreneurial/ } \\
\text { Innovative Culture } \\
\text { InnovCull } \\
\text { InnovCul2 } \\
\text { InnovCul3 }\end{array}$ & $\begin{array}{l}-1.78 \\
-1.64 \\
-1.70\end{array}$ & $\begin{array}{l}2.14 \\
1.86 \\
2.27\end{array}$ & $\begin{array}{c}.17 \\
-.14 \\
.10\end{array}$ & $\begin{array}{c}-.72 \\
-1.09 \\
-.72\end{array}$ \\
\hline $\begin{array}{l}\text { Politicization of } \\
\text { Gate Reviews } \\
\text { PolGateRev1 } \\
\text { PolGateRev2 } \\
\text { PolGateRev3 } \\
\text { PolGateRev4 }\end{array}$ & $\begin{array}{l}-1.82 \\
-1.79 \\
-1.82 \\
-1.83\end{array}$ & $\begin{array}{l}1.82 \\
1.77 \\
2.37 \\
1.98\end{array}$ & $\begin{array}{l}-.15 \\
-.14 \\
.27 \\
-.01\end{array}$ & $\begin{array}{l}-.76 \\
-.98 \\
-.60 \\
-.91\end{array}$ \\
\hline $\begin{array}{l}\text { Gate } \\
\text { Conditionality } \\
\text { CondCr1 } \\
\text { CondCr2 } \\
\text { CondCr3 }\end{array}$ & $\begin{array}{l}-2.30 \\
-2.71 \\
-3.21\end{array}$ & $\begin{array}{l}1.59 \\
1.69 \\
1.64\end{array}$ & $\begin{array}{l}-.67 \\
-.65 \\
-.91\end{array}$ & $\begin{array}{c}-.23 \\
-.01 \\
.95\end{array}$ \\
\hline $\begin{array}{l}\text { Demotivating Gate } \\
\text { Experience } \\
\text { Demotexp1 } \\
\text { Demotexp2 (R) } \\
\text { Demotexp3 (R) }\end{array}$ & $\begin{array}{r}\cdot \\
-1.34 \\
-2.08 \\
-1.56\end{array}$ & $\begin{array}{l}3.35 \\
2.21 \\
2.87\end{array}$ & $\begin{array}{c}1.03 \\
-.00 \\
.30\end{array}$ & $\begin{array}{c}1.4 \\
-.30 \\
-.33\end{array}$ \\
\hline $\begin{array}{l}\text { Market } \\
\text { Turbulence } \\
\text { MktTurb1 } \\
\text { MktTurb2 } \\
\text { MktTurb3 } \\
\text { MktTurb4 }\end{array}$ & $\begin{array}{l}-2.07 \\
-2.21 \\
-2.19 \\
-2.45\end{array}$ & $\begin{array}{l}1.95 \\
2.09 \\
2.09 \\
1.70\end{array}$ & $\begin{array}{c}-.08 \\
-.11 \\
.05 \\
-.11\end{array}$ & $\begin{array}{l}-1.06 \\
-.84 \\
-.99 \\
-.82\end{array}$ \\
\hline $\begin{array}{l}\text { Technological } \\
\text { Turbulence } \\
\text { TechTurb1 } \\
\text { TechTurb2 } \\
\text { TechTurb3 } \\
\text { TechTurb4 (R) }\end{array}$ & $\begin{array}{l}-2.09 \\
-2.54 \\
-2.45 \\
-2.09\end{array}$ & $\begin{array}{l}1.48 \\
1.38 \\
1.58 \\
1.49\end{array}$ & $\begin{array}{l}-.15 \\
-.42 \\
-.18 \\
-.28\end{array}$ & $\begin{array}{c}-1.01 \\
-.75 \\
-.62 \\
-1.03\end{array}$ \\
\hline $\begin{array}{l}\text { Innovativeness of } \\
\text { New Products } \\
\text { Innov1 } \\
\text { Innov2 } \\
\text { Innov3 } \\
\text { Innov4 }\end{array}$ & $\begin{array}{r}-1.73 \\
-2.09 \\
-1.76 \\
-1.79\end{array}$ & $\begin{array}{l}2.15 \\
1.86 \\
2.27 \\
1.99\end{array}$ & $\begin{array}{r}-.15 \\
-.19 \\
-.06 \\
-.16\end{array}$ & $\begin{array}{l}-1.08 \\
-.67 \\
-.78 \\
-.93\end{array}$ \\
\hline $\begin{array}{l}\text { Performance of } \\
\text { New Products } \\
\text { Sales } \\
\text { Market Share } \\
\text { Profit }\end{array}$ & $\begin{array}{l}-2.20 \\
-2.29 \\
-1.99\end{array}$ & $\begin{array}{l}1.88 \\
1.88 \\
2.40\end{array}$ & $\begin{array}{l}-.37 \\
-.47 \\
-.25\end{array}$ & $\begin{array}{l}-.51 \\
-.10 \\
-.47\end{array}$ \\
\hline
\end{tabular}




\section{Appendix 3}

Difference in Responses between Groups

\begin{tabular}{|c|c|c|c|c|c|c|}
\hline \multirow[t]{2}{*}{ Concept } & \multicolumn{2}{|c|}{$\begin{array}{c}\text { Early vs. Late } \\
\text { Response (t -test) }\end{array}$} & \multicolumn{2}{|c|}{$\begin{array}{c}\text { Mail vs. Fax } \\
\text { Responses (t -test) }\end{array}$} & \multicolumn{2}{|c|}{$\begin{array}{c}\text { Respondents' } \\
\text { Background } \\
\text { (One way Anova) }^{\text {b }}\end{array}$} \\
\hline & $t$ & Significance & $t$ & Significance & $\mathbf{F}$ & Significance \\
\hline Pol. Org. Climate & -.73 & .47 & -.69 & .49 & .47 & .71 \\
\hline Slack & -1.53 & .13 & .89 & .38 & .10 & .96 \\
\hline Centralization & -1.60 & .11 & .32 & .75 & .24 & .87 \\
\hline Power Accum. & -.27 & .79 & -1.10 & .28 & .30 & .82 \\
\hline Strict Criteria & -1.87 & .06 & .32 & .75 & 1.70 & .17 \\
\hline Objective Criteria & .55 & .58 & 1.43 & .16 & .50 & .68 \\
\hline Innov/Ent Culture & -.24 & .81 & .44 & .66 & .10 & .96 \\
\hline Pol. Gate Atm. & .29 & .77 & -1.12 & .27 & .36 & .78 \\
\hline Gate Cond. & 1.39 & .17 & .54 & .59 & .61 & .61 \\
\hline $\begin{array}{l}\text { Demotivating } \\
\text { Gate Atmosphere }\end{array}$ & -1.23 & .22 & .39 & .70 & .18 & .91 \\
\hline Market Turb. & -1.79 & .08 & -.45 & .65 & .32 & .81 \\
\hline Tech Turbulence & -1.66 & .10 & .28 & .78 & .91 & .44 \\
\hline Innovativeness & -1.08 & .28 & 1.00 & .32 & .86 & .47 \\
\hline Performance & -.78 & .44 & -.38 & .70 & .10 & .96 \\
\hline
\end{tabular}

' Test of non-response bias (Armstrong and Overton 1977).

' Comparison of concept scores across respondents with different backgrounds (i.e., marketing, product development/R\&D, general management, manufacturing) 


\section{Appendix 4 \\ Qualitative Research Interview Protocol}

Purpose: The purpose of the interview is to understand your experience of navigating your new projects through the Stage-Gate process including the problems that are encountered. There are no right or wrong answers. So please freely report your own experiences. Your responses will be reported anonymously without identifying either you or your organization.

1. Respondent's Background

a) Your department?

b) Your title?

c) Is your department a part of a division in a large firm? (If yes, the following questions will focus on the division, if no, they will focus on the company).

2. Background of the Respondent's Division/Company and its Products

a) How large is your division/company in terms of number of employees, and annual sales?

b) Please describe the kinds of products your division/company makes.

c) What is your division's/company's/product line's ranking in your industry (e.g. in terms of market share)?

Respond to the following on $a 1$ to 7 scale $(1=$ Not at all and $7=A$ great deal $)$.

d) To what extent does your division/company emphasize innovation?

e) To what extent does it emphasize an entrepreneurial focus in innovation?

f) How volatile/changing are technologies in your industry?

g) How volatile/changing are your division's/company's markets?

h) How would you describe the structure of your division/company - is decision making centralized (i.e., concentrated in the hands of those at the top)?

i) Are there surplus resources available in your division/company?

j) To what extent does politics play a role in decision making in your division/company, in general? 
3. Company's Stage-Gate Process and Managers' Experience of Gate Reviews

a) Briefly describe the Stage-Gate process or its variant that your division/company uses (e.g. the number of stages and gates and their description).

b) Are there any challenges or problems associated with seeing new products through gate reviews that you have experienced?

\section{Nature of Gate Review Criteria}

1. Are the gate review criteria in your division/company formally laid out and strictly applied to all projects?

2. Are the gate review criteria objective in nature (i.e. not easily open to subjective interpretation)?

3. Are gate review criteria conditionally applied (i.e. if certain criteria are not met at a gate, the project is not held up but is allowed to move on to the next stage conditional on the criteria being met later)?

\section{Powerful Managers and Their Effect on Gate Reviews}

a) Do managers in your division/company who control important resources, processes, and agenda related to new products and thus have some power tend to influence the gate reviews or the fate of the products under review, thus politicizing gate reviews?

-If yes, in what way? Go to Part A (and choose the appropriate option from b to e)

-If no, what prevents these influences from coming into gate reviews? Go to Part B (and choose the appropriate option from $f$ to $i$ )

\section{Part A}

\begin{tabular}{|c|c|c|c|c|}
\hline 5a & 4a & 2d & 2e & Go to \\
\hline Yes & Yes & \multicolumn{2}{|c|}{ Yes in either } & Part A 5b \\
\hline Yes & No & \multicolumn{2}{|c|}{ Yes in either } & Part A 5c \\
\hline Yes & Yes & No & No & Part A 5d \\
\hline Yes & No & \multicolumn{2}{|c|}{ No in either } & Part A 5e \\
\hline
\end{tabular}

Go to b) if $5 a$ (power influence) is 'yes,' $4 a$ (strict and objective criteria) is 'yes', $2 d$ (innovation emphasis) and/or $2 e$ (entrepreneurial focus) are 'yes.' 
b) Do you think strict and objective criteria to some extent prevent the power influence from coming into gate reviews? Please explain.

- If the responses do not address the issue satisfactorily, then probe with: Survey results suggest that strict and objective criteria are not able to prevent powerful managers' influence from creeping into gate reviews. Why do you think this happens?

- If the innovation emphasis is 'yes,' ask: Does an innovation emphasis to some degree prevent power influence from coming into gate reviews?

- Please explain.

- If the entrepreneurial focus is 'yes' ask: Does an entrepreneurial focus to some degree prevent power influence from coming into gate reviews?

- Please explain

- If the responses to the above probes are not consistent with the empirical finding, then probe with: Survey results suggest that innovation emphasis and entrepreneurial focus do not prevent powerful managers' influence from creeping into gate reviews. Why do you think this happens?

\section{General}

Is there anything else you can think of that would prevent power influence from coming into gate reviews? (Go to 6 )

Go to $\mathrm{C}$ ), if 5 a (power influence) is 'pes,' 4 a (strict and objective criteria) is 'no,' $2 d$ (innovation emphasis) and/or $2 e$ (entrepreneur focus) are 'yes'.

c) Would strict and objective criteria have reduced the power influence from creeping in?

- Please explain.

- If the responses do not address the issue satisfactorily, then probe with: Survey results suggest that strict and objective criteria are not able to prevent powerful managers' influence from creeping into gate reviews. Why do you think this happens?

- If the innovation emphasis is 'yes,' ask: Does an innovation emphasis to some degree prevent power influence from coming into gate reviews?

- Please explain

- If the entrepreneurial focus is 'yes,' ask: Does an entrepreneurial focus to some degree prevent power influence from coming into gate reviews?

- Please explain. 
- If the responses to the above probes are not consistent with the empirical finding, then probe with: Survey results suggest that innovation emphasis and entrepreneurial focus do not prevent powerful managers' influence from creeping into gate reviews. Why do you think this happens?

\section{General}

Is there anything else you can think of that would prevent power influence from coming into gate reviews? (Go to 6)

Go to d) if 5 a (power influence) is 'yes', 4 a (strict and objective criteria) is 'yes', $2 d$ (innovation emphasis) and $2 e$ (entrepreneurial focus) are 'no'

d) Do you think strict and objective criteria to some extent prevent the power influence from coming into gate reviews?

- Please explain

- If the response does not address the above issue satisfactorily, then probe with:

Survey results suggest that strict and objective criteria are not able to prevent powerful managers' influence from creeping into gate reviews. Why do you think this happens?

Would an innovation emphasis and/or an entrepreneurial focus have affected the extent to which power influence makes gate reviews political?

- Please explain

- If the response to the above probe is not consistent with the empirical finding, then probe with: Survey results suggest that innovation emphasis and entrepreneurial focus do not prevent powerful managers' influence from creeping into gate reviews. Why do you think this happens?

\section{General}

Is there anything else you can think of that would prevent power influence from coming into gate reviews? (Go to 6)

Go to $e$ ) if $5 a$ (power influence) is 'yes', $4 a$ (strict and objective criteria) is 'no,' $2 d$ (innovation emphasis) and/or 2 (entrepreneurial focus) are "no"

e) Would strict and objective criteria have prevented to some extent power influence from creeping in?

- Please explain

- If the responses do not address the issue satisfactorily, then probe with: survey results suggest that strict and objective criteria are not able to prevent powerful managers' influence from creeping into gate reviews. Why do you think this happens? 
Would an innovation emphasis or an entrepreneurial focus have prevented power influence from coming into gate reviews?

- Please explain

- If the response to the above probe is not consistent with the empirical finding, then probe with: Survey results suggest that innovation emphasis and entrepreneurial focus do not prevent powerful managers' influence from creeping into gate reviews. Why do you think this happens? (Go to 6)

General

Is there anything else you can think of that would prevent power influence from coming into gate reviews?

Part B

\begin{tabular}{|c|c|c|c|c|}
\hline $5 \mathbf{a}$ & $4 a$ & $2 d$ & $\mathbf{2 e}$ & Go to \\
\hline No & Yes & \multicolumn{2}{|c|}{ Yes in either } & Part B 5f \\
\hline No & No & Yes & Yes & Part B 5g \\
\hline No & Yes & No & No & Part B 5h \\
\hline No & No & No & No & Part b 5i \\
\hline
\end{tabular}

Go to $f$ ) if $5 a$ (power influence) is 'no' and $4 a$ (strict and objective criteria) is 'yes', $2 d$ (innovation emphasis) and/or $2 e$ (entrepreneurial focus) are 'yes.'

f) Do you think the strict and objective criteria are responsible for the prevention of such power influence?

- Please explain

- If the responses do not address the issue satisfactorily, then probe with: Survey results suggest that strict and objective criteria are not able to prevent powerful managers' influence from creeping into gate reviews. Why do you think this happens?

Do you think an innovation emphasis is responsible for the prevention of such power influence?

- Please explain

Do you think an entrepreneurial focus is responsible for the prevention of such power influence?

- Please explain.

- If the response to the above probe is not consistent with the empirical finding, then probe with: Survey results suggest that innovation emphasis and entrepreneurial do 
not prevent powerful managers' influence from creeping into gate reviews. Why do you think this happens? (Go to 0

Go to g) if 5 a (power influence) is 'no' and $4 a$ (strict and objective criteria) is 'no', $2 d$ (innovation emphasis) and $2 e$ (entrepreneurial focus) are 'yes.'

g) Why doesn't power influence creep in when strict and objective criteria are absent? Please explain.

- If the response does not address the issue satisfactorily, then probe with: Survey results suggest that strict and objective criteria are not able to prevent powerful managers' influence from creeping into gate reviews. Why do you think this might be happening?

Do you think an innovation emphasis is responsible for the prevention of such power influence?

- Please explain.

Do you think an entrepreneurial focus is responsible for the prevention of such power influence?

- Please explain.

- If the response to the above probe is not consistent with the empirical finding, then probe with: Survey results suggest that innovation emphasis and entrepreneurial focus do not prevent powerful managers' influence from creeping into gate reviews. Why do you think this happens? (Go to 6)

Go to $h$ ) if 5 a (power influence) is 'no,' $4 a$ (strict and objective criteria) is 'yes,' $2 d$ (innovation emphasis) and $2 e$ (entrepreneurial focus) are 'no.'

h) Do you think strict and objective criteria are responsible for the prevention of power influence?

- Please explain

- If the responses do not address the issue satisfactorily, then probe with: Survey results suggest that strict and objective criteria are not able to prevent powerful managers' influence from creeping into gate reviews. Why do you think this happens?

Why doesn't power influence creep in when innovation emphasis and entrepreneurial focus are absent? Please explain.

- If the response to the above probe is not consistent with the empirical finding, then probe with: Survey results suggest that innovation emphasis and entrepreneurial focus do not prevent powerful managers' influence from creeping into gate reviews. Why do you think this happens? (Go to 6) 
Go to $i$ ) if $5 a$ (power influence) is 'no,' $4 a$ (strict and objective) is 'no,' $2 d$ (innovation emphasis) and 2 (entrepreneurial focus) are 'no.'

i) Why doesn't power influence creep in when strict and objective criteria are absent? Please explain.

- If the response does not address the issue satisfactorily, then probe with: Survey results suggest that strict and objective criteria are not able to prevent powerful managers' influence from creeping into gate reviews. Why do you think this happens?

Why doesn't power influence creep in when innovation emphasis and entrepreneurial focus are absent? Please explain.

- If the response to the above probe is not consistent with the empirical finding, then probe with: Survey results suggest that innovation emphasis and entrepreneurial focus do not prevent powerful managers' influence from creeping into gate reviews. Why do you think this happens?

Then what prevents power influence from creeping into the review process? (Go to 6)

\section{Politicization of Gate Reviews and Demotivation of Managers}

a) - Do politicized gate reviews (as a result of power influence) demotivate NPD managers?

- If yes, in what way? Go to Part A (and choose the appropriate option either b or c)

- If no, why do you think it doesn't happen? Go to Part B (and choose the appropriate option either $d$ or e)

\section{Part A}

Go to b), If 6a (demotivated managers) is 'yes' and 4 b (conditional criteria) is 'yes.'

b) Does conditional nature of gate criteria help reduce the extent of demotivation?

- Please explain

- If the response to the first probe (if yes, how?) is not satisfactory, then probe with:

Survey results suggest that conditional criteria do not reduce the demotivation of NPD managers. Why do you think this happens?

\section{General}

Are there any other negative effects of politicized gate reviews besides manager demotivation? (Go to 7) 
Go to c), if 6 a (demotivated managers) is 'yes' and $4 b$ (conditional criteria) is 'no.'

c) Would managers be less demotivated if the criteria were conditional?

- Please explain.

-If the response to the first probe (if yes, why?) is not satisfactory, then probe with: Survey results suggest that conditional criteria do not reduce the demotivation of NPD managers. Why do you think this happens?

General

Are there any other negative effects of politicized gate reviews besides manager demotivation? (Go to 7)

\section{Part B}

Go to d), If $6 a$ (demotivated managers) is 'no' and $4 \mathrm{~b}$ (conditional criteria) is 'yes.'

d) Does conditional nature of gate criteria prevent demotivation?

- Please explain

- If the response to the first probe (if yes, how?) is not satisfactory, then probe with: Survey results suggest that conditional criteria do not reduce the demotivation of NPD managers. Why do you think this happens?

Are there any other factors that prevent demotivation?

General

Are there any other negative effects of politicized gate reviews? (Go to 7)

Go to e), if 6a (demotivated managers) is 'no' and $4 \mathrm{~b}$ (conditional criteria) is 'no.'

e) Why are managers not demotivated given that the criteria are not conditional?

- Please explain.

- If the response is not satisfactory, then probe with: Survey results suggest that conditional criteria do not reduce the demotivation of NPD managers. Why do you think this happens?

Are there any other factors that prevent demotivation?

General

Are there any other negative effects of politicized gate reviews? (Go to 7) 
7. Product Managers' Motivation and Innovativeness of New Products

a) If managers feel demotivated, does it adversely affect the new products they develop, e.g., product innovativeness?

- If yes, in what way? Go to Part A (and choose appropriate option from b to d) - If no, why do you think it doesn't happen? Go to Part B (and choose appropriate option from e to $g$ )

\section{Part A}

\begin{tabular}{|c|c|c|c|}
\hline $\mathbf{7 a}$ & $\mathbf{2 f}$ & $\mathbf{2 g}$ & Go to \\
\hline Yes & Yes & Yes & Part A 7b \\
\hline Yes & No & Yes & Part A 7c \\
\hline Yes & Yes & No & Part A 7d \\
\hline
\end{tabular}

Go to b), If 7a (demotivation affects innovativeness) is 'yes,' $2 \mathrm{f}$ (volatile technology) is 'yes,' $2 \mathrm{~g}$ (volatile markets) is 'yes.'

b) Under conditions of volatile technology in your industry do demotivated managers develop less/more innovative products? Why?

Do demotivated managers develop less/more innovative products when the market is volatile? Why?

- If the response above is not consistent with the empirical finding, then probe with: Survey results suggest that market volatility improves the negative relationship between demotivation and innovativeness. Why do you think this happens?

\section{General}

Are there any other negative effects of manager demotivation? (Go to 8)

Go to $\mathrm{c}$ ), If 7 a (demotivation affects innovativeness) is 'yes,' $2 \mathrm{f}$ (volatile technology) is 'no,' $2 \mathrm{~g}$ (volatile markets) is 'yes.'

c) Would volatile technology in your industry have influenced whether demotivated managers develop less/more innovative products?

- Please explain.

Do demotivated managers develop less/more innovative products when the market is volatile?

- Please explain 
- If the response above is not consistent with the empirical finding, then probe with: Survey results suggest that market volatility improves the negative relationship between demotivation and innovativeness. Why do you think this happens?

\section{General}

Are there any other negative effects of manager demotivation? (Go to 8)

Go to d), If 7 a (demotivation affects innovativeness) is 'yes,' $2 \mathrm{f}$ (volatile technology) is 'yes,' $2 \mathrm{~g}$ (volatile markets) is 'no.'

d) Under conditions of volatile technology in your industry do demotivated managers develop less/more innovative products?

- Please explain

Would market volatility have influenced whether demotivated managers develop less/more innovative products? Why?

- If the response above is not consistent with the empirical finding, then probe with: Survey results suggest that market volatility improves the negative relationship between demotivation and innovativeness. Why do you think this happens?

\section{$\underline{\text { General }}$}

Are there any other negative effects of manager demotivation? (Go to 8)

\section{Part B}

\begin{tabular}{|c|c|c|c|}
\hline $\mathbf{7 a}$ & $\mathbf{2 f}$ & $\mathbf{2 g}$ & Go to \\
\hline No & Yes & Yes & Part B 7e \\
\hline No & No & Yes & Part A 7f \\
\hline No & No & No & Part A 7g \\
\hline
\end{tabular}

Go to e), If 7 a (demotivation affects innovativeness) is 'no,' $2 \mathrm{f}$ (volatile technology) is 'yes,' $2 \mathrm{~g}$ (volatile markets) is 'yes.'

e) Is volatile technology in your industry the reason that demotivated managers do not develop less innovative products?

- Please explain.

- Is market volatility the reason that demotivated managers do not develop less innovative products?

- Please explain. 
- If the response above is not consistent with the empirical finding, then probe with:

Survey results suggest that market volatility improves the negative relationship

between demotivation and innovativeness. Why do you think this happens?

\section{$\underline{\text { General }}$}

Are there any other negative effects of manager demotivation? (Go to 8)

Go to $\mathrm{f}$ ), If $7 \mathrm{a}$ (demotivation affects innovativeness) is 'no,' $2 \mathrm{f}$ (volatile technology) is 'no,' $2 \mathrm{~g}$ (volatile markets) is 'yes.'

f) If technologies were volatile in your industry, would managers' demotivation have affected product innovativeness?

- Please explain.

- Is market volatility the reason that demotivated managers do not develop less innovative products?

- Please explain.

- If the response above is not consistent with the empirical finding, then probe with: Survey results suggest that market volatility improves the negative relationship between demotivation and innovativeness. Why do you think this happens?

\section{$\underline{\text { General }}$}

Are there any other negative effects of manager demotivation? (Go to 8)

Go to $\mathrm{g}$ ), If 7 a (demotivation affects innovativeness) is 'no,' $2 \mathrm{f}$ (volatile technology) is 'no,' $2 \mathrm{~g}$ (volatile markets) is 'no.'

g) If technologies were volatile in your industry, would managers' demotivation have affected product innovativeness?

- Please explain.

If markets were volatile, would managers' demotivation have affected product innovativeness?

- Please explain

- If the response above is not consistent with the empirical finding, then probe with:

Survey results suggest that market volatility improves the negative relationship between demotivation and innovativeness. Why do you think this happens?

General

Are there any other negative effects of manager demotivation? (Go to 8) 


\section{Effect of Innovativeness on Performance of New Products}

a) Does innovativeness of your products influence their market performance?

- Please explain.

\section{Organizational Politics and Power Accumulation by Senior Managers}

a) Do you think politics creates a climate in which senior managers try to acquire power i.e. bring resources, products, operations, processes and agenda under their control?

- If yes, why? Go to Part A (choose appropriate option from $\mathrm{c}$ to f)

- If no, why not? Go to Part B (choose appropriate option from g to j)

\section{Part A}

\begin{tabular}{|c|c|c|c|}
\hline 9a (Pol Cl $\rightarrow$ PowAccum) & 2h (Cent) & 2i (Surplus) & Go to \\
\hline Yes & Yes & Yes & Part A, 9b \\
\hline Yes & No & Yes & Part A, 9c \\
\hline Yes & Yes & No & Part A, 9d \\
\hline Yes & No & No & Part A, 9e \\
\hline
\end{tabular}

Go to b), if $9 \mathrm{a}$ (senior managers acquire power) is 'yes,' $2 \mathrm{~h}$ (centralization) is 'yes,' $2 i$ (surplus resources) is ' $y e s$ '

b) Does centralized structure influence if senior managers acquire more/less power in a political atmosphere?

- Please explain.

Do surplus resources influence if senior managers strive for more/less power in a political atmosphere?

- Please explain

Go to c), if $9 \mathrm{a}$ (senior managers acquire power) is 'yes,' $2 \mathrm{~h}$ (centralization) is 'no,' $2 \mathrm{i}$ (surplus resources) is 'yes'

c) If the structure had been centralized, would it have made senior managers acquire more/less power in a political atmosphere?

- Please explain 
Do surplus resources influence if senior managers strive for more/less power in a political atmosphere?

- Please explain.

Go to d), if $9 \mathrm{a}$ (senior managers acquire power) is 'yes,' $2 \mathrm{~h}$ (centralization) is 'yes,' $2 \mathrm{i}$ (surplus resources) is ' $\mathrm{no}$ '

d) Does centralized structure influence if senior managers acquire more/less power in a political atmosphere?

- Please explain.

If surplus resources had been available, would it have made senior managers acquire more/less power in a political atmosphere?

- Please explain

Go to e), if 9a (senior managers acquire power) is 'yes,' $2 \mathrm{~h}$ (centralization) is 'no,' $2 \mathrm{i}$ (surplus resources) is 'no'

e) If the structure had been centralized, would it have made senior managers acquire more/less power in a political atmosphere?

- Please explain

If surplus resources had been available, would it have made senior managers acquire more/less power in a political atmosphere?

- Please explain

\section{Part B}

\begin{tabular}{|c|c|c|c|}
\hline 9a (Pol Cl $\rightarrow$ PowAccum) & 2h (Cent) & 2i (Surplus) & Go to \\
\hline No & Yes & Yes & Part B, 9f \\
\hline No & No & Yes & Part B, 9g \\
\hline No & Yes & No & Part B, 9h \\
\hline No & No & No & Part B, 9i \\
\hline
\end{tabular}

Ask $\mathrm{f}$ ), if 9a (senior managers acquire power) is 'no' and $2 \mathrm{~h}$ (centralization) is 'yes,' $2 \mathrm{i}$ (surplus resources) is ' $y e s$ '

f) Does centralization make senior managers acquire less power in a political climate? 
- Please explain

Do surplus resources make senior managers acquire less power in a political climate?

- Please explain

Ask g), if 9a (senior managers acquire power) is 'no' and $2 \mathrm{~h}$ (centralization) is 'no,' $2 i$ (surplus resources) is 'yes'

g) If there were centralization, would it have led to power acquisition by senior managers in a political climate?

- Please explain

Do surplus resources make senior managers acquire less power in a political climate?

- Please explain

Ask h), if 9a (senior managers acquire power) is 'no' and $2 \mathrm{~h}$ (centralization) is 'yes,' $2 i$ (surplus resources) is 'no.'

h) Does centralization make senior managers acquire less power in a political climate?

- Please explain

If there were surplus resources, would they have led to power acquisition by senior managers in a political climate?

- Please explain

Ask i), if 9a (senior managers acquire power) is 'no' and $2 \mathrm{~h}$ (centralization) is 'no,' $2 \mathrm{i}$ (surplus resources) is 'no.'

i) If there were centralization, would it have led to power acquisition by senior managers in a political climate?

- Please explain

- If there were surplus resources, would they have led to power acquisition by senior managers in a political climate?

- Please explain. 


\section{General}

Are there any other factors that would have led to power accumulation by senior managers?

Any other comments about the gate review process, how well it works, politicization of decisions, etc.

Thank you very much for all your help! 


\section{Appendix 5a}

\section{COMPCO - Interview Summary}

Respondent's title:

Products:

Enterprise Type:

Number of employees:

Annual sales:

Rank in the industry:

Innovation emphasis:

Entrepreneurial focus:

Technology turbulence:

Market Turbulence:

Centralization:

Slack:

Political Org. Climate:

Number of gates/reviews:

Strictness of the criteria:

Objectivity of the criteria:
Product Team Leader

Components for the Automotive Industry

Large

About 30,000

About $\$ 6$ billion

Number two in the industry

5 (on a 1 to 7 scale - where $1=$ not at all and $7=$ a great deal)

5 (on a 1 to 7 scale)

5 (on a 1 to 7 scale)

5 (on a 1 to 7 scale)

4 (on a 1 to 7 scale)

3 (on a 1 to 7 scale)

6 (on a 1 to 7 scale)

6

Moderate

Moderate

Moderate

\section{Feedback on the Model and Unconfirmed Effects}

Sub-Model 1: Effect of Political Organizational Climate on Power Accumulation (with Centralization and Slack as Moderators): According to this respondent, in the highly political organizational climate, senior managers try to accumulate power by acquiring resources.

Moderate centralization, he agreed, increases power accumulation in a political climate. The respondent said that low slack in his organization does not play a role in making senior managers accumulate more power. ${ }^{11}$ These responses are consistent with the hypothesized model and the results of the quantitative analysis.

\footnotetext{
11 This response is being considered consistent with the hypothesis because, implicit in the moderating hypothesis is that while high levels of slack will enhance the effect of political organizational climate on power accumulation, low levels of slack will not do so.
} 
The respondent was asked what would happen to power accumulation if slack were high; he said that he couldn't speculate.

Sub-Model 2: Effect of Power Accumulation on Politicization of Gate Reviews (with Strict and Objective Criteria and Entrepreneurial/Innovative Culture as Moderators): Powerful senior managers influence gate reviews thus making them political. This response is consistent with the hypothesized effect and the results of quantitative analysis. The respondent explained, "Senior managers see gate reviews as an opportunity to make their case and achieve their political ends. They may support a new product only if it is put under their control and made in their manufacturing facility," i.e. if it enhances their power.

Although gate review criteria are moderately strict and objective, politics and power still find their way into gate reviews. When asked to explain, the manager said, "Political interests find a way of getting into the review process, there is no stopping them, no matter how strict and objective the criteria." For similar reasons, an entrepreneurial/innovative culture also doesn't stop power accumulation from politicizing gate reviews. These responses are contrary to the hypothesized moderating effects but are consistent with the results of the quantitative analysis.

Sub-Model 3: Effect of Politicization of Gate Reviews on Demotivation of Managers (with Conditionality as a Moderator): As a result of the politicization of gate reviews, product development managers become demotivated. It doesn't matter if the criteria are moderately conditional.

When asked to explain, the respondent said, "The effect of the politicized reviews is quite forceful and conditionality doesn't make any difference." The response about the main effect of politicization is consistent with the hypothesis and the quantitative findings. On the other hand, the response about the moderating effect of conditionality is contradictory to the hypothesized effect but is consistent with the results of the quantitative analysis.

\section{Sub-Model 4: Effect of Demotivation on Innovativeness (with Technological and Market}

Turbulence as Moderators): Demotivation hurts the innovativeness of the new products developed by the organization. Volatile technology worsens the effect of demotivation on innovativeness. The respondent said, "Demotivated teams find it difficult to incorporate the latest technologies in the product and when they do so, they are sometimes late to the market, so it harms innovativeness." Thus, the responses about the main effect of demotivation and the moderating effect of technological turbulence are consistent with both the hypothesized effects and quantitative findings.

Also, when markets are volatile, products developed by demotivated teams are less innovative. This response is consistent with the hypothesis but not with the quantitative findings. The respondent said that market volatility basically occurs because customers change their requirements. He explained, "The products in our organization are usually made to specific customer requirements. Customers often say that they prefer products with new technologies. However, when it comes to actual implementation, customers sometimes can be quite risk averse and thus do not easily accept new technologies." This sometimes leads teams to reuse older technologies. While product development teams can look for an alternative acceptable new 
technology, their demotivation makes it difficult to identify and use such technologies - thus adversely affecting the innovativeness of new products.

When told that quantitative results suggest that market volatility makes it possible for even demotivated teams to develop innovative products, the manager expressed surprise. He said that this was not his experience.

Sub-Model 5: The Effect of Innovativeness on Performance: The respondent said that low innovativeness harms the market performance of new products. This is consistent with both the hypothesis and the quantitative findings.

\section{New Insights}

The model in this dissertation has been developed based on the assumption that new products are developed primarily based on the product development teams' view of what customer needs are. However, this interview suggests that sometimes new products are tailored to the customers' requirements (e.g., customer dictates the product's features, specifications, and technologies to be used). When products are developed based on customer requirements, political dynamics might be different at gate reviews. For example, it is likely to be more difficult to delay, demand major changes in, or kill a project for political reasons if specifications and design are driven by customer requirements. Future studies on organizational politics in new product innovation can focus on customized products to generate new insights.

This manager said that one major problem with the Stage-Gate process is that Stage-Gate, like Six-Sigma and House of Quality, assumes that you can capture customers' requirements at the beginning of the product development process and freeze them. However, customer requirements sometimes evolve over time. Product development teams have to learn.about these evolving requirements and incorporate them in the product. The Stage-Gate process in its current form does not leave room for such learning to take place. He said that the Stage-Gate process in his organization is being modified so that it permits learning to occur. The focus of the reviews is being changed from checking boxes to knowledge creation. Although some preliminary work on learning in the Stage-Gate process has been done (Hutchins and Muller 2012), more research on how gates can be made learning oriented needs to be conducted. 


\section{Appendix 5b}

\section{MACHCO - Interview Summary}

Respondent's title:

Product:

Number of employees:

Annual sales:

Rank in the industry:

Innovation emphasis:

Entrepreneurial focus:

Technology turbulence:

Market Turbulence:

Centralization:

Slack:

Political Org. Climate:

Number of gates/reviews:

Strictness of the criteria:

Objectivity of the criteria:

Conditionality of the criteria:
Product Development Leader

Precision Machine Tools

About 700

About $\$ 100$ million

Among top players

2 (on a 1 to 7 scale - where $1=$ not at all and $7=$ a great deal)

3 (on a 1 to 7 scale)

2 (on a 1 to 7 scale)

5 (on a 1 to 7 scale)

6 (on a 1 to 7 scale)

6 (on a 1 to 7 scale)

6 (on a 1 to 7 scale)

4

Low

Low

Moderate

\section{Feedback on the Model and Unconfirmed Effects}

\section{Sub-Model 1: Effect of Political Organizational Climate on Power Accumulation (with}

Centralization and Slack as Moderators): According to this respondent when the organizational climate is political as in his organization, managers try to acquire power through various means, e.g. by acquiring resources. The respondent felt that since his organization is highly centralized, senior managers try to acquire more power because it gives them a say in decision making. While enough slack is available, senior managers try to control resources when they are available and become more powerful. These responses are in agreement with the hypothesized model and the results of the quantitative analysis.

Sub-Model 2: Effect of Power Accumulation on Politicization of Gate Reviews (with Strict and Objective Criteria and Entrepreneurial/Innovative Culture as Moderators): Powerful senior managers do influence gate reviews in an attempt to maintain their power and so generally senior managers' favourite projects make it through the reviews but others may not. As a result, gate 
reviews get politicized. This response is consistent with both the quantitative findings and the hypothesis.

In this organization, gate review criteria are less strictly applied and do not influence the politicization of the reviews. Similarly, criteria objectivity is low and does not reduce the politicization of the gate reviews by powerful managers. The culture of organization is not so entrepreneurial/innovative and it doesn't influence the politicization of the reviews. These responses are consistent with both the quantitative findings and moderating hypotheses ${ }^{12}$.

The respondent felt that if the organization were to have an entrepreneurial/innovative culture, or more strict and objective criteria, it would reduce politicization of gate reviews but only to a limited extent. According to him, "Powerful managers are influential and their interference finds its way into gate reviews despite strict and objective criteria." Similarly, the interests of powerful managers are so strong that they are not easily deterred by an entrepreneurial and innovative culture.

\section{Sub-Model 3: Effect of Politicization of Gate Reviews on Demotivation of Managers (with} Conditionality as a Moderator): When gate reviews become politicized, product development managers become demotivated, which is consistent with the quantitative results and the hypothesis.

Conditional criteria do not help reduce demotivation. The respondent said that the influence of the politicization of gate reviews is so powerful in terms of demotivating product development managers that conditional criteria are not seen as any type of relief. This response is consistent with quantitative findings but contradicts the hypothesis.

\section{Sub-Model 4: Effect of Demotivation on Innovativeness (with Technological and Market}

Turbulence as Moderators): Demotivation adversely affects the innovativeness of new products. Technological turbulence for this organization is low and doesn't affect this relationship. These responses about the main effect of demotivation and the moderating effect of technology turbulence are consistent with the hypotheses and the findings of the quantitative analysis.

Market turbulence is high and it worsens the demotivation-innovativeness relationship which contradicts the quantitative results but is consistent with the hypothesis.

The respondent was told that his response about the effect of market turbulence contradicts the quantitative results, and was asked to explain this inconsistency. He said, "In MACHCO such a positive effect of market turbulence is not seen. When customer requirements change rapidly, demotivated teams often do not change their products in response. Thus, such products are not latest enough in satisfying customers' changed requirements," (i.e., they are not as innovative). He couldn't visualize a situation when the effect of market turbulence would be positive.

\footnotetext{
${ }^{12}$ These responses are being considered consistent with the hypotheses because, implicit in the moderating hypotheses is that while high levels of criteria strictness and objectivity and innovative culture will enhance the effect of power accumulation on politicization of reviews, low levels of these moderators will not do so.
} 
Sub-Model 5: The Effect of Innovativeness on Performance: The respondent said that low product innovativeness leads to lower market performance.

\section{New Insight}

The manager felt that the Stage-Gate process hampers the development of innovative new products. The Stage-Gate process is intended to minimize risk and so those projects make it through the gates that are less risky. Innovative new products, by their very nature, involve risk and so they usually don't make it through. Although some authors have suggested that the StageGate process does not favour innovative products that are risky in nature and does not adapt itself to unconventional product development that involves more uncertainty such as open innovation (Gronlund, Sjodin, and Frishammar 2010; Hutchins and Muller 2012), more research on how to make the Stage-Gate process responsive to risky or unconventional projects needs to be conducted. 


\section{Appendix 5c}

\section{FILTCO - Interview Summary}

Respondent's title:

Project Manager

Product:

Water filtration systems

Number of employees:

About 10,000

Annual sales:

About $\$ 3$ billion

Rank in the industry:

A major player in the industry

Innovation emphasis:

3 (on a 1 to 7 scale - where $1=$ not at all and $7=$ a great deal)

Entrepreneurial focus:

3 (on a 1 to 7 scale)

Technology turbulence:

3 (on a 1 to 7 scale)

Market Turbulence:

3 (on a 1 to 7 scale)

Centralization:

5 (on a 1 to 7 scale)

Slack:

3 (on a 1 to 7 scale)

Political Org. Climate:

6 (on a 1 to 7 scale)

Number of gates/reviews: 5

Strictness of the criteria: Low

Objectivity of the criteria: Low

Conditionality of the criteria: High

\section{Feedback on the Model and Unconfirmed Effects}

\section{Sub-Model 1: Effect of Political Organizational Climate on Power Accumulation (with}

Centralization and Slack as Moderators): The organizational climate is quite political and senior managers try to accumulate power. According to the respondent, centralization increases senior managers' attempts to get power in order to have some influence. Low slack does not affect the extent of power accumulation in the organization's political climate.

When asked what would happen if slack were high, the respondent said that managers would try to acquire more resources. All these responses are consistent with the main and moderating effect hypotheses and the findings of the quantitative analysis.

Sub-Model 2: Effect of Power Accumulation on Politicization of Gate Reviews (with Strict and Objective Criteria and Entrepreneurial/Innovative Culture as Moderators): Powerful senior managers influence project reviews and politicize them. This response is consistent with the quantitative findings and the hypothesis. 
Gate review criteria are less strict and objective and do not have any influence on the politicization of gate reviews by powerful managers. Similarly, the organizational culture is not so entrepreneurial/innovative and this has no effect. These responses are consistent with the results of the quantitative analysis and with the moderating hypotheses.

When asked what if the review criteria were more strict and objective and the culture more entrepreneurial/innovative, he suggested that they wouldn't make any difference because powerful managers would still exert their influence. "If someone senior and powerful really wants to get his project through he can do it."

\section{Sub-Model 3: Effect of Politicization of Gate Reviews on Demotivation of Managers (with}

Conditionality as a Moderator): Seeing all this politics at the reviews, there is demotivation. The respondent said that while it is good to see people coming to bat for their product, if they play politics and that demotivates others, it is not good. This response is consistent with both the quantitative results and the hypothesis.

Review criteria conditionality is high, but it doesn't make any difference in product development managers' demotivation. This response is consistent with the results of the quantitative analysis but not with the moderating hypothesis:

The respondent was asked why high conditionality doesn't reduce the level of demotivation. He said, "In my organization, even if the criteria are not met, there are no serious consequences and projects can still go through. This type of situation doesn't help reduce demotivation; instead sometimes it can have the opposite effect."

\section{Sub-Model 4: Effect of Demotivation on Innovativeness (with Technological and Market} Turbulence as Moderators): Demotivation clearly reduces innovativeness of products. Furthermore, since turbulence in technologies and markets is low, it does not play a role ${ }^{13}$. These responses are consistent with both the results of the quantitative analysis and the hypothesized main and moderating effects.

The respondent was asked if turbulence were high, would it have any effect. He responded that his organization operates in quite stable environments, so if there were to be more turbulence, initially the innovativeness of their products is likely to be adversely affected. However, he felt over a period of time, the organization would learn and adjust its behavior accordingly.

The respondent was informed that the results of the quantitative analysis suggest that market turbulence can help reduce the adverse effect of demotivation. When asked if he could comment why this was the case, he couldn't address this contradiction.

Sub-Model 5: The Effect of Innovativeness on Performance: According to the respondent, low innovativeness has a moderately negative effect on the market performance of products. This

\footnotetext{
${ }^{13}$ These responses are being considered consistent with the hypotheses because, implicit in the moderating hypotheses is that while high levels of technological and market turbulence will worsen the effect of demotivation on innovativeness, low levels of these moderators will not do so.
} 
response is consistent with both the hypothesis and the quantitative findings. The respondent said, "In our industry, true innovativeness is a rarity and customers do not seem to care about the bells and whistles you can put on a product."

\section{New Insight}

From this interview it emerged that if powerful senior managers want to get certain projects approved they will somehow get them approved, regardless of the rigor of the review criteria. For the projects they favour, senior managers can have project managers make promises at gates that the criteria will be successfully met. Subsequently, when those projects don't fulfill promises, these senior managers will intervene and protect project managers from any consequences. Researchers need to examine what happens when there are no serious consequences of not keeping the promises made at gate reviews. 


\section{Appendix 5d}

\section{ELECMACO - Interview Summary}

Respondent's title:

Product

Number of employees:

Annual sales:

Rank in the industry:

Innovation emphasis:

Entrepreneurial focus:

Technology turbulence:

Market Turbulence:

Centralization:

Slack:

Political Org. Climate:

Number of gates/reviews:

Strictness of the criteria:

Objectivity of the criteria:
Project Leader Design Group

Electronic Machines

About 18,000

About $\$ 10$ billion

Near the top of the industry

7 (on a 1 to 7 scale - where $1=$ not at all and $7=$ a great deal)

6 (on a 1 to 7 scale)

6 (on a 1 to 7 scale)

3 (on a 1 to 7 scale)

6 (on a 1 to 7 scale)

5 (on a 1 to 7 scale)

6 (on a 1 to 7 scale)

5

Low

Low

Low

\section{Feedback on the Model and Unconfirmed Effects}

Sub-Model 1: Effect of Political Organizational Climate on Power Accumulation (with Centralization and Slack as Moderators): The respondent believed that all organizations have some level of politics, and the climate in his organization is quite political. In such a political climate, senior managers do acquire power. However, he saw a range of seniority in these managers. Some of them are really top level managers, while others are more toward the middlelevel of seniority.

He said, "When the organization is more centralized, there is more power acquisition in a political climate. When decision making is in the hands of top level managers, it is the managers in the middle-level who try to accumulate more power and they are driven by their insecurity." This response about the main effect of a political climate and the moderating effect of centralization is consistent with the hypotheses and the quantitative findings. 
However, the respondent said that when there is a lot of slack, power accumulation doesn't occur. This response contradicts both the hypothesized moderating effect and the results of the quantitative analysis. The respondent explained, "When the organization is doing well and is profitable and there are surplus resources available, the middle-level managers feel less insecure and thus try less to acquire power." When asked to explain why the quantitative analysis produced an opposite effect of slack, the respondent said that this was not his experience.

Sub-Model 2: Effect of Power Accumulation on Politicization of Gate Reviews (with Strict and Objective Criteria and Entrepreneurial/Innovative Culture as Moderators): He felt that powerful managers do influence gate reviews a great deal in order to retain their hold on power and less strict and objective criteria do not make any difference. These responses are consistent with the quantitative findings and the hypotheses.

He was asked if the criteria were more strict and objective, would they reduce politicization. $\mathrm{He}$ responded that they still would not keep politics and power influence out of gate reviews. He said, "Senior managers including more middle-level managers find a way of influencing the gate reviews no matter how strict and objective the review criteria may be."

An entrepreneurial/innovative culture is not able to keep power influence out of gate reviews. This is consistent with the results of the quantitative analysis but not with the moderating hypothesis.

Sub-Model 3: Effect of Politicization of Gate Reviews on Demotivation of Managers (with Conditionality as a Moderator): As a result of the politicization of gate reviews, product development managers become cynical and demotivated. This response is consistent with both the hypothesis and the findings of the quantitative analysis.

However, once again, this respondent provided a nuanced response. He said, "The extent of demotivation among product development managers varies depending on their background. The engineers and technical experts are usually less demotivated than managers from non-technical backgrounds. If a project runs into problems at gate reviews or even gets cancelled, technical managers view it as a learning experience and feel that they can use the knowledge gained here in another project. It is the non-technical people who get more demotivated when there is a setback on a project during the reviews."

Conditionality of the criteria is low and it doesn't make any difference in how much product development managers get demotivated due to the politicization of gate reviews. This response is consistent with the findings of the quantitative analysis.

The respondent was asked if conditionality were high, would it help reduce demotivation. He explained, "Conditional criteria don't affect technical managers because their source of motivation is learning about new technology. For non-technical managers, conditional criteria do not make much difference because the negative effect of political gate reviews can be quite overwhelming for them."

Sub-Model 4: Effect of Demotivation on Innovativeness (with Technological and Market Turbulence as Moderators): Demotivation, this respondent agreed, lowered product 
innovativeness. This response is consistent with the results of the quantitative analysis and the hypothesis.

His view was that technological change throws open new opportunities for innovation and thereby weakens the adverse effect of demotivation. However, this contradicts the quantitative findings and the corresponding hypothesis.

When told about this contradiction, the respondent explained, "Non-technical managers usually see technology turbulence as more of a problem and threat than technical managers - which can worsen the effect of demotivation on innovativeness. If the project teams in an organization have more non-technical members, the overall effect of technological volatility can be negative."

Market turbulence is low and thus doesn't affect the demotivation-innovativeness relationship, which is consistent with the results of quantitative analysis and the moderating hypothesis. When asked, he couldn't say what would happen if market turbulence were high.

Sub-Model 5: The Effect of Innovativeness on Performance: The respondent said that low innovativeness has an adverse effect on the market performance of new products. This is consistent with both the hypothesis and the quantitative findings.

\section{New Insights}

One of the new insights provided by this respondent is that there is a range of senior managers some are top level managers and others are more middle-level managers. Some of the middlelevel managers tend to accumulate more power. The motivation of these middle managers is to make their position safe in the organization rather than acquire more control and influence.

The other insight that emerged was related to differences in views and behaviors of technical and non-technical managers in product development teams. This difference, for example, can explain why technical managers are more likely to view technological turbulence as an opportunity.

Future studies may want to take this distinction into account to better capture the organizational reality. 


\section{Appendix 5e}

\section{FABRICO - Interview Summary}

Respondent's title:

Product:

Number of employees:

Annual sales:

Rank in the industry:

Innovation emphasis:

Entrepreneurial focus:

Technology turbulence:

Market Turbulence:

Centralization:

Slack:

Political Org. Climate:

Number of gates/reviews:

Strictness of the criteria:

Objectivity of the criteria: Low

Conditionality of the criteria: Low

5

Low
Project Manager

Fabricated assemblies for commercial use

About 3,000

Not disclosed

Among top five

3 (on a 1 to 7 scale - where $1=$ not at all and $7=$ a great deal)

3 (on a 1 to 7 scale)

3 (on a 1 to 7 scale)

3 (on a 1 to 7 scale)

5 (on a 1 to 7 scale)

3 (on a 1 to 7 scale)

6 (on a 1 to 7 scale)

\section{Feedback on the Model and Unconfirmed Effects}

\section{Sub-Model 1: Effect of Political Organizational Climate on Power Accumulation (with}

Centralization and Slack as Moderators): The respondent said, "The climate in our organization is quite political and senior managers try to become powerful by controlling more physical and human resources, whenever they get the opportunity." Centralization is fairly high and it leads to greater power accumulation by senior managers in this political climate. This is senior managers' way of gaining influence. These responses are consistent with both the quantitative results and the hypotheses.

Slack is low and so it doesn't affect the level of power accumulation. This too is consistent with both the findings of quantitative analysis and the moderating hypothesis. The respondent couldn't say what would happen if slack were high.

Sub-Model 2: Effect of Power Accumulation on Politicization of Gate Reviews (with Strict and Objective Criteria and Entrepreneurial/Innovative Culture as Moderators): Powerful senior 
managers do politicize gate reviews. The respondent said, "If a project benefits another senior manager and is likely to increase his/her prestige or reputation, other senior managers try to shoot it down."

Strictness and objectivity of review criteria are quite low in this organization, and thus the criteria don't play a role in influencing the politicization of gate reviews under this environment of high power accumulation. The organization's culture is not so entrepreneurial and innovative and it doesn't affect the politicization of gate reviews. All the above responses are consistent with both the quantitative findings and the moderating hypotheses.

The respondent was asked if the criteria were strict and objective and the organization's culture were more entrepreneurial/innovative, would they be able to reduce the adverse effect of power accumulation on politicization of gate reviews. He said, "Senior managers have their own agendas that they want to pursue and strict and objective criteria and an innovative culture is ignored in this pursuit. Senior managers can always find problems with a project and couch their political intentions in objective terms to create problems for the project they do not want to support."

\section{Sub-Model 3: Effect of Politicization of Gate Reviews on Demotivation of Managers (with} Conditionality as a Moderator): Politicization of gate reviews demotivates product development managers. However, the respondent added, "If the setback to the project is not severe or fatal, product development teams recover their motivation quickly because they are experienced enough to know that in organizations there will always be politics and setbacks. If there is a more serious obstacle to the project, then demotivation can last longer." Conditionality of criteria is quite low and it does not play any role in influencing demotivation when politicization of reviews is quite high. These responses are consistent with both the quantitative findings and the hypotheses.

\section{Sub-Model 4: Effect of Demotivation on Innovativeness (with Technological and Market}

Turbulence as Moderators): Demotivation, the respondent said, reduces the innovativeness of new products developed by the firm. This response is consistent with the hypothesis and quantitative results.

Technological turbulence is low in this industry, so it has no effect on innovativeness when product development managers are demotivated. This response is consistent with the hypothesis and the quantitative findings.

When asked what would happen if technological turbulence were high, the respondent said its effect could be positive or negative. Engineers and technical people on teams, he felt, look at technological change with excitement and consider it a challenge and this may help energize them and make them develop innovative, cutting edge new products. But the real technical people are few in teams and the other non-technical people who are already demotivated, may get further demotivated when they encounter technological turbulence. So the overall effect of technological turbulence can be negative. 
Market turbulence is low, so it has no effect on the innovativeness of new products developed by demotivated managers. The respondent was asked what would happen if market turbulence were high. He said, "When market turbulence is high, new competitors are coming into the industry and customers demand newer things in the product, it can be seen as a challenge by all the members of the team. Market turbulence can create an external threat or challenge which has to be overcome. Such a challenge can unify, reenergize, and spur the team to do good work even though they may have been demotivated earlier." Thus, market turbulence can lead to more innovative new products when product development teams are demotivated. This is consistent with the results of quantitative analysis but not with the moderating hypothesis. In effect, this respondent explained why quantitative analysis has shown opposite effects of market and technological turbulence.

Sub-Model 5: The Effect of Innovativeness on Performance: The respondent said that low innovativeness negatively influences the performance of their products. This is consistent with both the hypothesis and the quantitative findings.

\section{New Insight}

This interview provided an explanation for the positive moderating effect of market turbulence and the negative moderating effect of technological turbulence on the DemotivationInnovativeness relationship. Market turbulence is likely to reenergize the entire team and help it work toward producing innovative outcomes. However, as the respondent explained, this explanation does not apply to technological turbulence, which is likely to energize mainly the technical experts or engineers on teams. The non-technical members may not feel as energized by technological turbulence. On the contrary, these demotivated non-technical members can become even less enthusiastic when they encounter technological turbulence which they may view as a problem. Often, non-technical members tend to outnumber the technical members on teams and thus on balance demotivated teams tend to become further frustrated by technological turbulence - which leads to less innovative products. 


\section{Appendix 5f}

\section{SOFTCO - Interview Summary}

Respondent's title:

Product:

Number of employees:

Annual sales:

Rank in the industry:

Innovation emphasis:

Entrepreneurial focus:

Technology turbulence:

Market Turbulence:

Centralization:

Slack:

Political Org. Climate:

Number of gates/reviews:

Strictness of the criteria:

Objectivity of the criteria:

Conditionality of the criteria:
Director - Product Development

Software

About 120

About $\$ 20$ million

Among top performers in its niche

7 (on a 1 to 7 scale - where $1=$ not at all and $7=$ a great deal)

7 (on a 1 to 7 scale)

7 (on a 1 to 7 scale)

7 (on a 1 to 7 scale)

7 (on a 1 to 7 scale)

6 (on a 1 to 7 scale)

6 (on a 1 to 7 scale)

3

High

High

Low

\section{Feedback on the Model and Unexpected Effects}

Sub-Model 1: Effect of Political Climate on Power Accumulation (with Centralization and Slack as Moderators): The respondent said that the climate in his organization is highly political and in this climate senior managers accumulate power. Centralization increases power accumulation in the political environment of the organization. Slack too, he felt, increases power accumulation when politics abounds. These responses are consistent with the main and moderating hypotheses and with the results of the quantitative analysis.

Sub-Model 2: Effect of Power Accumulation on Politicization of Gate Reviews (with Strict and Objective Criteria and Entrepreneuria/Innovative Culture as Moderators): Powerful managers influence gate reviews moderately and thus make them political, which is consistent with the quantitative results and the hypothesis.

When asked if strict and objective review criteria prevent the gate reviews from getting politicized, the respondent disagreed. He said, "When senior managers are really against a 
project, they somehow find a way to create problems for it and vice versa," i.e., if they are in favour, they get the project through - though he felt they don't do so very often. These responses are consistent with the findings of the quantitative analysis but contradict the hypotheses.

However, the respondent felt that the organization's strong focus on developing innovations prevents gate reviews from getting highly politicized, which is not consistent with the quantitative results but supports the hypothesis.

When told that the findings of the survey suggest that an entrepreneurial/innovative emphasis does not reduce politicization, he said, "In our case, it is not just our organization, but the entire industry is strongly focused on innovation, and that probably makes some difference."

\section{Sub-Model 3: Effect of Politicization of Gate Reviews on Demotivation of Managers (with} Conditionality as a Moderator): This moderate politicization of gate reviews demotivates product development managers moderately. Since conditionality is low, it does not influence the adverse effect of politicization. These responses are consistent with the hypotheses and the results of quantitative analysis.

However, when asked why politicization of gate reviews influences demotivation moderately, the respondent said, "Some of the project leaders are highly entrepreneurial and resourceful and find ways to go around project reviews to meet their requirements. If gate reviewers object to the purchase of some new equipment, the project leader will lease the equipment from a supplier and do away with the need for seeking approval for the purchase." Such actions generally keep teams' morale high.

\section{Sub-Model 4: Effect of Demotivation on Innovativeness (with Technological and Market}

Turbulence as Moderators): The respondent said that the moderate level of demotivation has a moderately adverse influence on the innovativeness of new products. He also suggested that technological volatility is good for innovativeness. The response about the main effect of demotivation is consistent with both the quantitative findings and hypothesis. However, the response related to the effect of technological turbulence contradicts both quantitative findings and the moderator hypothesis.

He was told that the quantitative results suggest that technological volatility harms innovativeness in teams with moderate motivation. He remarked, "Entrenched or well established organizations can view technological volatility as a problem, but newer set ups in fast moving industries like ours will generally see it as an opportunity to be more creative."

This respondent also said that market volatility helps in the development of more innovative products even if teams' morale is not so high. He said, "If markets and customer requirements change quickly, they create opportunities to come up with creative new products. These opportunities also create excitement in the team to effectively exploit them." Thus, this response is consistent with the results of quantitative analysis but not with hypothesized effect of market turbulence.

Sub-Model 5: The Effect of Innovativeness on Performance: The respondent said that moderately innovative products lead to moderate market performance - though innovative products may take 
some time to show solid profitability. This is consistent with both the hypothesis and the quantitative findings.

\section{New Insights}

It emerged that it is not just the innovative culture of the organization that matters, but also the innovation focus of the entire industry which influences how much power affects politicization of gate reviews. This industry level innovation focus will be an important factor to consider in future studies.

It also appears that project leaders can play an important role in minimizing the adverse effect of power and politics. For example, as noted earlier, an entrepreneurial and resourceful leader can obtain the equipment required for a project through other means and thus go around some of the obstacles created at gate reviews for obtaining that equipment - which helps keep the project team's morale high. This dissertation is an organizational level study, but future project level research can take this factor into account. 


\section{Appendix 5g}

\section{SEMCO - Interview Summary}

Respondent's title:

Senior Project Engineer

Product:

Semiconductors

Number of employees:

About 20,000

Annual sales:

About $\$ 8$ billion

Rank in the industry:

Among the top players in the industry

Innovation emphasis:

6 (on a 1 to 7 scale - where $1=$ not at all and $7=$ a great deal)

Entrepreneurial focus:

5 (on a 1 to 7 scale)

Technology turbulence:

7 (on a 1 to 7 scale)

Market Turbulence:

6 (on a 1 to 7 scale)

Centralization:

3 (on a 1 to 7 scale)

Slack:

2 (on a 1 to 7 scale)

Political Org. Climate:

4 (on a 1 to 7 scale)

Number of gates/reviews: $\quad 3$

Strictness of the criteria: Low

Objectivity of the criteria: Low

Conditionality of the criteria: Moderate

\section{Feedback on the Model and Unconfirmed Effects}

Sub-Model 1: Effect of Political Climate on Power Accumulation (with Centralization and Slack as Moderators): The respondent said that the climate in the organization is moderately political and that politics is an integral part of organizational life. This moderate level of politics leads to moderate power accumulation. He said that the low levels of centralization and slack in his organization don't play any role in influencing power accumulation in a political climate. These responses are consistent with the main and moderating effect hypotheses and with the findings of the quantitative analysis.

When told that the quantitative results show that high levels of centralization and slack increase the tendency to accumulate power in a political climate, this respondent felt that his experience doesn't suggest this. He said, "The tendency to accumulate power comes from seeing politics everywhere in the firm and slack and centralization shouldn't make any difference." 
Sub-Model 2: Effect of Power Accumulation on Politicization of Gate Reviews (with Strict and Objective Criteria and Entrepreneurial/Innovative Culture as Moderators): To some extent, senior managers in this organization use their power to influence gate reviews. Criteria strictness and objectivity are low and, as expected, don't influence the effect of power accumulation on the politicization of reviews. These responses are consistent with both the quantitative findings and the hypotheses.

On other hand, the organization's culture is entrepreneurial and innovative, but it doesn't influence the power accumulation-politicization relationship. This response is consistent with the quantitative findings but contradicts the moderating hypothesis.

This respondent said that even if the firm were to use more strict and objective review criteria, it would not prevent powerful managers' influence from coming into gate reviews. He explained, "If senior managers want to find fault with a project in order to scuttle it, they can find ways to do so. They can cast doubt on your ROI figures, that they are unrealistic and cannot be achieved." For a similar reason, the manager felt that even if the organization's culture were more entrepreneurial/innovative, it wouldn't keep power influence out.

Sub-Model 3: Effect of Politicization of Gate Reviews on Demotivation of Managers (with Conditionality as a Moderator): Moderate politicization of reviews to some extent demotivates managers. He said, "For some experienced product development managers though this demotivation does not last long because they accept political meddling as a part of organizational reality. But, if their projects are repeatedly rejected, even these managers can get demotivated." This response is consistent with both the quantitative findings and the hypothesis.

Furthermore, the respondent indicated that conditional review criteria are not able to reduce the extent of demotivation. He felt something more effective is needed to overcome demotivation such as product development managers taking political influence in their stride and thus not getting frustrated. This response is consistent with the findings of the quantitative analysis but not with the moderating hypothesis.

\section{Sub-Model 4: Effect of Demotivation on Innovativeness (with Technological and Market}

Turbulence as Moderators): The moderate level of demotivation in the organization somewhat reduces innovativeness. This is consistent with both the quantitative findings and the hypothesis.

The respondent explained that product development managers who take politics in their stride and thus are less demotivated do not have a negative influence on innovativeness.

Furthermore, he said, "If there is technological volatility, demotivated product development teams are likely to be overwhelmed by the resulting uncertainty and confusion and thus will find it difficult to develop innovative new products." This response is consistent with the moderating hypothesis and with the quantitative results.

He felt that market turbulence should worsen innovativeness similar to the effect of technological turbulence, which is consistent with the hypothesis but not with the quantitative results. 
When told that this response contradicts the quantitative findings, the respondent said, "It is possible that those product development managers who are less demotivated, because they take political influence in their stride, might be able to use market volatility as an opportunity and thus have a positive effect on innovativeness."

Sub-Model 5: The Effect of Innovativeness on Performance: A moderate level of innovativeness leads to moderate performance. However, the effect of innovativeness may be limited sometimes, because there are other factors affecting performance. This response is consistent with the hypothesis and the quantitative results.

\section{New Insights}

In this interview, it emerged that politicization of gate reviews does not demotivate all product development managers equally. For some experienced project managers this demotivation may not last long because they tend to accept political meddling as a part of organizational reality. While this factor cannot be taken into account in this dissertation that focuses on the organizational level, it can be taken into consideration in future project level studies.

Second, this interview generated an explanation for the positive moderating effect of market turbulence that was found in the quantitative analysis. Product development managers who are less demotivated, because they accept political influence as a part of organizational life, might be able to use market volatility as an opportunity and thus come up with more creative products. 


\section{Appendix 5h}

\section{COMAPPCO - Interview Summary}

Respondent's title:

Product:

Number of employees:

Annual sales:

Rank in the industry:

Innovation emphasis:

Entrepreneurial focus:

Technology turbulence:

Market Turbulence:

Centralization:

Slack:

Political Org. Climate:

Number of gates/reviews:

Strictness of the criteria:

Objectivity of the criteria:

Conditionality of the criteria:
Senior Product Development Engineer

Commercial appliance controls

About 350

About $\$ 100$ million

Leader in the industry

3 (on a 1 to 7 scale - where $1=$ not at all and $7=$ a great deal)

3 (on a 1 to 7 scale)

4 (on a 1 to 7 scale)

3 (on a 1 to 7 scale)

3 (on a 1 to 7 scale)

3 (on a 1 to 7 scale)

4 (on a 1 to 7 scale)

3

High

High

Low

\section{Feedback on the Model and Unconfirmed Effects}

Sub-Model 1: Effect of Political Climate on Power Accumulation (with Centralization and Slack as Moderators): The respondent said that the organizational climate is moderately political and it leads to a moderate level of power accumulation by senior managers. Centralization is low and it doesn't play a role in influencing power accumulation. Similarly, slack is low and doesn't have any influence. These responses are consistent with the hypotheses and with quantitative results.

She was asked if centralization and slack were high in the organization, would they influence the level of power accumulation in the moderately political organizational climate. She said, "High slack and centralization do not make a difference because my organization is not so political and the desire to gather power is not so high. A majority of our projects involve product upgrades and modifications, and there are only a few new products. Senior managers are less interested in these product upgrade projects because control over them doesn't bring any major advantage." 
Sub-Model 2: Effect of Power Accumulation on Politicization of Gate Reviews (with Strict and Objective Criteria and Entrepreneurial/Innovative Culture as Moderators): The respondent said that power accumulation by senior managers is at a moderate level and it politicizes gate reviews to a limited extent. This response is inconsistent with both the quantitative findings and the hypothesis.

Strict and objective criteria do not influence the effect of power accumulation on politicization. This response is consistent with the quantitative results but not with the hypothesis. The respondent explained, 'It isn't the case that only powerful senior managers' favourite projects are approved and others are not. Control over product upgrades doesn't appear to be so important for senior managers."

The culture of the organization is not entrepreneurial/innovative and it doesn't affect the politicization of gate reviews by powerful managers. This response supports both the results of quantitative analysis and the hypothesis.

Sub-Model 3: Effect of Politicization of Gate Reviews on Demotivation of Managers (with Conditionality as a Moderator): As gate reviews are not so politicized, it has only limited influence on product development managers' demotivation. Since conditionality of the review criteria is low, it doesn't play a role in affecting managers' demotivation. These responses are consistent with both the quantitative results and hypotheses.

Sub-Model 4: Effect of Demotivation on Innovativeness (with Technological and Market Turbulence as Moderators): Since there is low demotivation, it does not adversely affect the innovativeness of products much - which is consistent with the hypothesis and quantitative results.

Also, technological turbulence is moderate, and it helps improve innovativeness. The respondent said, "In my organization, there are more rapid changes in manufacturing technologies than product technologies. Product development teams are keen to learn about these changes in manufacturing technologies and find the learning process fun. This increases the teams' enthusiasm and gives an impetus to innovativeness." This response contradicts both the results of the quantitative analysis and the hypothesis.

Since market volatility is low, as expected, it does not have any effect on the demotivationinnovativeness relationship. This response is consistent with both the quantitative findings and the moderating hypothesis.

Sub-Model 5: The Effect of Innovativeness on Performance: The respondent explained that innovative product upgrades do lead to improved market performance - which is consistent with the hypothesis and quantitative results. 


\section{New Insights}

It emerged from this interview that the exercise of political and power influence depends on whether the new product is just an upgrade of an existing product or a new product. As this respondent said, senior managers are less interested in product upgrades because they don't bring them any major benefit. However, this is a project level issue and future project level studies on politics and innovation may want to take this factor into account.

Technological turbulence does not demotivate or reduce product innovativeness. Instead technical people enjoy technological changes, see them as an opportunity to learn and do new and fun things and produce new products. The manager felt that it is the attitude toward turbulence that matters. Turbulence can be seen as an opportunity or as a problem. When it is viewed as an opportunity it can positively impact innovativeness. On the other hand, those who view it as change and a disruption in their normal ways of doing things will find it a problem and it will adversely impact outcomes. 


\section{Appendix 5i}

\section{INDAUTCO - Interview Summary}

Respondent's title:

Product:

Number of employees:

Annual sales:

Rank in the industry:

Innovation emphasis:

Entrepreneurial focus:

Technology turbulence:

Market Turbulence:

Centralization:

Slack:

Political Org. Climate:

Number of gates/reviews:

Strictness of the criteria:

Objectivity of the criteria:

Conditionality of the criteria: Low

5

High

High
Project Manager

Industrial automation

About 150

About $\$ 40$ million

A major player in their niche

7 (on a 1 to 7 scale - where $1=$ not at all and $7=$ a great deal)

7 (on a 1 to 7 scale)

7 (on a 1 to 7 scale)

7 (on a 1 to 7 scale)

5 (on a 1 to 7 scale)

7 (on a 1 to 7 scale)

3 (on a 1 to 7 scale)

\section{Feedback on the Model and Unconfirmed Effects}

Sub-Model 1: Effect of Political Climate on Power Accumulation (with Centralization and Slack as Moderators): The respondent said that the organizational climate is less political and it doesn't lead to much power acquisition by senior managers. This response is consistent with the hypothesis and the quantitative findings.

The organization is centralized but this doesn't make managers acquire more power in this organization with low politics. Similarly, although slack is high, it doesn't lead to more power accumulation because the climate is less political and power accumulation is low. These responses are not consistent with the quantitative results and the moderating hypotheses ${ }^{14}$.

\footnotetext{
${ }^{14}$ These responses are being considered inconsistent with the moderating hypotheses because implicit in the moderating hypotheses is that high levels of slack and centralization should increase the effect of a political climate on power accumulation (i.e. increase power accumulation from low to moderate or high), but this is not happening here.
} 
The respondent was told that the findings of the quantitative analysis suggest that the effect of centralization and slack is just the opposite. He said, "We are a medium-size company that is privately owned by two engineers, and both of them are closely involved in the operations of the company including product development. These owners and other managers in the organization focus on what is best for the company and not on anyone's personal or political interests." Thus, even though decision making is centralized and slack is plentiful, senior managers do not try to accumulate power.

Sub-Model 2: Effect of Power Accumulation on Politicization of Gate Reviews (with Strict and Objective Criteria and Entrepreneurial/innovative Culture as Moderators): Since senior managers try less to acquire power, they don't attempt to influence gate reviews much. In fact, one of the owners is involved in gate reviews and projects are generally reviewed on their merit. This response is consistent with the quantitative results and the hypothesis.

Gate review criteria are strict and objective, but they don't play any role in influencing the politicization of gate reviews because senior managers try less to politicize the gate reviews. Similarly, even though the culture is entrepreneurial/innovative, it doesn't play a role in the politicization of reviews. These responses are consistent with the quantitative findings but not with the moderating hypotheses.

Sub-Model 3: Effect of Politicization of Gate Reviews on Demotivation of Managers (with Conditionality as a Moderator): Since the level of the politicization of gate reviews is low, it doesn't demotivate product development managers that much. The conditionality of the criteria is low and it doesn't have any role in reducing demotivation because demotivation is low anyway. These responses support both the quantitative findings and the hypotheses.

However, the respondent added, "At times some managers do feel demotivated, but it is not a result of politics. These are mostly technical managers who don't like the criticism of their technical work during project reviews."

\section{Sub-Model 4: Effect of Demotivation on Innovativeness (with Technological and Market}

Turbulence as Moderators): This respondent said that demotivation among product development managers is usually low and it doesn't adversely affect innovativeness. However, he added that if managers are demotivated for some reason, their demotivation adversely impacts innovativeness. This response is consistent with both the hypothesis and the quantitative findings.

"Technological volatility," the respondent said, "is a double-edged sword. If technology changes earlier in the development process, it can be seen as an opportunity to develop cutting edge products. But, if technology changes when the project is almost complete, many parts of the product need to be redesigned. These changes can frustrate product developers and can have an adverse effect on innovativeness. But more often than not, technological change is beneficial." This response is not consistent with the hypothesis and the quantitative findings.

Similarly, market volatility including changes in customer needs can seem detrimental in the short run, but can be beneficial in the long run because it leads to more innovative products that 
better satisfy customers. The overall effect of market volatility is beneficial. This response is consistent with the quantitative findings but not with the moderating hypothesis.

Sub-Model 5: The Effect of Innovativeness on Performance: The respondent said that innovativeness positively affects the market performance of their products. This is consistent with both the hypothesis and the quantitative findings.

\section{New Insights}

This interview suggests that an important determinant of organizational political climate can be the ownership structure of the organization and the direct involvement of the owners in product development and reviews. Future project level research can consider this factor as one of the antecedents of organizational politics.

The second insight suggests that technological volatility can be a double-edged sword. If new technologies emerge earlier in the development process, they can provide opportunities to product designers to develop cutting edge products. However, if technology changes appear much later in the development process when the project is almost complete, many parts of the product need to be redesigned. Such late changes often frustrate product development managers and can have an adverse effect on innovativeness. This issue also needs more attention in future project level studies. 


\section{Appendix 5j}

\section{ELECTCO - Interview Summary}

Respondent's title:

Product:

Number of employees:

Annual sales:

Rank in the industry:

Innovation emphasis:

Entrepreneurial focus:

Technology turbulence:

Market Turbulence:

Centralization:

Slack:

Political Org. Climate:

Number of gates/reviews:

Strictness of the criteria:

Objectivity of the criteria:

Conditionality of the criteria:
Development Lead

Electronic Hardware and Software

About 15,000

About $\$ 2$ billion

Among the top players

7 (on a 1 to 7 scale - where $1=$ not at all and $7=$ a great deal)

7 (on a 1 to 7 scale)

7 (on a 1 to 7 scale)

4 (on a 1 to 7 scale)

3 (on a 1 to 7 scale)

6 (on a 1 to 7 scale)

3 (on a 1 to 7 scale)

4

Moderate

Moderate

Moderate

\section{Feedback on the Model and Unconfirmed Effects}

Sub-Model 1: Effect of Political Climate on Power Accumulation (with Centralization and Slack as Moderators): The climate in the research and development division of this organization is less political and the tendency on the part of senior managers to accumulate power is low. Centralization is low and it doesn't influence power accumulation by senior managers. These responses are consistent with both the quantitative results and the hypotheses.

Slack is high, but this too doesn't affect the level of power accumulation. This response is inconsistent with both the quantitative findings and the moderating hypothesis.

The respondent was told that the findings of quantitative analysis show that slack leads to more power acquisition in a political organizational climate. He said, "The atmosphere in my division is not political to start with. Past and the current chiefs have made a special effort to keep politics away from the product development process. They remain actively involved in product 
development to prevent any external influence from coming into product reviews." So slack doesn't seem to make any difference.

Sub-Model 2: Effect of Power Accumulation on Politicization of Gate Reviews (with Strict and Objective Criteria and Entrepreneurial/Innovative Culture as Moderators): Since power accumulation is low, there is less attempt to politicize gate reviews. This response is consistent with both the quantitative results and the hypothesis.

Review criteria are moderately strict and objective and they don't play a role in influencing the politicization of gate reviews in this environment of low power accumulation. Similarly, the culture is highly entrepreneurial/innovative, but it doesn't affect the politicization of gate reviews. These responses are consistent with the quantitative findings but not with the moderating hypotheses.

The respondent was asked why there are no serious attempts by senior managers to influence gate reviews to get their favourite projects through. He said, "The division develops products in response to customer requirements. So the product specifications and the development schedule are determined as per customer needs. This leaves little scope for senior managers to ask for changes in the project and reduces the opportunity to play politics."

\section{Sub-Model 3: Effect of Politicization of Gate Reviews on Demotivation of Managers (with} Conditionality as a Moderator): The level of demotivation is low in the absence of politicization of gate reviews. This response is consistent with the quantitative findings and with the hypothesis.

A moderate level of conditionality doesn't play any role in influencing demotivation in this set up where politicization of the reviews is already low, which is consistent with the quantitative results but not with the moderator hypothesis.

However, the respondent said, "Demotivation among product development teams comes from other sources. If the project doesn't involve the latest technology as compared to other projects under development, it makes team members less enthusiastic. But, such demotivation does not last long. When the customer appreciates the work done on the project, it usually motivates the members once again."

\section{Sub-Model 4: Effect of Demotivation on Innovativeness (with Technological and Market} Turbulence as Moderators): Since demotivation is generally low, it doesn't have much adverse effect on innovativeness. This response is consistent with the hypothesis and quantitative results.

Technological changes are usually viewed as challenging and interesting and thus energize product development managers and help spur innovativeness. This response contradicts both the quantitative findings and the moderator hypothesis.

This respondent was told that the results suggest that technological volatility reduces innovativeness. He said, "If changes in technology are rapid, they do frustrate product development team members and thus adversely affect their ability to innovate, but this happens 
less often. Since in our organization, teams are less demotivated, they are less frustrated by technological volatility."

Regarding market changes, the respondent said "On a lot of occasions these changes occur because customers request changes in the product as the development is proceeding. Sometimes these requests frustrate managers. But more often, they are seen as opportunities to develop products that better satisfy customers and novel ways are found to achieve this end." Since the product development managers in his organization usually have a low level of demotivation, they can effectively respond to such requests for changes pretty quickly. This explanation seems to be consistent with the results of quantitative analysis but contradicts the moderating hypothesis.

Sub-Model 5: The Effect of Innovativeness on Performance: The respondent said that innovativeness positively affects the performance of new products. This is consistent with both the hypothesis and the quantitative findings.

\section{New Insights}

An insight from this interview is that when, in business-to-business settings, products are developed in response to customer requirements it reduces the opportunity for senior managers to play politics. After all, the product specifications and the development schedule are set as per customer needs and cannot be easily changed, and the project cannot be easily cancelled simply because a senior manager would like to do so to promote his/her interests. Future studies on innovation and politics may want to take this factor into account.

This interview also provided an explanation for the positive moderating effect of market turbulence. If product development teams are not demotivated, they are more likely to see rapid market changes as an opportunity to develop products that better satisfy customers and may even find novel ways to do so. Thus, market turbulence can lead to more innovative outcomes. However, this explanation may not hold when teams are demotivated. 


\section{Appendix 5k}

\section{APPCO - Interview Summary}

Respondent's title:

Product:

Number of employees:

Annual sales:

Rank in the industry:

Innovation emphasis:

Entrepreneurial focus:

Technology turbulence:

Market Turbulence:

Centralization:

Slack:

Political Org. Climate:

Number of gates/reviews:

Strictness of the criteria:

Objectivity of the criteria:

Conditionality of the criteria: Moderate

4

Low

Low
Project Manager

Sports Apparel

About 150

About $\$ 90$ million

Among the top players in the industry

7 (on a 1 to 7 scale - where $1=$ not at all and $7=$ a great deal)

7 (on a 1 to 7 scale)

6 (on a 1 to 7 scale)

7 (on a 1 to 7 scale)

3 (on a 1 to 7 scale)

6 (on a 1 to 7 scale)

3 (on a 1 to 7 scale)

\section{Feedback on the Model and Unconfirmed Effects}

Sub-Model 1: Effect of Political Climate on Power Accumulation (with Centralization and Slack as Moderators): The respondent said that the climate in the organization is less political and as a result power accumulation is low. Centralization is low and it doesn't play any role in enhancing power accumulation in this political climate. These findings are consistent with the hypothesized main effect of political climate and the moderating effect of centralization as well as the quantitative results.

When asked to explain why they have a low level of politics, the respondent said that her organization only develops new products and markets them, but all their manufacturing is outsourced. The organization has merely 150 employees including the managers. The management team is close knit and there is a high degree of connectedness across departments and across management levels and everyone is focused on excelling in the market. This leaves little scope for politics to emerge. 
Slack is high, but it doesn't play a role in influencing power accumulation in this organization with a low level of politics. This response contradicts both the quantitative findings and the moderating hypothesis.

When told that the results of the quantitative analysis suggest that high slack makes senior managers gather more power, she said that since in her organization the level of politics is rather low, the basic drive to acquire surplus resources and become powerful is missing on the part of senior managers. It is entirely possible, she felt, that in a more political organization, slack would lead to higher power accumulation.

Sub-Model 2: Effect of Power Accumulation on Politicization of Gate Reviews (with Strict and Objective Criteria and Entrepreneurial/Innovative Culture as Moderators): In this organization with low power accumulation, senior managers try less to influence the project approval process and thus gate reviews are less politicized. The response is consistent with both the quantitative results and the hypothesis.

Gate review criteria are not so strict or objective and they don't play any role in influencing the politicization of gate reviews. The respondent said, "Since senior managers don't try much to influence gate reviews, there is less intention to use lax review criteria to influence gate review outcomes in their favour." Again, the response is consistent with the quantitative results and supports the moderating hypothesis.

The culture is entrepreneurial and innovative, but doesn't influence the politicization of gate reviews. In essence, the response supports the findings of the quantitative analysis but not the hypothesis.

Sub-Model 3: Effect of Politicization of Gate Reviews on Demotivation of Managers (with Conditionality as a Moderator): Since gate reviews are not politicized, there is not much demotivation among product development managers. This response is consistent with the quantitative findings and the hypothesis.

Also, because gate reviews are less political and demotivation is low, a moderate level of conditionality doesn't play a role in reducing demotivation. In effect, these responses are consistent with the quantitative results but do not support the hypothesis.

Sub-Model 4: Effect of Demotivation on Innovativeness (with Technological and Market Turbulence as Moderators): The respondent said that since demotivation is low, it leads to the development of innovative new products. This response is consistent with the hypothesis and the quantitative findings.

Technologies for this organization are turbulent, but contrary to the quantitative findings and the hypothesis, they don't adversely influence innovativeness. When asked to explain this inconsistency, the respondent said, "Our organization is in fact the source of technological volatility because we are the ones who introduce the latest, cutting edge technologies in our products. Our own induced technological turbulence favors us in developing more innovative products than others in the industry." 
Market turbulence is high and it can help innovativeness. She said, "Our product development managers are not so demotivated and often use market turbulence to identify new opportunities and develop innovative new products." This is consistent with the quantitative findings but not with the hypothesis.

Sub-Model 5: The Effect of Innovativeness on Performance: The organization's innovative products lead to superior market performance, thus supporting the hypothesis and quantitative results.

\section{New Insights}

This interview identifies an antecedent that makes organizational climate less political. This antecedent is a close knit organization that is highly connected across departments and vertically across management levels. By lowering the level of politics, this factor also reduces the extent of power accumulation and the politicization of gate reviews. This dissertation didn't focus on the antecedents of a political climate, but this insight opens up an interesting area for future research.

Organizations differ in terms of whether they use the latest technology or not, and this can influence how technological changes affect new product development. When an organization is at the cutting edge of technology and introduces turbulence in the market through its own product innovations, technology turbulence does not adversely affect the organization. In fact, technology turbulence can play a positive role. 


\section{Appendix 5l}

\section{HARSOCO - Interview Summary}

Respondent's title:

Product:

Number of employees:

Annual sales:

Rank in the industry:

Innovation emphasis:

Entrepreneurial focus:

Technology turbulence:

Market Turbulence:

Centralization:

Slack:

Political Org. Climate:

Number of gates/reviews:

Strictness of the criteria:

Objectivity of the criteria:

Conditionality of the criteria: Low

3

Low

Low
Leader Product Development Group

Electronic hardware and software

About 15,000

About $\$ 7$ billion

Among the top few players in the industry

7 (on a 1 to 7 scale - where $1=$ not at all and $7=$ a great deal)

7 (on a 1 to 7 scale)

7 (on a 1 to 7 scale)

7 (on a 1 to 7 scale)

3 (on a 1 to 7 scale)

3 (on a 1 to 7 scale)

3 (on a 1 to 7 scale)

\section{Feedback on the Model and Unconfirmed Effects}

Sub-Model 1: Effect of Political Climate on Power Accumulation (with Centralization and Slack as Moderators): The respondent said that the climate in the organization is not so political and senior managers don't try much to accumulate power. Centralization is low and doesn't play any role in influencing power accumulation in this climate of low politics. Slack is also low and doesn't influence power accumulation. These responses are consistent with the hypotheses and the results of the quantitative analysis.

The respondent was asked why politics and power accumulation are low in this organization. He said "We have been under severe threat, sales have fallen, and it seemed at one point that we might have to close down. Thousands of employees have been laid off and so for those that have remained it is a question of survival. Everyone knows that this is not the time to play politics; instead, we need to pull together and do our best so that the firm can come out of the problems it has been facing and become a strong player in the industry again." In effect, this threat to survival reduces the extent of politics and through it, power accumulation. 
Sub-Model 2: Effect of Power Accumulation on Politicization of Gate Reviews (with Strict and Objective Criteria and Entrepreneurial/Innovative Culture as Moderators): Since managers are not trying much to accumulate power, gate reviews are not so politicized. Gate review criteria have low levels of strictness and objectivity and don't influence the politicization of gate reviews. The respondent said, "Since senior managers don't attempt to influence gate reviews, they don't feel the need to exploit the less rigorous criteria to influence gate review outcomes in their favour." These responses are consistent with both the quantitative findings and hypotheses.

The organization has a high entrepreneurial and innovative focus, but this doesn't affect politicization. The above response is consistent with the quantitative results but contradicts the hypothesis.

Sub-Model 3: Effect of Politicization of Gate Reviews on Demotivation of Managers (with Conditionality as a Moderator): As the gate reviews are not politicized, product development managers' demotivation is low. This response is consistent with both the results of the quantitative findings and the hypothesis.

Also, conditionality is low and it doesn't play a role in reducing demotivation because as such gate reviews are less political and demotivation is low. Thus, these responses support the hypothesis and quantitative results.

\section{Sub-Model 4: Effect of Demotivation on Innovativeness (with Technological and Market} Turbulence as Moderators): Since product development managers are less demotivated, innovativeness of new products developed by them is high. However, the respondent said that demotivation, no doubt, can lower innovativeness of new products. The response supports the hypothesis and the quantitative results.

The organization faces turbulent markets and technology. Both of these factors play a positive role, because managers view turbulence in markets and technology as opportunities and develop more innovative products. This seems to happen because the organization faces a threat to its survival and as a consequence, product development managers are motivated to exploit every opportunity to innovate. The above response supports the quantitative findings about the effect of market turbulence but not the moderating hypothesis. On the other hand, the response contradicts both the results and hypothesis about the effect of technological turbulence.

Sub-Model 5: The Effect of Innovativeness on Performance: The respondent said that innovative new products in turn lead to better market performance. This is consistent with both the hypothesis and the quantitative findings.

\section{New Insights}

An important learning from this interview is that a threat to the organization's survival can be a potent force that can make the organizational climate less political and through it reduce senior managers' tendency to accumulate power. It is possible though that such a threat can lead some organizations to give up and become fatalistic. However, if the organizational leadership is able 
to motivate the organization to respond with a positive attitude and purposeful action, it can lead to organizational renewal. Future studies can examine the role of threat to survival as an antecedent of organizational political climate.

Another major insight is that turbulence can be viewed as either an opportunity or a threat by product development managers. If it is seen as an opportunity, as it is in this organization, it can give a positive impetus to innovation. On the other hand, if it is seen as a threat, it can worsen the demotivation-innovativeness relationship. 
Appendix 6: Summary of Organizations and Respondents in Qualitative Research

\begin{tabular}{|c|c|c|c|c|c|c|c|c|c|c|c|c|}
\hline $\begin{array}{l}\text { Org/ } \\
\text { Division" }\end{array}$ & Compco & Machco & Filtco & Elecmaco & Fabrico & Softco & Semco & Comapco & Indautco & Electco & Appco & Harsoco \\
\hline $\begin{array}{l}\text { Appendix } \\
\text { (Detalled } \\
\text { Summary) }\end{array}$ & $5 \mathbf{a}$ & $\mathbf{5 b}$ & 5c & $5 d$ & $5 e$ & $5 f$ & $5 g$ & $\mathbf{5 h}$ & $5 \mathbf{i}$ & $\mathbf{5 j}$ & $5 \mathbf{k}$ & 5l \\
\hline $\begin{array}{l}\text { Mangger } \\
\text { Title }\end{array}$ & $\begin{array}{l}\text { Product } \\
\text { Team } \\
\text { Leader }\end{array}$ & $\begin{array}{l}\text { NPD. } \\
\text { Leader }\end{array}$ & $\begin{array}{l}\text { Project } \\
\text { Mgr. }\end{array}$ & $\begin{array}{l}\text { Project } \\
\text { Leader }\end{array}$ & $\begin{array}{l}\text { Project } \\
\text { Mgr. }\end{array}$ & $\begin{array}{l}\text { Director } \\
\text { NPD. }\end{array}$ & $\begin{array}{l}\text { Senior } \\
\text { Proj. } \\
\text { Engineer }\end{array}$ & $\begin{array}{l}\text { Senior } \\
\text { NPD. } \\
\text { Eng. }\end{array}$ & $\begin{array}{l}\text { Project } \\
\text { Manager }\end{array}$ & $\begin{array}{l}\text { Develop. } \\
\text { Lead }\end{array}$ & $\begin{array}{l}\text { Project } \\
\text { Mgr. }\end{array}$ & $\begin{array}{l}\text { Leader } \\
\text { Product } \\
\text { Develop. }\end{array}$ \\
\hline Product & $\begin{array}{l}\text { Compo. } \\
\text { for auto } \\
\text { industry }\end{array}$ & $\begin{array}{l}\text { Precision } \\
\text { machine } \\
\text { tools }\end{array}$ & $\begin{array}{l}\text { Water } \\
\text { filtration } \\
\text { systems }\end{array}$ & $\begin{array}{l}\text { Electronic } \\
\text { machines }\end{array}$ & $\begin{array}{l}\text { Fabricated } \\
\text { assemblies }\end{array}$ & Software & $\begin{array}{l}\text { Semi- } \\
\text { conductor }\end{array}$ & $\begin{array}{l}\text { Appliance } \\
\text { controls }\end{array}$ & $\begin{array}{l}\text { Industrial } \\
\text { automation }\end{array}$ & $\begin{array}{l}\text { Electronic } \\
\text { hardware, } \\
\text { software }\end{array}$ & $\begin{array}{l}\text { Sports } \\
\text { apparel }\end{array}$ & $\begin{array}{l}\text { Electronic } \\
\text { hardware . } \\
\text { software }\end{array}$ \\
\hline $\begin{array}{l}\text { Enterprise } \\
\text { Size }\end{array}$ & Large & Large & Large & Large & Large & Med. & Large & Med. & Med. & Large & Med. & Large \\
\hline Employees & 30,000 & 700 & 10,000 & 18,000 & 3,000 & 120 & 20,000 & 350 & 150 & 15,000 & 150 & 15,000 \\
\hline Sales (\$m.) & 6,000 & 100 & 3,000 & 10,000 & $\begin{array}{l}\text { Not } \\
\text { provided }\end{array}$ & 20 & 8,000 & 100 & 40 & 2,000 & 90 & 7,000 \\
\hline $\begin{array}{l}\text { Number of } \\
\text { Gates }\end{array}$ & 6 & 4 & 5 & 5 & 5 & 3 & 3 & 3 & 5 & 4 & 4 & 3 \\
\hline $\begin{array}{l}\text { Political } \\
\text { Org. } \\
\text { Climate }^{c}\end{array}$ & 6 & 6 & 6 & 6 & 6 & 6 & 4 & 4 & 3 & 3 & 3 & 3 \\
\hline
\end{tabular}

${ }^{b}$ All the respondents belonged to the new product development or R\&D department.

c On a 1 to 7 scale where $1=$ Not at all and $7=A$ Great Deal. 
Appendix 7: Qualitative Responses for Sub-Model 1: Effect of Political Organization Climate on Power Accumulation with Moderation by Centralization and Slack

\begin{tabular}{|c|c|c|c|c|c|c|c|c|c|c|c|c|}
\hline $\begin{array}{l}\text { ORG/DIVISION } \\
\text { (APPENDIX) }\end{array}$ & $\begin{array}{l}\text { Compco } \\
5 a\end{array}$ & $\begin{array}{l}\text { Machco } \\
5 b\end{array}$ & $\begin{array}{l}\text { Filtco } \\
5 \mathrm{c}\end{array}$ & $\begin{array}{l}\text { Elecmaco } \\
5 \mathrm{~d}\end{array}$ & $\begin{array}{l}\text { Fabrico } \\
5 \mathrm{e}\end{array}$ & $\begin{array}{l}\text { Softco } \\
5 f\end{array}$ & $\begin{array}{l}\text { Semco } \\
5 \mathrm{~g}\end{array}$ & $\begin{array}{l}\text { Comapco } \\
5 \mathrm{~h}\end{array}$ & $\begin{array}{l}\text { Indautco } \\
5 i\end{array}$ & $\begin{array}{l}\text { Electco } \\
5 j\end{array}$ & $\begin{array}{l}\text { Appco } \\
5 k\end{array}$ & $\begin{array}{l}\text { Harsoco } \\
51\end{array}$ \\
\hline $\begin{array}{l}\text { Political Org. } \\
\text { Climate }^{c}\end{array}$ & $\mathrm{Hi}(6)$ & $\mathrm{Hi} \mathrm{(6)}$ & $\mathrm{Hi}(6)$ & $\mathrm{Hi}(6)$ & $\mathrm{Hi}(6)$ & $\mathrm{Hi}(6)$ & Mod (4) & Mod (4) & Lo(3) & Lo (3) & Lo(3) & Lo (3) \\
\hline $\begin{array}{l}\text { Power } \\
\text { Accumulation }\end{array}$ & $\mathrm{Hi}$ & $\mathrm{Hi}$ & $\mathrm{Hi}$ & $\mathrm{Hi}$ & $\mathrm{Hi}$ & $\mathrm{Hi}$ & Mod & Mod & Lo & Lo & Lo & Lo \\
\hline $\begin{array}{l}\frac{\text { Political Climate - }}{\text { Power Accum. }} \\
\frac{\text { Consistent }^{2}}{\text { Supported }^{\mathrm{c}}}\end{array}$ & $\begin{array}{l}\text { Yes } \\
\text { Yes }\end{array}$ & $\begin{array}{l}\text { Yes } \\
\text { Yes }\end{array}$ & $\begin{array}{l}\text { Yes } \\
\text { Yes }\end{array}$ & $\begin{array}{l}\text { Yes } \\
\text { Yes }\end{array}$ & $\begin{array}{l}\text { Yes } \\
\text { Yes }\end{array}$ & $\begin{array}{l}\text { Yes } \\
\text { Yes }\end{array}$ & $\begin{array}{l}\text { Yes } \\
\text { Yes }\end{array}$ & $\begin{array}{l}\text { Yes } \\
\text { Yes }\end{array}$ & $\begin{array}{l}\text { Yes } \\
\text { Yes }\end{array}$ & $\begin{array}{l}\text { Yes } \\
\text { Yes }\end{array}$ & $\begin{array}{l}\text { Yes } \\
\text { Yes }\end{array}$ & $\begin{array}{l}\text { Yes } \\
\text { Yes }\end{array}$ \\
\hline Centralization $^{\mathrm{C}}$ & $\operatorname{Mod}(4)$ & $\mathrm{Hi} \mathrm{(6)}$ & Hi (5) & $\mathrm{Hi}(6)$ & $\mathrm{Hi}(5)$ & $\mathrm{Hi}(7)$ & Lo (3) & Lo (3) & $\mathrm{Hi}(5)$ & Lo (3) & Lo (3) & Lo (3) \\
\hline $\begin{array}{l}\text { MOD.: Centraliz. } \\
\text { Consistent }^{a} \\
\text { Supported }^{b}\end{array}$ & $\begin{array}{l}\text { Yes } \\
\text { Yes }\end{array}$ & $\begin{array}{l}\text { Yes } \\
\text { Yes }\end{array}$ & $\begin{array}{l}\text { Yes } \\
\text { Yes }\end{array}$ & $\begin{array}{l}\text { Yes } \\
\text { Yes }\end{array}$ & $\begin{array}{l}\text { Yes } \\
\text { Yes }\end{array}$ & $\begin{array}{l}\text { Yes } \\
\text { Yes }\end{array}$ & $\begin{array}{l}\text { Yes } \\
\text { Yes }\end{array}$ & $\begin{array}{l}\text { Yes } \\
\text { Yes }\end{array}$ & $\begin{array}{l}\text { No } \\
\text { No }\end{array}$ & $\begin{array}{l}\text { Yes } \\
\text { Yes }\end{array}$ & $\begin{array}{l}\text { Yes } \\
\text { Yes }\end{array}$ & $\begin{array}{l}\text { Yes } \\
\text { Yes }\end{array}$ \\
\hline Slack $^{c}$ & Lo (3) & $\mathrm{Hi}(6)$ & Lo (3) & $\mathrm{Hi}(5)$ & Lo (3) & $\mathrm{Hi}(6)$ & Lo (2) & Lo (3) & $\mathrm{Hi} \mathrm{(7)}$ & Hi (6) & $\mathrm{Hi}(6)$ & Lo (3) \\
\hline $\begin{array}{l}\text { MOD.: Slack } \\
\text { Consistent" }^{\prime \prime} \\
\text { Supported }^{b}\end{array}$ & $\begin{array}{l}\text { Yes } \\
\text { Yes }\end{array}$ & $\begin{array}{l}\text { Yes } \\
\text { Yes }\end{array}$ & $\begin{array}{l}\text { Yes } \\
\text { Yes }\end{array}$ & $\begin{array}{l}\text { No } \\
\text { No }\end{array}$ & $\begin{array}{l}\text { Yes } \\
\text { Yes }\end{array}$ & $\begin{array}{l}\text { Yes } \\
\text { Yes }\end{array}$ & $\begin{array}{l}\text { Yes } \\
\text { Yes }\end{array}$ & $\begin{array}{l}\text { Yes } \\
\text { Yes }\end{array}$ & $\begin{array}{l}\text { No } \\
\text { No }\end{array}$ & $\begin{array}{l}\text { No } \\
\text { No }\end{array}$ & $\begin{array}{l}\text { No } \\
\text { No }\end{array}$ & $\begin{array}{l}\text { Yes } \\
\text { Yes }\end{array}$ \\
\hline
\end{tabular}

Consistent with the quantitative findings.

${ }^{b}$ Hypothesis supported.

${ }^{c}$ Numbers in parentheses are on a 1 to 7 scale, where $1=$ Not at all and $7=$ A Great Deal. 
Appendix 8: Qualitative Responses for Sub-Model 2: Effect of Power Accumulation on Politicization of Gate Reviews with Moderation by Strict and Objective Criteria and Entrepreneurial/Innovative Culture

\begin{tabular}{|c|c|c|c|c|c|c|c|c|c|c|c|c|}
\hline $\begin{array}{l}\text { ORG/DIVISION } \\
\text { (APPENDIX) }\end{array}$ & $\begin{array}{l}\text { Compco } \\
5 a\end{array}$ & $\begin{array}{l}\text { Machco } \\
5 b\end{array}$ & $\begin{array}{l}\text { Filtco } \\
5 c\end{array}$ & $\begin{array}{l}\text { Elecmaco } \\
5 d\end{array}$ & $\begin{array}{l}\text { Fabrico } \\
5 e\end{array}$ & $\begin{array}{l}\text { Softco } \\
5 f\end{array}$ & $\begin{array}{l}\text { Semco } \\
5 g\end{array}$ & $\begin{array}{l}\text { Comapco } \\
5 \mathrm{~h}\end{array}$ & $\begin{array}{l}\text { Indautco } \\
5 i\end{array}$ & $\begin{array}{l}\text { Electco } \\
5]\end{array}$ & $\begin{array}{l}\text { Appco } \\
5 k\end{array}$ & $\begin{array}{l}\text { Harsoco } \\
51\end{array}$ \\
\hline Power Accum & $\mathrm{Hi}$ & $\mathrm{Hi}$ & $\mathrm{Hi}$ & $\mathrm{Hi}$ & $\mathrm{Hi}$ & $\mathrm{Hi}$ & Mod & Mod & Lo & Lo & Lo & Lo \\
\hline Politicization & $\mathrm{Hi}$ & $\mathrm{Hi}$ & $\mathrm{Hi}$ & $\mathrm{Hi}$ & $\mathrm{Hi}$ & Mod & Mod & Lo & Lo & Lo & Lo & Lo \\
\hline $\begin{array}{l}\text { Power Accum } \\
\frac{- \text { Politiciz. }}{\text { Consistent }^{\mathrm{a}}} \\
\text { Supported }^{\mathrm{b}}\end{array}$ & $\begin{array}{l}\text { Yes } \\
\text { Yes }\end{array}$ & $\begin{array}{l}\text { Yes } \\
\text { Yes }\end{array}$ & $\begin{array}{l}\text { Yes } \\
\text { Yes }\end{array}$ & $\begin{array}{l}\text { Yes } \\
\text { Yes }\end{array}$ & $\begin{array}{l}\text { Yes } \\
\text { Yes }\end{array}$ & $\begin{array}{l}\text { Yes } \\
\text { Yes }\end{array}$ & $\begin{array}{l}\text { Yes } \\
\text { Yes }\end{array}$ & $\begin{array}{l}\text { No } \\
\text { No }\end{array}$ & $\begin{array}{l}\text { Yes } \\
\text { Yes }\end{array}$ & $\begin{array}{l}\text { Yes } \\
\text { Yes }\end{array}$ & $\begin{array}{l}\text { Yes } \\
\text { Yes }\end{array}$ & $\begin{array}{l}\text { Yes } \\
\text { Yes }\end{array}$ \\
\hline Strict/Obj Cri & Mod/Mod & Lo/Lo & Lo/Lo & Lo/Lo & Lo/Lo & $\mathrm{Hi} / \mathrm{Hi}$ & Lo/Lo & $\mathrm{Hi} / \mathrm{Hi}$ & $\mathrm{Hi} / \mathrm{Hi}$ & Mod/Mod & Lo/Lo & Lo/Lo \\
\hline $\begin{array}{l}\text { MOD. : Strict } \\
\frac{\text { \& Obi. Cri. }}{\text { Consistent }^{\star 2}} \\
\text { Supported }^{b}\end{array}$ & $\begin{array}{l}\text { Yes } \\
\text { No }\end{array}$ & $\begin{array}{l}\text { Yes } \\
\text { Yes }\end{array}$ & $\begin{array}{l}\text { Yes } \\
\text { Yes }\end{array}$ & $\begin{array}{l}\text { Yes } \\
\text { Yes }\end{array}$ & $\begin{array}{l}\text { Yes } \\
\text { Yes }\end{array}$ & $\begin{array}{l}\text { Yes } \\
\text { No }\end{array}$ & $\begin{array}{l}\text { Yes } \\
\text { Yes }\end{array}$ & $\begin{array}{l}\text { Yes } \\
\text { No }\end{array}$ & $\begin{array}{l}\text { Yes } \\
\text { No }\end{array}$ & $\begin{array}{l}\text { Yes } \\
\text { No }\end{array}$ & $\begin{array}{l}\text { Yes } \\
\text { Yes }\end{array}$ & $\begin{array}{l}\text { Yes } \\
\text { Yes }\end{array}$ \\
\hline Ent/Inn Cult & $\mathrm{Hi}(5 / 5)$ & Lo $(2 / 3)$ & Lo $(3 / 3)$ & $\mathrm{Hi}(7 / 6)$ & Lo $(3 / 3)$ & $\mathrm{Hi}(7 / 7)$ & $\mathrm{Hi}(6 / 5)$ & Lo $(3 / 3)$ & $\mathrm{Hi}(7 / 7)$ & $\mathrm{Hi}(7 / 7)$ & $\mathrm{Hi}(7 / 7)$ & $\mathrm{Hi}(7 / 7)$ \\
\hline $\begin{array}{l}\text { MOD.: Inn } \\
\frac{\text { Cult. }}{\text { Consistent }^{\mathrm{a}}} \\
\text { Supported }^{\mathrm{b}}\end{array}$ & $\begin{array}{l}\text { Yes } \\
\text { No }\end{array}$ & $\begin{array}{l}\text { Yes } \\
\text { Yes }\end{array}$ & $\begin{array}{l}\text { Yes } \\
\text { Yes }\end{array}$ & $\begin{array}{l}\text { Yes } \\
\text { No }\end{array}$ & $\begin{array}{l}\text { Yes } \\
\text { Yes }\end{array}$ & $\begin{array}{l}\text { No } \\
\text { Yes }\end{array}$ & $\begin{array}{l}\text { Yes } \\
\text { No }\end{array}$ & $\begin{array}{l}\text { Yes } \\
\text { Yes }\end{array}$ & $\begin{array}{l}\text { Yes } \\
\text { No }\end{array}$ & $\begin{array}{l}\text { Yes } \\
\text { No }\end{array}$ & $\begin{array}{l}\text { Yes } \\
\text { No }\end{array}$ & $\begin{array}{l}\text { Yes } \\
\text { No }\end{array}$ \\
\hline
\end{tabular}

"Consistent with the quantitative findings.

bHypothesis supported.

'Numbers in parentheses are on a 1 to 7 scale, where $1=$ Not at all and $7=A$ Great Deal. The two numbers refer to the innovation and entrepreneurial foci respectively 
Appendix 9: Qualitative Responses for Sub-Model 3: Effect of Politicization of Gate Reviews on Demotivation with Moderation by Conditional Criteria

\begin{tabular}{|c|c|c|c|c|c|c|c|c|c|c|c|c|}
\hline $\begin{array}{l}\text { ORG/DIVISION } \\
\text { (APPENDIX) }\end{array}$ & $\begin{array}{l}\text { Compco } \\
5 a\end{array}$ & $\begin{array}{l}\text { Machco } \\
5 b\end{array}$ & Filtco $5 c$ & $\begin{array}{l}\text { Elecmaco } \\
5 \mathrm{~d}\end{array}$ & $\begin{array}{l}\text { Fabrico } \\
5 e\end{array}$ & $\begin{array}{l}\text { Softco } \\
5 f\end{array}$ & $\begin{array}{l}\text { Semco } \\
5 g\end{array}$ & $\begin{array}{l}\text { Comappco } \\
5 \mathrm{~h}\end{array}$ & $\begin{array}{l}\text { Indautco } \\
5 i\end{array}$ & $\begin{array}{l}\text { Electco } \\
5 j\end{array}$ & $\begin{array}{l}\text { Appco } \\
5 k\end{array}$ & $\begin{array}{l}\text { Harsoco } \\
51\end{array}$ \\
\hline Politicization & $\mathrm{Hi}$ & $\mathrm{Hi}$ & $\mathrm{Hi}$ & $\mathrm{Hi}$ & $\mathrm{Hi}$ & Mod & Mod & Lo & Lo & Lo & Lo & Lo \\
\hline Demotivation & $\mathrm{Hi}$ & $\mathrm{Hi}$ & $\mathrm{Hi}$ & $\mathrm{Hi}$ & $\mathrm{Hi}$ & Mod & Mod & Lo & Lo & Lo & Lo & Lo \\
\hline $\begin{array}{l}\frac{\text { Politicization - }}{\text { Demotivation }} \\
\text { Consistent" }^{\text {" }} \\
\text { Supported }^{\text {b }}\end{array}$ & $\begin{array}{l}\text { Yes } \\
\text { Yes }\end{array}$ & $\begin{array}{l}\text { Yes } \\
\text { Yes }\end{array}$ & $\begin{array}{l}\text { Yes } \\
\text { Yes }\end{array}$ & $\begin{array}{l}\text { Yes } \\
\text { Yes }\end{array}$ & $\begin{array}{l}\text { Yes } \\
\text { Yes }\end{array}$ & $\begin{array}{l}\text { Yes } \\
\text { Yes }\end{array}$ & $\begin{array}{l}\text { Yes } \\
\text { Yes }\end{array}$ & $\begin{array}{l}\text { Yes } \\
\text { Yes }\end{array}$ & $\begin{array}{l}\text { Yes } \\
\text { Yes }\end{array}$ & $\begin{array}{l}\text { Yes } \\
\text { Yes }\end{array}$ & $\begin{array}{l}\text { Yes } \\
\text { Yes }\end{array}$ & $\begin{array}{l}\text { Yes } \\
\text { Yes }\end{array}$ \\
\hline $\begin{array}{l}\text { Conditional } \\
\text { Criteria }\end{array}$ & Mod & Mod & $\mathrm{Hi}$ & Lo & Lo & Lo & Mod & Lo & Lo & Mod & Mod & Lo \\
\hline $\begin{array}{l}\text { MOD.: Condi. } \\
\text { Criteria } \\
\text { Consistent }^{2} \\
\text { Supported }^{\mathrm{b}}\end{array}$ & $\begin{array}{l}\text { Yes } \\
\text { No }\end{array}$ & $\begin{array}{l}\text { Yes } \\
\text { No }\end{array}$ & $\begin{array}{l}\text { Yes } \\
\text { No }\end{array}$ & $\begin{array}{l}\text { Yes } \\
\text { Yes }\end{array}$ & $\begin{array}{l}\text { Yes } \\
\text { Yes }\end{array}$ & $\begin{array}{l}\text { Yes } \\
\text { Yes }\end{array}$ & $\begin{array}{l}\text { Yes } \\
\text { No }\end{array}$ & $\begin{array}{l}\text { Yes } \\
\text { Yes }\end{array}$ & $\begin{array}{l}\text { Yes } \\
\text { Yes }\end{array}$ & $\begin{array}{l}\text { Yes } \\
\text { No }\end{array}$ & $\begin{array}{l}\text { Yes } \\
\text { No }\end{array}$ & $\begin{array}{l}\text { Yes } \\
\text { Yes }\end{array}$ \\
\hline
\end{tabular}

" Consistent with the quantitative findings.

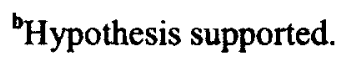


Appendix 10: Qualitative Responses for Sub-Model 4: Effect of Demotivation on Innovativeness with Moderation by Technological and Market Turbulence

\begin{tabular}{|c|c|c|c|c|c|c|c|c|c|c|c|c|}
\hline $\begin{array}{l}\text { ORG/DIVISION } \\
\text { (APPENDIX) }\end{array}$ & $\begin{array}{l}\text { Compco } \\
5 a\end{array}$ & $\begin{array}{l}\text { Machco } \\
\text { 5b }\end{array}$ & $\begin{array}{l}\text { Filtco } \\
5 c\end{array}$ & $\begin{array}{l}\text { Elecmaco } \\
5 \mathrm{~d}\end{array}$ & $\begin{array}{l}\text { Fabrico } \\
5 e\end{array}$ & $\begin{array}{l}\text { Softco } \\
5 f\end{array}$ & $\begin{array}{l}\text { Semco } \\
5 g\end{array}$ & $\begin{array}{l}\text { Comappco } \\
5 \mathrm{~h}\end{array}$ & $\begin{array}{l}\text { Indautco } \\
5 i\end{array}$ & $\begin{array}{l}\text { Electco } \\
5 \mathbf{j}\end{array}$ & $\begin{array}{l}\text { Appco } \\
5 k\end{array}$ & $\begin{array}{l}\text { Harsoco } \\
5 !\end{array}$ \\
\hline Demotivation & $\mathrm{Hi}$ & $\mathrm{Hi}$ & $\mathrm{Hi}$ & $\mathrm{Hi}$ & $\mathrm{Hi}$ & Mod & Mod & Lo & Lo & Lo & Lo & Lo \\
\hline Innovativeness & Lo & Lo & Lo & Lo & Lo & Mod & Mod & $\mathrm{Hi}$ & $\mathrm{Hi}$ & $\mathrm{Hi}$ & $\mathrm{Hi}$ & $\mathrm{Hi}$ \\
\hline $\begin{array}{l}\frac{\text { Demot }-}{\text { Consistent }^{2}} \\
\text { Supported }^{b}\end{array}$ & $\begin{array}{l}\text { Yes } \\
\text { Yes }\end{array}$ & $\begin{array}{l}\text { Yes } \\
\text { Yes }\end{array}$ & $\begin{array}{l}\text { Yes } \\
\text { Yes }\end{array}$ & $\begin{array}{l}\text { Yes } \\
\text { Yes }\end{array}$ & $\begin{array}{l}\text { Yes } \\
\text { Yes }\end{array}$ & $\begin{array}{l}\text { Yes } \\
\text { Yes }\end{array}$ & $\begin{array}{l}\text { Yes } \\
\text { Yes }\end{array}$ & $\begin{array}{l}\text { Yes } \\
\text { Yes }\end{array}$ & $\begin{array}{l}\text { Yes } \\
\text { Yes }\end{array}$ & $\begin{array}{l}\text { Yes } \\
\text { Yes }\end{array}$ & $\begin{array}{l}\text { Yes } \\
\text { Yes }\end{array}$ & $\begin{array}{l}\text { Yes } \\
\text { Yes }\end{array}$ \\
\hline Tech. Turb. & $\mathrm{Hi}(5)$ & Lo (2) & Lo (3) & $\mathrm{Hi}(6)$ & Lo (3) & Hi (7) & $\mathrm{Hi}(7)$ & $\operatorname{Mod}(4)$ & $\mathrm{Hi}(7)$ & $\mathrm{Hi}(7)$ & $\mathrm{Hi} \mathrm{(6)}$ & $\mathrm{Hi} \mathrm{(7)}$ \\
\hline $\begin{array}{l}\text { MOD.: Tech. } \\
\frac{\text { Turbulence }}{\text { Consistent }^{\text {" }}} \\
\text { Supported }^{\text {b }}\end{array}$ & $\begin{array}{l}\text { Yes } \\
\text { Yes }\end{array}$ & $\begin{array}{l}\text { Yes } \\
\text { Yes }\end{array}$ & $\begin{array}{l}\text { Yes } \\
\text { Yes }\end{array}$ & $\begin{array}{l}\text { No } \\
\text { No }\end{array}$ & $\begin{array}{l}\text { Yes } \\
\text { Yes }\end{array}$ & $\begin{array}{l}\text { No } \\
\text { No }\end{array}$ & $\begin{array}{l}\text { Yes } \\
\text { Yes }\end{array}$ & $\begin{array}{l}\text { No } \\
\text { No }\end{array}$ & $\begin{array}{l}\text { No } \\
\text { No }\end{array}$ & $\begin{array}{l}\text { No } \\
\text { No }\end{array}$ & $\begin{array}{l}\text { No } \\
\text { No }\end{array}$ & $\begin{array}{l}\text { No } \\
\text { No }\end{array}$ \\
\hline Market Turb. & $\mathrm{Hi}(5)$ & $\mathrm{Hi}(5)$ & Lo (3) & Lo (3) & Lo (3) & Hi (7) & $\mathrm{Hi}(6)$ & Lo (3) & $\mathrm{Hi}(7)$ & $\operatorname{Mod}(4)$ & $\mathrm{Hi}(7)$ & $\mathrm{Hi}(7)$ \\
\hline $\begin{array}{l}\text { MOD.: } \\
\frac{\text { Market Turb. }}{\text { Consistent" }} \\
\text { Supported }^{\text {b }}\end{array}$ & $\begin{array}{l}\text { No } \\
\text { Yes }\end{array}$ & $\begin{array}{l}\text { No } \\
\text { Yes }\end{array}$ & $\begin{array}{l}\text { Yes } \\
\text { Yes }\end{array}$ & $\begin{array}{l}\text { Yes } \\
\text { Yes }\end{array}$ & $\begin{array}{l}\text { Yes } \\
\text { Yes }\end{array}$ & $\begin{array}{l}\text { Yes } \\
\text { No }\end{array}$ & $\begin{array}{l}\text { No } \\
\text { Yes }\end{array}$ & $\begin{array}{l}\text { Yes } \\
\text { Yes }\end{array}$ & $\begin{array}{l}\text { Yes } \\
\text { No }\end{array}$ & $\begin{array}{l}\text { Yes } \\
\text { No }\end{array}$ & $\begin{array}{l}\text { Yes } \\
\text { No }\end{array}$ & $\begin{array}{l}\text { Yes } \\
\text { No }\end{array}$ \\
\hline
\end{tabular}

" Consistent with the quantitative findings.

${ }^{b}$ Hypothesis supported.

${ }^{\mathrm{c}}$ Numbers in parentheses are on a 1 to 7 scale, where $1=$ Not at all and $7=\mathrm{A}$ Great Deal. 
Appendix 11: Qualitative Responses for Sub-Model 5: Effect of Innovativeness on Performance

\begin{tabular}{|c|c|c|c|c|c|c|c|c|c|c|c|c|}
\hline $\begin{array}{l}\text { ORG/DIVISION } \\
\text { (APPENDIX) }\end{array}$ & $\begin{array}{l}\text { Compco } \\
5 a\end{array}$ & $\begin{array}{l}\text { Machco } \\
5 b\end{array}$ & $\begin{array}{l}\text { Filtco } \\
5 \mathrm{C}\end{array}$ & $\begin{array}{l}\text { Elecmaco } \\
5 d\end{array}$ & $\begin{array}{l}\text { Fabrico } \\
5 e\end{array}$ & $\begin{array}{l}\text { Softco } \\
5 f\end{array}$ & $\begin{array}{l}\text { Semco } \\
5 g\end{array}$ & $\begin{array}{l}\text { Comappco } \\
5 \mathrm{~h}\end{array}$ & $\begin{array}{l}\text { Indautco } \\
5 i\end{array}$ & $\begin{array}{l}\text { Electco } \\
5 \mathbf{j}\end{array}$ & $\begin{array}{l}\text { Appco } \\
5 k\end{array}$ & $\begin{array}{l}\text { Harsoco } \\
51\end{array}$ \\
\hline Innovativeness & Low & Low & Low & Low & Low & Mod & Mod & High & High & High & High & High \\
\hline Performance & Low & Low & Mod & Low & Low & Mod & Mod & High & High & High & High & High \\
\hline $\begin{array}{l}\frac{\text { Innov. }-}{\text { Performance }} \\
\frac{\text { Consistent }}{2} \\
\text { Supported }^{b}\end{array}$ & $\begin{array}{l}\text { Yes } \\
\text { Yes }\end{array}$ & $\begin{array}{l}\text { Yes } \\
\text { Yes }\end{array}$ & $\begin{array}{l}\text { Yes } \\
\text { Yes }\end{array}$ & $\begin{array}{l}\text { Yes } \\
\text { Yes }\end{array}$ & $\begin{array}{l}\text { Yes } \\
\text { Yes }\end{array}$ & $\begin{array}{l}\text { Yes } \\
\text { Yes }\end{array}$ & $\begin{array}{l}\text { Yes } \\
\text { Yes }\end{array}$ & $\begin{array}{l}\text { Yes } \\
\text { Yes }\end{array}$ & $\begin{array}{l}\text { Yes } \\
\text { Yes }\end{array}$ & $\begin{array}{l}\text { Yes } \\
\text { Yes }\end{array}$ & $\begin{array}{l}\text { Yes } \\
\text { Yes }\end{array}$ & $\begin{array}{l}\text { Yes } \\
\text { Yes }\end{array}$ \\
\hline
\end{tabular}

Consistent with the quantitative findings.

${ }^{\mathrm{b}}$ Hypothesis supported 